\title{
A corpus based, lexical analysis of patient information for radiography
}

Richards Golini, Catherine

How to cite:

Richards Golini, Catherine (2019) A corpus based, lexical analysis of patient information for radiography. Doctoral thesis, Swansea University.

http://cronfa.swan.ac.uk/Record/cronfa51643

Use policy:

This item is brought to you by Swansea University. Any person downloading material is agreeing to abide by the terms of the repository licence: copies of full text items may be used or reproduced in any format or medium, without prior permission for personal research or study, educational or non-commercial purposes only. The copyright for any work remains with the original author unless otherwise specified. The full-text must not be sold in any format or medium without the formal permission of the copyright holder. Permission for multiple reproductions should be obtained from the original author.

Authors are personally responsible for adhering to copyright and publisher restrictions when uploading content to the repository.

Please link to the metadata record in the Swansea University repository, Cronfa (link given in the citation reference above.)

http://www.swansea.ac.uk/library/researchsupport/ris-support/ 
A corpus based, lexical analysis of patient information for radiography

Catherine Richards Golini

Submitted to Swansea University in fulfilment of the requirements for the Degree of Doctor of Philosophy

Swansea University

2018 


\section{DECLARATION}

This work has not previously been accepted in substance for any degree and is not being concurrently submitted in candidature for any degree.

Signed

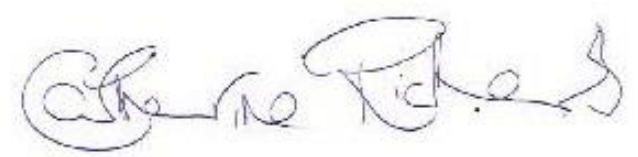

Date 27 November 2018

(candidate)

\section{STATEMENT 1}

This thesis is the result of my own investigations, except where otherwise stated.

Other sources are acknowledged by footnotes giving explicit references. A bibliography is appended.

Signed

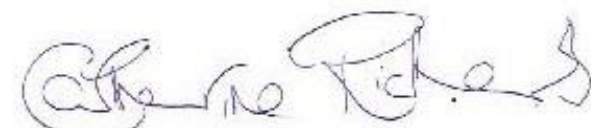

(candidate)

Date 27 November 2018

\section{STATEMENT 2}

I hereby give consent for my thesis, if accepted, to be available for photocopying and for inter-library loan, and for the title and summary to be made available to outside organisations.

Signed

(candidate)

Date 27 November 2018 


\section{Acknowledgements}

I would like to express my gratitude and thanks to my supervisor Cornelia Tschichold, to other staff members in the Applied Linguistics department at Swansea, and all associates of the former Vocabulary Acquisition Research Group who have given me encouragement, support and friendship over the last 7 years.

Special thanks go to my parents for always showing interest, and to my husband, Stefano, for his immense support and super-human levels of patience. 


\begin{abstract}
Despite the importance and the ubiquity of medical patient information in many healthcare systems in the world, we know very little about the lexical characteristics of the register. We do not know how patients perceive the information in the leaflets or whether the messages are transmitted effectively and fully understood. How a medical authority instructs and obliges patients in written information is also unclear.

While the number of radiographic examinations performed globally increases year on year, studies consistently show that patients lack basic knowledge regarding the commonly-performed exams and show very poor understanding of the concomitant risks associated with radiation. There is, then, a pressing need to investigate radiography patient information in order to better understand why, and where, it is less effective.
\end{abstract}

This thesis applies three approaches common to the field of corpus linguistics to uncover some of the lexical characteristics of patient information for radiography. The approaches used in this thesis are a keyword extraction, a lexical bundles analysis and an investigation of modal verbs used to express obligation.

The findings suggest that patient information for radiography possesses characteristics more common to academic prose than conversation, although the high informational content of the register goes some way to explaining this and suggests that the reliance on these structures may, to a certain extent, be unavoidable. Results also suggest that the reliance on should to oblige and instruct is problematic as it may cause interpretation problems for certain patients, including those for whom English is not a primary language.

Certain other characteristics of patient information revealed by the analyses may also cause comprehension, and while further research is needed, none of these characteristics would be evaluated as problematic by standard readability measures, furthering doubts about the suitability of such measures for the evaluation of medical information. 


\section{Table of Contents}

1. The case for a corpus-based, lexical analysis of patient information for radiography 1

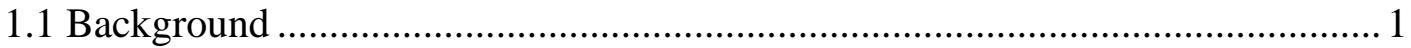

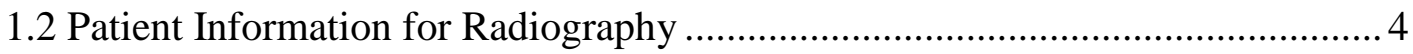

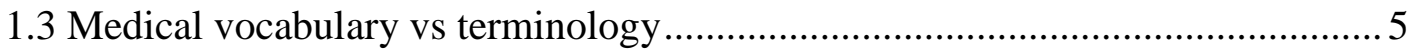

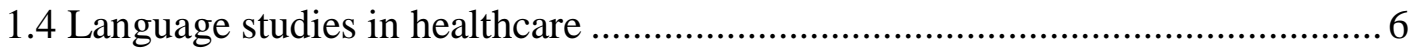

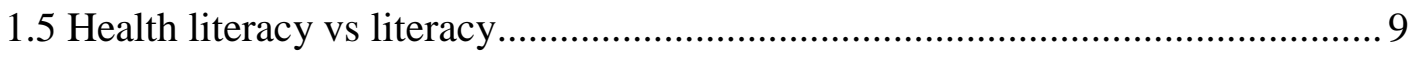

1.6 The importance of information in healthcare .............................................. 10

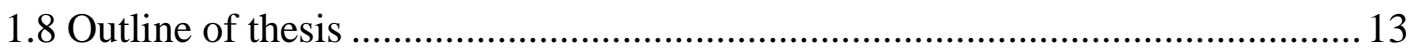

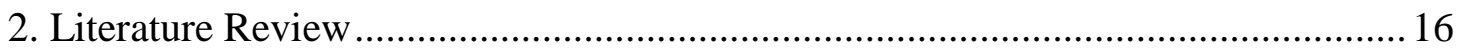

2.1 Academic medical registers in the literature ................................................ 18

2.1.1 Research papers and case notes: genre analysis.................................... 18

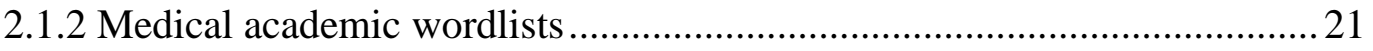

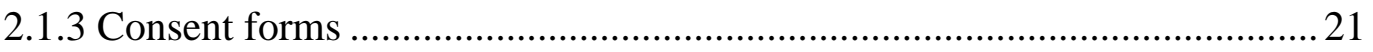

2.1.4. Chronic condition information and biomedical discourse ....................... 24

2.2 The PIL: Pharmaceutical patient information leaflet .....................................26

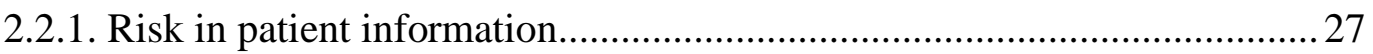

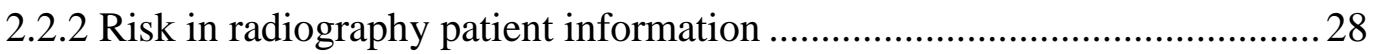

2.3 The applied linguist and healthcare communication....................................... 32

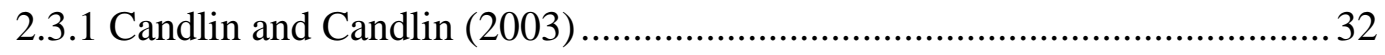


2.4 Corpus approaches to healthcare discourse analysis....................................... 38

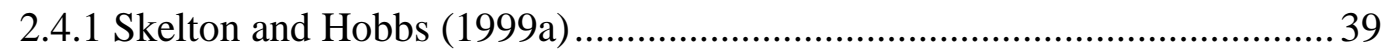

2.4.2 Adolphs, Brown, Carter, Crawford and Sahota (2004)............................ 43

2.4.3 Seale, Ziebland and Charteris-Black (2006) …........................................ 46

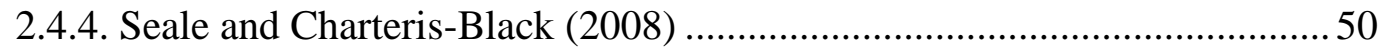

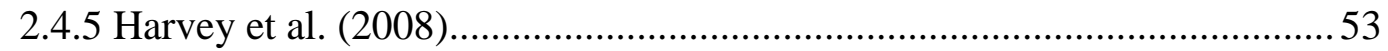

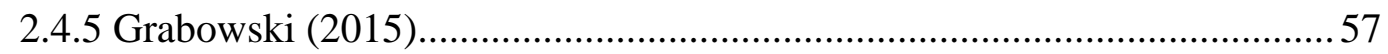

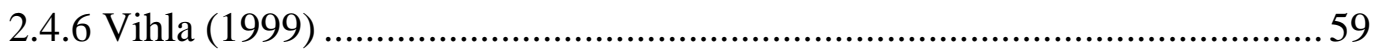

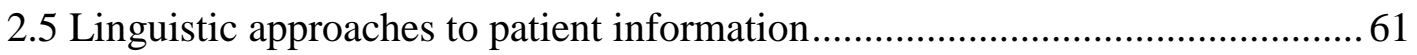

2.5.1 Clerehan, Buchbinder and Moody (2005)............................................. 61

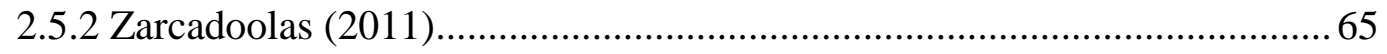

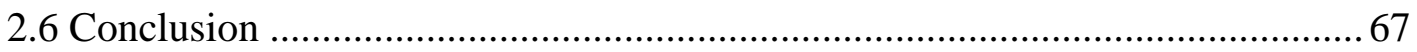

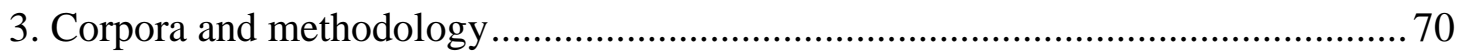

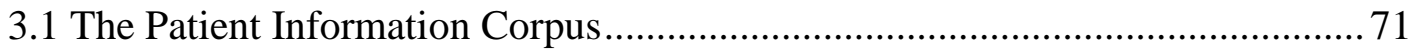

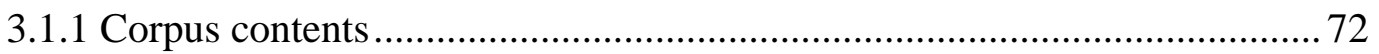

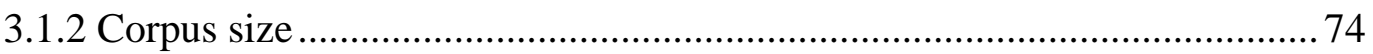

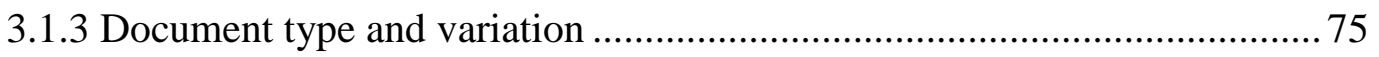

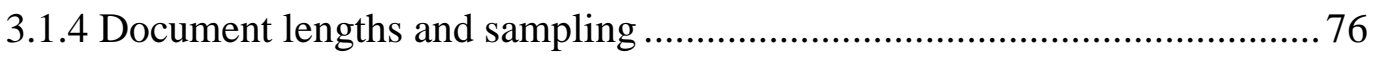

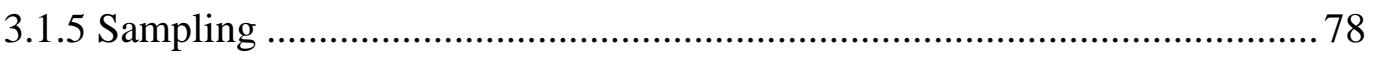

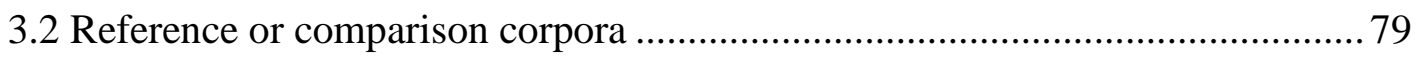


4.3 Methodology 
4.5.1 Legal

4.5.2 Medical Professional

4.5.3 Treatment/therapy

4.5.4 Information

4.5.5 General

4.5.6. Patient-friendly vocabulary

4.6 Conclusions

5. Lexical Bundles in Patient Information

5.1 Lexical bundles in the literature

5.2 The discourse function of lexical bundles

5.3 Methodology

5.4 Results

5.4.1 Overall distribution of lexical bundles in patient information 165

5.4.2 Structural type 166

5.4.3 Discourse function of bundles 167

5.4.4 Frequency of structural types. 169

5.5 Discussion 169

5.5.1 Structural types. 
5.5.3 Stance bundles in patient information

5.5.4 Discourse Organising Bundles

5.6 Conclusions

6. Modal verbs as instructions in patient information

6.1 The importance of instructions in patient information

6.2 Why modal verbs?

6.3 Modals and semi-modals in English

6.3.1 Modal meanings

6.3.2 Personal vs logical modal meaning

6.4 Instruction, obligation or directive?

6.5 Frequency of modals in English

6.5.1. Frequency of deontic modals

6.5.2 Diachronic change in deontic modal use

6.6 Methodology

6.6.1 Patient information corpus

6.6.2 Comparative corpus 1: consumer advice

6.6.3 Comparative corpus 2: General radiography

6.6.4 Search criteria.

6.6.5 Methodological steps 1-3

6.7 Results 
6.8.1 General frequency of modal verbs in patient information

6.8.2 Deontic modals in consumer advice and general radiography.

6.9.1 Need to

6.9.2 Must and have to

6.9.3 Should

6.9.4 L1 and L2 uses of deontic modals in healthcare contexts 226

6.10 Conclusion 230

7. Conclusions

Appendices 246

Appendix A 246

Appendix B

References 


\section{List of Tables}

Table 1 Corpus studies of medical discourse presented in section 2.4

Table 2 Count of imaging activity in England, on NHS Patients, January 2016 to January 2017

Table 3 The contents of the corpus of patient information for radiography .74

Table 4 Exclusion criteria applied to the extracted lexical bundles 101

Table 5 Structural classification of lexical bundles in academic prose adapted.

(Biber et al. 1999, p. 1015-1024) 102

Table 6 Functional classification of lexical bundles (Biber, Conrad \& Cortes, 2004, p. 384-388) 105

Table 7 First 50 keywords with BNC as the reference corpus. 118

Table 8 Top 50 keywords using the general radiography corpus

Table 9 Semantic classification of keywords

Table 10 Distribution by word class in keyword lists.

Table 11 1st 10 keywords with both BNC and radiography reference corpora 130

Table 12 Frequency data for Medical professionals in patient information 140

Table 13 Publications with sex and gender differences in the most frequent clinical entities (Regitz-Zagrosek, 2012)

Table 14 Common collocates of please in patient information for Radiography. ..... 150

Table 15 Patient friendly keywords with their medical equivalents 151

Table 16 Exclusion criteria applied to extracted list of lexical bundles 164

Table 17 Distribution of structural types of lexical bundle in patient information after Biber et al. (1999) 
Table 18 Discourse function of bundles in patient information for radiography....... 168

Table 19 Deontic modal frequency in GB, Aus and US English ( Collins, 2009).... 202

Table 20 Deontic modal verb frequency in the three corpora .209

Table 21 Distribution of epistemic and deontic should in patient information....... 223

Table 22 Survey of deontic modal use in radiography materials: participant info .... 226

\section{List of Figures}

Figure 1 Adding text to the corpus. 83

Figure 2 Compiling corpus 84

Figure 3 Word Sketch showing results for 'pain' .85

Figure 4 Extracting a list of keywords 93

Figure 5 Keyword list .95

Figure 6 Options selected to produce 4-word bundles 99

Figure 7 Taxonomy of discourse categories, after Biber et al., 2004a; Conrad and Biber, 2005. 163

Figure 8 Frequency of common modal and semi-modal verbs in patient information 208

Figure 9 Frequency of deontic modal verbs in the three corpora 209

Figure 10 L1 and L2 Speaker difference in use of modals 228 
1 1. The case for a corpus-based, lexical analysis of patient

2 information for radiography

\section{$3 \quad 1.1$ Background}

4 Medical patient information is the cornerstone of the policy of shared decision making

5 in medicine, a key aspect of patient centred healthcare in many countries, particularly

6 the UK, in the $21^{\text {st }}$ century. Written information plays an increasingly vital role in the

7 relationship between practitioner and patient and this relationship has been of growing

8 interest to researchers from a variety of disciplines, including applied linguistics, for

9 the past three decades. And not only the practitioner-patient relationship: research into

10 medical discourse more generally continues to increase, and as the number of studies

11 has grown, so the scope of inquiry has widened. The term 'practitioner' now

12 references a wider range of healthcare professional, including nurse, dentist, surgeon,

13 nutritionist or radiographer, and not solely the much-studied, general practitioner (GP)

14 (i.e. family doctor) who had dominated earlier studies. Areas of interest to language

15 researchers have spread beyond the academic and the relational to include a great

16 variety of topics such as public health campaigns (Buckton, Lean \& Combet, 2015;

17 Zarcadoolas, 2010), online healthcare information-seeking (Harvey et al., 2008;

18 Mullany, Smith, Harvey \& Adolphs, 2015), metaphor in medical talk (Nerlich \&

19 Halliday, 2007; Semino, Demjen, Hardie, Payne \& Rayson, 2018) and patient

20 narratives (Moore \& Hallenbeck, 2010).

The availability of user-friendly corpus software, such as WordSmith Tools

23 (Scott, 2017) and Sketch Engine (Kilgarriff, Rychly, Smrz \& Tugwell, 2004) is also a

24 factor in the growth of studies of healthcare discourse. The ease of use of software has

25 made the building, analysing and comparison of corpora relatively straightforward

26 with much of the work being automated. This is particularly the case for written

27 corpora, of course, as collecting spoken data in a medical context is still a complex

28 process with ethical issues and a raft of permissions that need to be granted before the

29 data collection, and transcription, begin. 
An additional factor behind the increase in healthcare discourse studies is the

31 huge rise in the use of the internet to search for healthcare information, to talk about

32 healthcare both as a patient and as a professional, and to practise medicine. The e -

33 health or digital health market, which has a projected growth rate of more than $15 \%$ in

34 the US, is driven by the prevalence of chronic diseases and government initiatives to

35 deploy e-health (Grand View Research, 2018). In the UK, more than $80 \%$ of adults

36 use the internet regularly with just over half reporting that they use the internet to

37 search for health-related information, a figure that has grown by more than $30 \%$ over

38 the preceding decade. (Office of National Statistics, 2018). The use of the internet for

39 health reasons is of particular interest to language researchers who have studied

40 patient-professional exchanges, including medical advice sites (e.g. Harvey, Locher \&

41 Mullany, 2013) as well as patient-patient communication via forums (e.g. Angouri \&

42 Sanderson, 2016; Seale, Ziebland \& Charteris-Black, 2006).

In spite of the growing interest in healthcare discourse studies, however, and

44 despite its ubiquity and importance, written patient information has received scant

45 attention from applied linguists.

46 The lack of attention paid to medical patient information may, in part, be

47 explained by the fact that spoken medical discourse receives more attention in the

48 literature, which is understandable, says Clerehan (2014, p 212) as spoken discourse is

49 'arguably the most salient, significant and principal mode of healthcare

50 communication'. The lack of attention may also be explained by the fact that the use

51 and visibility of patient information in many countries is a relatively recent

52 phenomenon. In Switzerland, where I live, there is currently very little printed

53 information available of the kind that is ubiquitous in the UK. Switzerland is a country

54 where medical care is still paternalistic in nature, however, (Lucassen, 2015) along

55 with many countries in eastern Europe (Simek, Krizova \& Zamykalova, 2012) and

56 Asia (Claramita, Nugraheni, van Dalen \& van der Vleuten, 2013). The value of an

57 informed patient (and thus by extension, the availability of patient information) is

58 likely to be perceived less favourably in such countries.

59 In the UK and the US, however, patient information has been visible,

60 considered important and discussed for many decades, making the lack of research 
61 from language researchers puzzling. The first official advice in the UK for writers of

62 patient information first appeared in 1962. This booklet, the result of research by the

63 King Edward's Hospital Trust, and presented as a style guide, contained advice on the

64 writing of information for patients who were being admitted into hospital. (King

65 Edward's Hospital Trust, 1962). Interestingly, the booklet referenced earlier reports

66 from the early 1950s on the importance of giving patients information and what

67 patients needed to know. The reasons behind informing patients and the advice

68 regarding the tone in which the information should be presented looks very similar to

69 contemporary guidelines issued by the NHS and other authorities on patient

70 information such as the Patient Information Forum:

For many people, admission to hospital is counted amongst the major events of their lives. Though most patients later recall their stay in hospital with gratitude and relief, beforehand they all too often view the prospect of admission with uncertainty and apprehension. It is largely to help dispel these fears, and to prepare patients for the unfamiliar hospital world, that information booklets are issued. It is clear too that some authorities consider the booklet is not just a means of giving information but also a way of helping to establish rapport with a patient in a manner that is warm and understanding, rather than patronizing or pompous. (King Edward's Trust, 1962, p 3)

The booklet does not refer to language directly, though does suggest that pictures and cartoons are included, along with a map, contact information and a friendly foreword, saying that 'this feature (or its absence) usually sets the tone of the booklet as a whole.' (p 6). The emphasis is very much on making the text attractive and readable for the patient. It is also interesting to note that the practice of asking patients and staff (not only department heads) to comment on the patient information before it was printed was being practised by a number of hospitals in the UK in the early 1960s. In 2018, however, patient involvement in the production of patient information is still not universal.

Patient information is not, then, a recent phenomenon in the UK, or the US. Given the importance of information for health outcomes, and the on-going concerns relating to its effectiveness and comprehensibility, the relative lack of interest from applied linguists is surprising. 


\section{$94 \quad 1.2$ Patient Information for Radiography}

95 This doctoral thesis concerns itself with a specific kind of patient information from a

96 specific medical field: procedural patient information for radiography. This type of

97 patient information is specific to a particular radiographic exam, such as computed

98 tomography (CT) or magnetic resonance imaging (MRI), or it may relate to a medical

99 procedure that involves the use of a radiographic technique, such as angiography.

100 There is a pressing need to explore the language used in radiography patient

101 information, with the objective of improving the patient's understanding of the topic,

102 as studies show that, in spite of the increasing number of examinations being

103 performed, particularly CT scans (e.g. Thurley, Crookdake, Norwood, Sturrock \&

104 Fogarty, 2017), patients do not understand the differences between common

105 radiographic examinations, are unable to say which exams use radiation and, almost

106 certainly as a result of this lack of knowledge, they show an alarming lack of concern

107 for radiation risk. (e.g. Singh, Mohacsy, Connell \& Schneider, 2017). The lack of

108 understanding of the technology and associated risk may be compounded by the

109 complexity or even non-availability of printed information materials.

110 I have taught English to radiographers in Switzerland for a number of years,

111 and in this time I have often used procedural patient information from the UK and the

112 US as teaching material. Patient information lends itself well to English as a Second

113 Language instruction as the text is generally written as if it were a conversation, with

114 questions and responses relating to a patient's experience of a range of radiographic

115 examinations. CT, MRI, ultrasound and plain X-ray are the most common. This

116 written conversation is assumed to resemble what occurs in the radiography suite in a

117 hospital, and thus is useful for presenting vocabulary and themes student

118 radiographers will need in the workplace. At the beginning of this doctoral process,

119 then, I was very familiar with patient information for radiography. I was also aware

120 that writers were expected to follow certain guidelines relating to vocabulary and

121 structure, although my reading of research had also shown me that there was, in fact,

122 great variation in healthcare materials relating to the content and accuracy of the

123 information, and also to the perceived complexity of the information and thus its

124 comprehensibility. 


\subsection{Medical vocabulary vs terminology}

126 In this thesis I use the terms medical vocabulary and (medical) terminology

127 synonymously. I believe that referring to the kinds of words used in medical

128 interactions as either general or medical is an unhelpful and overly simplistic

129 dichotomy. It is more helpful to view this vocabulary as being situated on a

130 continuum, with medical nomenclature (i.e. technical terms characterised by Latin and

131 Greek terms and medical abbreviations) at one end, and very general vocabulary at the

132 other. In between we find other categories that we may refer to as semi-technical

133 vocabulary and lay technical, and general items at the other extreme. These are very

134 useful terms to categorise vocabulary, particularly if our attention is on the vocabulary

135 itself, rather than the use or understanding of it in medical interaction. How a word is

136 used and understood in medical interaction varies from person to person however,

137 suggesting that a word can belong to a number of categories - fully technical, semi-

138 technical and even lay technical - depending on how the word is understood by the

139 person using it.

140 Patients often use medical vocabulary when they are talking about their

141 condition, though they sometimes use it in different ways to the professional. Chronic

142 is a good example of this, meaning 'long-lasting' for a medical professional and 'bad'

143 for some speakers of English. Lay people may also use terms differently because they

144 emphasise the experience of the condition or symptom. Additionally, it may also be

145 the case that patients, with their individual subjective experience of a condition always

146 use medical terms differently to the professional, and that just as personal experience

147 of a condition varies from one person to another, so do the meanings attached to

148 words.

149 Professionals, too, may use the same word but their understanding of the word 150 can differ, depending on their specialism and understanding of the field: a consultant

151 psychiatrist who researches the condition will understand schizophrenia in a different

152 way to the GP, while the lay person with schizophrenia has an understanding of the

153 issue of a very important, but different, kind. (J Skelton, personal communication, 23

154 October 2018). 
155 Given the complexity of medical vocabulary, and the importance of improving the

156 comprehensibility of published healthcare materials, it is no surprise that the use and

157 comprehension of medical vocabulary has, to date, been the principal area of interest

158 for language researchers.

\subsection{Language studies in healthcare}

160 Studies from applied linguistics of healthcare communication are very valuable

161 because they 'offer a means of making sense of some of the complexities of

162 healthcare: exposing beliefs and practices that might be taken for granted or

163 overlooked altogether' (Harvey and Koteyko, 2013, p 2). Most language studies (from

164 applied linguistics and other fields) have focused on the use of specialised, medical

165 vocabulary (e.g. Bourhis, Roth \& Macqueen, 1989; Skelton \& Hobbs, 1999a; 1999b),

166 while the differences in lexical style of doctors and nurses (Bourhis, Roth \&

167 Macqueen, 1989; Collins, 2005) and the lexical challenges facing medical

168 professionals trained abroad (Bosher \& Smalkoski, 2002; Cameron, 1998; Dahm,

169 2011) have also been of interest.

170 Earlier studies of spoken medical discourse, many using the approach of

171 conversational analysis, tend to focus more on the practitioner, often a doctor rather

172 than any other health professional. Initial findings that doctors use a lot of complex

173 medical vocabulary with patients, in spite of their perception otherwise (e.g. Bourhis,

174 Roth \& MacQueen), contrast with other, later studies, e.g. Skelton \& Hobbs, 1999a,

175 who find that doctors are, in fact, generally aware of their use of medical vocabulary

176 and are able to explain relevant terms for their patients (though it is notable that in

177 Skelton \& Hobbs, 1999a, it is they, the doctors, who decide what needs explaining).

178 The narrow focus on the practitioner in healthcare communication studies began to

179 widen to include the patient, and many studies since are interested in the

180 comprehension of, and the impact of, medical vocabulary on the patient. Most

181 research comes to the conclusion that patients understand far less than doctors think

182 they do (e.g. Chapman, Abraham, Jenkins \& Fallowfield, 2003; O’Connell, Hartridge-

183 Lambert, Din, St John, Hitchins \& Johnson, 2014). Even terms as significant in

184 meaning as benign and malignant fail to be understood by as many as a third of cancer

185 patients (Chapman et al., 2014). It is possible, conclude Chapman et al. (2014), that a 
substantial proportion of the public do not understand the language used in consultations, nor can their knowledge of even basic anatomy be assumed, with serious implications for the success of many medical interactions. An early study by Boyle (1970) found similar results to Chapman et al. (2014) regarding patients' ignorance of anatomy, which suggests that internet use has not resulted in an increase in this particular type of knowledge. Ignorance of medical vocabulary in general has been reported in many other studies and for other languages (e.g. Blackman \& Sahebjalal, 2014; Hayes, Dua, Yeung \& Fan, 2017; Pieterse, Jager, Smets \& Henselmans, 2013).

While studies in disciplines other than applied linguistics and health communication continue to focus on what a patient does not understand, (e.g. Cherla, Sanghvi, Choudhry, Liu \& Eloy, 2012; Hansberry, John A, John,

E, Agarwal, Gonzales \& Baker, 2014), a handful of more recent studies by linguists and healthcare communication researchers have focused on what patients do understand, and how they use the terms that they know ( Fage-Butler \& Jensen, 2016; Koch-Weser, de Jong \& Rudd, 2009; 2010). These studies reveal a considerably more complex picture than the standard understand/do not understand dichotomy.

There is growing evidence that many patients, particularly those living with a chronic condition, use medical terms, and as they become better informed about their condition, these terms are used with greater frequency (e.g. Fage-Butler \& Jensen, 2016). The identity of the 'expert' patient, a phenomenon that reflects a growing confidence among health consumers, a technological society and the ready availability of health information, has been the focus of a number of studies (Fox \& Ward, 2006; Fox, Ward \& O'Rourke, 2005).

Koch-Weser, de Jong \& Rudd (2009) focused on the words used by both patient and professional, finding that while some patients use medical terms as often as their doctor during a consultation they rarely use the same words. The study found that patient medical vocabulary tends to cluster in the history-taking section of the consultation, which is not surprising as this also tends to be the moment when the patient has an opportunity to speak. Professionals, on the other hand, use medical vocabulary throughout the consultation. Further research may reveal whether patients 
217 do not use medical vocabulary elsewhere in the consultation simply because they are

218 not speaking, or whether they are only able to use it when they are explaining to the

219 doctor the reason for the appointment. If this is the case, doctors should not assume

220 that patients understand everything that is being said during the consultation,

221 irrespective of their accurate use of medical words in the history-taking part.

222 Additionally, patients who are not able to use terminology in the history-taking part of

223 the consultation may well have a lower health literacy level, which the doctor will

224 need to be aware of and adapt to. As Koch-Weser, Rudd and DeJong (2010) say:

For their part, patients must express themselves clearly to participate actively in decision-making. Patients' success in describing their symptoms accurately depends in part on the sophistication of the vocabulary they can call on. Thus, measures of word use can offer insight into their "expressed literacy level." By extension, such measures may also indicate the vocabulary that patients are likely to comprehend. (p 591)

These findings make it all the more important to train doctors to allow the patient to speak during the history-taking phase without interrupting as such an approach can yield very useful information.

As we have seen, while some patients do use medical vocabulary, studies also show that patients do not always use words with the same meanings as doctors. There are different explanations for this, though one reason is the different understanding practitioners and patients have of a word, as discussed above. (Dahm, 2018; 2011; Hadlow \& Pitts, 1991; Ong, de Haes, Hoos \& Lammes, 1995). Professionals and patients can respond differently to words, which is likely a response to the connotations of the words. This seems particularly the case with words that reference mental illness such as depression, along with words for body size, weight and the concept of obesity (Mullany, Smith, Harvey and Adolphs, 2015; Ogden et al., 1999; Tailor \& Ogden, 2009). The emotional response to a word's connotations are not straightforward, however. Tailor and Ogden (2009) found that while doctors prefer to use euphemisms rather than the term obese with their patients, patients who were truly obese found the euphemism more upsetting to hear. On the other hand, patients who were not obese (when their BMI was measured) felt more anxious and upset on hearing the term obese than they did when the euphemism was used. The authors conclude, perhaps a little glibly, that a 'GPs choice of term therefore needs to reflect 
250 whether they want the patients to be upset or whether they want them to accept the 251 seriousness of their problem.' (2009, p260).

The fact that different meanings and connotations can be attached to words is

253 further evidence that the traditional dichotomy of technical and semi-technical - and

254 the simplified view of medical jargon versus general language - fails to capture the

255 very varied characteristics of medical vocabulary. In a recent study, Fage-Butler and

256 Jensen (2016) developed five categories of medical terms, using them as a framework

257 to evaluate online forum interactions by patients with chronic conditions. Patients in

258 the study were found to use a great variety of terms from all of the categories, leading

259 the authors to cautiously recommend that doctors use an adaptive approach to medical

260 terminology during consultations, adaptive to the knowledge of their patients.

261 The idea of adapting to the knowledge and health literacy of the patient -

262 which may be higher or lower than the professional initially believes - relates to the

263 idea of tailoring written information to the patient, an approach that studies show is

264 generally more effective than a one-size-fits-all approach to information (Jensen,

265 King, Carcioppolo, Davis, 2012; Lustria et al., 2013.) The idea of tailoring

266 information will be discussed in greater detail in Chapter 4. As it currently stands,

267 however, much of the written procedural information produced by and for hospital

268 departments in the UK is not tailored. General patient information guidelines are

269 applied to all texts and the aim seems to be the simplification of information, for all

270 patients, irrespective of their status.

\section{1.5 Health literacy vs literacy}

272 The significant implications of not understanding, and the negative impact of poor

273 understanding on patient outcomes has motivated the very many studies of written

274 health materials (all from fields other than applied linguistics) that focus on patient

275 comprehension and the readability of materials. While different readability tests use

276 different measures, they generally count word length in syllables and/or sentence

277 length to assess the complexity of a text. However, most readability tests cannot

278 differentiate between a monosyllabic or two syllable medical word and a general term:

279 sacral, benign and ructus could be assessed as more readable than operation or 
corridor. Another problem with the concept of readability is that it relates to literacy.

281 Literacy, however, is not the same as health literacy. As Zarcadoolas says (2011),

282 health literacy is complex and multifaceted, requiring much more than the ability to

283 read simplified text. Just how complex and multifaceted is made clear in the following

284 paragraph from the US Department of Health and Human Services, who define health

285 literacy as:

The degree to which individuals have the capacity to obtain, process, and understand basic health information and services needed to make appropriate health decisions. Health literacy is dependent on individual and systemic factors: Communication skills of lay persons and professionals; Lay and professional knowledge of health topics; Culture; Demands of the healthcare and public health systems and the Demands of the situation/context

Health literacy affects people's ability to: Navigate the healthcare system, including filling out complex forms and locating providers and services; Share personal information, such as health history, with providers; Engage in selfcare and chronic-disease management [and] understand mathematical concepts such as probability and risk. (Department of Health and Human Services. n.d)

To close the comprehension gap between health messages and the public, Zarcadoolas (2011) calls for `a richer, more theory-based understanding of text structures and functions, along with other powerful constructs, including cultural appropriateness, relevancy and context'. (p 338). This thesis shares the view, expressed by Zarcadoolas (2011) and a handful of other researchers (e.g. Clerehan, Buchbinder \& Moody, 2005; Rubin, 2014), that standard readability measures are inappropriate tools for the development and evaluation of patient information materials. The 'theory-based understanding of the structure and function of text' that Zarcadoolas (2011, p 338) refers to is, I believe, an area of knowledge that applied linguistics research, including the research reported in this thesis, can certainly contribute to.

\section{$308 \quad 1.6$ The importance of information in healthcare}

309 Turning now to the final motivating factor for this doctoral research: the importance

310 of information in healthcare. The ultimate objective of studies in healthcare

311 communication must be to improve patient satisfaction and health outcomes, and there

312 is plenty of evidence of the role that being or feeling informed has in improving

313 clinical and non-clinical outcomes (Sheard \& Garrud, 2005; Coulter \& Ellins, 2007; 
314 on knowledge (Maggs, Jubb \& Kemm, 1996) and of the positive relationship

315 information has with compliance and adherence to treatment programmes

316 (Boundouki, Humphris \& Field, 2004;). I believe that studies of the language used in

317 healthcare communication, such as those reported in this chapter, can help make

318 information effective, whether it be written information or spoken.

319 Information must be comprehensible in order to be effective. And while a

320 better understanding of the complex nature of medical vocabulary is fundamental to

321 healthcare communication studies, we should not forget about general language.

322 General language makes up a sizeable amount of the language of patient information -

323 after all, most complexity and terminology should have been removed if guidelines

324 are followed - and general language is usually perceived to be transparent in meaning

325 and to cause few comprehension problems. Is this really the case? Until we investigate

326 the characteristics of general language in patient information how can we be sure that

327 it is a valid assumption?

328 It is general vocabulary, then, as used in medical patient information that is the

329 primary focus of this doctoral thesis. My aim is to uncover the lexical characteristics

330 of patient information for radiography through the application of corpus linguistics

331 approaches. The lexical analyses I conduct may well reveal the kinds of hidden beliefs

332 and practices that Harvey and Koteyko (2013) refer to, they may reveal the underlying

333 discourses and, additionally, they may also uncover linguistic aspects that may play a

334 significant role in the comprehension of the text.

A variety of applied linguistic approaches have been used in the investigation

336 of healthcare communication, and two of the more common for studying spoken

337 medical interaction have been critical discourse analysis (e.g. Mishler, 1984), a mode

338 of discourse analysis concerned with power relations and ideologies in language, and

339 conversation analysis (CA) (e.g. Drew, Chatwin \& Collins, 2008; Heritage \& Stivers,

340 1999; Jones, 2003) which is an approach that seems particularly suitable for the

341 analysis of patient-provider interaction as it has been described as a direct research

342 embodiment of patient-centredness`(Maynard \& Heritage, 2005, pp. 433-434). In CA,

343 the focus is particularly on the structure of the utterance and turn-taking in the

344 development of the interaction. Written medical discourse, most especially research 
345 papers and their abstracts, has often been investigated from the approach of genre

346 analysis (GA), which, broadly speaking, is an approach to text analysis that defines

347 the lexical parameters inherent in a particular genre, while some studies have

348 combined genre analysis with the use of corpus analysis techniques (Hill Davies,

349 2015). An overview of the approaches used in the investigation of written medical

350 discourse will be presented in Chapter 2.

For the analyses described in this doctoral work, I draw on the field of corpus

352 linguistics, and use the following corpus techniques: a keyword analysis and a lexical

353 bundle analysis. I chose to use the keyword method as it has already been well-used in

354 healthcare communication research and has established itself as an effective tool for

355 revealing the underlying themes in healthcare communication. Some key papers from

356 healthcare communication that utilise this technique, e.g. Adolphs, Brown, Carter,

357 Crawford, \& Sahota (2004), Harvey et al. (2008) and Harvey \& Atkins (2010) are

358 discussed in Chapter 2. As we saw earlier in this chapter, the first style guides

359 produced by the health service in the UK referred to the need to reassure the patient

360 and to 'establish rapport with a patient in a manner that is warm and understanding,

361 rather than patronizing or pompous' (King's Trust, 1962). A keyword analysis can

362 provide a way into the discourse and can show us whether these aspects are still

363 present in the text, or whether there are other issues that have replaced them in

364 importance.

365 The second technique I use is a lexical bundles analysis. Lexical bundles are 366 multiword strings which are generally non-idiomatic, e.g. let's turn to and at the end

367 of. They have been referred to as 'characteristic features of language use in particular

368 settings` (Hyland, 2008, p8) and are very common in both spoken and written

369 discourse. Lexical bundle analysis has already shown itself to be very useful in

370 uncovering the lexical characteristics of a register (e.g. Conrad \& Biber, 2004; Biber

$371 \&$ Barbieri, 2007). A better understanding of the functional characteristics of patient

372 information can be arrived with an analysis of its lexical bundles. We can see, for

373 example, whether the information is written in the conversational style that guidelines

374 encourage writers to adopt, or whether the style of the text might reasonably be

375 expected to develop a rapport with the reader. 
One of the primary reasons for choosing corpus approaches to the analysis of healthcare discourse is that they allow the investigation of very large datasets.

378 Irrespective of the size of the corpus, the use of corpus techniques allows reliable 379 generalisations about language use to be made based on the statistical analyses of a

380 large dataset; comparisons of the frequency of items in one corpus relative to another

381 can be carried out and key words generated, which can give a sense of the nature of

382 specialised discourse. Word lists can be produced for English for Specific Purpose 383 teaching purposes and lexical relationships, such as collocations, investigated. All of 384 these tasks would be difficult, time-consuming or even impossible to carry out without 385 the aid of software.

Frequency information alone, however, is highly unlikely to tell us much about medical communication (Skelton \& Hobbs, 1999a; 1999b). Lists of keywords, for example, can only suggest areas of communicative interest that may be interesting:

389 they are 'pointers which suggest to the prospector areas which are worth mining, but 390 they are not themselves nuggets of gold . (Scott, 2010, p 51). The studies reported in 391 this thesis, then, employ first a quantitative analysis using corpus software followed 392 by a qualitative analysis of the item of interest in the context of the corpus.

In addition to the two approaches described above, a third corpus analysis is 394 described, that of modal verbs in patient information. The modal verbs I use in my 395 analysis are those that are concerned with obligations and instructions and are often 396 referred to as deontic modal verbs. This is a different kind of corpus analysis to those

397 described in the previous paragraph. This analysis involves the prior selection of 398 lexical or grammatical items of interest which are then searched for within the corpus.

399 I was motivated to investigate deontic modal verbs as procedural patient information

400 does not just inform but it also instructs, though how written information uses 401 language to instruct, we know little about. This analysis, and its results, are described 402 in Chapter 6.

\subsection{Outline of thesis}

404 The research in this thesis has been informed by different fields of study: studies from 405 applied linguistics, particularly those that use corpus techniques to describe a language 
variety or register, and corpus studies of healthcare communication. My research has also been informed by studies from fields outside of applied linguistics, such as health literacy, patient education, and social psychology. Health communication is a multidisciplinary field, and applied linguistics interdisciplinary, but it has not always been straightforward to pull together all of the strands that have informed this study.

The literature that has informed my research appears in Chapter 2. This chapter contains key studies of written medical discourse, and studies of healthcare communication, both spoken and written, that have utilised corpus approaches healthcare communication. As we shall see, there have been far more studies of spoken medical interaction utilising corpus techniques than there have been of written registers. The chapter also includes studies that call for a new approach to healthcare communication research and that refer to the methodological difficulties of such cross-disciplinary research. Finally, I present studies that call specifically for a new, linguistic approach to the production and evaluation of healthcare information materials.

In Chapter 3 I describe the software that I used to build and investigate the specific corpora used in my analyses and I present the corpora and methodology used for each analysis. Chapters 4-6 are dedicated to the lexical analyses carried out of key words, lexical bundles and modal verbs for instructions in patient information. Each chapter contains the procedure, results, discussion and conclusions.

In Chapter 7, the thesis concludes with a discussion of the more significant findings from each of the analyses. The findings are first discussed with reference to the questions that motivated the research, namely what they reveal about the lexicogrammatical characteristics of patient information for radiography. I present my conclusions regarding the impact of these characteristics on the comprehension of the patient information, and present my conclusions regarding the appropriacy of standard readability measures to evaluate materials. I also present a summary of the findings that relate to the underlying beliefs and discourses of patient information for radiography that were also revealed by my analyses. The chapter ends with a discussion of the limitations of the research and with some suggestions for further research. Some of these suggestions for future research relate directly to the questions 
437 that motivated my study, while some relate to the themes that emerged during the 438 research process.

439 It is inevitable, perhaps, that an exploratory register analysis such as the one 440 described in these pages, will uncover unexpected themes of interest. These themes 441 are not directly related to the research questions but nonetheless are relevant to the 442 broader topic of healthcare materials and patient-provider communication. A number 443 of such themes emerged during my research. They are presented and discussed 444 generally in chapters 4-6. In chapter 7 they are signalled as areas worthy of further 445 exploration. Any in-depth analysis is beyond the scope of my data, however. 
461 There is a vast amount of research on medical language which, if we were to consider

462 it all relevant for this literature review, would render impossible the task of selecting.

463 This thesis, however, takes the view that medical language is not a homogenous genre,

464 but a genre made up of a large variety of registers, each with its particular lexical and

465 grammatical characteristics. It is the register of written patient information for

466 radiography that is under investigation in this doctoral thesis, while corpus analysis is

467 the methodological approach taken. Register in this thesis is used to mean a `specific

468 language variety associated with a particular configuration of situational

469 characteristics and purposes`. (Staples, Egbert, Biber \& Conrad, 2015, p. 505).

470 This chapter begins with an overview of the literature on medical registers

471 other than procedural patient information, beginning with academic medical English.

472 The research focus here has mostly been on the characteristics of the medical research

473 papers or its sections, on case notes (an academic-professional register), and on

474 producing word lists of academic medical vocabulary.

475 The overview is followed by a summary of the few studies that have

476 investigated the professional register of consent forms. The concerns central to these

477 studies - readability and comprehension - are central to my investigations of

478 procedural patient information. The few studies that look at patient information for

479 sufferers of chronic conditions, such as rheumatoid arthritis and depression, is then

480 summarised. These studies generally aim at understanding whether these healthcare

481 materials are appropriate for the patients and whether their needs are met by

482 information (e.g. Grimes and Ong, 2007) The focus on these studies is particularly on

483 the content, the approach taken is often discourse analysis. The conclusion is that

484 patient information needs to be far more accurate with the information it presents, and

485 to involve the patient, their concerns and their experience far more than it currently 486 does.

My focus then turns to a summary of the literature relating to pharmaceutical 488 patient information leaflets (PILs). There has been a fair amount of interest in PILs 489 from researchers (including applied linguists), possibly because these packaging 
490 inserts are obligatory in Europe, and their content regulated. I believe that many of the

491 themes discussed in these studies are relevant for studies of procedural patient

492 information, such as the one described in this thesis. These themes include readability,

493 complexity and how risk is expressed and perceived.

494 In the second part of this chapter, the broad literature overviews described

495 above give way to more detailed discussions of papers that have particularly informed

496 my research. This section begins with two studies that focus on the importance of

497 cross-disciplinary research. Roberts and Sarangi (2003) present some of the

498 challenges that cross-disciplinary work can bring, while Candlin and Candlin (2003)

499 highlight the importance of real-world outcomes of language research in healthcare.

500 This is a theme that is also present in many of the corpus studies of medical discourse

501 which I present in 2.4. Many of the papers in 2.4 utilise a keyword analysis and I

502 include here studies that have focussed on both written and spoken discourse. Some of

503 the earliest and most important corpus studies of medical language were of spoken

504 communication, e.g Adolphs et al. (2004). Table 1 shows the areas of interest of the

505 corpus studiesI discuss in 2.4 .

506 Table 1 Corpus studies of medical discourse presented in section 2.4

\begin{tabular}{|c|c|c|c|c|}
\hline Section & Authors \& date & Register and focus & Mode & $\begin{array}{c}\text { Corpus } \\
\text { technique? }\end{array}$ \\
\hline 2.4 .1 & $\begin{array}{l}\text { Skelton and Hobbs } \\
\text { 1999(a) }\end{array}$ & $\begin{array}{l}\text { Patient-provider } \\
\text { communication - } \\
\text { consultations }\end{array}$ & Spoken & Concordancing* \\
\hline 2.4 .2 & $\begin{array}{l}\text { Adolphs, Brown, } \\
\text { Carter, Crawford } \\
\text { and Sahota (2004) }\end{array}$ & $\begin{array}{l}\text { Patient-provider } \\
\text { communication - } \\
\text { advice phone lines }\end{array}$ & Spoken & $\begin{array}{l}\text { Keyword } \\
\text { analysis }\end{array}$ \\
\hline 2.4 .3 & $\begin{array}{l}\text { Seale, Ziebland and } \\
\text { Charteris-Black } \\
\text { (2006) }\end{array}$ & $\begin{array}{l}\text { Forum postings } \\
\text { (gender and } \\
\text { cancer) } \\
\text { plus traditional } \\
\text { interviews }\end{array}$ & $\begin{array}{l}\text { Online } \\
\text { written plus } \\
\text { spoken }\end{array}$ & $\begin{array}{l}\text { Keyword } \\
\text { analysis }\end{array}$ \\
\hline 2.4 .4 & $\begin{array}{l}\text { Seale and } \\
\text { Charteris-Black } \\
(2008)\end{array}$ & $\begin{array}{l}\text { Illness narratives } \\
\text { (age and cancer) }\end{array}$ & Spoken & $\begin{array}{l}\text { Keyword } \\
\text { analysis }\end{array}$ \\
\hline
\end{tabular}




\begin{tabular}{|l|l|l|l|l|}
\hline 2.4 .5 & $\begin{array}{l}\text { Harvey et al. } \\
(2008)\end{array}$ & $\begin{array}{l}\text { Adolescent health } \\
\text { communication }\end{array}$ & $\begin{array}{l}\text { Online } \\
\text { written }\end{array}$ & $\begin{array}{l}\text { Keyword } \\
\text { analysis }\end{array}$ \\
\hline 2.4 .6 & Grabowski (2015) & $\begin{array}{l}\text { Pharmaceutical } \\
\text { registers (x4) }\end{array}$ & Written & $\begin{array}{l}\text { Keyword } \\
\text { analysis and } \\
\text { lexical bundle } \\
\text { analysis }\end{array}$ \\
\hline 2.4 .7 & Vihla (1999) & $\begin{array}{l}\text { Modal verbs in } \\
\text { medical registers }\end{array}$ & Written & $\begin{array}{l}\text { Corpus-based } \\
\text { analysis }\end{array}$ \\
\hline \multicolumn{4}{|l}{} \\
*refers to the analysis of lines of text from a corpus. Areas of interest choosing by \\
the researchers and subsequently examined in the corpus
\end{tabular}

The literature review concludes with two studies that focus specifically on

509 healthcare materials and health literacy: Clerehan, Buchbinder and Moody (2005) and

510 Zarcadoolas (2011). Both studies call for a different approach to the development and

511 evaluation of health materials, for a better understanding of what we mean by health

512 literacy and for an approach to the development of healthcare materials that is

513 informed by both the expressed needs of patients and by research from the field of

514 applied linguistics.

\subsection{Academic medical registers in the literature}

516 In medicine, the variety of written registers includes academic research papers, case

517 reports, consent forms, care plans, discharge notes, dietary and lifestyle advice,

518 medical device instructions, handovers, progress notes, imaging reports, injury

519 prevention information, medication information and procedural information. Very

520 few of these have been the subject of any linguistic interest, however. Academic

521 medical writing has been the focus of most interest, with a handful of studies on the

522 language of case reports and consent forms. This are summarised in the following

523 sections.

524 2.1.1 Research papers and case notes: genre analysis

525 In the literature, studies of academic registers predominate, particularly research

526 papers (and their abstracts) (Atkinson, 1995; Nwogu, 1997; Salager-Meyer, 1990; 
1992; 1994) and case reports (Nissen \& Wynn, 2014; Taavitsainen \& Pahta, 2000).

528 Many such studies have been carried out within the field of genre studies and its three 529 research branches: English for Specific Purposes (Swales, 1990; Bhatia, 1993), New

530 Rhetoric (Bawarshi and Reiff, 2010) and Systemic Functional Linguistics (Halliday, 531 1978; 1985; Halliday and Hasan, 1976).

Broadly speaking, these three research traditions all relate to the investigation

533 of the language of discourse communities, though New Rhetoric (NR) is not

534 concerned with L2, unlike English for Specific Purposes (ESP) which is generally

535 concerned with L2 learning and teaching, and Systemic Functional Linguistics (SFL)

536 which is concerned with both L1 and L2 contexts. While an exploration of genre

537 studies is outside of the scope of this thesis, I will present a brief summary of the

538 characteristics of each branch in the section that follows.

539 New Rhetoric, which developed in North America, is particularly focussed on

540 the institutional contexts that surround genres, and the functions of genres within

541 those contexts. SFL, on the other hand, emphasises the function of language in

542 constructing meaning in society. The emphasis in SFL is very much on

543 communication. While SFL has been used less often in Europe as an approach to

544 genre analysis, it is better known in other parts of the world, particularly Australia

545 (e.g. Clerehan, Buchbinder \& Moodie, 2005; Clerehan and Buchbinder, 2006).

546 Clerehan and colleagues take an SFL approach in their various studies of

547 pharmaceutical patient information and in their development of a new framework for

548 the evaluation of patient information. Clerehan et al. (2005) is an important study that

549 will be presented and discussed in more detail later in this chapter.

550 ESP research is particularly concerned with the linguistic features of a text, its 551 organisational, grammatical and stylistic features. Many of the studies overviewed in 552 the section below fall into this research tradition (e.g. Li \& Ge, 2009; Nwogu, 1997).

553 Genre studies within the ESP tradition has been a common approach taken to 554 the analysis of medical research papers and of case notes, the two most academic of 555 registers. Several studies have looked at the structure of the text, with reference to the 556 sections or 'moves' (Swales, 1990) within it (Davies, 2015; Nwogu, 1997; Williams, 557 1999), while other have looked at the historical evolution of the research paper or case 
558 notes (Li \& Ge, 2009; Salager-Meyer, Alcaraz \& Luzardo, 2012). Several studies have

559 focussed on specific linguistic aspects of the text, such as hedging (Salager-Meyer,

560 1994) or modality (Salager-Meyer, 1992; Vihla,1999). How medical research writing

561 and abstracts differ from other disciplines has also been the focus of several studies

562 (Fløttum, 2006; Giannoni, 2010), as have cross-linguistic comparisons (Maci, 2012).

563 Genre analysis within the ESP tradition is concerned with the relationship

564 between the generic characteristics of a text, characteristics which are not fixed but

565 'highly dynamic and closely related to their socio- professional contexts' (Bhatia \&

566 Gotti, 2006). This dynamic variation of the characteristics of a text relates to the

567 purpose of the text, the relationship of writer and recipient, the setting and the

568 communicative events being enacted by the text. The communicative purpose of the

569 text, therefore, shapes the text. The particular interest in academic medical English,

570 indeed, any academic professional subject, relates to the need to train student doctors

571 and scientists to be competent members of what Swales refers a 'discourse

572 community' (Swales, 1990). Learning how to write academic research papers that will

573 be accepted by that discourse community therefore has considerable importance.

574 Genre studies, as we have seen, have investigated the medical research article

575 as a whole, along with its individual sections. Budgell (2011) narrowed the focus even

576 more to investigate the titles of research papers in biomedical research, with a

577 particular focus on randomised control trials (RCTs). A corpus of titles from RCTs

578 was analysed using a comparative corpus made up of titles from 1,000 papers from

579 four generalised medical research journals. Budgell (2011) found that the titles of

580 RCTs are characterised by distinct conventions that include word choice, length and

581 the use of recurrent phrases. Given the role of RCTs in the world of medicine,

582 learning to craft a title according to the convention revealed by Budgell (2011) may

583 well contribute to a paper being accepted for publication.

584 Corpus techniques have predominantly been used for the production of 585 academic wordlists for medical study. These will now be summarised in the following 586 section. 
588 Still within the field of ESP, we move away from small-scale data analysis to corpus

589 linguistic approaches, where large amounts of data are analysed by computer rather

590 than by hand. Here we find a number of studies that focus on the identification of

591 specific lexis for academic medical study. The increasing availability of corpus

592 software over the last 20 years has made the task of producing word lists much easier

593 and over time these word lists have become ever more specific. Coxhead's general

594 Academic Word List (AWL) (2000) was the first such list. Questions relating to the

595 AWL's suitability for specific disciplines, such as medicine, gave rise to an

596 examination of the AWL's coverage of medical research papers (Chen \& Ge, 2007).

597 This in turn led to the creation of the Medical Academic Word List (MAWL) (Wang,

598 Liang \& Ge, 2008) which has been joined more recently by the New Medical

599 Academic Vocabulary List (MAVL) (Lei \& Liu, 2016). The MAVL is around half the

600 length of its predecessor, but with greater coverage. Earlier studies in academic

601 medical discourse (and it must be said, non-academic medical discourse in general)

602 have favoured the physician/doctor over other health professionals and this is also

603 seen in the kinds of word lists that have been produced, where medical students, i.e.

604 doctors in training, are the focus. Nonetheless, academic word lists have also been

605 produced for pharmacology students (Fraser, 2007) and for nurses (Yang, 2015).

606 And what of other registers in written medical English? It transpires that there

607 have been far fewer language studies of written medical registers, other than the

608 academic and pedagogic. Consent forms are one exception. A summary of the

609 literature relating to these important and wordy medical documents now follows

$610 \quad 2.1 .3$ Consent forms

611 Consent forms in medicine are extremely important for they are declarations by the

612 patient that they understand the procedure they will be having and, more pertinently,

613 they understand the concomitant risks. Consent forms have a legal status and their

614 primary purpose is to prove that the patient did understand the risks associated with

615 the procedure and thus the hospital or medical provider are protected from legal

616 recourse. These important documents have been the focus of a handful of language

617 studies (e.g. Ilić, Auchlin, Hedengue, Wenger \& Hurst, 2013; Mayberry \& Mayberry, 
618 2002; Sterling, 2015) with their comprehensibility a primary focus: consent forms,

619 after all, need to be understood before they are signed.

620 It is the presumption of understanding that is the focus of Mayberry and

621 Mayberry's study (2002). The authors, both medical professionals, suggest new

622 approaches (new to the world of medicine, that is) to the testing of comprehension of

623 consent forms, such as a cloze test, and raise as an issue of primary importance, that of

624 the comprehension difficulties faced by patients with functional illiteracy and by those

625 with a language other than English as an L1. The difficulties of comprehending

626 consent forms by L2 speakers of English is also the focus of Sterling (2015).

627 Many studies highlight the length and complexity of the consent form (e.g.

628 Ilić, Auchlin, Hedengue, Wenger \& Hurst, 2013; Pandiya, 2010) and the issue of

629 complexity is central to studies of other medical registers, including patient

630 information leaflets. Readability measures have also been used to assess the

631 comprehensibility of the consent form, though as we will see, literacy measures are

632 problematic when they are applied to medical discourse.

\section{2.1.3.1 Readability measures of consent forms}

634 The limitations to standard readability measures include the fact that these measures

635 do not assess health literacy but literacy. Health literacy and literacy are not the same

636 thing, with the latter relating solely to the ability to read and write. Health literacy, on

637 the other hand, relates to the capacity an individual has to obtain, process and act on

638 health information. Being health literate involves a number of different skills,

639 including social and cognitive skills. An individual can be literate but not health

640 literate. Being health literate is vital for the successful transmission of the message:

641 evidence exists that health literacy and numeracy are far stronger predictors of

642 comprehension of medical information (in the form of a leaflet, for example) than

643 literacy (Hibbard, Peters, Dixon \& Tusler, 2007).

644 Another issue with readability evaluations is the means by which word

645 complexity is measured. Most readability measures cannot distinguish likely-to-be-

646 known long words form unlikely-to-be-known words in the text, distinguishing

647 between them solely by their length. However, some long medical words will be 
648 recognised by many patients as they are part of most people's lexicon, e.g. antibiotic

649 or diarrhoea. These terms are lay technical. It should be remembered, however, that a

650 patient may not use or understand the terms in exactly the same way as a medical

651 professional.

652 Conversely, plenty of rare, technical terms exist that are monosyllabic and thus 653 judged very readable by a standard readability test. Acronyms, e.g. DEXA (dual

654 energy x ray absorptiometry) are a good example of this: used and understood by

655 medical professionals (and often only from the field or speciality) or by the expert

656 patient who is considerably more familiar with them, they are impenetrable to

657 everybody else.

Pandiya (2010) underlines the fact that, while readability contributes to

659 comprehension and promotes a willingness to read the text, readability is not the same

660 thing as comprehension. Pandiya (2010) makes a number of proposals to improve the

661 comprehension of consent forms -which can run up to 20 pages in her experience.

662 Many of the approaches she proposes are those suggested for the development of

663 patient information leaflets, such as those analysed for this doctoral study, and for

664 pharmaceutical information leaflets. They include the use of bullet points and

665 diagrams; the use of general vocabulary in place of medical terms; shorter sentences

666 and frequent paragraphs and the use of active verbs rather than passive. An interesting

667 observation from the study is that in India, where Pandiya works, a standard consent

668 form is translated from English into the very many languages spoken in India. This

669 has resulted in very long documents with literal translations (which cause

670 comprehension problems) and with language that is considerably more sophisticated

671 than the level of many of the readers. While Pandiya (2010) does not refer to the

672 concept of consent being problematic, this may also be a factor: medical consent

673 giving is not common practice all communities and cross-cultural research shows that

674 translations into languages that do not share the concept can result in confusion,

675 embarrassment and even mistrust. (McCabe, Morgan, Curley, Begay \& Ghodes,

676 2005).

677 While the problem of the (in)appropriacy of the level of the language in 678 consent forms remains a concern, there does seem to be some sign of a move away 
679 from sole reliance on the standard readability measure (e.g. Pandiya, 2010;

680 Villafranca, Kereliuk, Hamlin, Johnson and Jacobsohn, 2017). Villafranca et al.

681 (2017) is a particularly good example of this. This study assesses the linguistic

682 complexity of a consent form using a traditional readability measure, in this case the

683 Flesch-Kincaid tool, and also with the Coh-Metrix v3.0 (McNamara, Graesser,

684 McCarthy, Cai, 2014). The Coh-Metric v3.0 software uses the measures of word

685 familiarity and imageability to evaluate the coherence and cohesion of research

686 consent forms. Word familiarity pertains to how familiar the involved words would be

687 to a lay population while average word imageability to how easy it would be for a lay

688 population to visualise the involved words (McNamara et al., 2014). The tool also

689 provides linguistic norms for different types of writing, including science writing, and

690 these norms are sub-divided by grade level, making it a far more sensitive assessment

691 of specialised text than a standard readability measure. Results show, however, that

692 while the measurement tools may change, the appropriacy of the language of

693 healthcare materials continues to be problematic: the researchers considered the

694 language level a problem in all of their 94 research consent form templates, taken

695 from a number of English-speaking countries. All measures exceeded recommended

696 limits and all countries had material which exceeded their own local recommendations

697 for readability.

698 In addition to consent forms, there have been several language studies of

699 health materials that relate to chronic health conditions, such as asthma and

700 rheumatoid arthritis. An overview of these studies is presented in the following

701 section.

\section{2.1.4. Chronic condition information and biomedical discourse}

703 The content and language of health information materials for patients living with

704 chronic conditions, such as osteoarthritis (Grime \& Ong, 2007) and depression (Grime

705 \& Pollock, 2004) have also been investigated. One of the key findings of these studies

706 is that there exists a disparity between the content of the materials and the information

707 that patients say they want to have. Grime and Ong (2007) used a discourse analysis

708 approach to look at the thematic content of six leaflets on osteoarthritis, finding,

709 among other things, inconsistent advice, an emphasis on patient responsibility to 
710 prevent the progress of the disease, and that the leaflets were dominated by a 'disease-

711 discourse' (p. 33). The experience of living with osteoarthritis was backgrounded, if it

712 appeared at all.

713 The finding that written information for chronic conditions often sidelines a

714 patient's experience of living with that condition, prioritising instead a biomedical

715 discourse, was also evident in Grime and Pollocks's study (2004) on a leaflet

716 containing information on depression that accompanied anti-depressant medication.

717 The researchers found that the leaflet used six questions to deliver an overwhelmingly

718 biomedical discourse, while people with depression interviewed by the researchers

719 had a number of questions that remained unanswered by the leaflet. These questions

720 related to self-help, stigma, and to a sense of self, none of which were dealt with at all

721 by the leaflet. The authors conclude that patient information needs to be consistent

722 and accurate with its information and involve the patient, their concerns and their

723 experience far more than it currently does. This is the conclusion of an increasing

724 number of studies (Halkett, Short \& Kristjansen, 2009; Mathers, Chesson \&

725 McKensie, 2009; McCartney, 2013).

726 Involving the patient, as we have seen in Chapter 1, was proposed by the first 727 handbook for patient information produced in the UK nearly 50 years ago, though is a

728 policy that, while frequently recommended, is not always followed by patient

729 information writers. Wright (1999) remarks that involving the patient means

730 collaborating during the production of a leaflet, not simply asking patients at the end

731 of the process whether they can understand the information contained within it.

732 That much patient information concerns itself solely with the biomedical is

733 also seen in studies about studies of patient information: Dixon-Woods (2001) found

734 that of the two principle discourses she identified in over 1,000 papers on patient

735 information, it was the biomedical model that predominated. The idea that patient

736 information, in containing primarily biomedical content, may be falling short of

737 delivering the information that is needed, is relevant for the study described in this

738 thesis. How much procedural patient information for radiography might be described

739 as biomedical or biotechnical in nature? Or, if not biotechnical, what discourse 
740 predominates? The linguistic analyses I perform on my corpus, the results of which

741 are reported in chapters 5-7, may well provide answers to these questions.

\section{2.2 The PIL: Pharmaceutical patient information leaflet}

743 To date, the pharmaceutical patient information leaflet, usually referred to as a PIL,

744 has been the subject of considerably more interest from language researchers than has

745 the procedural patient information leaflet (e.g. Fage-Butler, 2013; Haw \& Stubbs,

746 2011; Hirsh, Clerehan, Staples, Osborne \& Buchbinder, 2009). This may be because,

747 while the content of pharmaceutical patient information in Europe is regulated by law

748 along with the order in which that information is presented, how it is written and what

749 non-obligatory content is included varies considerably, making it an interesting area

750 for the language researcher. It is also the case that, by law, no pharmaceutical product

751 can be marketed without containing an information insert. The fact that medication

752 can kill as well as cure makes it vital that people take their drugs appropriately, which

753 means they need to comprehend the accompanying information leaflets.

754 Many of the findings of language studies of PILs are relevant for this thesis as

755 the healthcare material shares similar objectives: to inform and to instruct. The

756 intended recipient is the patient, rather than the professional, and certain themes such

757 as risks and benefits, are common to both types of information. Some of these studies

758 are overviewed in the next section, while those that have directly informed my

759 research will be presented and discussed in detail later in this chapter.

760 Findings from many studies of PILs suggest that, in spite of the efforts to 761 improve the patient experience through legislation, many consumers do not feel fully 762 informed by them (Haw \& Stubbs, 2011; Raynor, Savage, Knapp and Henley, 2004).

763 This is the case particularly with regard to interactions with other medication along 764 with information regarding side-effects (Dickinson, Raymor \& Duman, 2001).

765 Comprehensibility is the focus of many studies and is a constant concern. In 766 common with patient information in general, many consumers say that they find the 767 language of PILs too dense and complex (Askehave \& Zethsen, 2014). There are even 768 signs that leaflets are more complex now than they were in the past: Askehave and 769 Zethsen (2014) reproduced a survey from 2000 to discover Danish consumers' views 
on PILs. They found that fewer respondents read PILs in 2014 compared to 2000, and

771 fewer respondents agreed that PILs are easy to read. Legislation, say the authors, may

772 well be the explanation for the findings as it has rendered the PIL too regulated and

773 too complex, a view echoed by Wright (1999), who says that listing drug ingredients

774 at the beginning of the leaflets in over-the-counter medications, as per European

775 directives, may well put off some people from continuing to read.

\subsubsection{Risk in patient information}

777 Along with the complexity of the vocabulary and the difficulty reading the text,

778 another central theme in the PIL literature concerns risk: how it is expressed and how

779 it is interpreted. Risk is frequently overestimated by health consumers (Berry, Knapp

780 \& Raynor, 2002; 2006; Pander Maat \& Klaassen, 1994; Pander Maat, 1997), while

781 risk expressed in writing is particularly problematic if imprecise frequency descriptors

782 are used. Imprecise descriptors relate to adjectives and adverbs of frequency, such as

783 seldom, rare or common. The problem with over-estimating risk in the context of

784 healthcare is that patients may be less likely to take their medication as instructed if

785 they feel that side-effects are too likely and too severe. As Berry et al. (2002, p 854)

786 say, 'If people are unable to estimate the risk of occurrence of side-effects, they

787 cannot be expected to make informed decisions about medicinal drug taking.'

EU guidelines issued by the EC Pharmaceutical Committee in 1998 encourage manufacturers to express risk using five frequency descriptors as very rare, rare,

790 uncommon, common, very common. rather than using numeric information. Other

791 adjectives and adverbs expressing frequency, e.g. rarely, sometimes, often, are also

792 used in packaging inserts. Using a word-only approach seems sensible in the light of

793 consumers' (and health professionals') reported difficulty with numeric information

794 and statistics that report risk (Gaissmaier \& Gigerenzer, 2008; Keller \& Siegrist,

795 2007) and the evidence that shows numeracy to be a strong predictor of

796 comprehension of health materials (Hibbard, Peters, Dixon \& Tusler, 2007). Patients

797 with poor numeracy skills will find comprehension more difficult when the risk of

798 side-effects is expressed statistically or in percentages. Nonetheless, research has

799 shown that using the frequency descriptors mentioned above instead of numbers is

800 also highly problematic. The findings of studies investigating the use of qualitative 
801 descriptions of risk, i.e. those using adverbs and adjectives of frequency in place of

802 ratios or percentages, show that patients not only frequently over-estimate risk when

803 they read frequency descriptors (Blalock, Sage, Bitonti, Patel, Dickinson \& Knapp,

804 2016; Berry et al., 2004) they also show great individual variation in their

805 interpretation and express this variation with irregular consistency (Pander Maat \&

806 Klaassen, 1994; Knapp, Gardener \& Woolf, 2015).

807 It is not just the possibility of risk that is over-estimated, however, but how

808 serious that risk is perceived to be: Berry et al. (2002) found that patients reading only

809 verbal descriptors of risk not only over-estimated the likelihood of risk but also the

810 severity of risk, and the risk to health of reported side-effects, while, at the same time,

811 their reported intention to follow the treatment was considerably lower than the

812 control group who were given numeric values only.

813 If both the use of words to express risk and the use of numeric information to

814 express rick can be problematic, what is the effect of using the two approaches

815 together? Using both approaches together is, it transpires, the current advice from the

816 European Medicines Agency (EMA). However, the first study to examine the effect of

817 this approach on estimations of risk finds that this combined approach is also

818 problematic (Knapp, Gardener and Woolf, 2015) as patients consistently, and greatly,

819 over-estimate the risk of side-effects when numeric and frequency descriptors are used

820 together. The authors also confirm earlier findings that numeric information alone

821 contributes to over-estimation which, they conclude, suggests that not only is further

822 research needed on how best to represent risk, particularly on the verbal descriptors

823 which seem to be hold greatest potential, but that government agencies and

824 professional bodies should be very cautious when giving recommendations about the

825 representation of risk in patient information, particularly when they recommend a

826 combined approach.

\section{$827 \quad 2.2 .2$ Risk in radiography patient information}

828 The concept of risk is relevant to my investigations of patient information for 829 radiography, as radiation risk is a topic of central importance, as we saw in chapter 1.

830 While radiography patients express a desire to have information (Mathers, Chesson \&

831 McKensie, 2009; Singh, Mohacsy, Connell \& Schneider, 2017), it seems that most 
832 receive little or no information, including that relating to risk (Ukkola, Oikarinen,

833 Henner, Haapea \& Tervonen, 2017). Ukkola et al. (2017), in their study of the

834 provision of patient information (oral and written) in a radiography department in

835 Finland, found that the quality of information that patients received was poor, and that

836 more than $90 \%$ of patients visiting hospital for a range of radiographic examinations

837 received no information whatsoever about risk, with a similar percentage reporting

838 that they had received no information regarding the benefit of the examination, either.

839 However, even when patients are informed of the risk for computed

840 tomography (CT), (a very common radiographic exam that uses x-rays and thus

841 radiation), and for other radiographic examinations, they show a general tendency to

842 under-estimate the risk (Baumann et al., 2010; Rosencrantz \& Flagg, 2015) and also

843 show a poor understanding of the concomitant risk of radiation exposure. (Baumann et

844 al., 2010; Singh et al., 2017). Singh et al. (2017) found that the majority of patients

845 attending a Melbourne hospital for a range of radiographic examinations did not know

846 that a mammography was a radiation-emitting examination. Disconcertingly, more

847 than half of the patients in the same study expressed no concern about dose and

848 radiation, though the majority also under-estimated the radiation risk of a range of

849 radiation-producing examinations. This finding is in contrast to that regarding risk in

850 pharmaceutical information, where, as we have seen earlier in this chapter, patients

851 generally over-estimate the chance and severity of side-effects. Patients undergoing

852 radiography seem poorly informed about the mechanisms of radiographic modalities

853 and lacking in knowledge about radiation risk. The minimal information a patient

854 receives prior or during a hospital visit may explain the large number of people who

855 express little or no concern about risk (Singh et al., 2017); that is, they are completely

856 unaware that there is any need to be concerned about such a thing. In pharmaceutical

857 information, on the other hand, patients scanning the leaflet see a list of side-effects,

858 common, occasional and rare, bulleted and bolded. Remaining unaware of risk is far

859 less likely. The rising number of CT examinations ordered by doctors in the UK and

860 many healthcare systems, however, makes ignorance about radiation risk a great

861 concern. The rate of CT scans performed in hospital in the US doubled between 2000

862 and 2017, while in the UK, in the same period, there were nearly three times as many

863 CT scans performed (OECD, 2018). 
864 Just as with medication and pharmaceutical advice, patients cannot make

865 informed decisions about their healthcare in the absence of comprehensible

866 information. Clearly, patient information must be available as a matter of priority,

867 whether it be oral or written. The inclusion of risk information for radiography is

868 something that patients say they welcome (Singh et al., 2017) though there have been

869 few studies that focus on how risk information is currently presented in radiographic

870 patient information and how patients understand it.

871 I believe that many of the studies undertaken of pharmaceutical patient

872 information could be undertaken on procedural information, including an assessment

873 of the manner in which risk and benefit - the latter something that patients and

874 professionals are reported to over-estimate (Hoffman \& Del Mar, 2017) - is presented

875 to the patient. Establishing the lexical characteristics of procedural patient information

876 for radiography, as I do in this doctoral study, is the first step.

877 In this chapter thus far, I have presented an overview of the literature on PILs,

878 reasoning that much of the findings of these studies will be relevant to other sub-

879 registers of patient information, including procedural. I have also presented an

880 overview of the literature concerned with other medical registers. As we have seen,

881 many studies have been pedagogic in nature and focussed on the medical research

882 paper or case notes, with a handful of studies related to the consent form and to

883 information for chronic conditions. Given the sheer number of medical research

884 papers published annually (indexed citations at MEDLINE for 2017 number more

885 than 800,000, more than twice those in 1995 (National Library of Medicine, 2018)),

886 and when one considers how many of these papers will be written by people for whom

887 English is an L2, the academic focus in the literature is understandable. Other written

888 registers, however, including procedural patient information, have yet to be explored

889 to any approachable degree by language researchers.

890 I now turn to the second half of this chapter, a presentation and discussion of

891 the studies that have particularly informed this thesis. The literature review that

892 follows is divided into three sections. I begin with a relatively short section where I

893 step back from both the subject of patient information and corpus linguistic studies of

894 healthcare communication in order to consider the importance of collaboration 
895 between applied linguistics and medicine. Some of the methodological issues raised

896 by cross-disciplinary collaboration will also be the focus. Two papers are discussed in

897 this section, both from the same year: Candlin and Candlin (2003) and Roberts and

898 Sarangi (2003).

899 The second section contains studies of the language of medical discourse, both

900 spoken and written, that have used corpus linguistic techniques in their investigations.

901 These studies begin with a discussion of Skelton and Hobbs (1999a). Skelton, an

902 applied linguist, collaborated with a medical professional in this and in subsequent

903 papers (e.g. Skelton \& Hobbs, 1999b; Skelton, Wearn \& Hobbs, 2002) which were

904 written for the medical profession and all published in medical journals, e.g. The

905 Lancet, the British Medical Journal (BMJ) and Medical Education. The literature

906 review continues in this section with a presentation of several studies of healthcare

907 communication that utilise the keyword approach, a corpus technique which has

908 shown itself to be a valuable means of accessing areas of interest in the data, and one

909 applied a keyword approach and a lexical bundle analysis to the investigation of a

910 range of pharmaceutical registers. This latter is one of the very few published studies

911 that have investigated lexical bundles in non-academic medical registers. Here, too, I

912 present one of the very few studies of modal verb use in a range of medical registers.

913 The literature review concludes with two studies that are related specifically to

914 patient information and the way in which it is developed and evaluated. Clerehan et al.

915 (2005) is followed by a study by Zarcadoolas (2013) who calls for a new approach to

916 healthcare materials, one that shows more understanding of how language works, how

917 text is read and the role of pragmatics in making meaning. These papers take the view

918 that using readability measures as the standard evaluation of the comprehensibility of

919 patient information is misguided; both put forward arguments for an approach to the

920 development and appraisal of patient information based on linguistic and

921 communicative principles. I include these studies here because I believe that this

922 doctoral thesis, with its investigation of the underlying linguistic characteristics of the

923 register, can contribute to the kind of knowledge that Zarcadoolas (2013) believes

924 should inform the production and evaluation of healthcare materials. 
926 The papers reviewed in this section underline the value of research by applied

927 linguists in the field of healthcare communication while, at the same time highlighting

928 the importance of looking beyond the field of applied linguistics to other areas - social

929 sciences, medical education, medical communication - where a lot of the research into

930 language and healthcare is being conducted. These papers also refer to the importance

931 of multidisciplinary research and of collaborating with healthcare professionals from

932 the field when conducting research.

933 I found these papers convincing, and, as I have stated previously, much of the

934 reading that has informed this thesis has come from fields other than applied

935 linguistics and throughout the process of conducting research and writing up, I have

936 sought information and advice from medical communication writers and medical

937 professionals. The concerns I have had regarding my ability to conduct self-directed

938 research into medical communication are expressed in 3.2 in my commentary on

939 Roberts and Sarangi (2003). First, though, I present Candlin and Candlin (2003), a key

940 paper that makes clear the value to applied linguists of research - and reading - outside

941 of their field.

$942 \quad$ 2.3.1 Candlin and Candlin (2003)

\section{Summary}

944 In this paper, Candlin and Candlin (2003) present an overview of medical language

945 research and call for applied linguists to broaden their reading to include other

946 disciplines, where considerably more research was being undertaken. The authors

947 highlight some of fields outside of applied linguistics where medical communication

948 research was being conducted, and reference certain journals where it is commonly

949 published (e.g. Social Science and Medicine). They continue by summarising the key

950 themes in the literature (e.g. risk; narratives and interpreting) and by assessing some

951 of the methodological approaches used (e.g. grounded theory; semi-structured

952 interviews and questionnaires). Referencing a number of well-researched studies from

953 various fields, the authors also caution against some of the less methodologically-

954 sound approaches taken, where data is dealt with superficially. The quality of the data 
955 is as important as the methodology used to process it, and it is here, in particular, that

956 applied linguists can make a contribution.

The principal problem referred to in the paper's title, 'Health care

958 communication: a problematic site for applied linguists research', is the outsider status

959 of applied linguistic researchers; it is very rare to find researchers who are both

960 medical practitioners and applied linguists. If applied linguistics really is problem-

961 driven then it should also, say the authors, be outcome-focussed, and thus research

962 should be undertaken wherever possible in collaboration with practitioners and with

963 the end-users in mind. The end-user here is the medical professional or the patient.

964 Achieving a more inclusive and collaborative approach is not easy, though

965 adopting a more open methodological stance and not attempting to fit health data to

966 existing linguistic theories is a start, say Candlin and Candlin (2003). Results, too, can

967 be presented in the language of the practitioners and end-users, while the research

968 questions should not simply address the how, but also the why and to what purpose,

969 echoing Roberts and Sarangi (2003), Adolphs et al. (2004) and Skelton and Hobbs

970 (1999a), all discussed in this chapter, in highlighting the importance of the

971 communicative context and purpose in healthcare language analysis.

\section{Commentary}

973 Candlin and Candlin (2003) is a key paper for benchmarking the developments of

974 research into medical discourse over two decades, both in terms of methodologies

975 used, the disciplinary areas in which the research is conducted and the themes of

976 interest. It is no-longer the case that doctor-patient studies proliferate and there are an

977 increasing number of studies and book-length works on communication in nursing

978 (e.g. Bosher and Stocker, 2015; Henderson, 2016; Lu, 2018; Staples, 2015) though

979 there are still very few studies of the discourse in other healthcare professional fields,

980 such as radiography. It is also the case that the professional voice still predominates.

981 Anton and Goering's book (2015), 'Understanding Patient Voices` is unusual in that it

982 focuses exclusively on patient discourse.

983 There have also been slower developments. Candlin and Candlin (2003)

984 reference just one paper from the English for Specific Purposes Journal, that by Frank 
985 (2000), expressing surprise at the lack of coverage of medical discourse in the journal.

986 In 2018, the journal still devotes comparatively little space to the theme of healthcare

987 discourse, and the papers that are published are generally concerned with academic

988 medical language. Of course, it may be that this is a reflection of the priority given to

989 academic research, which I have referred to earlier in this chapter.

990 To find out more about where studies in the field of medical language are

991 published, I conducted a Scopus search that reveals the majority of studies continue to

992 be published in journals from disciplines other than Applied Linguistics, e.g. The

993 Lancet; Social Science and Medicine; Journal of Advanced Nursing; Patient

994 Education and Counselling. This highlights the need for researchers to continue to

995 head the advice given by Candlin and Candlin (2013) to read broadly. Some progress

996 has been made, however, as there is now a targeted journal dedicated to the subject of

997 communication in healthcare, Communication and Medicine, and a journal dedicated

998 to the field of applied linguistics within the world of work, Journal of Applied

999 Linguistics and Professional Practice. Both of these journals were launched in 2004, a

1000 year after the publication of this paper.

1001 The question is raised in the paper of whether quantitative, questionnaire-

1002 based approaches or qualitative, narrative approaches are more appropriate for

1003 collecting healthcare data. The latter, say Candlin and Candlin, has certain advantages

1004 in that it can reveal `personal constructions of cultural relevancies`(p. 139).

1005 Elsewhere in the paper the authors concede that a combined approach can be

1006 'productive and explanatory' (p. 143), referencing studies that combine discourse

1007 analysis with interviews (e.g. Dijkstra et al., 2002). What is surprising, however, is

1008 that the authors do not mention corpus linguistics or the use of frequency data in

1009 healthcare language studies. Corpus linguistic studies into healthcare discourse were

1010 in their infancy, and this may well be the reason for omitting referencing any of them,

1011 though there were a number of earlier studies into medical discourse, predating

1012 Candlin and Candlin (2013), that make use of both corpus linguistic techniques and

1013 qualitative analyses include Salager-Meyer (1994), Skelton and Hobbs (1999a;

1014 1999b), Skelton et al., (2002) and Ferguson (2001). Only Skelton's research, however,

1015 was concerned with oral interaction and was carried out in collaboration with medical

1016 professionals, something that Candlin and Candlin (2003) feel is essential. This may 
1017 explain the exclusion of Salager-Meyer's (1994) paper and that of Ferguson (2001)

1018 (though not, of course, the exclusion of the work of Skelton and colleagues.)

1019 Roberts and Sarangi (2003), the next paper to be discussed, published in the 1020 same year as Candlin and Candlin (2003), also foregrounds the status of the applied 1021 linguist who conducts research in medical language, and the value and difficulty of

1022 collaboration between language researcher and medical professional, though Roberts 1023 and Sarangi (2013) is primarily a description of the challenges that cross-disciplinary 1024 collaboration can bring.

$1025 \quad$ 2.3.2 Roberts and Sarangi (2003)

\section{Summary}

1027 In this paper, Roberts and Sarangi's (2003) focus was the relations between two 1028 applied discourse analysts (the authors) and medical professionals from the Royal 1029 College of Physicians who had approached the authors for a consultancy. While 1030 collaboration was very much welcomed by all involved in the project and the authors

1031 echo Candlin and Candlin's (2003) call for more outcome-focussed collaborative

1032 work between linguists and professionals, they also concede that this kind of project is

1033 not without difficulty for both parties. Referencing the feedback and comments

1034 received during the write-up of their research for a medical journal, the British

1035 Medical Journal (BMJ), Roberts and Sarangi (2003) describe some of the theoretical

1036 and methodological challenges they experienced, and the reflection that these

1037 challenges prompted.

1038 At the heart of the issue is the 'outsider' status of the sizeable majority of 1039 discourse specialists and applied linguists working in healthcare research, say the 1040 authors. By claiming relevance for their research in a professional field other than 1041 their own, these outsiders face issues of 'identity, roles, authority and credibility` $(p$ 1042 339). All too often, however, this status results in research that is not of direct 1043 practical relevance. What's more, say Roberts and Sarangi (2003), `applied in applied 1044 linguistics is generally used to refer to real-world settings (e.g. the workplace) rather 1045 than real-world outcomes. The traditional relationship between applied linguistic 1046 researcher and their subject is also questioned. Roberts and Sarangi (2003) believe 
1047 that to produce research with useful, practical outcomes, a less imbalanced approach 1048 to collaborative language research is needed, one where every aspect of the study is

1049 developed in conjunction with the research informants: study design, presentation and 1050 dissemination included. This is not as easy as it sounds, however.

1051 A problem occurs when the type of research undertaken, and the language 1052 used to present and disseminate its findings, is perceived to be lacking in rigour and 1053 relevance. Medical research is still very much based on `hard science models, and 1054 where clinical trial research is held up to be the gold standard of scientific enquiry.

1055 The kind of research that discourse analysts engage in, with its qualitative and 1056 ethnographic basis, are 'largely treated with suspicion if not contempt' ( $p$ 341). The 1057 emphasis in medicine is still very much on the 'bio-technical modal' (citing Becker et 1058 al. (1961)), notwithstanding the fact that journals such as Medical Humanities point to 1059 the existence of more humanistic models of medicine. In scientific research,

1060 everything begins with a hypothesis; the open-ended research that the authors were 1061 engaged in did not convince many of their medical collaborators - who, otherwise, 1062 were wholly supportive of the authors' work. Roberts and Sarangi (2003) struggled to 1063 convince their colleagues, for whom hypothesis proving or refuting was the norm, that

1064 'understanding` as a research outcome was a valuable one. Altering their preferred 1065 style of research reporting was also necessary, as the researchers' hedged claims were 1066 considered to be lacking in authority by reviewers more comfortable with the 1067 categorical style of medico-scientific writing. Other issues encountered in the 1068 collaborative process concerned the use of language from the field of discourse 1069 analysis that was not understood by medical professionals, and as a result was 1070 perceived to be inclusive and institutional, creating quite the opposite effect from that 1071 intended. This, and the other issues reported, highlighted the difficulty of

1072 disseminating research findings to an audience unfamiliar with, or even

1073 unappreciative of the methodology and language considered appropriate in the field of 1074 the researchers.

\section{Commentary}

1076 There are many issues raised in this interesting paper about the methodological and 1077 discursive differences that collaboration across disciplines may encounter. I also 
1078 experienced difficulties due to the cross-linguistic nature of the research, though these

1079 difficulties were not evident to me at the beginning of the process. Unlike the other

1080 papers in this chapter, and perhaps unconventionally, I include Roberts and Sarangi

1081 (2003) not as a study that informed my research design or methodology, but as a paper

1082 that provides an explanation for some of the issues I experienced during the research

1083 process, particularly when writing up this thesis. These issues are undoubtedly

1084 common to many, perhaps all, cross-disciplinary studies.

1085 Roberts and Sarangi (2003) also encouraged me to reflect on the assumptions

1086 that I may have had concerning the usefulness of my research, and on my research

1087 outcomes. One of the motivations for this research, as I stated in chapter 1, was my

1088 belief in the importance of patient information for the patient. This research, however,

1089 was self-directed and, while medical professionals were consulted for information and

1090 advice during the research process, the study was not carried out in collaboration with

1091 medical professionals. As I mentioned earlier in this chapter, many studies of medical

1092 discourse that are published for a medical readership have a lack of linguistic

1093 methodological detail that an applied linguist may find a frustrating omission. These

1094 details are omitted as they would be a barrier to comprehension for a medical

1095 readership. It is a fact, however, that as an applied linguist I am the 'outsider' that

1096 Roberts and Sarangi (2003) refer to. I am neither radiographer nor medical

1097 information writer. My findings and my conclusions may not have the value in the

1098 professional world that I would like them to have.

1099 But the reverse is also true. It may be that in exploring studies from outside of

1100 the field of applied linguistics, from social science, medical education and medical

1101 communication, to name but a few, the value of my research may be questioned by

1102 applied linguists. Roberts and Sarangi (2003) found that their discourse analysis

1103 methodology and the qualitative, ethnographic research culture failed to impress their

1104 medical collaborators, whose research culture was hypothesis-based and biotechnical

1105 in nature. Roberts and Sarangi's research culture was even 'viewed with suspicion if

1106 not contempt'. (p. 341). My research has been viewed in a similar way from time to

1107 time, particularly by corpus linguists and those more interested in computational

1108 linguistics, some of whom have stated that applying corpus techniques to language

1109 analysis does not make one a corpus linguist. I am not, however, a discourse analyst, 
1110 nor a sociolinguist, and while the corpus methodologies I have used are well

1111 established methods in a research area that has been referred to as 'applied clinical

1112 linguistics' (Adolphs, Brown, Carter, Crawford \& Sahota, (2004)), it is not a name

1113 that has had much of an impact, perhaps because clinical linguistics is quite a different

1114 field of investigation, with its focus on language disorder. I have suggested that corpus

1115 analysis of healthcare discourse may be part of the field known as medical humanities,

1116 though not all agree. The upshot is, I believe, that being part of a named research

1117 tradition with its own discourse and methodologies (such as keyword extraction),

1118 would help establish the validity of corpus approaches to healthcare communication.

11192.4 Corpus approaches to healthcare discourse analysis

1120 We now shift our focus from the methodological issues surrounding language research

1121 in the field of medicine to the use of corpus techniques in the analysis of medical

1122 discourse.

1123 The first use of corpus techniques for the analysis of medical communication

1124 is generally regarded to be the study by Thomas and Wilson (1996) who compared the

1125 three different approaches of discourse analysis, the use of questionnaires and

1126 'computer content analysis' to the investigation of the interaction between people with

1127 cancer and healthcare professionals. Their findings were that the computerised

1128 approach (a programme called ACAMRIT) did not tell them anything they did not

1129 already know; it did, however, speed up tremendously the investigative work that

1130 analysing the corpus entailed. ACAMRIT is described as an automated content

1131 analyser with a number of additional modules that sets it apart from simpler content

1132 analysers, including parts-of-speech tagging; semantic tagging and conceptual

1133 analysis. This is not a study, however, that foregrounds the practical outcomes of

1134 corpus techniques: its aim was to show what could be done with cutting edge,

1135 computerised analyses of transcribed data, and both researchers were linguists. The

1136 study that follows, however, does foreground medical concerns, and unlike Thomas

1137 and Wilson (1996), Skelton and Hobbs (1999a) was written for medical practitioners

1138 and published in a medical journal, the Lancet. 
1140 Some of the earliest work using a corpus of doctor-patient interaction was carried out

1141 by applied linguist John Skelton and medical colleagues at the University of

1142 Birmingham using Cobuild software.

\section{Summary}

1144 Skelton and Hobbs (1999a) is essentially a descriptive paper on the value of corpus

1145 techniques - the authors refer to it as concordancing - and of combining quantitative

1146 and qualitative approaches for the study of professional language. At that time, such

1147 an approach was entirely new in the context of medicine. The utility of quantitative

1148 data according to the authors is its capacity to capture linguistic aspects of the

1149 consultation that may have been taken for granted and thus remain under-researched.

1150 The importance of qualitative analysis, on the other hand, is that it provides

1151 information about meaning that frequency information alone cannot. As the authors

1152 say 'if words were like numbers, it would be hard to understand why we bother with

1153 both'. (p109)

1154 The corpus was made up of 40 primary care doctors conducting 373

1155 consultations. Skelton and Hobbs (1999a) performed three type of analysis in order

1156 to present to their medical readership the different ways that concordancing can be

1157 used in the analysis of a spoken consultation. The first was a quantitative study of

1158 doctors' use of jargon, the second applied a partial, quantitative operational definition

1159 to the hitherto qualitative concept of doctors' power, while the third analysis aimed to

1160 show the value of the approach in an investigation of the way threats are diminished in

1161 medical consultations.

1162 The first analysis, to investigate the doctors' use of jargon, found that doctors

1163 did not use unexplained words that were considered technical or medical with their

1164 patients. The assumption that doctors did routinely use technical language with their

1165 patients without explanation was simply wrong, say the authors. In the study, when

1166 doctors did use such words, they were often lay technical in nature, such as

1167 paracetamol, which the authors considered non-problematic for the patient. When

1168 more complex words were used, a definition was often provided by the doctor. The 
1169 word was often prefaced with a phrase that signalled the word was about to be defined

1170 or that it would not be, as it was not worth defining. The second analysis concerns

1171 power relations. Operationalising one definition of power imbalance, that of markers

1172 of diffidence connected with social inferiority, the authors select the use of past tense

1173 to reference present concerns. Skelton and Hobbs (1999a) analyse the patients' use of

1174 the past tense in these contexts. 11 uses were found when patients were expressing

1175 worry. The phrase I was wondering was also highlighted as it appears with some

1176 regularity in the corpus, as in I was wondering if you could just give me the sick note

1177 (p. 110). The third analysis found, among other things, that minimisers such as just

1178 and little are used fairly frequently by doctors to encourage the patient and to diminish

1179 a threat, which may be the threat of a potentially serious health condition or the threat

1180 of a potentially embarrassing examination, e.g. can I just have a quick look.

\section{Commentary}

1182 Skelton and Hobbs (1999a) is a study that introduced the potential of corpus analysis

1183 to medical practitioners. Its value is found particularly in the questions that are raised

1184 by the authors in the discussion section, and the suggestions for future research.

1185 Before I come to these, however, a word about the limitations of the paper.

1186 Three different analyses are reported in Skelton and Hobbs (1999a) though

1187 each is short on methodological detail. For an applied linguist this is a little

1188 frustrating. We do not know the size of the corpus, only that it contains 373

1189 consultations. Where detail is given, it is principally related to the statistical

1190 calculations the Cobuild software uses, particularly the Mutual Information (MI)

1191 scores. MI scores relate to the probability of two variables - in this case words -

1192 appearing together. It measures the strength of association between words and is often

1193 used in corpus software programmes such as Sketch Engine to extract collocations. A

1194 t-test was also used by the software. The emphasis on explaining the statistical

1195 processes underpinning the quantitative analysis may well be explained by the need to

1196 explain clearly (avoiding any linguistic jargon) what corpus software does -

1197 remember, such an approach to the analysis of a medical consultation was entirely

1198 new to the world of medicine. The focus on the statistics may also be an

1199 acknowledgement of the attitude to research of the largely medical readership. As we 
1200 saw in the discussion of Roberts and Sarangi (2003), medical and scientific research is

1201 hypothesis-driven, values observable proof and is generally categorical in its claims; a

1202 medical readership can be dismissive or even contemptuous of research approaches

1203 that are not seen to have academic rigour. Statistical information is foregrounded in

1204 this paper, perhaps, to counteract any such suspicion. It would be a more satisfying

1205 paper for an applied linguist, however, if there was more information given about the

1206 methodology and more data examples. We are told, for example, that past tenses were

1207 used to express present worries 11 times, but only one example is given. This is an

1208 interesting area and more examples would have been interesting. We do not know,

1209 either, if one person or 11 people were responsible for the utterances, nor can we

1210 appreciate whether 11 times is to be understood as frequent, as the information needed

1211 to make that decision is not given.

1212 The issue of academic rigour also relates to hedging. Hedging is uncommon

1213 when discussing research findings in medical and scientific research (Roberts and

1214 Sarangi,2003); this may well explain the categorical claim in Skelton and Hobbs

1215 (1999a) which some applied linguists may find overly strong though a medical

1216 readership may not. The claim relates to the first analysis, that of doctors' use of

1217 jargon. The authors claim that the assumption that doctors do use medical jargon in

1218 their consultations is wrong, adding 'for the group of practitioners we analysed' ( $\mathrm{p}$.

1219 110). The addition suggests that the authors also found the claim overly strong.

1220 Nonetheless, my reading of the study tells me quite the opposite. Skelton and Hobbs

1221 (1999a) does show that doctors use jargon. Not only do the practitioners use medical

1222 terms but they often seem to be aware of it, adding a preface containing a signal to

1223 indicate that a complex word is about to be used and that a definition will follow, or

1224 that a definition will not follow, as the practitioners believe that there is need to

1225 translate the term.

1226 The authors also disregard the use of terminology that was considered (by the

1227 authors) to be unproblematic, on the basis that these words were very likely known by

1228 the patient, e.g. paracetamol. There are several issues raised here, some of which the

1229 authors point to in their discussion. The principal issue of whether a term is known by

1230 the patient is a complex question. We have seen already that both professionals and

1231 patients over-estimate the latters' understanding of many words (e.g. Chapman et al., 
1232 2003). How a word is understood by a patient - or by a professional of course - is the 1233 central issue here. When practical, real-word outcomes are the focus, the question of

1234 whether the patient possesses sufficient understanding of a medical word to

1235 comprehend the information being relayed (and to comprehend it more or less as the

1236 professional intends) is more important than how the word itself should be classed i.e.

1237 lay technical or semi technical or fully technical.

1238 The importance of understanding is raised by the authors in the discussion 1239 section who propose further investigation of what we mean by comprehension in a 1240 medical context, and how the training of doctors can be informed by such research.

1241 While I disagree with their conclusions that jargon use in consultations does not cause 1242 miscomprehension (this study does not provide sufficient evidence to reach such a 1243 conclusion), I think Skelton and Hobbs (1999a) convincingly demonstrate that further 1244 investigation of the concept of understanding in medical interaction is warranted. I do 1245 not know whether the readership was convinced by their presentation of concordance 1246 for medical language analysis, however.

1247 The authors propose several areas for further research, including the use of the 1248 language of uncertainty by doctors and patients, of advice and instruction, and of 1249 opportunistic health advice, all of which can be researched using corpus techniques,

1250 while underlining the fact that corpus investigations also lend themselves to the 1251 possibilities of investigating medical interactions from a range of demographic

1252 variables such as age, gender and social class. Some of these variables have been 1253 investigated in the studies that follow, including gender and age. These later studies 1254 are primarily corpus-driven, using comparative data to generate keywords and phrases 1255 that reveal patterns in discourse that may otherwise go unnoticed. Comparative data 1256 was not used in Skelton and Hobbs, 1999a and items were pre-selected for analysis. 1257 While some of these studies that follow were written for a medical readership (e.g. 1258 Seale \& Charteris-Black), others were written primarily for applied linguists (e.g. 1259 Atkins \& Harvey, 2010). The level of methodological detail, therefore, and the 1260 emphasis on real-world outcomes, varies accordingly.

1261 Establishing the role and validity of corpus approaches to healthcare is also the 1262 focus of Adolphs et al. (2004), the study that we turn to now. Adolphs et al. (2004) 
1263 functions as a bridge between the papers already presented, and the later, corpus-

1264 driven studies that follow. Adolphs et al. (2004) echoes some of the concerns of the

1265 earlier papers regarding the status of an applied linguistic researcher in the field of

1266 healthcare, but also promotes the value of corpus-driven analysis and the keyword

1267 method for investigating a healthcare corpus.

1268 2.4.2 Adolphs, Brown, Carter, Crawford and Sahota (2004)

1269 Summary

1270 In this multi-authored paper, Adolphs et al. (2004) sought to establish the role of

1271 corpus linguistics and data driven learning as a means to better understanding the

1272 language of healthcare and healthcare interactions. The authors propose a convergence

1273 of various approaches from the fields of health and social science with those from

1274 applied linguistics, namely conversational and discourse analysis and corpus

1275 linguistics, referring to this new convergence as `a kind of applied clinical linguistics`

1276 (p. 25). The primary motivation for this was to bring new insights and discoveries that

1277 would benefit medical practitioners and patients. The authors' view is that if language

1278 is both interpersonal and transactional, then it becomes vital to understand how the

1279 language in a medical encounter is tailored to the recipient.

1280 The paper presents an analysis of a small corpus of spoken interaction between

1281 NHS helpline staff and callers (researchers playing at being patients), to show what

1282 kind of information can be revealed, and why that information might be significant

1283 within the context of healthcare. Using a keyword analysis to begin with (for an

1284 explanation of this approach, see chapter 3), items of interest were categorised into the

1285 following groups: negatives, imperatives, pronouns, vague language,

1286 affirmations/positive backchannels, directives. Medical terminology was ignored.

1287 These linguistic features were then investigated in greater detail within the corpus and

1288 examined to see where and when they were most frequent. This investigation

1289 established a link between certain linguistic features and particular phases of the

1290 consultation and highlighted what the authors describe as `an overarching tendency

1291 for the health professionals to use politeness strategies and the language of

1292 convergence when speaking with the callers. This was often seen as a downplaying of

1293 the impact of the advice. The results, say the authors, illustrate just how useful an 
1294 exercise uncovering linguistic features of discourse is as it can uncover

1295 communicative patterns that can then be linked to subsequent outcomes, desirable or

1296 otherwise. This is particularly related to issues surrounding compliance and

1297 concordance in healthcare.

\section{Commentary}

1299 Adolphs et al. (2004) makes a convincing case for the need for corpus-based research

1300 into the language of healthcare, research that is both theoretically interesting and that

1301 has a focus on practical outcomes. Drawing on research findings from a good many

1302 non-linguistic areas (the vast majority of references in this paper come from journals

1303 outside of applied linguistics), this was not the first paper to call for a more inclusive

1304 approach to research into the language of healthcare, as Candlin and Candlin (2003)

1305 and Roberts and Sarangi (2003) had done so a year previously, but it was one of the

1306 first to give a clear, practical example of the kind of data that can be uncovered, even

1307 in small corpora, through the application of the keyword method followed by a closer,

1308 qualitative analysis.

1309 The quantitative and qualitative approach, as demonstrated in this study, goes

1310 beyond word frequency and word distribution information and permits access to

1311 subtler, more complex linguistic patterning. The analysis of patient information in this

1312 thesis was undertaken for the same purpose: to uncover the kind of hidden, linguistic

1313 features of the discourse which may have a practical relevance to practitioners and

1314 patients. It is important, too, that Adolphs et al. (2004) were interested in a range of

1315 linguistic features but not in medical terminology/vocabulary, showing that general

1316 vocabulary is as useful an area of study in medical communication as is

1317 medical/technical vocabulary.

1318 Small, in comparative terms, at 35,014 words for the health professionals'

1319 contributions, the corpus used in Adolphs et al. (2004) was sufficiently specialised and

1320 coherent for the size not to be of much concern. It was, say the authors, a 'preliminary

1321 vignette' into the entire NHS Direct corpus and served as a means to show what can

1322 be achieved using the techniques described. Concerns relating to corpus size are

1323 considerably less relevant if the corpus is specialised and targeted and as Biber (1990, 
1324 p269) said, 'descriptive linguistics should not be intimidated by the 'need' for larger 1325 corpora`.

1326 The findings of the study show that health professionals use a range of 1327 politeness strategies and are highly likely to mitigate the advice they give to callers, 1328 even when quoting an authoritative voice such as the British Medical Association, 1329 who were almost certainly more categorical than the health professional referencing 1330 them. The findings also reveal a high frequency of modal verbs can and may which is 1331 significant for this doctoral thesis. Can and may are also the most commonly used 1332 modal verbs in patient information, which, along with mitigators such as just are used 1333 to reduce the threat of the advice or instruction; can and may offer the advice as an 1334 alternative that the patient is free to follow - or to ignore. As I discuss in Chapter 6, 1335 and in the context of my study into the use of deontic modal verbs (those used for 1336 instruction and obligation), this linguistic behaviour seems to be particular to 1337 healthcare advice and not to consumer or legal advice where we find considerably less 1338 mitigation.

1339 Adolphs et al. (2004) is one of the earliest studies to show the utility of using 1340 corpus techniques, particularly a keyword analysis, in revealing important aspects of 1341 spoken medical interactions that may otherwise have remained unnoticed. The paper 1342 was published in the Journal of Applied Linguistics and Professional Practice, a 1343 journal which aims to advance the interdisciplinary nature of applied linguistics

1344 research and which had been launched in the same year as Adolphs et al. (2004). The 1345 paper's authors came from three different disciplines: applied linguistics, health 1346 communication and medicine. The next paper I discuss, Seale, Ziebland and Charteris1347 Black (2006) is also a good example of interdisciplinary research, with authors from 1348 the fields of medical sociology and applied linguistics. The study, published in Social 1349 Science \& Medicine, and which utilises the keyword technique, is an analysis of the 1350 impact of gender on the language used to talk about cancer. 


\section{Summary}

1355 In this study, Seale, Ziebland and Charteris-Black (2006) use a keyword analysis to

1356 investigate the impact of gender on patients' experience of cancer. The study involved

1357 a keyword analysis of forum postings relating to prostate and breast cancer, and of 97

1358 interviews with people with cancer. After an overview of the literature, the authors

1359 (who are careful throughout the paper to reject the notion that gender behaviour is

1360 fixed), suggest that a summary of the literature thus far of gender difference in

1361 language use, communication preferences and illness behaviour might be that 'men

1362 tend to focus on information and women on emotional support. Women draw on wide

1363 informal social and family networks when ill, whereas men deal with things on their

1364 own more, perhaps with the support of their wives, or in collaboration with doctors'

1365 (p. 2580).

1366 The study is in two parts. The first part compares the results of a keyword 1367 analysis of a corpus of interview data (at 727,100 words and including only the 1368 patients' words) with the results of an analysis of the transcripts using the more 1369 conventional thematic approach that is common to social science. In the second part of 1370 the study, the keyword approach is applied to a corpus of over 12,000 forum postings

1371 by people with cancer (PWC). The corpus contained over 1.6 million words. The 1372 objective was to further validate the keyword approach, and to extend the existing

1373 evidence base of gender differences in the experiences of these two common cancers.

1374 The thematic content analysis of the interviews found men seem to prefer to seek

1375 information, and women prefer to engage in social and emotional support when 1376 online. These findings are not to be considered absolute difference, however, say the 1377 authors. The results of the keyword extraction of the interviews paint a far richer 1378 picture. Semantic categories were devised by examining the keyword in context 1379 (KWIC), and words categorised accordingly. Words that could be associated with two 1380 of the coding categories were entered into both categories and marked as a 'split' 1381 word. All 300 keywords were examined, though words that were used less than 10 1382 times were disregarded. 
Keywords related to three semantic categories of Treatment, Procedures and

1384 Tests, and Symptoms and Side effects were very common in the interviews with men.

138574 keywords fell into these three categories, compared to just 18 for the women

1386 interviewed. There were a number of other categories where the difference was just as

1387 significant. Men used more keywords relating to the treatment, to specialist medical

1388 staff and to medical procedures. Women, on the other hand, were more likely to refer

1389 to themselves and named family members, and, unlike men, they frequently used

1390 superlatives, e.g. lovely, amazing, wonderful. Of particular note in this study are the

1391 differences in the keyword category Feelings. 20 keywords were extracted from the

1392 interviews with women; in contrast, only two words appear in this category from the

1393 men: concerned and embarrassment.

1394 The results of the keyword analysis of the web forums confirmed the

1395 differences found in the analysis of the interviews, but also revealed new differences

1396 not seen in the interviews. The authors conclude that the keyword approach has a

1397 number of significant advantages to the analysis of large datasets, such as internet

1398 postings. It is fast, is independent of the views of the researchers, at least in the

1399 extraction stage, and sensitive to context. The technique has an advantage over

1400 traditional qualitative methods in its capacity to reveal areas of interest to the language

1401 researcher that would otherwise remain unnoticed.

\section{Commentary}

1403 This is a very interesting study, and the first of the studies discussed to be concerned

1404 solely with patients. It is also the first study discussed in this chapter to investigate

1405 internet forums, and the first that relates to gender and language use. This paper

1406 introduces the keyword technique to an audience of social scientists, and as we have

1407 seen, different disciplines necessitate different approaches to the disseminating of

1408 results. This is a study that is satisfying for an applied linguist to read: there is a good

1409 level of detail relating to the methodology, and better examples of the data given than

1410 we saw in Skelton and Hobbs (1999a). Nor is there an overly long discussion of the

1411 statistical measures involved in the keyword analysis, presumably because social

1412 scientists are less interested in this kind of detail. 
1413 Seale et al. (2004) were interested in learning how forum conversations by 1414 women about breast cancer differed from those by men about prostate cancer. The 1415 comparative corpus used was not the standard general corpus, but the other dataset:

1416 each set of forum conversations was used as the reference corpus for the corpus under

1417 investigation, i.e. the corpus of breast cancer conversations had, as a reference corpus,

1418 the conversations about prostate cancer, and vice versa. A reference corpus is usually

1419 a general corpus and is chosen to be representative of general language. Forum

1420 postings from people with cancer are highly specialised, however, and the researchers

1421 were intent on showing how each forum differed from the other. The idea that

1422 specialised language requires a specialised corpus has been expressed by several

1423 researchers: there are several studies that use either a dual reference corpus or a single,

1424 domain specific reference corpus (e.g. Baker, 2004; Goźdź-Roszkowski, 2011 and

1425 Grabowski, 2015). Goźdź-Roszkowski (2011) in justifying his choice not to use a

1426 general corpus says, 'comparing a range of specialized genres ${ }^{1}$ from the same domain

1427 against a general reference corpus would inevitably lead to obtaining finding which

1428 may be highly homogeneous and probably valid for legal language in general but

1429 would not help identify features unique to a particular genre' (p36). The same can be

1430 said for any specialised register under investigation, of course.

1431 In this thesis, I use a specialised corpus of general radiography in my keyword 1432 extraction, in addition to an extraction using the BNC, reasoning that each will give

1433 me different kinds of information relating to the differences between patient

1434 information and general information on the one hand, and general radiography on the 1435 other. This is discussed in more detail in Chapter 3, where I present my corpora and

1436 methodology, and in Chapter 4 in my presentation of the keyword analysis and its 1437 findings.

1438 The corpus of postings in Seale et al. (2006) contained 1.6 million words and, 1439 while we do not know how many postings came from each forum, if we assume they 1440 were fairly evenly split, the size of each corpus was around 800,000 words. Opinions 1441 differ regarding an appropriate size for a corpus should be: given the nature of the

\footnotetext{
${ }^{1}$ Gozdz Rowkowski uses genre as I use register in this thesis.
} 
1442 topic and the number of postings (around 12,000 in total), I believe that 800,000 is a

1443 perfectly acceptable size. The authors make no claim about the generalisability of

1444 their findings and are keen to make clear their belief that the concept of gender is not

1445 fixed, adding that expressions of illness may also be highly individual, varying from

1446 individual to individual. Nonetheless, this study's findings are significant, and make a

1447 substantive contribution to the literature on the use of keyword techniques in the

1448 analysis of medical discourse. The findings related to gender differences in the way

1449 people experience a disease or health condition are important and relevant to this

1450 thesis.

1451 Gender difference in information-seeking behaviour is a topic that is well-

1452 researched (e.g. Bidmon \& Terlutter, 2015; Ek, 2013; Rice, 2006; Rutten, Squiers \&

1453 Hesse, 2006). These studies confirm not only the existence of different information

1454 seeking behaviour, and a difference in the type of information being sought, but also

1455 that women are generally more likely than men to engage in online health information

1456 seeking. As Ek (2013) says 'When it comes to health, women seem to be more

1457 engaged, more involved, more attentive and apparently better-informed decision-

1458 makers.' (p742). Seale et al. (2006) found slightly more men than women reported

1459 using the internet for health information seeking, though I think we can disregard this

1460 as the difference was not significant (38\% to 33\%), not all interviewees were asked

1461 about their internet usage and this was not the theme of their study.

1462 Surprisingly, however, in spite of the studies showing how gender relates to

1463 the frequency of health information-seeking online and the differences in the

1464 information being sought (and wanted), gender is not always considered to be a

1465 determinant of healthcare and is very rarely considered when producing health

1466 information. In an analysis of five internationally recognised health promotion

1467 frameworks, Gelb, Pederson and Greaves (2011) state that 'although gender was at

1468 times mentioned as a determinant of health, gender was never identified and

1469 integrated as a factor critical to successful health promotion.' (p. 445).

1470 There is nothing fixed about the linguistic performance of gender (Seale and

1471 Charteris-Black, 2008), but if men and women tend to look for different information

1472 and focus on different aspects of health, illness and treatment, it seems reasonable to 
1473 consider whether existing patient information, such as that in the corpus used in this

1474 thesis, is appropriate to these different needs. Additionally, given that sex and gender

1475 are rarely considered either as determinants of healthcare or when producing

1476 healthcare information, the question is raised of how inclusion criteria for information

1477 is decided?

1478 We have already seen earlier in this chapter that many of the studies of PILs

1479 and information for chronic conditions find that they fail to deliver what patients say

1480 they want to read. Studies also show that a biomedical discourse predominates in

1481 much patient information. A biomedical discourse relates to the body, the disease,

1482 treatment, medical procedures, symptoms and side effects, precisely the areas that

1483 Seale et al. (2006) find foregrounded in the men's discussion of prostate cancer.

1484 With regards to the field of radiography, we do not know what kind of 1485 discourse predominates in patient information, or whether it contains the kind of

1486 information patients want to read, though we have already seen that patients attending

1487 radiography departments often seem remarkable uninformed regarding radiography

1488 examinations and associated risk. The keyword analysis that I describe in Chapter 4

1489 can contribute to our understanding of the first question, while further studies are

1490 needed in order to answer the second question.

1491 Keyword studies of healthcare discourse have also investigated age as a

1492 variable, and the interaction of age with gender. The latter was the focus of Seale and

1493 Charteris-Black (2008), the next study that will be discussed.

1494 2.4.4. Seale and Charteris-Black (2008)

\section{Summary}

1496 Seale and Charteris-Black (2008) investigated the linguistic expression (as illness

1497 narratives) of the experience of a range of cancers in men and women, across three

1498 age groups, older, mid and young. The corpus of interviews amounted to just over 1

1499 million words, made up of 102 interviews. A keyword analysis using Word Smith

1500 tools (Scott, 2005) was carried out, followed by semantic tagging using WMatrix

1501 (Rayson, 2005). This permitted further analysis of the frequencies of the semantic 
1502 groups. These two analyses informed the choice of keywords that were then

1503 investigated qualitatively in the corpus.

1504 The results showed that older men living with a serious illness viewed the 1505 medical system as an expert system and saw their condition as a problem to be fixed, 1506 alone, and with 'medico-scientific solutions'. (p. 1025). This finding supports that of 1507 previous studies (e.g. Seale, Ziebland \& Charteris-Black, 2006). Older women used 1508 more words related to social networks (including the absence of one), using more 1509 keywords from the categories Pets, Living alone, Death and Religion than men, 1510 though women in the Mid-age group spoke slightly more frequently about death than 1511 either younger women and older women. Death was a key topic for men in the Older 1512 and Mid-age groups, too. Findings also showed that women used considerably more 1513 words related to feelings and self-revelation, whereas men used more words from the 1514 categories Strange/Weird and Worry/Concern. One semantic category, Young Style, related to the discourse of younger 1516 people, which contained a large number of adverbs (e.g. basically) and minimisers 1517 (e.g. just) and differed quite markedly from the other two age groups. Older men in 1518 this study did not use any of the words that appeared in this category. The authors 1519 conclude that older men bring considerable social confidence to their interactions in 1520 the medical system, a confidence that young men do not yet possess. In contrast to the 1521 literature that finds older men increasingly dependent on their wives and lacking in

1522 social support, Seale and Charteris-Black (2008) suggests that older men wield a 1523 social power and confidence that does not require the maintenance of strong social 1524 networks.

\section{Commentary}

1526 This keyword study raises some very interesting questions regarding the role of 1527 information in the experience of illness. One particularly relevant finding in the study, 1528 which will be picked up again in Chapter 4, relates to the differences in the naming of 1529 healthcare professionals in men and women's illness narratives. Seale and Charteris1530 Black (2008) found that men, in general, were significantly more likely to refer to 1531 specialists, general practitioners and consultants than were women. Older men in 1532 particular were more likely to mention these professionals as well as other named 
1533 medical specialities. Doctor was used by all subjects more or less equally, suggesting

1534 that men, and older men especially, give particular importance to the specialist

1535 knowledge of the medical professionals they interact with. Nurse, in contrast, was

1536 referred to significantly more often by women.

1537 The study's findings also have implications for our understanding of young 1538 people's experience of serious illness, suggesting that young men in particular lack the 1539 social power and confidence that their fathers and grandfathers possess, and, as a 1540 result, are unable to talk about their illness in the same way. The category Young 1541 Style contained the following words, the vast majority adverbs: basically, obviously, 1542 actually, probably, really, gonna, stuff, yeah, yeh, like, sort of, to be honest, you know, 1543 just, kind of. It is of particular note that young men used these words much more than 1544 young women, and the difference was statistically significant. Many of these adverbs 1545 in speech are associated with indirect strategies that reduce the illocutionary force of

1546 face- threatening speech acts (Brown and Levinson 1987). Even those adverbs that 1547 initially appear to be used as intensifiers - basically, obviously - were found in the text 1548 to be used by young men to minimise the threat of something that was potentially 1549 frightening and very serious:

um well I was diagnosed when I was just about 15 -and-a-halfum with leukaemia which we later found out I had the Philadelphia chromosome as well which can't be treated just by uh radiotherapy it has to, you have to have a bone marrow transplant basically (YPC10 male, aged 19 years). (Seale and Charteris-Black, 2008, p. 1033)

1560 cancer. It also underscores the importance of targeted healthcare information and

1561 healthcare advice forums for young people. Indeed, healthcare advice forums for

1562 young people have been investigated in several key corpus studies of healthcare

1563 discourse, some of which I shall now present. 
1565 Harvey at al. (2008) was one of the first studies to look at young people's e-

1566 communication about healthcare, with the aim of investigating the communication

1567 difficulties experienced by young people when discussing their health concerns. A

1568 corpus of around 1 million words was used, made up of over 62,000 messages from

1569 young people to Teenage Health Freak, a doctor mediated, health advice website,

1570 (which, at time of writing, appears to be offline). The messages were collected over a

1571 period of 24 months. The corpus was analysed using the keyword approach, an

1572 approach common to several of the studies already presented in this literature review.

1573 The comparative corpus used for the keyword extraction was the general spoken part

1574 of the British National Corpus (BNC), resulting in 1160 keywords. Keywords that

1575 related to communication, verbal interaction, and to advice and information seeking

1576 were highlighted, and a collocational analysis was carried out on these words. This

1577 involved the calculation of the Mutual Information (MI) score of the common

1578 collocates of the selected keywords. MI is a measure of the strength of association

1579 between words, and for general language, is considered very reliable. The third and

1580 final stage involved an examination of these keywords and collocations in context.

1581 The results of the analysis were that young people not only use a higher

1582 frequency of words related to communication, e.g. tell, say, talk, answer, but that

1583 these words were overwhelmingly used with negative collocates, Adjectives afraid,

1584 scared, worried, embarrassed and stressed were very common collocates of the

1585 communication verbs. A second finding revealed that the young people commonly

1586 experienced difficulties in communicating their concerns to doctors and family

1587 members, with one reason being the fear that doctors would not respect

1588 confidentiality. A general ignorance of the medical system and the roles of healthcare

1589 professionals were also revealed by the keyword analysis.

\section{Commentary}

1591 This study was the first to investigate adolescent health communication concerns

1592 using corpus linguistic techniques. Authenticity was retained, too, as the messages

1593 (referred to as emails in the study) from the young people had not been edited by the 
1594 website and thus the original wording and the nuances of individual expression were 1595 retained.

The authors are keen to point out that theirs was not an 'outsider' perspective, 1597 a remark that references the concerns voiced in both Candlin and Candlin (2003) and 1598 Roberts and Sarangi (2003) regarding the role of the applied linguist when 1599 undertaking research of healthcare discourse. Harvey et al. (2008) did not decide 1600 what issues needed to be researched; the issues were revealed in the adolescents' 1601 communication, and the study was unusual in that it focussed solely on patient1602 initiated action. This is one of the obvious advantages of the keyword technique in 1603 language analysis. There is, of course, researcher-bias in the decision to focus on 1604 certain keywords and not others, or to focus on one semantic area, those related to 1605 communication in this case, but this is not the same as deciding a priori what will be 1606 investigated in the corpus.

1607 The findings of this study were an important contribution to the literature on 1608 adolescent health and have obvious implications for the training of people who work 1609 with adolescents - and not only healthcare professionals. The study revealed great 1610 anxiety on the part of the adolescents about confidentiality, and their fears about their 1611 health concern being disclosed.

The study also contributes to the literature related to e-health, in particular to 1613 that on computer-mediated, doctor-patient communication. In spite of the fact that a 1614 very large percentage of young people had used the internet to access health 1615 information in 2008 (75\% according to Harvey et al. (2008)), and notwithstanding the

1616 fact that the benefits of email consultation were obvious to some health practitioners 1617 (Car \& Sheikh, 2004a; 2004b), Harvey et al. (2008) make reference to the resistance 1618 they perceive from many health professionals to the application of the internet and 1619 email to healthcare, and the slow response to the changing world of medicine that they 1620 feel many medical general practitioners are guilty of.

1621 A decade has passed since this study was published, and a google search for 1622 the terms $e$ health and adolescent result in many information sites, apps and research 1623 studies that relate particularly to depression and mental health issues in young people. 1624 E health has also been joined by $\mathrm{M}$ health: healthcare information, apps and tests on 
1625 the mobile phone or as wearables. In terms of public healthcare, there is evidence that

1626 the e health revolution faces several barriers that slow its progress. A 2015 study by

1627 Currie, Philip and Roberts found that while age-related disparities in internet use had

1628 declined in the UK, and that a number of e health NHS projects had already been

1629 successful rolled out nationwide, barriers that slow the progress of e health included

1630 capacity issues relating to digital infrastructure, budgets, and technological literacy of 1631 both staff and patients.

1632 Adolescent healthcare, and the importance of understanding the specific

1633 healthcare needs of young people, is a theme taken up by a number of subsequent

1634 corpus linguistic studies, three of which I will overview briefly in the following

1635 section. All three studies involved the Teenage Health Freak website, referred to

1636 above in Harvey at al. (2008). The studies all concern online communication in

1637 healthcare forums, where professional advice is given to young people, and all use the

1638 keyword method.

1639 Harvey, Locher \& Mullany (2013) investigated forum postings in both a UK

1640 and US healthcare advice forum, aimed at young people. The researchers carried out a

1641 keyword analysis and looked specifically at discourse related to HIV/AIDS. The study

1642 painted a rather bleak picture of the effectiveness of public information strategies, as it

1643 found that young people were confused and misinformed about a number of important

1644 aspects, including how the virus is transmitted and the difference between HIV and

1645 AIDS. The results may go some way improving information initiatives directed at

1646 young people,

1647 Weight, eating and body discourses were the themes of a study by Mullany,

1648 Smith, Harvey \& Adolphs (2015). The corpus contained just over 2 million words and

1649 was made up of 113,480 advice requests sent to the site over a 5-year period between

1650 2004-2009. The findings are that weight and eating is a persistent concern among

1651 girls, in particular, between the ages of 11-16. The researchers saw a peak in advice

1652 requests related to weight and eating at the age of 12 , with a second, smaller increase

1653 in advice requests on the topic at the age of 16 . This finding, the data that shows

1654 knowledge gaps at different ages, and the alarming attitude that some young people 
1655 show towards anorexia, which is not always viewed as an illness, has potential

1656 implications for medical professionals and educators.

1657 The final summary in this literature review of keyword studies of adolescent 1658 healthcare interaction is a study by Harvey and Brown (2012), who investigated

1659 adolescents' experiences of self-harm in a corpus of messages taken from the Teenage

1660 Health Freak website between 2004-2008. The study follows the same steps as Harvey

1661 et al. (2008) outlined above: a keyword extraction was performed using the BNC as

1662 comparative corpus, which was followed by semantic categories being assigned to the

1663 keywords. The words specific to self-harm were identified and their collocations

1664 explored in the corpus. Harvey and Brown (2012) find that their analysis reveals

1665 several patterns and commonalities in adolescents' accounts of self-harm, information

1666 which provide important insights for health professionals into what drives rising

1667 numbers of young people to self-harm.

1668 I now conclude the summaries, in this literature review, of studies that have 1669 used a keyword analysis in their investigations of healthcare communication. All the 1670 studies I have presented thus far on the topic have promoted the keyword technique as 1671 a very effective tool in the language researchers kit bag, as evidence that it is: an effective means of identifying the 'incremental effect' (Baker, 2006, p. 13) of patterns across large quantities of text, allowing the researcher and the language learner to discover linguistic routines which are liable to remain submerged in extensive data sets. (Harvey \& Brown, 2012, p. 333).

1677 different corpus techniques in the comparative analysis of four pharmaceutical

1678 registers. Grabowski (2015) is included because it uses a keyword and a lexical bundle

1679 analysis and because pharmacy is sufficiently related to medicine to be deemed

1680 relevant. While there are a handful of lexical bundle studies that relate to medicine

1681 (e.g. Jalali \& Moini, 2014; Jalali, Moini \& Arani, 2015; Samar, Shokrpour \& Nasiri,

1682 2018) they are all related to research papers and thus solely from the area of academic

1683 writing. There have been no published studies of lexical bundles in any other written

1684 medical register. 


\section{Summary}

1687 Grabowski (2015) is an analysis of keywords and lexical bundles from the 1688 pharmaceutical field. A corpus-driven, descriptive study, it used the perspective of 1689 register outlined by Biber (2006), Biber and Conrad (2001; 2009) and others (e.g. 1690 Halliday and Hasan, 1976) to investigate linguistic variation across different registers. 1691 The hypothesis explored in the paper is that language can vary considerably within a 1692 single discourse - in this case pharmaceutical - and this variation relates to the 1693 situational contexts, function and intended users of the texts in question. The great 1694 variation in the types of written discourse in pharmacy (i.e. registers) had previously 1695 been unexplored.

The registers under analysis in this paper were patient information leaflets 1697 (PILs), summaries of product characteristics (SPCs), clinical trial protocols (CTPs) 1698 and chapters from academic textbooks on pharmacology (ATs). The PILs were 1699 accessed from the pre-built PIL Corpus (Bouayad-Agha, 2006), while the other texts 1700 were accessed variously form other pre-built corpora and registers or collected by 1701 Grabowski. The Corpus of English Pharmaceutical Texts (CEPT) with approximately 17022.2 million words was the result. It was this corpus that was also used as the reference 1703 corpus, with the register under investigation removed from it for the analysis.

1704 Grabowski's first focus for analysis was keywords, which was followed by a 1705 second analysis of 4-word lexical bundles. The additional focus on phrase frames 1706 from the 2013 study was dropped in the 2015 paper and appeared as a separate study 1707 in the same year. The results of the analysis showed great variation in the number of 1708 keywords present across the registers, not only in the type of word considered key but 1709 also with the number extracted: academic textbooks having almost 3 times as many 1710 keywords as the clinical trial protocols. The results of the lexical bundle analysis 1711 showed similar variation, finding that while all registers made considerable use of

1712 lexical bundles, CTPs were the most repetitive and formulaic and ATs the least.

1713 Following the taxonomy described in Biber et al. (2004) and Biber (2006), the lexical 1714 bundles were categorised into three types: Referential, Discourse-Organising and 1715 Stance. Referential lexical bundles, while evident across all four text types, were used 
1716 least frequently in PILs. On the other hand, stance bundles - more typically found in

1717 spoken discourse - dominated PILs while being entirely absent from the 50 most

1718 frequent bundles in CTPs.

\section{Commentary}

1720 Grabowski's (2015) investigation of four pharmaceutical registers was an important

1721 contribution to literature on register analysis, notwithstanding some methodological

1722 weakness. It is the only study to date to compare some of the different registers within

1723 the domain of pharmacy, drawing the link between the use of keywords and of lexical

1724 bundles in specialised discourse with their communicative purpose. This was also the

1725 first study to undertake a corpus-driven analysis of keywords and lexical bundles in

1726 patient information leaflets. That the lexical characteristics of patient information,

1727 beyond considerations of complexity, might contribute to its function (and to its

1728 readability and effectiveness) has rarely been the subject of much investigation. A

1729 similar situation exists with procedural medical information such as the patient

1730 information for radiography analysed in this study.

1731 A key finding is that stance bundles predominate in PILs. There were

1732 examples of epistemic stance in the use of words like sure, probable, may, as well as

1733 deontic stance, relating to obligation and instruction, and desire bundles. This result is

1734 surprising, as stance has been found to be more a feature of spoken discourse than

1735 written (Biber, 2006, pp. 157-160); and was also seen to be frequent in patient-

1736 provider interaction (i.e. may) (e.g. Adolphs et al., 2004). Grabowski concludes that

1737 the use of stance bundles, along with the use of advisory keywords, is related to the

1738 function of the leaflets, namely to instruct and advise patients regarding their

1739 medicine.

1740 Grabowski (2015) chose not to use a table to present the categories of the

1741 keywords extracted in the different registers. A table would have improved the

1742 readability of the study, I feel. Keyword findings are reported in writing only which

1743 makes for a lot of dense text. With regard to the lexical bundle analysis, there is also a

1744 lack of precision in deciding what can be categorised as 4-word lexical bundles.

1745 Grabowski (2015) makes reference to a phrase If-clause + ask your doctor or

1746 pharmacist when referring to the PIL corpus. This is clearly not a 4-word lexical 
1747 bundle but something else entirely. There are a number of examples, too, of phrases

1748 that contain primarily content words and/or are complete, e.g. at the desired level and

1749 special precautions for storage. Lexical bundles are usually transparent in meaning,

1750 they tend to be structurally incomplete, and often bridge two structural units, i.e. a

1751 clause or phrase, (Biber and Barbieri, 2007, p8.) Some of the 4-word-bundles in this

1752 study are not, in my view, 4-word lexical bundles Nonetheless, Grabowski (2015) is a

1753 relevant study that provides further evidence of the existence of register variation

1754 within domains of language (e.g. pharmacy, law and medicine) and provides further

1755 evidence of the relationship between the communicative purpose of the text and its

1756 situational function and its keywords and lexical bundles.

1757 Given the primary importance of healthcare in society and the drive to improve

1758 the patient experience, further studies of the lexical characteristics of these registers

1759 are long overdue. The register analysis studies described in this thesis provide insights

1760 into the register of procedural patient information, and differences worthy of

1761 investigation are likely to exist in other sub-registers of medical patient information

1762 such as information produced for sufferers of chronic or terminal conditions and that

1763 produced by charities or non-profit associations.

1764 I now turn to a brief presentation of a book-length work that investigated

1765 modal verbs in medical writing.

1766 2.4.6 Vihla (1999)

\section{Summary}

1768 This book length work, which came out of a doctoral thesis, was an analysis of

1769 epistemic and deontic modal verb use in a number of medical registers. Vihla, who

1770 trained as doctor, compiled a 400,000-word corpus of American medical writing,

1771 which included handbooks and clinical textbooks, research papers, scientific

1772 textbooks, editorials and consumer health articles. These registers - Vihla refers to

1773 them as genres - are categorised into three types: Directive, Argumentative and

1774 Expository, according to their communicative functions. The different communicative

1775 functions predict, says Vihla, a difference in distribution and frequency of modal

1776 verbs. This position is in line with applied linguists (e.g. Biber) who take a register 
1777 analysis perspective to the study of language. Her analysis finds that the highest

1778 frequency of both epistemic (especially may and might) and deontic modal auxiliary

1779 verbs (must and should) are found in directive texts, which include clinical textbooks

1780 and manuals and what are referred to as guidebooks, the closest thing to patient

1781 information in her corpus. These findings suggested that the pattern of use of modal

1782 verbs and their frequency in medical writing does not follow that seen in general

1783 discourse, where will, can, could and would are reportedly most frequent. (e.g. Biber

1784 et al., 2002)

\section{Commentary}

1786 Vihla (1999) is an unusual book for two reasons: its focus is a range of medical

1787 registers, rather than a single register (usually the research paper, as we have seen),

1788 and unusual because it used corpus techniques to investigate a variety of modal verbs,

1789 including deontic modals, when it is often epistemic verbs that get most of the

1790 attention. Only American writing was included, with the justification that American

1791 medical books and journals had (and continue to have) a wide, international

1792 distribution. The texts were complete texts of varying lengths though no mention is

1793 made of how different text length may affect frequency results.

1794 Vihla did not include any semi-modals, however, and her deontic modals are 1795 restricted to should and must. Notably absent from her corpus, too, were patient

1796 information leaflets. While there were certainly leaflets in 1999, they were certainly

1797 less common than they are today and, perhaps, their utility had yet to be appreciated.

1798 The finding that may is the most common modal verb overall is interesting, as is the

1799 finding that should and must are most common in professional directive discourse and 1800 also fairly common in the popular guidebooks.

1801 The limitations to the study are that only two core modals were investigated.

1802 Investigating a range of deontic modal verbs, including semi-modals, in patient 1803 information would answer the question of whether more informal semi-modals, such 1804 as have to or need to, are also frequent in this kind of medical writing.

1805 It is to the topic of patient information specifically, and to the third and final 1806 section of this literature review that we now turn. 
1808 The following two studies call for a different approach to healthcare materials, both in 1809 their development and their assessment. These studies highlight the unsuitability of

1810 traditional readability measures and make the case for a new approach that

1811 incorporates knowledge from the field of Systemic Functional Linguistics, in the case

1812 of 2.5.1, Clerehan, Buchbinder and Moodie (2005), and in the case of 2.5.2,

1813 Zarcadoolas (2011), knowledge from pragmatics and text linguistics.

1814 2.5.1 Clerehan, Buchbinder and Moody (2005)

\section{Summary}

1816 Clerehan and colleagues developed and applied a linguistic framework to assess the

1817 quality of medical information leaflets, in this case for the drug methotrexate used to

1818 treat rheumatoid arthritis. This was the first time such an analysis had been carried

1819 out. The linguistic framework is based on Systemic Functional Linguistics (SFL)

1820 (Halliday, 1994), a theory that considers language to be 'a pattern of interlocking

1821 systems, from the smallest unit (e.g. words or phrases) up to the largest (e.g. a

1822 paragraph or a longer piece of text)' (Clerehan et al. 2005, citing Halliday, 1994). In

1823 SFL, meaning is constructed by the reader via the interaction of text (in written

1824 discourse) and its context, which is key.

1825 Clerehan et al. (2005) take the view that typical readability assessments, which

1826 use statistical averages of sentence and word length, are an inappropriate tool for

1827 evaluating patient information materials and do not consider the very many skills

1828 needed by a reader of healthcare materials, or the role the organisation of information

1829 has in comprehension. An assessment framework containing nine communicative

1830 elements was developed, based on the theories of SLF and the concepts of health

1831 literacy referred to above. These elements were then used to investigate 18 leaflets of

1832 varying length, ranging from half-a-page through to a six-page folded brochure.

1833 Words/token data was not given.

1834 Clerehan et al. (2005) found that while all the drug information leaflets 1835 possessed a generic structure, only two of the moves outlined in the framework were 
1836 obligatory (dosage and side-effects). In Clerehan and Buchbinder (2006), which is a

1837 different write-up of the same study, this claim is hedged by the addition of 'possibly'.

1838 Inclusion of the other moves appears optional, while all of the other items evaluated

1839 showed variability across the texts. Evaluating patient information with a linguistic

1840 tool is, say Clerehan et al. (2005), is a far more successful tool towards consistency of

1841 the register than commonly-used statistical analyses (i.e. readability measures). The

1842 authors proposed further work to evaluate the utility of the framework for writers of

1843 patient communication (Clerehan, Hirsh \& Buchbinder, 2009) and to develop and test

1844 a protocol based upon the framework for reader-focussed evaluation. (Hirsh,

1845 Clerehan, Staples, Osborne \& Buchbinder, 2009)

\section{Commentary}

1847 Clerehan et al. (2005) and Clerehan and Buchbinder (2006), with a longer, more

1848 considered conclusion and more methodological detail, proposed for the first time a

1849 linguistic and communicative approach to the development and evaluation of patient

1850 information materials.

1851 While Clerehan and colleagues took the view that all bar one of their elements

1852 were linguistic in nature, it may be more accurate to say that all elements were

1853 communicative in nature, in that they contributed to the communicative success of the

1854 leaflet. Five elements related to the physical organisation of the text, to its appearance,

1855 including visual content and layout, and validity (e.g. accuracy) of the information.

1856 The remaining four elements related to the language used by the writer and were thus

1857 linguistic: the technicality of the vocabulary; the role relationships in the text,

1858 demonstrated through either the use of pronouns or nouns for the patients and medical

1859 professionals (i.e. the patient or you); meta discourse, i.e. the language about the text,

1860 and rhetorical elements, i.e. the linguistic function of each move.

1861 In the 2006 study, Clerehan and Buchbinder give a little more methodological

1862 detail and organised the elements above into what they refer to as levels of analysis:

1863 The first is discourse semantics, under which we find the technicality of lexis (also

1864 described as specialization), role relations, and organisational aspects (including

1865 visuals and headings); the second level of analysis is lexicogrammar, under which

1866 mood and theme is grouped. The theme, say the authors, is generally found in the 
initial part of a clause and contains known or familiar information. Mood, as described

1868 by Clerehan and Buchbinder (2006) is what is usually referred to as stance in the 1869 literature.

1870 Some of the findings are of direct interest to this study. In both the 2005 and

1871 the 2006 studies, the authors go beyond the concept of technicality of vocabulary to

1872 consider other sources of lexical confusion, such as parsing sentences where terms are

1873 presented as synonyms with the addition of the word or, e.g. 'Methotrexate may

1874 cause a reduction in the number of white cells or platelets in the blood'. This can be

1875 doubly-confusing for patients if they are unfamiliar with either or both terms as they

1876 do not know if the second word is a synonym or a new, additional word.

Another area of relevance in Clerehan and Buchbinder (2006) is the reference

1878 to the blurring of 'shouldness', (the authors cite Iedema (2007)), where the language

1879 of obligation, probability and suggestion are mixed, e.g. taking more than the

1880 prescribed dose could be dangerous', increasing, the authors say, the likelihood of

1881 patient confusion. The analysis conducted for this doctoral thesis on modal verbs,

1882 reported in Chapter 6, will reveal whether the blurring of 'shouldness' is also an issue

1883 in procedural information or whether obligations and instructions are presented 1884 directly.

We have seen already in this literature review the problems associated with

1886 frequency markers and patients' over-estimation of risk. The use of frequency

1887 markers, very common in drug information, also receives attention from Clerehan and

1888 colleagues. Seldom, rare, sometimes and usually are often used in patient information

1889 without quantifying more precisely, say the authors. We have already seen, however,

1890 that neither quantifiers or a combined approach to the presentation of risk information

1891 is without problems. Clearly this is an area that requires more investigation.

1892 In the Clinical Contact move, Clerehan et al. (2005) find a variety of linguistic 1893 structures, including imperatives. These structures, they conclude, are either offering a 1894 service (please consult your doctor) or instructing (inform your doctor) (p339). The 1895 addition of please does not always make an imperative an offer, however. This 1896 observation was acknowledged in the revised paper, Clerehan and Buchbinder (2006). 1897 Here, the example of an offer, please see your doctor, from the 2005 study (which is 
1898 an imperative and not an offer), is replaced by please do not hesitate to contact me, 1899 which clearly invites (e.g. offers) contact.

1900 Neither study summarised above was a corpus study - just 18 documents were

1901 selected from 91 about rheumatism (as we learn in the 2006 study, though this

1902 information is absent in the 2005 paper): 15 produced by individuals/hospitals; one

1903 from the Arthritis Association (of Australia), one from a medical journal and one from

1904 a pharmaceutical company. All documents relate to the same treatment drug but in

1905 some documents the intended recipient is not a patient but more likely a doctor.

1906 Clerehan and Buchbinder (2006) suggest that this may mean that the role relationships

1907 can be unclear and status relations can vary within a document, leading to patient

1908 confusion. My view is that it would have been better to exclude these documents from

1909 the analysis as it is quite possible that many more of the elements analysed will differ

1910 when the recipient is a professional. not just role relationships and status relations. As

1911 the documents were all different lengths and produced by different people (in many

1912 cases unknown), controlling for recipient and topic would provide more consistent

1913 information.

1914 The lack of methodological detail in the 2005 study was, to a certain degree,

1915 rectified in the 2006 study, though we still do not know how the 'technicality' of

1916 vocabulary was decided, or whether verbs were chosen to mark rhetoric function and

1917 if so, how were they decided upon and counted. The size of the study also limits its

1918 applicability but, nevertheless, the linguistic focus taken in both papers is ground-

1919 breaking, going far beyond the usual reference to everyday terms or simple language

1920 that many studies of the utility and comprehensibility of patient information leaflets

1921 refer to. A subsequent study (Clerehan, Hirsh and Buchbinder, 2009) applied the

1922 framework to a new set of leaflets, showing the usefulness of the framework.

1923 What is surprising, however, is that the impact of these studies have not been

1924 felt more strongly: more than a decade on, standard readability indexes, e.g. Flesch-

1925 Kincaid and Flesch Reading Ease, continue to be used in studies that evaluate the

1926 comprehensibility and utility of patient information (e.g. Lampert et al., 2016; Paz et

1927 al., 2017) and there are still very few studies that consider or investigate the linguistic

1928 and communicative features of patient information. One important study that does 
1929 consider the linguistic features of patient information is Zarcadoolas (2011), who

1930 discusses the usefulness of pragmatics and text theory in healthcare message

1931 simplification. It is the final study presented in this literature review.

$1932 \quad$ 2.5.2 Zarcadoolas (2011)

\section{Summary}

1934 The problem of the low health literacy of millions of Americans - which is referred to

1935 as the 'silent killer' - is the motivation for the call presented in this paper for a new

1936 approach to the production of health materials for the $21^{\text {st }}$ century. Zarcadoolas (2011)

1937 questions the role and efficacy of text simplification, particularly as healthcare

1938 materials become increasingly complex, and our understanding grows of the

1939 multifaceted nature of health literacy. A discussion of the utility of applying two

1940 models from sociolinguistics - pragmatics and text theory - to develop a 'richer, more

1941 theory-based understanding of text structures and function' (p. 338) is presented.

1942 Simplified text and readability measures - which we have already seen are

1943 considered by some researchers to be poor tools in the assessment of healthcare

1944 materials - seem an inadequate response to the complexity of healthcare information

1945 and the multifaceted, complex nature of health literacy, with its social, cultural and

1946 environmental links. Simplified text can affect coherence, and yet we know, too, that

1947 cohesion and content are vital in order to decode meaning. When text is overly

1948 simplified, information can be missing, and the natural inference that takes places

1949 when we read is affected, says Zarcadoolas (2011), adding that most meaning takes

1950 place in 'beyond-the-sentence chunks in the text and the interaction between the text

1951 and the reader/listener (pragmatics)' (p. 342). Unwittingly, says Zarcadoolas (2011),

1952 simplified messaging may be making it more difficult for individuals to get the

1953 information they need to make informed healthcare decisions.

1954 A 'health literacy load analysis' (Zarcadoolas and Pleasant, 2008) is presented

1955 as a possible solution to the problems described above. The aim of the analysis is to

1956 assess the difficulty of a text by using linguistic text models in conjunction with an

1957 ecological model of health literacy. The analysis 'unpacks' a text and requires an

1958 identification of the aspects that are likely to affect comprehension at the surface- 
1959 level, text level and at the pragmatic level. The author concludes with a partial load

1960 analysis example and an acknowledgement of the need for further research into their

1961 proposed health literacy load analysis.

\section{Commentary}

1963 Zarcadoolas (2011) is one of very few studies to propose that knowledge from the 1964 field of applied linguistics - specifically pragmatics and text theory - be applied to the 1965 evaluation of healthcare materials. Another researcher who also calls for applied 1966 linguistic involvement in this area is Rubin (2014), who states that 'the symbiosis 1967 between health literacy and applied linguistics is strong'. Both are pragmatic 1968 disciplines, Rubin says, adding that perhaps the greatest contribution that applied 1969 linguistics can make to health literacy is to develop 'modality and context-sensitive 1970 parameters for characterizing and then mitigating health message complexity'. (p. 1971 161).

1972 Zarcadoolas and Pleasant's (2008) health literacy load analysis may be useful 1973 in this regard. Along with the knowledge of how we process meaning, and the role of 1974 cohesion and content in making meaning, Zarcadoolas and Pleasant (2008) present 1975 other literacies they say are required to comprehend many healthcare messages: basic 1976 functional literacy; science literacy, which relates to basic scientific and technical 1977 knowledge; civic literacy, which relate to media literacy, the capacity to assess the 1978 source of the information, knowledge of governmental and civic systems, and 1979 awareness of personal responsibility; and finally, cultural literacy.

The framework developed by Clerehan et al. (2005) seems more helpful for 1981 the developers of healthcare leaflets perhaps, as it focusses clearly on the 1982 communicative and linguistic aspects of the leaflets, permitting a step-by-step 1983 approach to both development and evaluation. Zarcadoolas (2011) refers to the need 1984 to 'unpack' the text at the surface-level, but there is no detail given, and only a partial 1985 example of a health literacy load analysis is provided. The surface-level of the text I 1986 take to mean the structure of the text and the lexis used, though 'unpacking' is a vague 1987 term if the writer of the text fails to see how the words they choose, or the way in 1988 which they express certain ideas, can be problematic for the reader. Zarcadoolas 1989 (2011) seems to relate more to the application of applied linguistics knowledge to the 
1990 definition of health literacy, than it does to the production and evaluation of healthcare 1991 materials.

1992 Nonetheless, Clerehan et al. (2005) and Zarcadoolas (2011) are significant 1993 contributions to the very small, but steadily growing, body of literature that sees the 1994 value of applied linguistic approaches to the study, development and appraisal of 1995 healthcare materials. My own research, I hope, will also be considered a useful 1996 contribution to the literature.

1997 2.6 Conclusion

1998 In this chapter I have presented a literature review of key studies relating to a variety 1999 of academic medical registers, including research papers and case notes. I have 2000 included, too, an overview of the literature relating to patient information leaflets for 2001 pharmaceutical products, commonly known as PILs, and consent forms, suggesting 2002 that the principal themes common to these studies may also be common to procedural 2003 patient information. These themes include readability and how risk is expressed and 2004 understood.

2005 The second part of my literature review began with a presentation of two 2006 studies that highlight the challenges and the importance of real-world outcomes of 2007 language research into the discourse of healthcare and healthcare materials and 2008 concludes with two studies that underline the need to approach the development and 2009 evaluation of these materials in a new way. These studies highlight the importance of 2010 text cohesion in the comprehension of the materials, underlining the difference 2011 between literacy and health literacy and emphasising the insights that linguistic 2012 research can bring to the discussion.

2013 The importance of coherence and cohesion in text comprehension is referred to 2014 by Zarcadoolas $(2011 ; 2013)$ and Clerehan et al. (2005) though it is an area that, to 2015 date, has not been sufficiently investigated in relation to healthcare materials.

2016 Simplifiied text, which is the standard approach to the production of healthcare 2017 materials, may even be at odds with the concept of cohesion as 'text that is highly 2018 cohesive maintains continuity of ideas. If there are few or no connections between 2019 ideas/sentences in a given text, readers need to bridge the cohesion gap through 
inferences (Singer \& Ritchot, 1996). As Liu and Rawl (2012) and Zarcadoolas (2011)

2021 say, health literacy issues can mean that patients are unable to bridge the cohesion gap

2022 through inferences as they have none to draw on. This is likely to be the case when the

2023 healthcare information pertains to rare or unusual conditions, or to conditions that are

2024 not commonly discussed, but whether a health topic is commonly discussed will vary

2025 from one individual to another and is dependant on social, cultural and religious

2026 appropriacy. Even relatively common conditions may be entirely unfamiliar to some

2027 people. The same applies to novel experiences, such as a radiographic exam, the focus

2028 of the material investigated in this thesis.

2029 In Liu and Rawl's study (2012), the researchers conclude that higher text

2030 cohesion facilitates the reading speed and comprehension of colorectal screening

2031 information, (but not retention of vocabulary), while a study from Finnish researchers

2032 (Kaakinen, Salonen, Venäläinen, \& Hyönä, 2011) on the relationship between

2033 cohesion and expository text - and healthcare information materials often have an

2034 expository role - found that high cohesion text was not only more persuasive than low

2035 cohesion text, but that attitude after reading predicted successful recall of the message

2036 arguments. In healthcare materials, it is not necessarily the vocabulary that needs to be

2037 remembered but the message: if readers have understood the reasons why not eating

2038 before an operation is important, and this message has been presented persuasively,

2039 perhaps they are more likely to remember and act upon it. Further research in this area

2040 is very much needed.

2041 The lexico-grammatical patterning of a register is linked to its cohesion, and a

2042 lexical bundle analysis reveals this patterning in a register (e.g. Conrad \& Biber, 2005;

2043 Biber \& Barbieri, 2007). There are very few lexical bundle studies of non-academic

2044 medical registers and Grabowski (2015), presented in this chapter, is the only such

2045 study that I am aware of. The gap in the literature is enough of a motivating factor to

2046 conduct such an analysis of procedural patient information. The other motivation,

2047 however, is that such an analysis may well reveal valuable information about the level

2048 of cohesion of the text, with corresponding insights relating to its predicted ease of

2049 comprehension and message retention. The methodology of a lexical bundle analysis

2050 and some key studies will be presented in the next chapter, with the results of the

2051 analysis presented in chapter 5. 
In this chapter I have also presented a number of studies that use the keyword

2053 approach to investigate practitioner and patient language. The keyword approach is

2054 the most utilised corpus technique in the healthcare discourse literature, principally

2055 because it allows a more sensitive uncovering of areas of interest than traditional

2056 qualitative methods. Applying a keyword extraction to patient information for

2057 radiography may reveal some interesting information about its characteristics, both

2058 semantic and thematic, and allow, perhaps, a comparison between this register and

2059 what we know of other medical registers, as revealed by existing studies, some of

2060 which I have included in this chapter. The papers presented in this chapter are

2061 keyword studies of practitioner spoken language (Adolphs et al., 2004 and Skelton

2062 and Hobbs, 2009a) and the written and spoken language of the patient. In my study, I

2063 apply the approach to official written language of the healthcare provider, procedural

2064 patient information. The methodology of the approach is presented in chapter 3 while

2065 the results of the analysis are presented in chapter 4.

2066 Vihla (1999) provides the springboard into my analysis of modal verbs for

2067 instructions, which I present in chapter 6. Patient information was notable by its

2068 absence in her research with a likely explanation being that in the age before universal

2069 access to the internet, patient information was neither produced or digested at the rate

2070 it is today. My analysis also extends the range of modal verbs under analysis to

2071 include the semi-modals have to and need to, in addition to should and must, the two

2072 modals Vihla investigated in her corpus. The 'blurring of shouldness' referred to by

2073 Clerehan et al. (2005), citing Iedema (2007), where the language of obligation,

2074 probability and suggestion are mixed, e.g. taking more than the prescribed dose could

2075 be dangerous' leading to possible comprehension issues, is also of interest. By

2076 extracting the modals and semi-modals used for instruction in the corpus, and

2077 examining their uses in the patient information, we can have a clearer idea of how

2078 they may help or hinder the comprehension.

2079 We now turn to the detailed methodology of the corpus approaches I have

2080 referred to, preceded by a presentation of the corpora the software that was used for 2081 the analyses. 
2083 We have seen in chapter 2 just how useful the keyword extraction is for uncovering

2084 hidden discourses, beliefs and attitudes. Applying the keyword technique to patient

2085 information for radiography is likely to reveal some interesting findings about the

2086 register. A lexical bundle analysis, on the other hand, has not been applied to

2087 healthcare materials, though the literature shows it can reveal important information

2088 about the communicative function of a register. Such an analysis can provide insights

2089 into the structure of patient information for radiography and the structure of a text is

2090 an important factor in how complex it is or is perceived to be, as we have seen in the

2091 previous chapter. The readability of patient information is an ongoing concern, but the

2092 inadequacy of readability measures for the evaluation of patient information is also an

2093 issue. A lexical bundles analysis may well contribute to our understanding of the

2094 underlying structure of the patient information and from this, we may have a better

2095 idea of how its cohesion relates to its readability. The third analysis carried out as part

2096 of this doctoral thesis is on the use of modal verbs to express obligations and to give

2097 instructions. Given that instructing is one of the primary functions of patient

2098 information, it is important that we evaluate the lexical means used to express these

2099 instructions. The concepts of adherence and compliance appear differently if patients

2100 have not understood that an utterance was intended to instruct, and thus did not realise

2101 they were expected to do something. The 'blurring of shouldness' that Clerehan et al (

2102 2005) refer to may well cause comprehension problems, as referred to in chapter 2 ,

2103 but this idea has not yet been explored in healthcare materials.

2104 This chapter presents the methodologies and techniques used in this study,

2105 beginning with a description of the patient information corpus, the material it contains

2106 and how this material was selected. This description is followed by a similar

2107 description of the two other corpora that I built for the purposes of comparison: a

2108 consumer information corpus and a general radiography corpus. After a detailed

2109 description of the steps taken to construct the corpora, I present the software that was

2110 used: Sketch Engine. This, in turn, is followed by a detailed presentation of the

2111 methodology of the keyword and lexical bundle approaches, along with the 
2112 methodology used for the third analysis of deontic modal verbs, those modals that are

2113 used for instructions and obligation.

\section{$2114 \quad 3.1$ The Patient Information Corpus}

2115 The patient information that was used in this research was procedural patient

2116 information for radiography. As I state in the preceding chapter, we can think of this

2117 as a sub-register of patient information. This kind of information is given to people

2118 who are attending hospital or medical centre for a diagnostic radiographic

2119 examination and given to patients who are undergoing a medical operation or

2120 intervention that involves the use of a radiographic technology, such as angiography

2121 or bronchoscopy. Patient information is also written for patients who are undergoing

2122 radiation therapy for cancer.

2123 To put the topic of radiography into some context, it is helpful to know which

2124 exams are commonly carried out, how frequently they are performed and which use

2125 radiation. Table 1 presents a snapshot of imaging activity in the NHS over a 13-month

2126 period. The radiographic examinations most frequently performed by the NHS are x-

2127 ray, computed tomography (CT), magnetic resonance imaging (MRI) and ultrasound.

2128 The last, ultrasound, is also referred to as sonography or ultrasonography. CT and x-

2129 ray use ionising radiation. Table 2 gives a snapshot of the numbers and type of

2130 radiographic exams performed between January 2016 and January 2017 in the UK by

2131 the NHS.

2132 Table 2 Count of imaging activity in England, on NHS Patients, January 2016 to January 2017

\begin{tabular}{lcc}
\hline Modality & Number in 2016 & Radiation used? \\
\hline X-ray & $22,398,045$ & Yes \\
Ultrasound & $9,099,225$ & No \\
CT scan & $4,655,065$ & Yes \\
MRI & $3,234,690$ & No \\
Fluoroscopy & $1,033,250$ & Yes
\end{tabular}


Nuclear Medicine

PET-CT scans

SPECT scans
418,220

125,640

31,015
Yes

Yes

Yes

2133 Note: Data from https://www.england.nhs.uk/statistics/wp-content/uploads/sites/2/2016/08/Provisional-Monthly-

2134 Diagnostic-Imaging-Dataset-Statistics-2017-05-18.pdf

2135 According to government figures (Baker, 2018), the number of CT scans

2136 carried out by the NHS has increased by $43 \%$ in the period 2013-2018, while MRI

2137 scans are up by $42 \%$. Increases such as these have been reported in most countries in

2138 the world (IAEA, 2015). In spite of the frequency reported above, we have seen

2139 already that patients know very little about the examinations being performed in

2140 radiography suites (Singh et al., 2017; Ukkola et al., 2017) and they are even less

2141 knowledgeable about radiation risk (Hansberry et al., 2014; Singh et al., 2017). These

2142 findings, along with the increase in the number of scans being performed annually,

2143 strongly suggests that comprehensible patient information for radiography has never

2144 been more necessary.

\section{$2145 \quad$ 3.1.1 Corpus contents}

2146 The corpus used in the analysis was made up of 221 written patient information

2147 documents for radiographic examinations and medical interventions that involved a

2148 radiographic procedure, such as angiography or bronchoscopy. The corpus was first

2149 compiled in 2011, with later additions in 2014 and 2016. Both diagnostic and

2150 therapeutic procedures were included, though the majority of documents relate to

2151 diagnostic exams and medical procedures involving the use of radiographic

2152 technologies. The patient information documents were all Word or pdf documents that

2153 were available online. The sources of the information were NHS hospitals in the UK

2154 (54 documents), the British Society of Interventional Radiology (37 documents) and a

2155 US radiology patient information website, www.radiologyinfo.com (130 documents),

2156 produced by the Radiologic Society of North America (RSNA). The RSNA is a non-

2157 profit association. 
I chose to include patient information from both the UK and the US as the

2159 internet means that patients are not restricted to information produced in their own

2160 countries and may freely read various documents from a range of countries. Radiology

2161 Info is a very well-known and well-respected information website with, it is reported,

2162 around 700,000 visitors a month. (cited in Hansberry, John, A., John, E., Agarwal,

2163 Gonzales \& Baker, 2014). A search in Google for 'CT information' returns

2164 Radiology Info as the first result. A search in Google for CT information UK' returns

2165 the NHS. With regards to the latter, it was particularly NHS hospital websites that

2166 were the source of the information materials, as it was important that only websites

2167 that offered printable, stand-alone documents were included, as I will now

2168 explain.Websites that offered information but not as a .pdf or a Word document were

2169 not included. Nor were forums or chat rooms. The focus of this thesis is on official

2170 patient information produced by hospitals and medical trusts, or developed by medical

2171 associations with professional authority, producing information for both patients and

2172 professionals. Peer-to-peer online communication, where patients give information

2173 and advice to each other, or general medical websites reporting on radiographic

2174 procedures, were both excluded. As the documents were available as pdf or Word

2175 documents, I assumed that the patient was expected to print off the information

2176 (although whether patients did or did not print off information was irrelevant to the 2177 study.)

2178 The corpus contained 408,997 words and a total of 221 documents. There were

217937 documents produced by the British Society of Interventional Radiology; these were

2180 published between 2010 and 2011. The 54 documents from the NHS hospitals were

2181 published or updated between 2007 and 2015 while the remaining 130 documents

2182 from RadiologyInfo.org were published between 2013 and 2014. 
2187 Table 3 shows the make-up of the corpus.

2188 Table 3 The contents of the corpus of patient information for radiography

\begin{tabular}{lll}
\hline Source & N word & N docs \\
NHS (UK) & 62,957 & 54 \\
British Society of Interventional Radiology (UK) & 51,654 & 37 \\
RadiologyInfo.org (US) & 294,386 & 130 \\
& & \\
& $\mathbf{4 0 8 , 9 9 7}$ & $\mathbf{2 2 1}$ \\
\hline
\end{tabular}

2189 3.1.2 Corpus size

2190 Sinclair (2004), when referring to corpus size, asserts that 'small is not beautiful; it is 2191 simply a limitation' (p. 89), but while the corpus used in this study is small, it is also 2192 specialised. A specialised corpus has been described as a collection of texts delimited 2193 by a particular register, discourse domain, or subject matter (De Beaugrande, 2011).

2194 As a specialised corpus, its size is appropriate, and I included only patient information 2195 that met certain criteria, as described above in 3.1.1. Nor are small, specialised corpus 2196 studies that investigate medical language considered unusual. Indeed, it would be 2197 difficult to find a researcher working in the field of specialised registers who would 2198 argue for the need to use a corpus greater than 1 million + words. The handful of 2199 people who suggested such a thing in the course of my research were not people 2200 working with corpora on a regular basis or even at all.

2201 Specialised corpus studies in the domain of medical language that use small 2202 datasets include Yang et al (2015), who look at epistemic modality in medical 2203 research papers in a corpus of around 80,000 (exact count not given), and Webber 2204 (2005), who considers interactive features in a 34,692-token corpus from medical 2205 conference presentations. Adolphs et al (2014) investigated a 61,981-word sub-corpus 2206 of the Nottingham Health Communication Corpus. The sub-corpus were transcribed 2207 telephone conversations between NHS Direct health advisors (NHS Direct was a 
2208 telephone health advisory service run by the NHS, no-longer active) and patient

2209 callers. As Adolphs et al. (2004, p. 13) say: 'Although these numbers are relatively

2210 small compared to many corpora, the specialised nature of this health care dialogue

2211 made this collection sufficient for an initial, corpus linguistic investigation into the

2212 language data'.

2213 Skelton and colleagues, who were among the first to utilise a combined

2214 quantitative and qualitative approach to medical discourse (e.g. Skelton and Hobbs,

2215 1999a s 1999b; Skelton et al., 1999; Skelton et al., 2002a; 2002b), also used relatively

2216 small datasets - often around 500,000 words (exact totals were not given). While

2217 stressing the importance of quantitative data, Skelton and Hobbs (1999) also underline

2218 the need for context and qualitative information in interpreting the data, saying that

A basic concept in the study of language is that meanings cannot be completely quantified: if words were like numbers, it would be hard to understand why we bother with both. Any quantitative analysis must, therefore, take place in a qualitative context. (p. 109)

2223 Their view and that of many researchers working in the field of healthcare discourse is 2224 that mere frequency counts alone are unlikely to uncover the kind of patterns that are

2225 of interest, making a strong case for smaller corpora and combined approaches to data 2226 analysis.

\section{3.1.3 Document type and variation}

2228 Documents varied considerably in length, from around 300-4388 words. The

2229 information from the US site, RadiologyInfo.org, contained the longest documents:

2230 nearly $60 \%$ of the documents were longer than 2,000 words, with around $25 \%$ longer

2231 than 3,000 words. The site was also responsible for a document on urography at 4388

2232 words. In contrast, just $10 \%$ of the NHS documents and barely $3 \%$ of those from the

2233 Society of Interventional Radiography were longer than 2,000 words.

$2234 \quad 42 \%$ of the documents are from UK sources and $58 \%$ from a US source in the 2235 corpus: in terms of the number of documents, then, the corpus is balanced. However, 2236 the lengths of some of the US-sourced materials means that the ratio of words in the 
corpus is less balanced: 114, 611 words in the UK sourced materials compared to 294,

2238386 in the US sourced, which is a ratio of around 1:2.5.

It is interesting to note that the complexity of the patient information material on

2240 RadiologyInfo.org has been the subject of some criticism, and virtually all of their

2241 material scored poorly when subject to a raft of readability tests (Hansberry et al.,

2242 2014). I have, however, already referred to the shortcomings of readability measures

2243 to evaluate patient information, none of which, to my knowledge, include the length of

2244 the original document in their appraisal. Perhaps all that can be said about the

2245 materials in RadiologyInfo.org is that they are long, which may well put people off

2246 reading them.

2247 The decision to use only UK- and US- sourced material was also taken because 2248 of the difficulty I had in sourcing material that fitted my criteria, and that was readily

2249 available on websites from other English-speaking countries. I had assumed that

2250 patient information is as ubiquitous elsewhere as it is in the UK and the US, or at least

2251 digitally ubiquitous, though it turns out not to be the case. The reasons for this vary,

2252 though technological advancement (i.e. making information available digitally),

2253 budgets (i.e. the cost of producing patient information) and how patient-centred a

2254 healthcare system is (i.e the perceived need for patient information) are likely to be

2255 primary factors. Due to the difficulties of finding suitable examples that fitted my

2256 inclusion criteria (see above), a decision was made to use British and American

2257 English examples only. As I have already mentioned, both RadiologyInfo.org and the

2258 NHS material is the first to be presented in a Google search, so we can be assured that

2259 these sources are likely to be the first that patients from the respective countries look

2260 at when online and searching for information about radiography.

\section{$2261 \quad 3.1 .4$ Document lengths and sampling}

2262 But does the difference in length of the documents in the corpus matter? I believe that 2263 the length of the texts matters far less than the fact that all documents were complete 2264 and, as I explain below, the length of a document may even be a factor that is related 2265 to culture and to concepts of uncertainty avoidance. With regard to corpus building, 2266 Adolphs (2006) recommends that texts making up a corpus should be complete, 2267 though other researchers suggest sampling (McEnery, Xiao \& Tono, 2006), and have 
2268 stressed the need for documents of a similar size when building general corpora.

2269 However, sampling is not something that is necessarily easy or advisable to do, which

2270 I will explain in more detail in 3.1.5. Sampling is not only something that would have

2271 proved difficult to do, giving the enormous variation in length of the documents in the

2272 corpus referred to above, but would not have been a good idea, either, given that the

2273 documents were relatively short and all of the information contained in them, and

2274 where that information occurred, I considered important.

2275 It may also be the case that the length of a document is a possible indication of

2276 cultural differences and thus a factor worth reporting on. As we have seen, patient

2277 information for radiography can be considered a sub-register of patient information,

2278 but it should be remembered that even within this sub-register there are likely to be

2279 differences that future studies could explore. These differences include those that

2280 relate to the variety of English, e.g. US and UK and those that compare English with

2281 other languages. There have been similar studies for the sub-register of the PIL

2282 (Biancho, 2016) with findings that suggest this is a fruitful area for further studies, as

2283 how information is presented and what is prioritised may well be culturally specific.

2284 How risk is perceived, for example, has been shown to be culturally specific

2285 (Gerritsen, Nederstigt \& Orlandi, 2006; Van Berkel and Gerritsen, 2012) and

2286 information pertaining to side-effects in PILs can be presented differently depending

2287 on the language. Van Berkel and Gerritsen (2012) demonstrated this in their study of

2288 side-effect information in a drug leaflet produced for Flanders and the Netherlands,

2289 the latter a low uncertainty avoidance culture where people fear risk less and do not

2290 feel the need to have the details related to risk made clear, the former a high

2291 uncertainty avoidance culture. Five leaflets for an ibuprofen medication were

2292 compared. The leaflet produced for Flanders was presented in three languages: Dutch,

2293 French and German, though it was the Dutch content alone that was compared.

2294 Significant differences were found by Van Berkel and Gerritsen (2012) in the amount

2295 of risk information included and the number of medical terms used, and while the

2296 leaflet from Flanders was, in four cases, considerably longer than the version for the

2297 Netherlands, though the authors did not find the difference in length to be statistically

2298 significant. 
Reducing texts in size by sampling them may remove important information that

2300 relates to the readership and production culture. This kind of information is

2301 increasingly important as new laws come into effect in Europe relating to the

2302 provision of lay clinical trial summaries. These lay summaries must also be translated

2303 into the languages of the countries where pharmaceutical products have been

2304 evaluated. Van Berkel and Gerritsen (2012) shows us that a one-size-all approach is

2305 not appropriate when discussing risk or when using medical terms. In addition to the

2306 reasons presented above, sampling patient information may unwittingly remove key

2307 sections of the information, as I will now explain.

\section{$2308 \quad 3.1 .5$ Sampling}

2309 Sampling may not be appropriate when working with certain types of document. The

2310 corpus of patient information developed for this doctoral research is not a general

2311 corpus: it is specialised, and the register of patient information has an organisational

2312 structure that is subject to guidelines and convention (e.g.MHRA, 2012). Patient

2313 information for radiography contains similar information and instructions, i.e.

2314 advice/directions regarding preparation, descriptions of the procedure, reference to

2315 risks and benefits, and to follow-up care, presented in a similar order (e.g. information

2316 regarding preparation appears at the beginning of a document, while follow-up care

2317 generally appears towards the end of the document). The aim of this study is to

2318 describe some of the linguistic devices used to express that information and those that

2319 instruct, so it was important to have complete documents in order not to remove

2320 sections of information and thus skew the results. Risk, for example, will generally not

2321 be discussed at the beginning of a document but towards the end. Offers of more

2322 information and advice come at the end of a document, not in the middle. The legal

2323 disclaimers that, as we shall see in the following chapter, are a very significant part of

2324 the US-sourced information, always appear at the end of the document. A description

2325 of the procedure and the benefits are far more likely to come at the beginning of a

2326 document.

2327 It is my view, then, that sampling would not have been appropriate for the 2328 documents in my corpus, irrespective of the difference in length between them. Nor is 2329 sampling something that needs to be done when texts are already brief in length. The 
2330 idea that sampling is not suitable for certain types of corpora is shared by many

2331 researchers (e.g. Flowerdew, 2004; Kennedy, 1998) and it is worth remembering that

2332 the individual documents in the patient information corpus are already short or very

2333 short in most cases. 'Frequent linguistic features are quite stable in their distributions

2334 and hence short text chunks (e.g. 2,000 running words) are usually sufficient. (Xiao,

2335 n.d.) If Xiao considers 2,000 words to be a short text chunk, the vast majority of the

2336 documents in my corpus can be considered short or very short.

\section{3.2 Reference or comparison corpora}

2338 When conducting a keyword analysis, a reference corpus, also known as a

2339 comparative corpus, is used to calculate the 'keyness' or statistical significance of

2340 words in the target corpus. The default reference corpus in Sketch Engine is English

2341 Web 2013 (EnTenTen 13), a corpus of internet texts running at around 19 billion

2342 words. For my analyses, however, I chose to use the British National Corpus (BNC), a

2343 100-million-word collection of British English, 90\% from written discourse. The BNC

2344 comes pre-loaded in Sketch Engine. Not only is the BNC a very common choice of

2345 reference corpus but the preponderance of written discourse in the corpus made it a

2346 suitable reference corpus for this study. It is also the case that my corpus was made up

2347 of Word and pdf documents, often the same documents that are available in hospital

2348 radiography departments and GP surgeries. The materials downloaded from

2349 RadiologyInfo.org were also produced to be both read online and downloaded. The

2350 size, too, was a factor. EnTenTen 13 is a web-based corpus and is enormous, at 19

2351 billion words. While 100 million words (the BNC) is still considerably more than my

2352 corpus of round 400,000 words, the comparison in size is more appropriate.

2353 As we have already seen in the literature review, however, comparing a

2354 specialised corpus against a general corpus like the BNC, is not the only approach

2355 likely to yield interesting results. In fact, comparing a specialised corpus against

2356 another specialised corpus may well reveal discourses that remain hidden with the

2357 former approach. Because of this advantage, I also built two reference corpora to be

2358 used for more targeted analysis, both in the keyword analysis and in the modal verb

2359 analysis. These two corpora I will now present. 
2361 This was a small, 104,670-word corpus of consumer information, with material from

2362 both the UK and the US. The inclusion criteria for the consumer information corpus

2363 was very close to that for the patient information corpus. All texts were available as

2364 Word or pdf documents on the Citizens Advice website, a recognised authority in the

2365 UK for consumer information, and its US equivalent, the Federal Trade Commission.

2366 This was a much smaller corpus than the patient information, however, with a word

2367 count of just 104,670 and the majority of texts came from the UK Citizens Advice

2368 site. The topics covered included housing, health, children, consumer topics and the

2369 law. The length of the documents included in this corpus also varied, from the longest

2370 at over 7,000 words to the shortest at under 300 words. The longest documents in this

2371 corpus were from the UK.

2372 The rationale behind building a reference corpus of consumer information lay in 2373 the fact that patients are increasingly referred to as consumers, or as clients or service 2374 users. But are patients treated as consumers in written informational materials? Are

2375 they spoken to in the same way? I wanted to investigate any lexical similarities or

2376 differences between medical patient information and general consumer information,

2377 particularly with regards to the use of deontic modal verbs, those verbs we use for

2378 instruction and obligation. It was my hypothesis that irrespective of the fact that

2379 patients are sometimes referred to as consumers, they are not treated as such in

2380 procedural patient information. The way that healthcare materials instruct patients is

2381 quite different to general consumer information, which is far more likely to direct and 2382 instruct readers. The topic will be explored in more detail in Chapter 6.

\section{3.2.2 Comparison corpus 2: General radiography}

2384 This was the first reference corpus that I built, and at 719,209 words it is considerably 2385 larger than the corpus of Consumer Advice. The corpus of General Radiography is 2386 made up of a radiographer handbook, Clark's Positioning in Radiography, a textbook, 2387 Patient Care for Radiography and research from Radiography, a peer-reviewed 2388 journal of the Society and College of Radiographers and the European Federation of 2389 Radiographer Societies. The research was included because it is written for and by 2390 radiographers, and not radiologists, and thus deals with the issues that are relevant for 
radiographers: patient safety, radiation dose, patient position and workflow, for

2392 example. Radiologists are doctors who specialise in radiology. Their job is to

2393 diagnose, primarily. Radiographers, on the other hand, are the healthcare professionals

2394 who carry out radiographic examinations.

2395 The reason for using this corpus of radiography when extracting keywords has 2396 already been touched upon in Chapter 2. A specialised reference corpus is often the 2397 best choice when working with a specialised corpus, otherwise the keywords are 2398 likely to be solely the technical terms in the corpus, which may not be of much 2399 interest to the researcher. Patient information for radiography and radiotherapy 2400 contains many references to radiographic modalities (types of exam), (e.g. MRI, CT), 2401 radiographic procedures (e.g brachytherapy), and to medical or technical terms, even 2402 if they are then glossed for the patient information. I was not interested in these words 2403 for the purposes of my research, but more interested in what might be considered 2404 general language. Using the BNC as a reference corpus, however, naturally results in 2405 many of medical and radiological terms appearing as keywords. By conducting a 2406 second keyword extraction using a radiography reference corpus, and thus reducing 2407 the chance of medical and technical words being extracted as key, I felt that the 2408 analysis would be more fine-tuned and perhaps reveal more about the underlying 2409 characteristics and concerns of patient information.

2410 I now turn to a description of the software programme used to build and analyse 2411 my corpora, describing its functionalities and tools before moving on to present the 2412 specific steps take for each of the three methodologies.

\section{3.3 Software: Sketch Engine}

2414 Sketch Engine (Kilgarriff et al., 2004) is a corpus manager and text analysis software 2415 programme, developed by a company called Lexical Computing. It is designed 2416 primarily for lexicographers, translators and researchers, and along with the corpus 2417 building function it offers, it also contains a large number of pre-loaded multilingual 2418 corpora and a range of lexical analysis functions for use with either self-built or the 2419 pre-loaded corpora. These functions include the proprietary Word Sketch, which is a 2420 summary of a word's grammatical and collocational behaviour; along with a 
2421 concordance search; collocation search; word frequency lists; keyword and

2422 terminology extraction; diachronic analysis and n-gram extraction. $\mathrm{N}$-gram is

2423 synonymous with lexical bundle, where $\mathrm{n}$ stands for any number.

2424 For this research thesis, I used the concordance search to examine the lexis in 2425 context; I used, too, the keyword function to generate lists of keywords and also the n2426 gram function, which is a synonymous term for lexical bundle. I used Word Sketch to 2427 look at an item's common lexical and grammatical collocations, particularly when I 2428 was investigating the deontic modal verbs and keywords. The first step, however, was 2429 to build the corpora. The steps will be illustrated below and were the same for all three 2430 corpora.

\section{$2431 \quad 3.3 .1$ Building the corpus}

2432 Sketch Engine (www.sketchengine.eu) Kilgarriff et al., 2004) was used to compile the 2433 corpus and to perform the analyses described in the following chapters. The 2434 illustrations below relate to the original interface which I had used during my 2435 research. A new interface was introduced in 2018.

2436 One of the advantages of Sketch Engine is that it does not require text to be 2437 converted to raw text files, which is standard to most or all other similar concordance 2438 programmes. In fact, Sketch Engine accepts a range of document types, including 2439 Word and pdf files, meaning no time-consuming conversion was needed. To my 2440 knowledge, the programme is unique among concordance programmes in its ability to 2441 process a wide range of file types. Sketch Engine supports corpora in many languages 2442 and also offers many pre-loaded corpora, in multiple languages, which can be used for 2443 analysis or for comparative purposes. I did not use any of the pre-loaded corpora as 2444 none of them was suitable for my purposes, however. While there is a dedicated 2445 medical English corpus, it is made up of data found on the World Wide Web and is 2446 enormous in size, at 33 million words, rendering it unsuitable for my aims on the basis 2447 of size and its contents, As I have stated, this study is not concerned with peer-to-peer 2448 communication, web-based chat programmes or online interaction, but, rather, 2449 available-for-printing healthcare materials, and thus the content of this medical 2450 English corpus on Sketch Engine - not to speak of its size - was unsuitable. 
2452 After selecting Create corpus on the menu, documents were uploaded as zip files for

2453 multiple texts, or individually. I had previously downloaded documents as Word or

2454 pdf files and placed many of them into zip files. It is also possible to download

2455 directly from an online location or to paste text, as you can see in Figure 1.

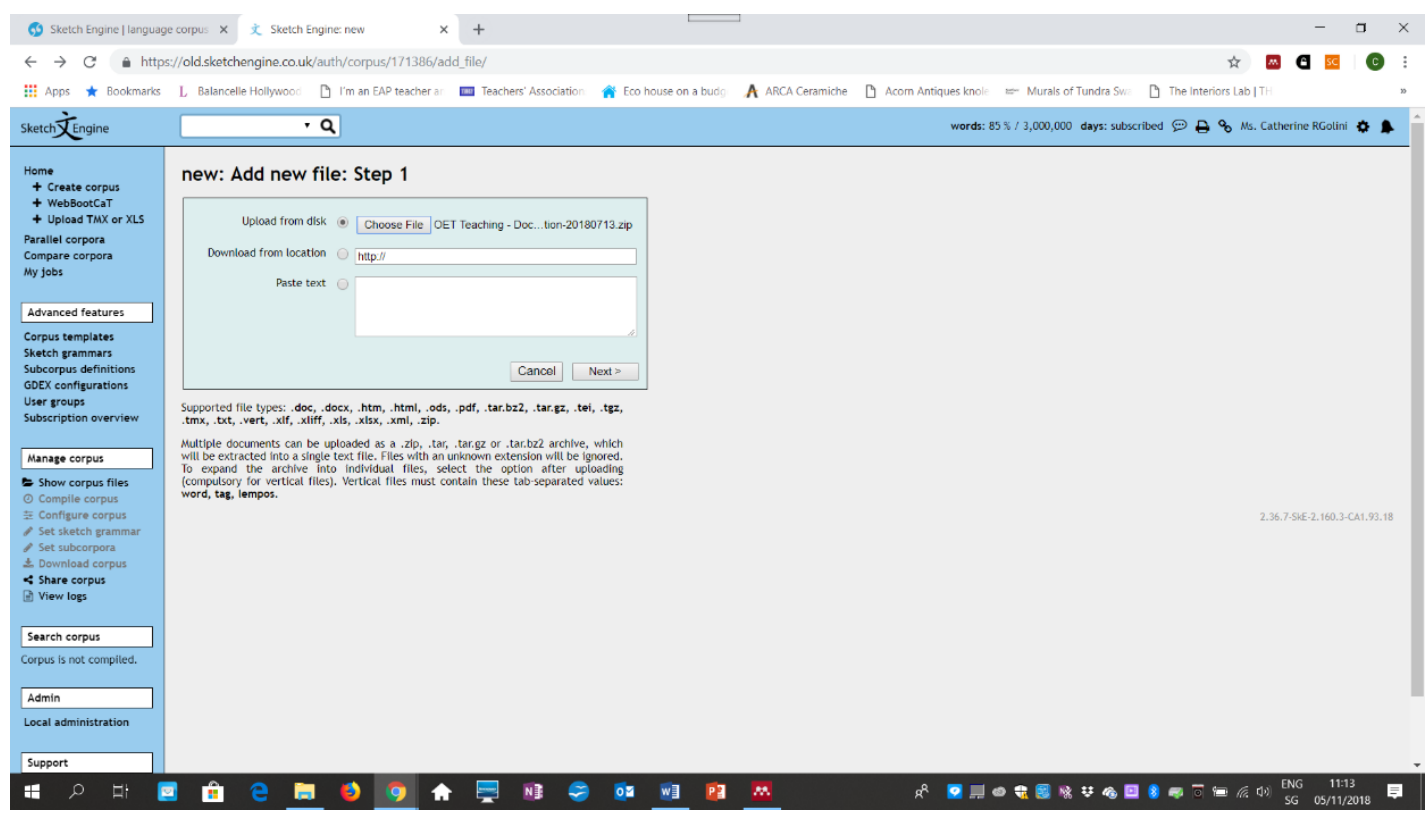

2458 Figure 1 Adding text to the corpus

2459 3.3.3 Compiling the corpus

2460 The next step, illustrated in Figure 2 below, was to compile the data, processing it so

2461 that the various functions of the programme described above can be used. I accepted

2462 all default settings in Sketch Engine: for the Sketch grammar English 3.3 for

2463 TreeTagger pipeline v2 and for the term extraction, English (TreeTagger-PennTB) for

2464 terms extraction 2.3. TreeTagger refers to the part-of-speech tagging that the text files

2465 are subject to. Part-of-speech tags relate to the grammatical category of a token (i.e.

2466 verb, singular noun) and also, in some instances, to case and tense. 


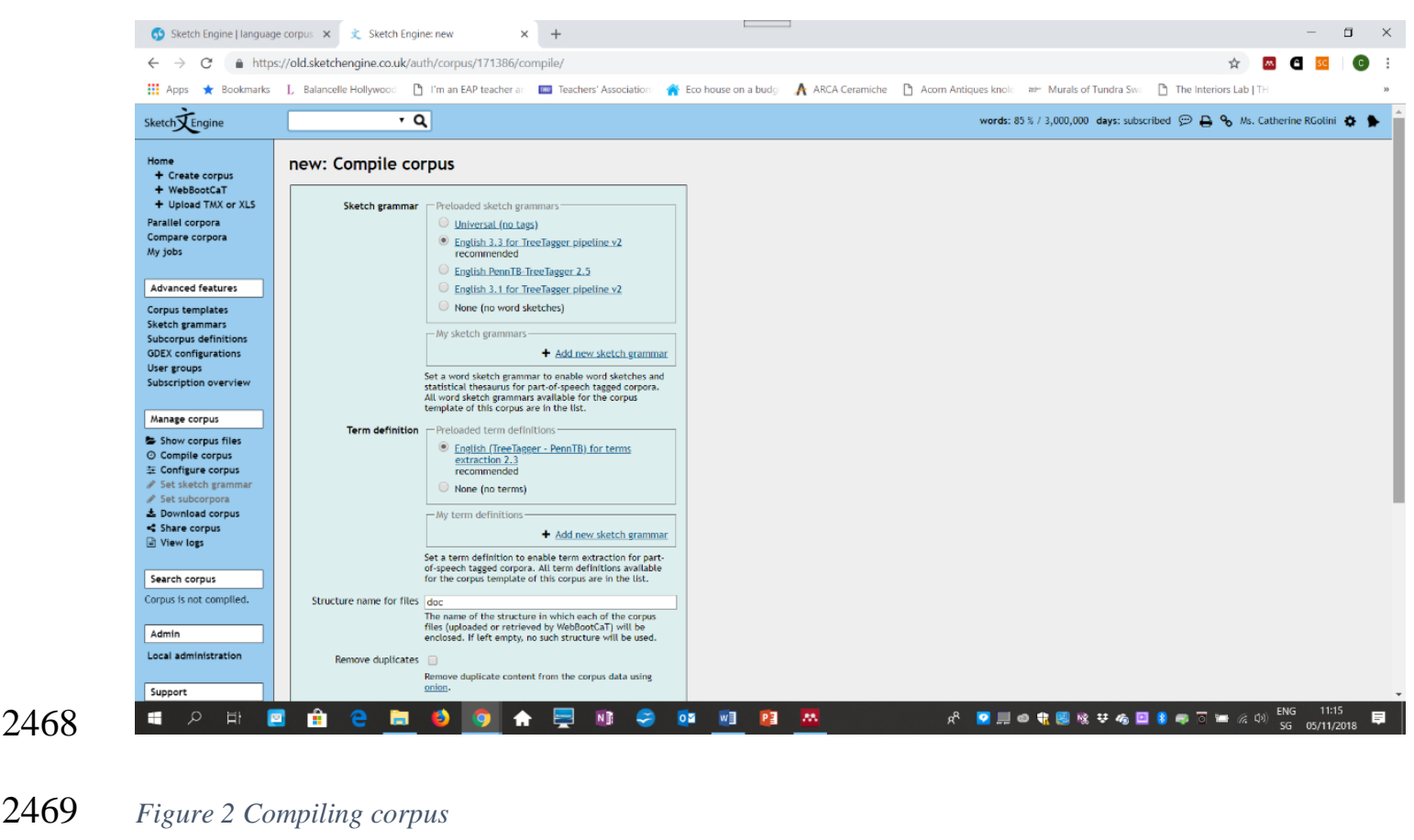

2470 At this point, the user can select to make use of the 'Onion' programme, which

2471 removes all duplicate content at whatever level the user sets (i.e. the sentence,

2472 paragraph or document level). There is a lot of similarity in patient information for

2473 radiography; templates are used and the same phrases are repeated, removing

2474 duplicate content would have reduced my corpus considerably. However, the standard

2475 (i.e. repeated) phrases used in a register are part of that register's characteristics. One

2476 of the aims of this doctoral research was to reveal some of the lexical characteristics

2477 of patient information for radiography, included the oft-used sentences. Clearly, for

2478 my purposes, removing duplicate content was not an option that I wanted, so it

2479 remained unchecked.

2480 Compiling a corpus is something that must be done whenever new content is

2481 added to the corpus. If the uploaded text is not compiled, it cannot be searched.

2482 Documents can be added to the corpus at any time after the initial compilation. Once

2483 the corpus has been compiled, it can be searched and the functionalities referred to

2484 previously, e.g. term extraction, n-gram extraction, can be applied.

2485 3.3.4 Searching the corpus

2486 Sketch Engine as I have said in 3.3, offers the user a range of options for corpus

2487 searching and analysis. Word Sketch, which gives the user the grammatical and 
2488 lexical collocation information of a selected word, is proprietary. It is also possible to 2489 compare collocational information for two different words. As an example of the kind 2490 of lexical information presented in Word Sketch, a screenshot for the word pain can 2491 be seen in Figure 3 below.

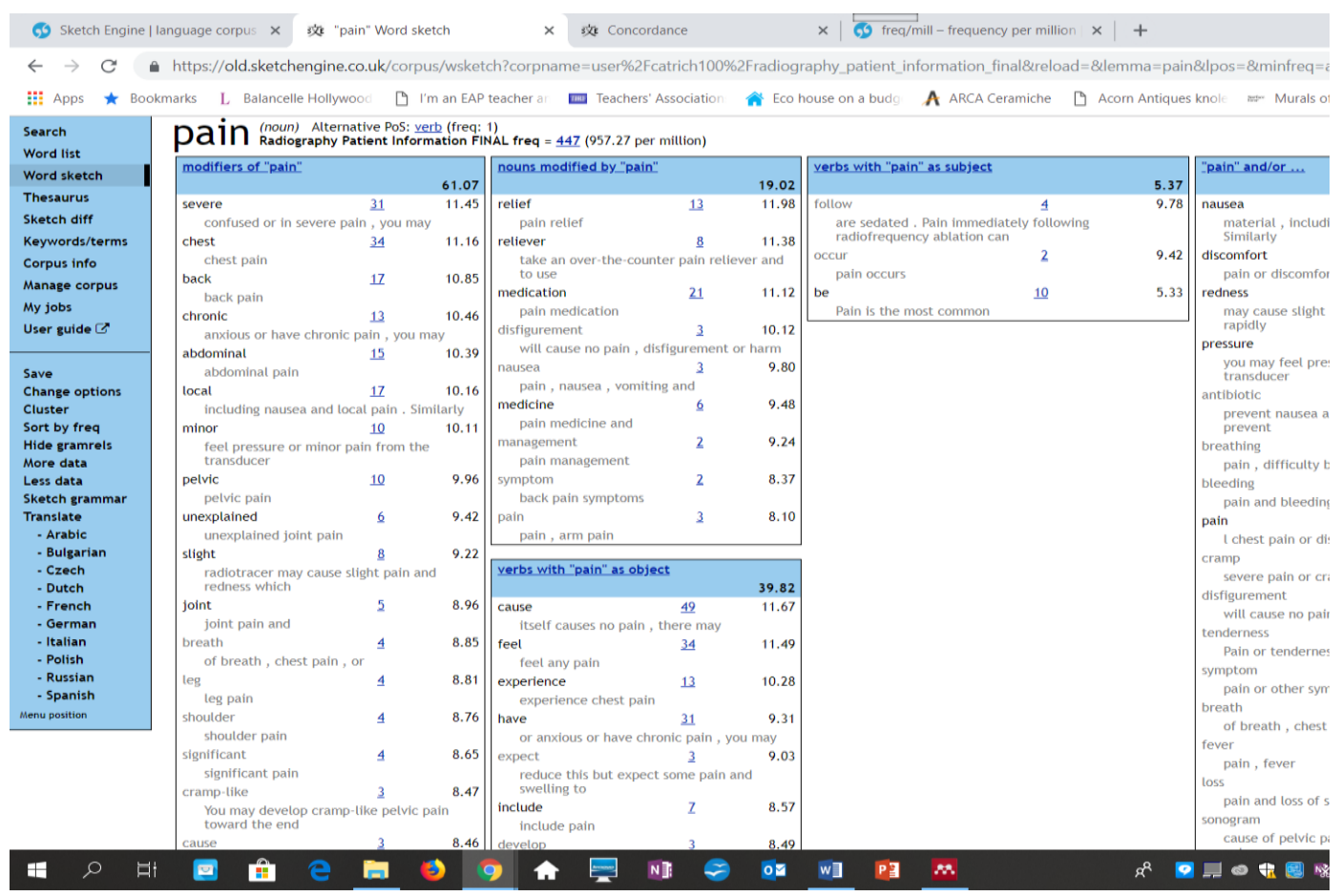

2493 Figure 3 Word Sketch showing results for 'pain'

2494 To the right of the word pain, at the top of the page, we can see the most common part 2495 of speech for the selected word (in the case of pain it is a noun) and any other part of 2496 speech, if it was found in the corpus. There is just one example for pain, of a verb. On 2497 examining this data in context (by clicking on the number 1), we see that it is not, in 2498 fact, a verb, but a noun, underlining the importance of context and the fallibility of 2499 computer programmes. You may feel pressure or even pain when the needle is advanced into the joint. The collocation and grammatical information are arranged according to 2502 frequency, in descending order. The count appears both as raw count (a simple 2503 frequency count of how many times the search term appeared in the corpus) and as a 2504 normalised count, which will be explained in the next section. The raw count is 
2505 hyperlinked and when clicked on, takes you to the data in context. In the following

2506 section I will present raw and normalised frequency counts.

$2507 \quad 3.4$ Issues of frequency reporting and distribution

2508 3.4.1 Raw frequency and normalised frequency

2509 When conducting my modal verb analysis, frequency counts were normalised, that is,

2510 expressed as per million words ( $\mathrm{pmw}$ ). Normalising frequencies allows comparisons

2511 to be made between differently sized corpora, as raw frequencies do not accurately

2512 reflect relative frequencies. A lexical item that appears 50 times in a corpus of

2513200,000 words is not less frequent than one that appears 500 times in a corpus of 2

2514 million words. Per million words seems increasingly to be the standard, as corpus

2515 sizes increase, although some researchers have used 100,000 (Biber, 1998, p. 32)

2516 some 10,000, and sometimes even 1,000 is used. (e.g. Goźdź-Roszkowski, 2011). In

2517 this thesis, notwithstanding the fact that the corpus was a little over 400,000 words, I

2518 used pmw as it was a default setting in Sketch Engine, which could not be changed

2519 (though of course, the normalised frequency can also be calculated by hand).

2520 The analysis where the normalised frequency is more relevant was the

2521 investigation of the modal verbs, reported in Chapter 6. Lexical bundles and keyword

2522 occurrences are not reported in terms of raw or adjusted frequencies. As I was more

2523 interested in the use of modal verbs in patient information, and this was not a study to

2524 compare registers - i.e. it was the frequency of use of a modal verb relative to other

2525 modal verbs in the patient information that interested me, rather than the frequency of

2526 use relative to another register - I felt that the setting for the normalised frequency was

2527 not a concern. As for the decision to report both raw and normalised frequency, I have

2528 followed the advice of McEnery and Hardie who say: 'It is usually considered good

2529 practice to report both raw and normalised frequencies when writing up quantitative

2530 results from a corpus' (McEnery \& Hardie, 2012, p. 51).

2531 The minimum frequency default setting in Sketch Engine is 5, which means 5

2532 raw frequency counts in the corpus. 8 raw counts, when normalised in my corpus of

2533 patient information of around 400,000 words, represents about 20 pmw. 20 pmw was 
2534 the cut-off point used in the lexical bundle analysis, the methodology of which will be 2535 discussed in more detail later in this chapter.

\section{3.4.2 Distribution}

2537 Distribution is also an important factor when conducting a corpus analysis. A word 2538 may appear 50 times in a corpus, but if 49 of those uses are found in one document, 2539 and thus the work of one writer, its use is considered idiosyncratic and not

2540 generalisable. Establishing a minimum range is standard practice in corpus studies and 2541 five is both common in the literature and was the Sketch Engine default. In my 2542 research, too, five was set as the minimum distribution.

2543 Having presented the corpora I built and the software I used to carry out my 2544 analyses, I now turn to the methodologies of these analyses: keyword extraction, 2545 lexical bundle analysis and an analysis of the modal verbs used for instruction and 2546 obligation.

\section{$2547 \quad 3.5$ Methodologies: Corpus-driven and corpus-based approaches}

2548 There are generally two approaches to corpus analysis, what Tognini-Bonelli (2001) 2549 refers to as corpus-based, where lexical items are pre-selected and then searched for 2550 within a corpus, and corpus-driven studies, where there are no preconceived lists of 2551 expressions and 'recurrent patterns and frequency distributions are expected to form 2552 the basic evidence for linguistic categories; [and where] the absence of a pattern is 2553 considered potentially meaningful' (Tognini-Bonelli, 2001, p84). The three lexical 2554 analyses that make up this study of patient information and the approach taken varies 2555 accordingly. I used a corpus-driven approach in my analyses of keywords and lexical 2556 bundles reported in chapters 4 and 5, while in chapter 6 and my analysis of a range of 2557 modal verbs, the approach was corpus-based. The methodologies used are quite 2558 different from each other and will be presented below. I begin by describing the 2559 methodology of a keyword extraction. 
2562 The simplest definition of a keyword is that it is a statistically significant lexical item

2563 (Scott,1997). Keywords are generated by a computer using statistical calculations and

2564 thus this stage of the analysis is quantitative. The extraction of keywords is then

2565 followed by a semantic categorisation in order to establish the underlying themes in

2566 the discourse. These categories - all or some depending on the researchers' agenda -

2567 are then examined in context, taking careful note of how the words are used,

2568 especially their collocational partnerships. A keyword analysis is, therefore, both a

2569 quantitative and a qualitative analysis.

2570 A keyword analysis is a very useful tool in healthcare language studies,

2571 especially those where real-world outcomes are a key objective. We have already seen

2572 a number of these studies in Chapter 2, where these real-world outcomes relate to the

2573 end-users, who are generally professionals or patients. For example, a deeper

2574 understanding of a psychologically-motivated condition (e.g Harvey and Brown,

2575 2012) or evidence of the severity of adolescents' anxiety concerning the revealing of

2576 confidences (e.g Harvey et al., 2008) can be used to inform healthcare professionals'

2577 training and to improve adolescents' experiences of healthcare. Many register studies

2578 have shown keywords to successfully reflect the characteristics of a register, and the

2579 approach seems to have greater sensitivity than some purely qualitative methods (e.g.

2580 open-ended interviews) (e.g. Seale et al., 2006). The approach can give important

2581 lexical information about the information priorities of the register under investigation

2582 and can also reveal the discourse and themes prevalent in a text which may be hidden

2583 when examining the text with a purely qualitative approach. I believe that a keyword

2584 analysis is also complementary to an analysis of lexical bundles, as while the former

2585 can give us more information about the themes and beliefs in a text, the latter can tell

2586 us more about the underlying communicative function of a text. Lexical bundles will

2587 be the subject of the next chapter.

2588 This section begins with a definition of a keyword, an explanation of the

2589 reference corpus, and mention of the settings and statistical tests that are applied in 
2590 Sketch Engine when generating keywords. This will be followed by a section

2591 describing the second stage of the methodology: the semantic categorisation.

\subsubsection{Defining a keyword}

2593 The simplest definition of a keyword is, as we have seen in 3.5.1.1, that it is a

2594 statistically significant lexical item (Scott,1997). The item is statistically significant

2595 because its frequency in a corpus is compared to that in a second, reference corpus.

2596 All keyword analyses, then, involve the use of a reference corpus which serves as a

2597 comparison corpus; a statistical analysis is carried out which produces a frequency list

2598 of lexical items in the corpus under investigation when compared to the reference

2599 corpus - usually, though not always as we shall see, a large-scale, general corpus such

2600 as the British National Corpus (BNC).

2601 An item may appear as a keyword with both positive or negative frequency, that

2602 is, appear more or less frequently than might be expected by chance. In Sketch

2603 Engine, however, keywords have only a positive frequency: a keyword is listed when

2604 it appears more frequently in the corpus under investigation than might be expected by

2605 chance. Keywords are not synonymous with terminology, though Sketch Engine

2606 offers the user the possibility of extracting what they refer to as 'terms' in addition to

2607 carrying out a keyword analysis. Terms, as defined by Sketch Engine, are two-word

2608 noun phrases (collocations to a language teacher or researcher) that appear with a

2609 greater frequency when compared to a reference corpus. A keyword as it is used in

2610 this thesis and the studies reported in Chapter 2 is not solely a noun but can be any

2611 kind of word class, including pronouns or conjunctions.

2612 In Sketch Engine, as I will now explain, the user has some control over the

2613 balance between content and grammatical words in their keyword list. When setting

2614 up the software to extract the keywords, Sketch Engine offers the option of varying

2615 the degree of 'rareness' of the keyword, what they refer to as the 'smoothing

2616 parameter' as Figure 4 below shows. Varying the smoothing parameter results in

2617 more, or less, content words being extracted. In contrast, the closer to 'common' the

2618 setting, the greater the likelihood of grammatical words being extracted. 


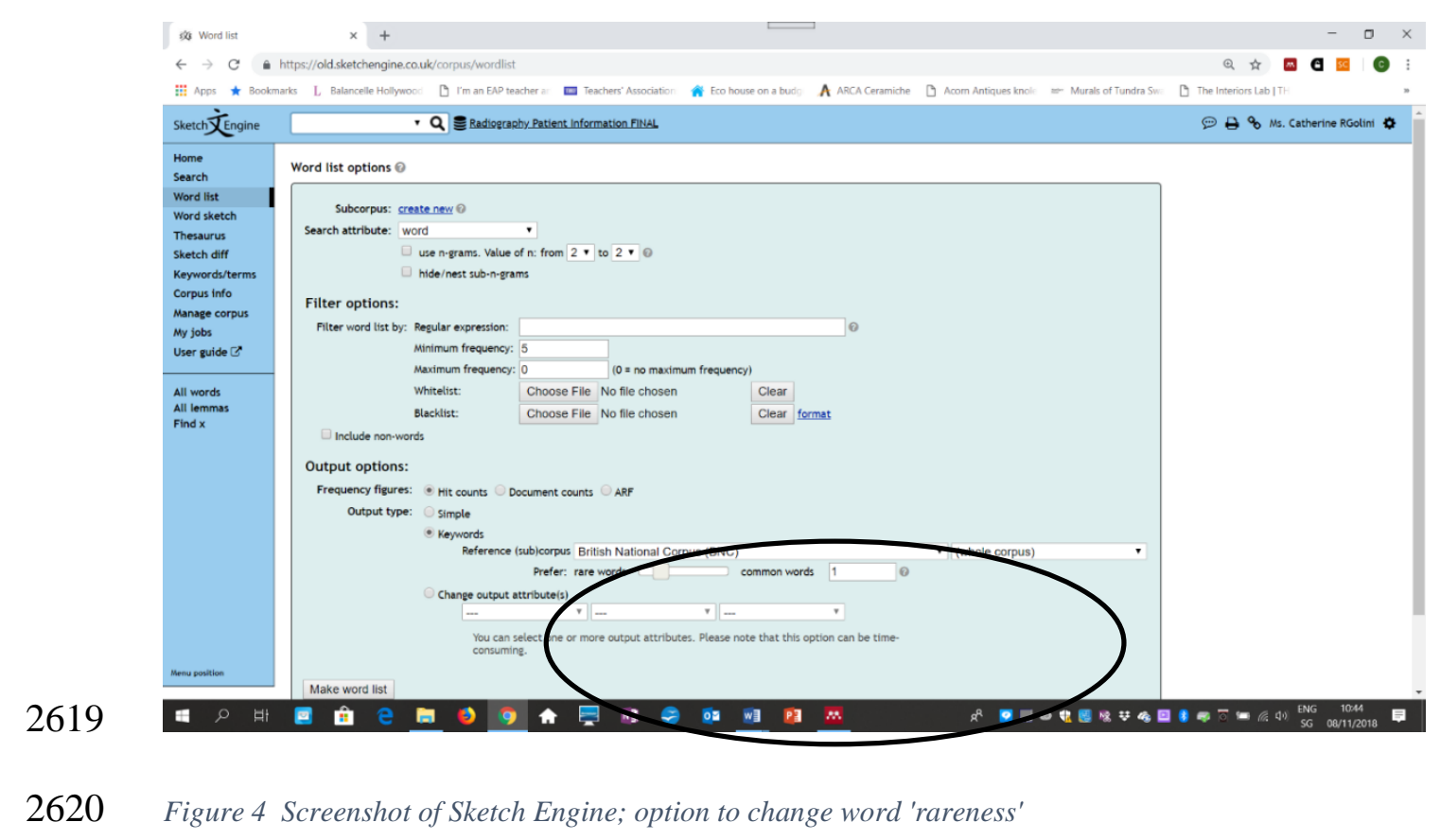

2621 For example, the setting at 1,000,000 (with the British National Corpus as the

2622 reference corpus) gives us the most common items, and we get the following in the

2623 first ten keywords: the or, is, may, your, will, you, be, procedure, are. As we see, there

2624 is only one content word when the setting is at 1,000,0000: procedure. CT appears in

2625 eleventh place (though placing or where a keyword is in the list is not necessarily

2626 important when dealing with keywords, as I explain in 3.5.1.8). In contrast, when the

2627 setting is at 1, the Sketch Engine default setting, we get the following words: $C T$,

2628 MRI, imaging, x-ray, copyright, radiologist, RadiologyInfo.org, reviewed, physician,

2629 ultrasound, which are all content words. When the smoothing parameter is set to

26301000 , on the other hand, we get the following: procedure, may, your, CT, images,

2631 MRI, imaging, information, radiation, or; a mix between content words and

2632 grammatical, with the former predominant.

2633 What does this mean for the researcher? All of these lexical items are

2634 potentially interesting, of course, though there will be research questions that render

2635 content words more or less interesting and thus govern the choice of setting. I elected

2636 to set the smoothing parameter at 1 for this study, i.e. the default setting. I did not

2637 want to exclude grammatical words, but the content words, in particular, were my

2638 focus. There was no precedent for this, but given that this is the first study of

2639 keywords in patient information, it seemed appropriate. Later studies may wish to 
2640 focus on grammatical as well as lexical items in the patient information, and thus

2641 choose a different parameter setting.

\section{3.5.1.3 Statistical calculations}

2643 There have been a number of statistical analyses that have been carried out to generate 2644 keywords, though the most frequently used for keyword extraction within applied 2645 linguistics are Mutual Information (MI) (Church and Hanks, 1990), Log-likelihood 2646 (Dunning, 1993) and the t-test. In Sketch Engine, the statistical calculation used is 2647 what is referred to as 'Simple Maths', where the calculation is as follows:

$2648 \frac{f p m_{\text {rmfocus }}+N}{f p m_{\text {rmref }}+N}$

2649 where

$2650 \mathrm{fpm}_{\text {rmfocus }}$ is the normalized (per million) frequency of the word in the focus corpus,

$2651 \mathrm{fpm}_{\text {rmref }}$ is the normalized (per million) frequency of the word in the reference 2652 corpus,

$2653 N$ is the so-called smoothing parameter $(\mathrm{N}=1 \text { is the default value })^{2}$

2654 3.5.1.4 Reference corpus

2655 As I have stated already in this chapter, keywords are words that are statistically more 2656 frequent (or less frequent) in the corpus under-investigation than they are in a 2657 reference corpus, also referred to as a comparison corpus. The standard approach 2658 when investigating general vocabulary is to use a general reference corpus, such as the 2659 BNC. However, an increasing number of studies of specialised discourse are electing 2660 to use a specialised corpus as a reference corpus.

2661 As we saw in chapter 2, Seale et al. (2006) and Seale and Charteris-Black (2008) 2662 did not use a general reference corpus in their study. In the 2006 study, the authors 2663 were interested in learning how forum conversations by women about breast cancer

\footnotetext{
${ }^{2}$ https://www.sketchengine.eu/documentation/simple-maths
} 
2664 differed from those by men about prostate cancer, each set of forum conversations was

2665 used as the reference corpus for the corpus under investigation, i.e. the corpus of

2666 breast cancer conversations had, as a reference corpus, the conversations about

2667 prostate cancer, and vice versa. I have suggested that such an approach is not usual,

2668 though there are other keyword studies, some of which have been referenced in the

2669 literature review in chapter 2, that use either a dual reference corpus or a single,

2670 domain-specific reference corpus (e.g. Baker, 2004; Goźdź-Roszkowski, 2011 and

2671 Grabowski, 2015). Explaining why this might be an appropriate step to take, Goźdź-

2672 Roszkowski (2011) says 'Comparing a range of specialized genres ${ }^{3}$ from the same

2673 domain against a general reference corpus would inevitably lead to obtaining finding

2674 which may be highly homogeneous and probably valid for legal language in general

2675 but would not help identify features unique to a particular genre' (p36).

2676 In my study of keywords reported in chapter 4, I performed two separate

2677 keyword extractions and thus used two different reference corpora: the BNC and a

2678 domain specific corpus of general radiography. The BNC was used as it is widely

2679 available, comes pre-loaded in Sketch Engine and is often used as a reference corpus

2680 in the literature, meaning that it provides 'a recognizable common ground for keyword

2681 [...] comparisons' (Charles, 2009, p20). The domain-specific corpus of general

2682 radiography contains 719,209 words and is made up of a radiographer's handbook,

2683 Clark's Positioning in Radiography, a textbook, Patient Care for Radiography and

2684 research from Radiography, a specialised academic journal about radiography and

2685 thus with research by and for radiographers. The full details of this domain-specific

2686 corpus have already been reported in 3.2.2, while the reasons for using a domain-

2687 specific reference corpus in addition to the more-usual general corpus I have outlined

2688 earlier: a more targeted description of the register may be achieved which, alongside

2689 the results of the BNC comparison, can help build a more informed picture of the

2690 lexical characteristics of radiography patient information.

\footnotetext{
${ }^{3}$ Genre, as used by Goźdź-Roszkowski, is synonymous with my use of register in this thesis.
} 
Having presented the software settings relevant to a keyword extraction, I now turn to the steps taken to extract a keyword list.

\subsubsection{Extracting a keyword list}

2694 The first step in a keyword analysis is to extract a keyword list. In Sketch Engine, this

2695 can be done in one of two different ways: the first is found under the tab

2696 Keywords/terms while the other can be found under Word List. I elected to use the 2697 latter, though it is my understanding that both offer the same options, i.e. reference 2698 corpora, minimum frequency, search terms, etc. and both give the same results.

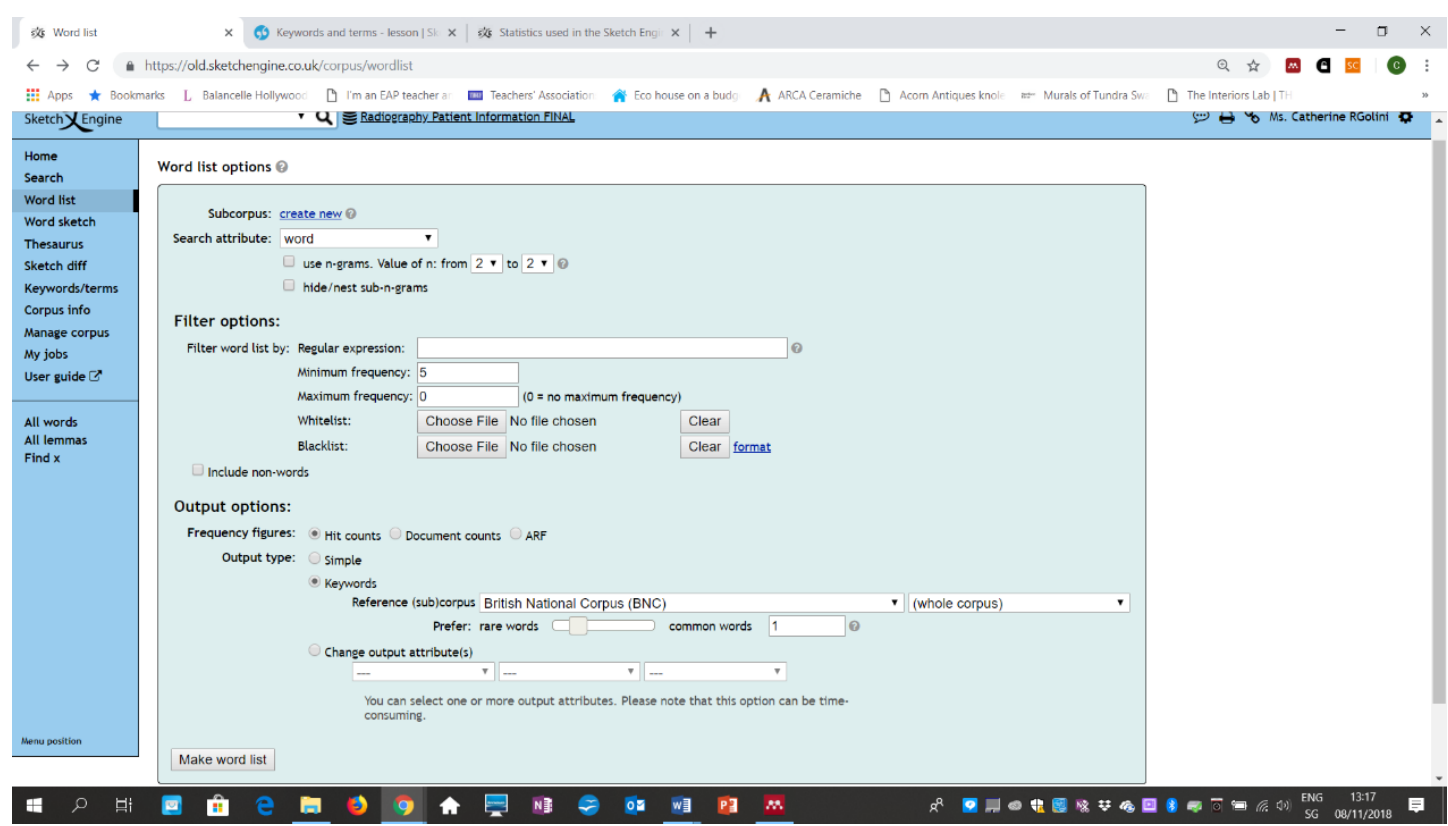

2702 The first setting to select is the Search attribute. I selected 'word' to be able to see 2703 unique examples of any form of a word in the final list. If the lemma option is chosen,

2704 the keyword scan, for example, would also include instances of scans, scanned and 2705 scanning, though clearly for a keyword list, these words need to be treated separately.

2706 I did not use any of the settings in the Filter options as they are not, with the 2707 exception of the Blacklist, relevant for a keyword search. I did not change the 2708 minimum frequency as keywords are ordered by keyness and I intended to investigate 2709 the first 50, irrespective of how many appeared on my final list. A Blacklist is used to 
2710 exclude words. For my keyword extraction, I did not wish to exclude anything. A

2711 Whitelist searches only for the words on the Whitelist - this is clearly not a keyword

2712 list if items are being included a priori.

2713 The Output options are where we set the Keyword search (as opposed to a

2714 Simple search, which does not require a reference corpus and produces a frequency

2715 list instead), and where we select the reference corpus and adjust the smoothing

2716 parameter if required.

\section{3.5.1.7 Keyword list}

2718 Once we have selected our parameters, the button 'Make Word List' will produce a

2719 keyword list. The results for a keyword extraction using a section of the BNC: Written

2720 Domain Informative can be seen below in Figure 6. (This was not the study carried

2721 out for this doctoral research but serves purely as an example).

2722 There are 5 columns. The first two show the raw frequency and the per million

2723 words (pmw) normalised frequency of the keywords. As I have said earlier in this

2724 chapter, the pmw normalised frequency is the default setting in Sketch Engine and is

2725 not a parameter that can be changed. To the right are two further columns, showing

2726 the same figures for the reference corpus. The fifth and final column is the Score. This

2727 refers to the keyness score, which is a calculation of the frequency of a lexical item in

2728 the source corpus, compared to the reference corpus. 


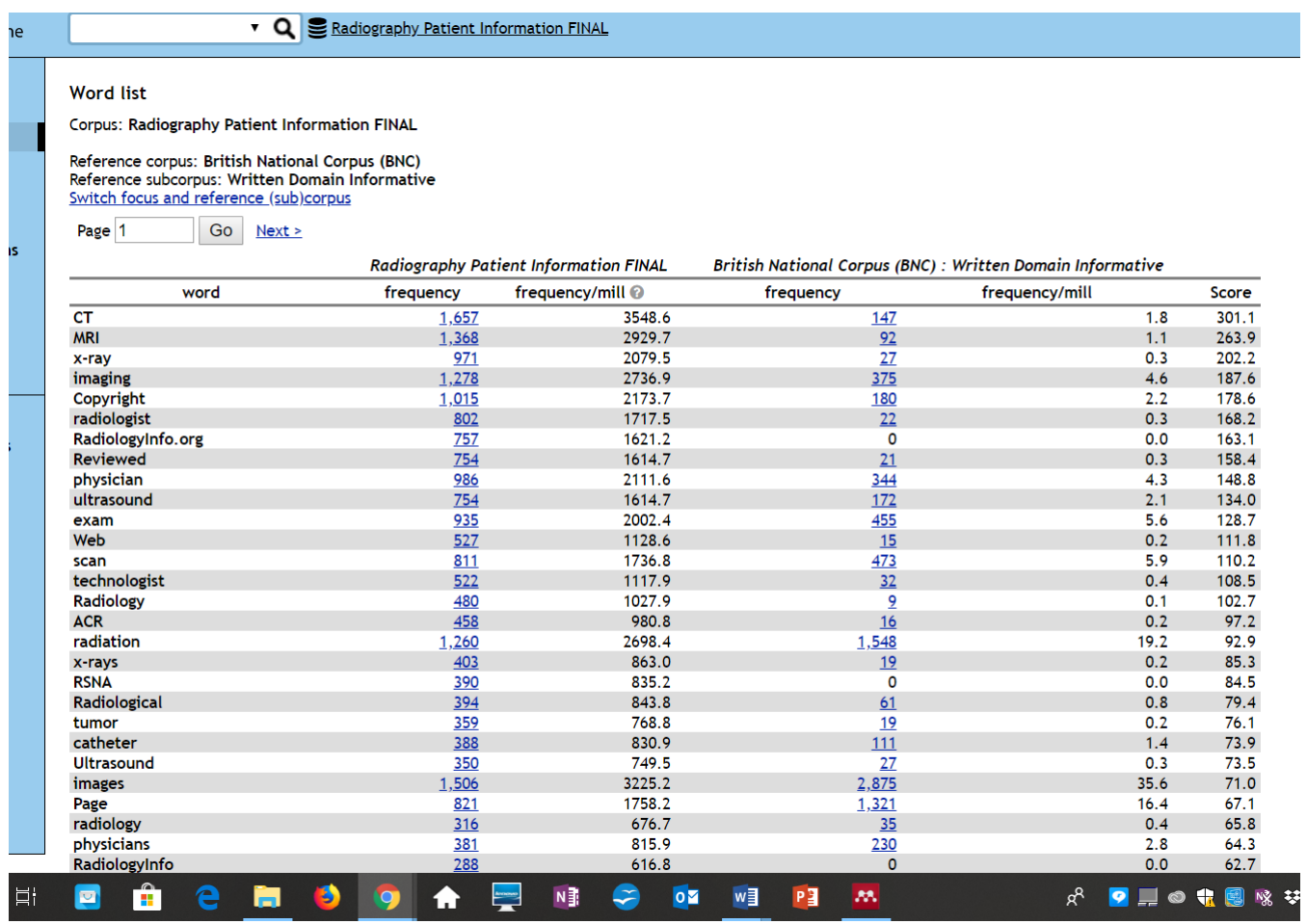

$2730 \quad$ Figure 5 Keyword list

\section{3.5.1.8 Keyword order}

2732 Keywords have been ordered by keyness score, as we see in Figure 6. This does not

2733 mean, however, that the order of keywords extracted by the analysis is necessarily

2734 significant, as it would be in a frequency list. Scott (2010) says 'the order of KWs

2735 [keywords] is not intrinsically trustworthy, because it depends not only on the

2736 frequency in the text we are studying...but also on their frequencies in the reference

2737 corpus' ( $\mathrm{p} 50$ ). The greater the number of keywords extracted, the greater the

2738 possibility that the inclusion of some is based on statistical chance, says Scott (2010,

$2739 \mathrm{p} 50)$. Given this, it is wise to see keywords as suggesting 'to the prospector areas

2740 which are worth mining but they are not themselves nuggets of gold.' (Scott, 2010,

2741 p51). It is also why the keyword extraction is followed by a semantic categorisation

2742 of all or some of the keywords and their subsequent investigation in the context of the 2743 corpus.

2744 The semantic categorisation, which is an important step in the methodology,

2745 will now be presented. 
2747 Keywords are then grouped semantically, either using the researcher's intuition, with

2748 or without the aid of a dictionary or by using software. I categorised the first 50

2749 keywords for both of the analyses manually, by inspecting their use in the corpus and

2750 by using the Word Sketch facility in Sketch Engine. I decided to inspect the first 50

2751 keywords, rather than the first-100 or 150, as I was carrying out two separate keyword

2752 extractions: one with the BNC as reference corpus and the second with the general

2753 radiography corpus. These have been described in 3.2. Word Sketch, a proprietary

2754 tool, gives lexical and grammatical collocational information about a word. It was

2755 referred to earlier in this chapter in 3.3 and illustrated with a screenshot of the Word

2756 Sketch for the noun pain.

2757 Inspecting a keyword in context is vital; sometimes a word can appear in two

2758 categories or the most common meaning of a keyword turns out not to be the way it is

2759 being used in the corpus. An example from my data is up-to-date. I initially thought it

2760 referenced the information, but closer inspection revealed that, while it does modify

2761 the noun information, it is used only in formulaic, legal disclaimers that are included

2762 in some patient information, and thus it was removed from the category Information

2763 (which was generic in nature and included leaflet) and added to the Legal category.

2764 There were no words in my keyword lists that could be placed in two categories, that

2765 is, there were no keywords used with two distinct meanings.

2766 Once the semantic categories have been established, the words can be

2767 investigated in the corpus, and their connotations and their collocations examined. As

2768 I have already said, I used Word Sketch to do this and examined the sections in the

2769 corpus where these keywords were used. At this point, I was able to draw some

2770 conclusions about the use of the keywords in the corpus and what they reveal about

2771 the salient themes in patient information for radiography. These themes will be

2772 presented and discussed in Chapter 4.

2773 This concludes the presentation of the keyword methodology. In the next section,

2774 I will present the methodology of the lexical bundle analysis, the second corpus-

2775 driven method used in this analysis of patient information for radiography. 
2778 Lexical bundles are multi-word lexical sequences that frequently reoccur in a register,

2779 e.g. in the light of and at the end of. They have been described as "characteristic

2780 features of language use in particular settings' (Hyland, 2008, p8) and as 'text

2781 building blocks' (Biber et al., 2004, p443). Usually transparent in meaning, they tend

2782 to be incomplete and often bridge two structural units, i.e. a clause or phrase, very

2783 often functioning as the pragmatic head of an utterance and acting as an interpretative

2784 frame for the discourse that follows (Biber and Barbieri, 2007, p8.)

Variously referred to in the literature as formulaic sequences (Wray 2002;

2786 Schmitt and Carter 2004), lexical bundles (Biber \& Conrad, 1999), n-grams (Stubbs

2787 and Barth 2003) or lexical phrases (Nattinger \& DeCarrico, 1988), lexical bundles

2788 have received increasing attention over the last two decades, though, as we have seen

2789 in Chapter 2, there have been very few investigations of lexical bundles in medical

2790 registers. For McEnery and Hardie (2012, p110), lexical bundles are,

2791 'methodologically and technically', simply recurring sequences of $n$ words, i.e. n-

2792 grams, though they add that the term lexical bundle has become associated the work

2793 of Biber and colleagues on register description, and on their focus on the structural

2794 and functional interpretation of lexical bundles. As it is the structural and functional

2795 interpretation that interests me, and thus it is Biber and colleagues' terminology and

2796 approach that I have chosen to use, lexical bundle is the terms I used.

2797 While earlier studies of chunks of language relied on intuitive lists of 2798 prefabricated expressions (e.g Pawley and Syder, 1983; Nattinger and DeCarrico, 2799 1988; 1992), corpus software has permitted an evidence-based approach to studies of 2800 bundles, with Altenberg's study (1998) of the phraseology of spoken English, being 2801 one of the earliest.

2802 We have seen already that corpus studies fall broadly into two camps: corpus2803 based, where lexical items are pre-selected and then searched for within a corpus, and 2804 corpus-driven studies, where there are no preconceived lists of expressions and 2805 'recurrent patterns and frequency distributions are expected to form the basic evidence 
2806 for linguistic categories; [and where] the absence of a pattern is considered potentially

2807 meaningful' (Tognini-Bonelli, 2001, p84). Such studies also establish cut-off points

2808 and dispersion requirements in order to identify the lexical bundles that are frequent

2809 and worth investigating in the corpus. A lexical bundle analysis is a corpus-driven

2810 study.

2811 In the section that follows, I present the methodological steps taken to conduct a

2812 lexical bundle analysis, which, for this doctoral study, was an analysis of 4-word

2813 lexical bundles. These bundles are less common than 3-word bundles, which occur

2814 very frequently in both spoken and written discourse: Conrad and Biber (2004) claim

2815 that $25 \%$ of the words in conversation are found in 3-word bundles, while the most

2816 frequent 3-word bundle in conversation (I don't know) appears repeatedly at over

2817 1,000 times per million words (Conrad \& Biber, 2004). 4-word bundles, then, are less

2818 common than 3-word bundles but are not as rare as 5-and 6-word bundles, meaning an

2819 analysis of 4-word bundles results in a sufficient, but not overwhelming quantity, of 2820 data.

2821 3.5.2.2 Identifying 4-word lexical bundles

2822 Before the two classifications described above can be made, a list of lexical bundles

2823 must be extracted and identified. The first stage of this process is automated. In Sketch

2824 Engine, lexical bundles are referred to as n-grams. The function tab is Word list,

2825 where we also extracted keywords. The options that must be selected are seen in

2826 Figure 7. 


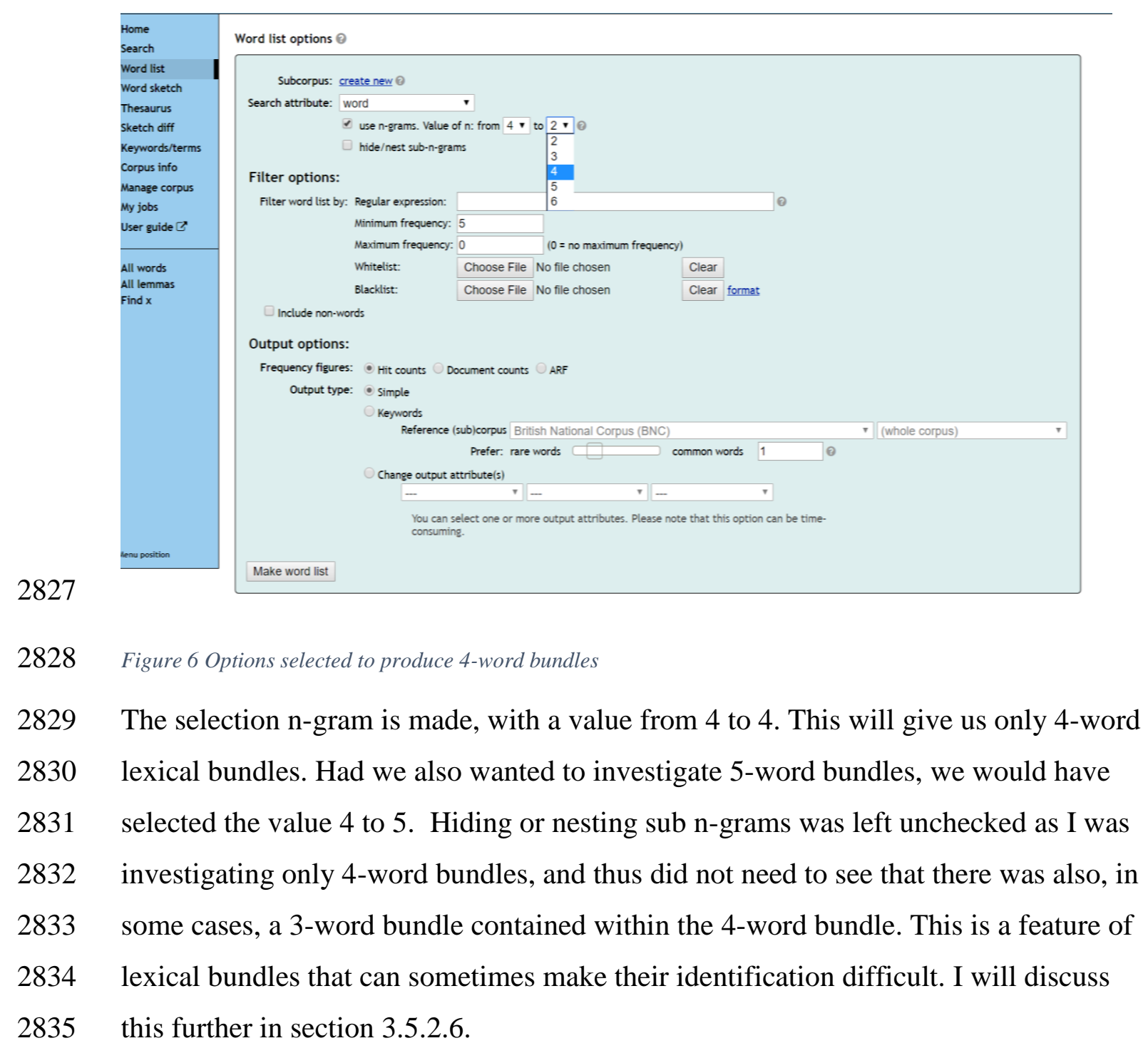

2836 There was no Blacklist or Whitelist, the Frequency figure was Hit, which counts

2837 each occurrence in a text, and the Output option was Simple. Unlike a keyword

2838 analysis, a lexical bundle analysis does not require a comparative or reference corpus

2839 but it does require that a minimum number is set, to establish a cut-off point. This will

2840 be discussed in the following section.

\section{3.5.2.3 Cut-off point and dispersion}

2842 The minimum frequency default in Sketch Engine is 5. This setting sets a cut-off

2843 point, below which the lexical bundles will be ignored. 5 is low, however, and I was

2844 concerned it would result in a lot of data. Previous studies (Cortes, 2013; Csomay,

2845 2013; Hyland, 2007) have set the minimum frequency to 20 per million words. While

284640 is also a common cut-off in similar studies (e.g Biber and Barbieri, 2007; Goźdź- 
2847 Roszkowski, 2011) it is always fairly arbitrary and much depends on the size of the

2848 corpus - mine was small - the researcher's preferences and the length of the bundle. A

2849 lower cut-off point is generally selected for the rarer, five-and si-xword bundles (e.g

2850 Cortes, 2013). It was also the case that a full description of four-word lexical bundles

2851 in procedural patient information was not the focus of this doctoral thesis but, rather,

2852 one analysis out of three. However, I examined the data extracted with the minimum

2853 setting at 20 and also at 40, finding many interesting bundles below 40 that would not

2854 be extracted if I did not lower the cut-off point. I decided, on this basis, to set the cut-

2855 off point to 20 to include these bundles.

2856 To control for individual peculiarities - that is, one or two writers favouring a

2857 certain bundle - their dispersion is also a factor. Bundles need to appear in at least five

2858 individual texts in the corpus to be included in the final list.

\section{3.5.2.4 Identifying the bundles}

2860 The first impression of the list produced, however, may be a little overwhelming, as

2861 Sketch Engine produces multiword units that are 4-words in length, though not

2862 necessarily 4-word lexical bundles. Reducing the size of the data necessitates the

2863 application of exclusion criteria, which is presented in 3.5.2.5. It also involves

2864 identifying true 4-word bundles, which, as I will now explain, is not always a

2865 straightforward procedure.

It has often been pointed out that 4-word bundles can include 3-word bundles

2867 and that 5-word bundles can contain 4-and 3-word bundles, etc. (Cortes, 2004;

2868 Hyland, 2007), which was indeed evident in my data, and the 2300+ bundles initially

2869 extracted by the corpus software also included many fragments or part bundles. It was

2870 not always straightforward to decide what was a fragment, or what could stand as a

2871 true four-word bundle, especially as some four-word bundles are better treated as a

2872 three-word bundle with a slot, e.g. during and after the. During and after can also be

2873 followed by an indefinite article or a noun with zero article, e.g. during and after an

2874 operation or during and after childbirth. The article the is dependent on the noun that

2875 follows and thus treated as a part of an optional slot. Likewise, at the top of, which is a

2876 four-word bundle. At the top of the is not, however, a fiver-word bundle, but a four-

2877 word bundle with a slot. The slot can be filled with a definite article, an indefinite 
2878 article or a possessive, e.g. at the top of the world; at the top of a tall building; at the

2879 top of his game. The decision process was time-consuming and on occasion, I turned

2880 to others for advice.

$2881 \quad 3.5 .2 .5$ Exclusion criteria

2882 The exclusion criteria applied to the list is presented below in Table 4.

2883 Table 4 Exclusion criteria applied to the extracted lexical bundles

\section{Exclusion Criteria}

1. Fragments of other bundles i.e. eat or drink any; tip of the part

2. Topic/Name specific e.g in X Plain-T; University College Central Clinic

3. Bundles with random or meaningless numbers or symbols e.g. know page 40 if;

4. Web noise e.g. at www.radio.com

5. Complete phrases e.g. do not copy this; contact us for information

The largest category excluded was the first: fragments of other bundles, along

2885 with phrases that were considered to be complete. In Chapter 2, I questioned some of

2886 the 4-word bundles Grawbowski (2015) presents in his study, as I believe that some of

2887 these bundles are examples of complete phrases or of fragments e.g. special

2888 precautions for storage; be used with caution; (the) dose should be reduced. There

2889 were many such examples in my data that needed to be removed. Structurally

2890 complete bundles that are classed as lexical bundles, such as on the other hand

2891 (Conrad \& Biber, 2005) do exist. What differentiates these from phrases that are not

2892 considered bundles is that these are formulaic in nature, unlike Grabowski's (2015)

2893 examples above.

2894 Once the exclusion criteria have been applied, and 4-word bundles identified,

2895 the remaining bundles are then classified structurally before they are assigned a

2896 discourse function. These two steps are presented below. 
2898 A lexical bundle analysis applies two classification processes to the extracted bundles, 2899 and the first of these classifications is structural. The structure of a bundle is 2900 significant: while bundles are generally not complete lexical units (with just $15 \%$ 2901 found to be complete in conversation and $5 \%$ complete in academic prose (Biber et 2902 al. (1999)), lexical bundles do possess clear, structural characteristics and different 2903 registers show preferences for different structural types.

2904 Many bundles found in spoken discourse are made up of verbs and clausal 2905 components, such as I want you to, while $90 \%$ of bundles in conversation include a 2906 verb (Conrad \& Biber, 2005). In contrast, many bundles found in written, more formal 2907 prose contain noun phrases and prepositional phrases, e.g. in the middle of. These 2908 bundles are also far more likely to contain passive structures. The taxonomy of 2909 structural categories for academic prose as presented in Biber et al. (1999) can be seen 2910 in Table 5.

2911 Table 5 Structural classification of lexical bundles in academic prose adapted. (Biber et al. 1999, p. 1015-1024)

Structure

Noun phrase with of-phrase fragments

Noun phrase with other post-modifier fragments

Prepositional phrase with embedded ofphrase fragments

Other prepositional phrase (fragment) Anticipatory it + verb phrase/adjective phrase

Passive verb + prepositional phrase fragment

\section{Examples}

the end of the, the base of the

the way in which, the relationship

between the, such a way as to

as a function of, as a result of

as in the case of, at the same time as

it is possible to, it may be necessary to is shown in figurelfig., is based on the 
Copula be + noun phrase/adjective phrase

(Verb phrase +) that-clause fragment

Verb/adjective +) to-clause fragment

Adverbial clause fragment

Pronoun/noun phrase $+b e(+\ldots)$

Other expressions may be due to, is one of the

has been shown that, that there is a

are likely to be, has been shown to be

as shown in figure/fig., as we have seen

this is not the, this did not mean that

as well as the, may or may not,

While a range of bundles types is found in both conversation and academic

2913 prose, not all bundles are used with equal frequency, in fact, there is commonly great

2914 repetition of just a few types. Conrad and Biber (2005) found that in their study

2915 comparing academic prose with conversation, just three bundle types accounted for

$291670 \%$ of the total number of 4-word bundles in conversation, and all three of these

2917 bundles included a verb. In academic prose, just two bundle types, both a noun-phrase

2918 type, represented over $60 \%$ of the 4-word bundles. These two bundle types were

2919 barely used in conversation. These marked differences, say Conrad and Biber (2005),

2920 are consistent with the differences seen between these registers at the word, clause and

2921 phrase level and are related to the communicative functions of the bundles.

2922 The second categorisation that takes place in a lexical bundle analysis is to

2923 assign discourse function, which we will now turn to.

\section{3.5.2.7 Assigning discourse function}

2925 A taxonomy of bundle meaning and purpose, first described by Cortes (2002) and

2926 later extended in Biber et al. (2003; 2004), categorised lexical bundles into three broad

2927 functions: stance, referential and discourse. An explanation of each was provided in

2928 Biber et al. (2004):

Stance bundles express attitudes or assessments of certainty that frame 
2935 Later studies expanded upon this initial taxonomy of discourse function (e.g. Cortes, 2936 2004; Cortes, 2006; Cortes, 2013) while changes to it have also been made (Hyland 2937 2008), as a result of the specific characteristics and thus discourse functions of the 2938 register being studied. In this doctoral study, I used the taxonomy set out by Biber et 2939 al. 2004 and shown in Table 6.

2940 It is necessary at this point to underline the fact that assigning a discourse 2941 function is sometimes a straightforward process as the function is clear, though 2942 sometimes it is a process that is more complex and necessitates a careful examination 2943 of the context surrounding the bundle. A single lexical bundle can have multiple 2944 functions, even, as Biber et al. (2005) point out, in a single occurrence. Take a look 2945 out can function both as a topic introducer and a directive, while the bundles the 2946 beginning of the and at the end of can function as a time reference, place reference, or 2947 text deictic reference. (p. 384). Examples of these bundle types were also found in my 2948 data and will be discussed in Chapter 6. 


\section{Functional Classification of Lexical Bundles}

\section{Stance expressions}

Express attitudes or expressions of certainty that frame some other proposition

A. Epistemic

I don't know if, I think it was

B. Attitudinal/modality stance

B1) Desire

If you want to; I like to go.

B2) Obligation/directive

you will have to; it is important to.

B3) Intention/prediction

it's going to be; I'm not going to

B4) Ability

to be able to; can be used to

\section{Discourse organisers}

Reflect relationships between prior and coming discourse

A. Topic introduction/focus

Now let's look at

B. Topic

elaboration/clarification

what this means is

\section{Referential bundles}

Make direct reference to physical or abstract entities or to the textual context itself

A. Identification/focus

that's one of the; of the things that

B. Imprecision

a little bit like; a bit more than

C. Specification of Attributes

C1) Quantity specification there's a lot of; how many of you.

C2) Tangible framing attributes

at the end of; on top of the

C3) Intangible framing attributes

the nature of the; in the case of

D. Time/place/text reference D1. Place in the department of

D2. Time

at the same time

D3. Text

in the next section; as shown in figure

D4. Multifunctional 
2960 This concludes the section on the methodology of a lexical bundle analysis. Both

2961 this and the keyword analysis already described are corpus-driven, whereas the third

2962 and final methodology used in this investigation of patient information for

2963 radiography, and which we will now turn to, is a corpus-based investigation. In a

2964 corpus-based analysis, the researcher searches the corpus for one or more linguistic

2965 items that they have decided upon a priori. For my part, I had decided to investigate

2966 the modal and semi-modal verbs used to express instructions and obligations in patient

2967 information as I was interested in how one of the primary functions of patient

2968 information, that of instructing, was linguistically realised. In short, I wanted to know

2969 more about the ways patients are told what to do in written patient information.

$2970 \quad 3.5 .3$ Modal verbs for instructions

2971 3.5.3.1 Introduction

2972 One of the functions of procedural patient information is to tell patients what to do, or

2973 how or when to do it. With radiography, some examinations necessitate that the

2974 patient does not eat or drink beforehand, while other exams require the patient to

2975 remove metal objects in the body. Patients are required to tell hospital staff if they

2976 have allergies, as these can make the use of contrast dye inadvisable. Female patients

2977 are expected to inform the staff if there is any possibility that they are pregnant. We

2978 also know that modal verbs are common to certain types of medical writing (Vihla,

2979 1999) though neither procedural or pharmaceutical patient information was discussed

2980 by Vihla (1999) (presented in Chapter 2) and, to my knowledge, there have been no

2981 studies that have investigated modal and semi-modal verbs for obligations and

2982 instructions in patient information.

2983 We currently have little idea, then, of how these words are used in these kinds of 2984 healthcare materials.

2985 The core modals are generally held to be can, could, may, might, shall, should, 2986 will, would, ought (to) and need (Downing and Locke, 1992; Quirk et al., 1985), while 2987 the category of semi-modals can include a range of items including dare to, need to, 2988 have (got) to, be able to and be going to. Semi-modals generally express a meaning 2989 that can be paraphrased with a core modal verb, and while some semi-modals are 2990 fixed expressions that cannot be marked for tense and person, e.g. had better, other 
2991 semi-modals verbs can be marked for tense and person, e.g. she has to be at work

2992 early and can also combine with certain modal verbs e.g. he should be able to and $I$

2993 might have to tell him.

2994 3.5.3.2 Types of modal meanings

2995 The main function of modal and semi-modal verbs (henceforth modals) is to express

2996 stance. (Biber, Conrad \& Leech, 2002). Modals can possess two different types of

2997 meaning, usually referred to personal (intrinsic) and logical (extrinsic) and are

2998 generally placed into one of three categories, depending on their meaning. Each

2999 category contains personal (intrinsic) and logical (meanings). The meanings are

3000 usually referred to as epistemic, deontic and dynamic, The meaning attached to

3001 modals and the names given to the types of modals will be discussed in more detail in

3002 Chapter 6, though I will now present a very brief description of these terms before I

3003 continue to describe the methodology.

3004 Epistemic modality is concerned with the speaker's attitude towards the 3005 proposition or the situation described in the proposition. This can range from an 3006 expression of doubt through to certainty. Modals commonly used to express

3007 epistemic modality include can, may and could. In table 4 in this chapter, we saw that

3008 lexical bundles can have an epistemic discourse function. Biber et al. (1992) refer to

3009 this category as permission/ability.

3010 Deontic modality is concerned with obligation, requirement and necessity.

3011 Must, have (got) to, (particularly in British English) should and need to are commonly 3012 used to express deontic modality. Lexical bundles can also have a deontic function in 3013 discourse, as we saw in Table 4 earlier in this chapter. Biber et al. (1992) refer to this 3014 category as obligation/necessity.

3015 Dynamic modality is less straightforward to characterise. Broadly speaking it 3016 refers to ability or volition - though it, unlike deontic and epistemic modality, is not 3017 subjective (Palmer, 1990, p36) which suggests that it is not inherently modal. Will, 3018 would, shall and be going to appear in this category. Biber et al. (1992) refer to this 3019 category as volition/prediction. 
3021 Deontic modality (obligation/necessity) is the category of modal that is the subject of

3022 this analysis and thus modals and semi-modals that are used exclusively as deontic

3023 modals (rather than epistemic or dynamic) in patient information are the focus.

\section{$3024 \quad$ 3.5.3.3 Methodology}

3025 The procedure for this analysis was comparatively straightforward, although there 3026 were three searches performed on three different corpora with 12 modals (listed 3027 below) the corpus of patient information; the corpus of consumer information and the 3028 corpus of general radiography. This initial frequency analysis included a range of the 3029 most common core modals and semi-modals including, though not restricted to those 3030 used to give instructions or to express obligations. The modals searched for were will, 3031 would, can, could, may, might, must, should, have to, have got to, need to, ought to. 3032 This was done in order to have an overview of the frequency of modal verb use in 3033 patient information which I could compare to what we know of the frequency in 3034 general discourse, spoken and written. Once this step had been carried out, the focus 3035 turned to those modals used for obligation, instruction and permission.

3036 How the searches were carried out in Sketch Engine will now be described.

\section{3.5.3.4 Search terms}

3038 In Sketch Engine, a search for a single item or phrase is undertaken by selecting the 3039 Search tab. The screen will offer a number of Query types: simple, lemma, phrase, 3040 word, character and CQL. A lemma will find all forms of a word, so entering examine 3041 will result in examine, examined, examining; for a search that gives you only your 3042 search term, word is the option. CQL is corpus query language, which is useful when 3043 parts of speech are being searched for (e.g. all adjectives, all conjunctions), and phrase 3044 will find examples of a sequence of tokens exactly as it is typed.

3045 The so-called Simple search is more complex than the name implies, as Sketch 3046 Engine tries to guess what it is you are looking for based on the kind of search term 3047 you have entered. If you enter a lemma, the search is a lemma search. If you enter a 3048 term which is not a lemma, a word is searched. It was the Simple search that I used 
3049 for the modal and semi-modals under investigation and the software treated my search 3050 terms as lemmas with the semi-modals and modals. This meant that the Simple search

3051 captured the changes for person and tense that took place with three of the semi-

3052 modals (need to, have to and have got to), as well as including negative forms for all 3053 modals and semi-modals.

3054 Absolute (raw) frequencies were normalised to 1 million. As I discussed in 3.4.1, 3055 normalising frequencies involves calculating the frequency of an item at 1 million 3056 words, or 100,000 or even 10,000 . This is done so that comparison between different 3057 corpora can be made. Raw frequencies, on the other hand, while reported, cannot tell 3058 us much if the corpora are of different sizes. 1 million was the figure chosen as this a 3059 Sketch Engine default.

3060 A second analysis was performed, using the same modals, but this time the 3061 corpus of consumer information, presented in 3.2.1 was used. The rationale for this 3062 has been presented in 3.2.1: patients are increasingly referred to as consumers or 3063 clients. The information produced for them may indeed be a type of consumer 3064 information, but I intuitively feel that the way patients are spoken to in procedural 3065 health information differs from the way consumers are spoken to consumer 3066 information. This may because the voice of authority in medicine is not the same as 3067 that voice of authority in a consumer advice agency. Authority, which will be 3068 discussed further in Chapter 6, is a significant factor governing the way in which 3069 obligations and instructions are presented - and perceived by the receiver. The 3070 relationships between obliger and obliged, instructor and instructed are quite different 3071 in medicine and in consumer advice. So, one way of investigating difference is to look 3072 at the way obligations and instructions are expressed in materials from both areas.

3073 Once this second analysis had been carried out, a third analysis was also 3074 conducted on the corpus of radiography, which was presented in 3.2. The latter is a 3075 corpus of 719,209 words, made up of radiography research, textbooks, handbooks and 3076 patient information. I thought it would be interesting to compare the use of modal and 3077 semi-modals verbs in this corpus as it contained a radiographer's manual, and a course 3078 book, and thus was likely full of instructions and directions. The readers of this 
materials, however, are qualified and trainee radiographers, i.e fellow medical

3080 professionals. They are not patients.

3081 The next step in my methodology was to understand how the different modals I

3082 had searched for were being used in the corpus. This was necessary in order to

3083 separate out those modals used to oblige or instruct from those with other meanings,

3084 as described earlier in 3.5.3.2. Some modals can have different meanings, it is not

3085 immediately obvious what meaning it carries and this necessitates an examination of

3086 the word in context. Can is a modal that often requires an examination of context

3087 before assigning meaning, as is should. This will be discussed in more detail in

3088 chapter 6 . Examining the modal in context involved sampling, the procedure for

3089 which I will now describe.

3090 3.5.3.5 Classifying the modals in the corpus

3091 A random sample of 100 of each modal was extracted and examined in context in the 3092 corpus. Sketch Engine offers this sampling facility, and while 250 is the default, I 3093 selected 100 to make the qualitative analysis manageable, as there were eight modals 3094 (I will use modal henceforth to include modal and semi-modal verbs) to be

3095 investigated: can, will, should, must, have to and need to. My initial investigation had 3096 resulted in zero hits for ought to, had better and have got to, so these items had been 3097 discarded. Would and could had also been removed at this point as these modals are 3098 not used to instruct or oblige.

The sampling and investigation demonstrated that can was overwhelmingly

3100 used as an epistemic or dynamic modal and will as a dynamic modal. while must, have 3101 to, should and need to were found to be always or predominantly used with a deontic 3102 meaning. These four modal verbs were then investigated in detail in the corpus and 3103 their collocational relationships were examined. These findings are presented and 3104 discussed in Chapter 6.

3105 In this chapter, I have presented the corpora used in my study of patient 3106 information for radiography, along with a detailed presentation of the contents of each 3107 corpus and the procedure, in Sketch Engine, of the building of the three corpora. I 3108 have presented, too, the three corpus methodologies used in my study. Each method is 
3109 different, and the first two are known as corpus-driven methods as the researcher does

3110 not decide what lexical item(s) will be investigated before the computer analysis is

3111 performed. The results of the analysis provide the researcher with areas for further

3112 investigation. The first method is also one of the more commonly-used approaches in

3113 corpus-assisted healthcare language studies, that of a keyword analysis. The different

3114 stages in a keyword analysis were presented which was followed by a description of a

3115 lesser-known method in studies of healthcare language, that of a lexical bundle

3116 analysis. The three different steps of the methodology have been described. I have

3117 concluded the chapter with a description of the third investigation conducted for the

3118 study: an investigation of the use of modals verbs for obligations and instructions in

3119 patient information. After an overview of the types of modal verbs according to the

3120 literature, and following a description of the initial frequency analysis carried out for

3121 comparative purposes, I have described the steps I took to identify, and then examine,

3122 four deontic modal verbs common to patient information for radiography.

3123 We now turn, in the following chapter, to a detailed presentation of the keyword

3124 extraction, carried out on my corpus of patient information for radiography.

3125

3126 


\section{4 . Keywords in Patient Information for Radiography}

2 This chapter presents the findings of a keyword analysis of patient information for

3 radiography, the first of three analyses carried out for this doctoral study. We have

4 already seen how useful keyword studies can be for revealing otherwise hidden

5 attitudes and beliefs in healthcare discourse (e.g. Harvey et al., 2008) so it seems an

6 appropriate and potentially useful analysis with which to begin my investigation of

7 patient information. After a brief definition of a keyword, I will give a brief overview

8 of the methodology, which has been presented in detail in the preceding chapter. I will

9 then present an overview of the literature; key, keyword studies (e.g. Adolphs et al.,

10 2004; Seale et al., 2006) have been discussed in detail in Chapter 2. I follow this with

11 the results and conclude with a discussion of the findings.

\subsection{Keywords}

13 The simplest definition of a keyword, as we have heard already, is that it is a

14 statistically significant lexical item (Scott,1997). The item is statistically significant

15 because it appears with unusual frequency in a given text. All keyword analyses, then,

16 involve the use of a reference corpus; a statistical analysis is carried out which

17 produces a frequency list of lexical items in the corpus under investigation when

18 compared to the reference corpus - usually, though not always, a large-scale, general

19 corpus such as the British National Corpus (BNC). In this study, as I have explained

20 in the previous chapter, I use both the BNC and a specialist corpus in order to reveal

21 key themes that may have remained hidden by using solely a general corpus. The

22 choice of reference corpus is important, says Scott and Tribble (2006; p65) but the

23 greatest concern seems to be size:

while the choice of reference corpus is important, above a certain size, the procedure throws up a robust core of KWs whichever the reference corpus used. These core KWs have largely but not exclusively to do with what the text is about; a few others are usually found which reflect some other stylistic feature. 
There have been a number of statistical tests that have been used to generate

31 keywords, though the most frequently used for keyword extraction within applied

32 linguistics are Mutual Information (MI) (Church and Hanks, 1990), Log-likelihood

33 (Dunning, 1993) and the t-test. An item may appear as a keyword with both positive

34 or negative frequency, that is, appear more or less frequently than might be expected

35 by chance. Sketch Engine uses what they refer to as Simple maths, and the calculation

36 has been presented in 3.5.1.3. Keywords are not terms or terminology, though Sketch

37 Engine, the software used in this analysis, offers the user the possibility of extracting

38 terms in addition to carrying out a keyword analysis. Terms, in Sketch Engine, are

39 two-word noun phrases that appear with greater frequency when compared to a

40 reference corpus. ESP teachers may well refer to them as noun collocations. A

41 keyword, however, can be any kind of word class and is not restricted to nouns.

A keyword analysis can provide an entry point into the data - Scott (2010)

43 refers to them not as gold nuggets, but valuable indications that the text is worth

44 mining - though in itself a keyword cannot tell us much as it does not give us any

45 information about the use of the word in the register under investigation. The

46 quantitative analysis is always followed by a qualitative investigation of selected

47 keywords in context, often referred to as KWIC. The extracted keywords are also

48 categorised semantically, which is a particularly helpful step when there is a lot of

49 data or when semantic themes are not immediately obvious. These steps have been

50 described in detail in the preceding chapter. Keywords, then, can 'reveal not only a

51 great deal about the subject matter, the "aboutness" of a particular genre, but they can

52 also specify the salient features which are functionally related to the genre (Gozdz-

53 Roszkowski, 2011, p35).

A keyword analysis has the potential to be a very useful tool to reveal more

55 about the linguistic character of patient information and the topics that the discourse

56 prioritises, though, to date, keyword studies of procedural patient information have not

57 been carried out. A handful of studies of PILs (pharmaceutical patient information)

58 have utilised the keyword method (Grabowski, 2013; 2015; 2017). Grabowski (2015)

59 is reviewed 2.5.5. In applied linguistics more broadly and in healthcare

60 communication studies more specifically the method is often used, and some of the

61 studies from the literature I will overview in the following section. Other studies, 
62 particularly those relevant to medical discourse and to this doctoral thesis, have

63 already been reviewed in detail in Chapter 2.

\subsection{Keyword studies in the literature}

65 Establishing the lexical 'aboutness' of a discipline lies behind much of the literature

66 on keywords in the field of English for Specific Purposes (ESP). An ESP keyword

67 analysis is of theoretical interest, of course, but it also has a great practical utility with

68 the development of discipline-specific wordlists and teaching materials. Lecturers and

69 teachers of discipline-specific English find that there are rarely published coursebooks

70 to fall back on (publishers do not consider ESP to be as lucrative a market as EAP,

71 English for Academic Purposes and thus are unwilling to invest (Bennett, 2010)),

72 while many educational practitioners do not have expertise in the discipline or

73 sufficient time in the programme to cover everything they feel their students require.

74 Deciding what is essential to teach, what words their students really need in order to

75 become members of the specific discourse community, becomes a priority.

76 ESP and academic word lists developed that have used the keyword approach

77 include Gilmore and Millar (2018) who look at the language of civil engineering

78 research papers; Watson-Todd (2017) who consider engineering more broadly; and

79 Pacquot (2007) who applies the criterion of keyness to the more usual criteria of

80 frequency, range and evenness of distribution for the development of an academic

81 word list. Range and frequency are the criteria more often used in word list

82 development (e.g. Coxhead, 2000; Gardner and Davies, 2014, Hsu, 2014; Mudraya,

83 2006; Wang, Liang and Ge, 2008; Ward, 2009) though Pacquot takes the view that,

84 for productive purposes, Coxhead's (2000) Academic Word List (AWL) is less useful,

85 as it excludes high-frequency words that have an important productive function in

86 academic discourse. Keyword analyses, on the other hand, do not exclude on the

87 basis of general frequency.

The concept of 'keyness' has been used in corpus-based studies which aim to

89 reveal what Baker (2004) calls 'discourses' in the language, that is, concepts 'that may

90 help to highlight the existence of types of (embedded) discourse or ideology' (Baker,

91 2004, p347). Examples of such studies include Johnson, Culpeper and Suhr (2003), 
92 who use keyword extraction to explore the discourses of political correctness (PC) in

93 three British newspapers over the course of five years, finding an overall decline in

94 the use of PC terms, while Baker (2004) looks at keywords in gay and lesbian erotic

95 fictional narratives in order to identify how identity is constructed differently in each

96 genre. Knight, Walsh and Pappagianidis (2015) investigate the discourse of e-

97 transactional language - eBay listings - using, among other tools, a keyword analysis

98 of the terms used by experienced and amateur eBay sellers.

99 Studies which aim to uncover the lexical characteristics of a particular register

100 or domain include Goźdź-Roszkowski (2011) who investigates keywords in a range of

101 legal registers and Grabowski $(2013 ; 2015)$ looks at keywords in pharmaceutical

102 registers. Grabowski (2015) is discussed in more detail in Chapter 2.

103 The introduction of the keyword technique to medical practitioners and other

104 researchers outside the field of applied linguistics has been present in a number of

105 studies that relate to healthcare discourse. Seale, Ziebland and Charteris-Black (2006)

106 and Seale and Charteris-Black (2008) use a keyword analysis to investigate the impact

107 of gender, and gender and age, respectively, on patients' experience of illness and

108 health conditions. These studies are discussed in more detail in Chapter 2. The 2006

109 study, which used a corpus of forum postings and transcribed interviews, was

110 published in the journal Social Science and Medicine, a particularly important journal

111 for healthcare communication studies. The paper introduced the concept of 'keyness'

112 to an audience of social scientists, making the case for its use alongside more

113 traditional qualitative methods.

114 Gender difference as it relates to health information was the principal focus of

115 both Seale et al. (2006) and Seale and Charteris (2008) and the two studies have

116 findings regarding gender and information-seeking behaviour that are supported in the

117 literature (e.g. Bidmon \& Terlutter, 2015; Ek, 2013; Rice, 2006; Rutten, Squiers \&

118 Hesse, 2006). These studies confirm not only that gender has an influence on how

119 people look for healthcare information, but that a difference in the type of information

120 being sought is also seen. Many studies (e.g. Ek, 2013) also show that women are

121 much more likely than men to engage in health information seeking. As Ek (2013) 
122 says 'When it comes to health, women seem to be more engaged, more involved,

123 more attentive and apparently better-informed decision-makers.' (p742).

124 There is nothing fixed about the linguistic performance of gender (Seale and 125 Charteris-Black, 2008), but if men and women tend to look for different information 126 and focus on different aspects of health, illness and treatment, it seems reasonable to 127 consider whether existing patient information, such as that in the corpus used in this 128 thesis, is appropriate to these different needs. A keyword analysis can help reveal 129 some of the information priorities in patient information for radiography which can 130 help us answer the former question.

131 Seale and Charteris-Black (2008) also found that older men, in particular, like

132 to reference medical experts and specialists such as radiologists, oncologists and

133 consultants while it is woman of all ages who are much more likely to talk about, and

134 talk with, nurses. The important role of nurses - and radiographers and technologists -

135 in a patient's experience of radiography does not seem to be reflected in their

136 appearance in patient information, however. This will be discussed in more detail in $137 \quad 4.5 .3 .2$.

138 Now let us turn briefly to the methodological steps taken in this keyword 139 study. Full details of the methodology have been presented in Chapter 3.

\section{$140 \quad 4.3$ Methodology}

141 A full description of the keyword methodology has been presented in Chapter 3. There 142 were two separate keyword extractions performed, one with the BNC $(96,134,547$

143 words) and one with the corpus of radiography (719,209 words).

144 For the comparison with the BNC, the minimum frequency was set at 5, the 145 default value in Sketch Engine. For the analysis with the corpus of radiography as a 146 reference corpus, the minimum frequency was also set at 5 . With the setting at 5 , the 147 number of keywords extracted with the BNC as reference corpus was 991 . When the 148 corpus of Radiography was used, the number of keywords was 965 . These numbers 149 are pre-data cleaning which I explain in the following section. 
150 The data cleaning (data cleaning refers to the removal of data - lexical items 151 that will not be considered in the final list) focused only on the first 50 items, as only

152 these items were being evaluated in this study. Data cleaning resulted in a number of

153 items being removed: three URLs or part domain names and two professional

154 association acronyms. Acronyms that related to medical procedures or radiographic

155 modalities and thus were part of the content were left (e.g. DCIS - ductal carcinoma

156 in situ; CT - computed tomography or MRI - magnetic resonance imaging). These

157 acronyms are considered part of the content as they are used in speech and writing to 158 name procedures, modalities and examinations, and while they may be spelt out once

159 in the text, they are then often used as acronyms without a definition. The results of

160 the analyses are presented in the next section.

$161 \quad 4.4$ Results

$162 \quad$ 4.4.1 Keyword lists

163 With the minimum setting at 5, the resulting list of keywords with the BNC as a

164 reference corpus totalled 991 . With the radiography corpus as a reference corpus, with

165 all parameters untouched, the total was 965 . The first 50 keywords of both analyses

166 can be seen in Tables 7 and 8 .

167 The Freq column refers to the raw frequency of the token in the corpus, which,

168 as we have seen, means the number of individual occurrences of the item in the

169 corpus. The Freq/mil is the adjusted frequency, per million words. This as we have

170 seen, is the default setting in Sketch Engine. The score in the final column is the

171 keyness score. The keyness score is the statistical calculation of the significance of the

172 lexical item, though as we have seen in 3.5.1.8, the precise placings of the keywords

173 do not mean that they are arranged in order of importance but in order of keyness. The

174 first keyword $C T$ is not necessarily any more significant than the 3rd (radiation) or the

$17520^{\text {th }}$ keyword (web) as much depends on the frequency of these words in both corpora.

176 A semantic categorisation and an investigation of the lexical item in the context of the

177 corpus are necessary steps to understand the significance of a keyword. 


\begin{tabular}{|c|c|c|c|c|c|}
\hline Keyword & Freq & Freq/mill & Freq_ref & Ref/mill & Score \\
\hline & \multicolumn{2}{|c|}{ Patient Information } & $B N C$ & & \\
\hline MRI & 1368 & 2929.7 & 102 & 0.9 & 1536.1 \\
\hline radiologist & 821 & 1758.2 & 26 & 0.2 & 1428.6 \\
\hline $\mathrm{ct}$ & 1657 & 3548.6 & 184 & 1.6 & 1345.7 \\
\hline radiology & 797 & 1706.8 & 46 & 0.4 & 1211.7 \\
\hline ultrasound & 1106 & 2368.6 & 202 & 1.8 & 846.9 \\
\hline technologist & 522 & 1117.9 & 38 & 0.3 & 836.1 \\
\hline tumor & 370 & 792.4 & 21 & 0.2 & 668.4 \\
\hline imaging & 1447 & 3098.8 & 441 & 3.9 & 629.4 \\
\hline tumors & 289 & 618.9 & 4 & 0 & 598.6 \\
\hline interventional & 276 & 591.1 & 9 & 0.1 & 548.2 \\
\hline x-ray & 1311 & 2807.6 & 623 & 5.5 & 429.1 \\
\hline catheter & 417 & 893 & 127 & 1.1 & 419.6 \\
\hline transducer & 291 & 623.2 & 61 & 0.5 & 404.5 \\
\hline physician & 986 & 2111.6 & 493 & 4.4 & 392.1 \\
\hline medications & 252 & 539.7 & 56 & 0.5 & 360.8 \\
\hline radiological & 416 & 890.9 & 171 & 1.5 & 353.6 \\
\hline scan & 1090 & 2334.3 & 669 & 6 & 335.8 \\
\hline
\end{tabular}




\begin{tabular}{|c|c|c|c|c|c|}
\hline Keyword & Freq & Freq/mill & Freq_ref & Ref/mill & Score \\
\hline & \multicolumn{2}{|c|}{ Patient Information } & $B N C$ & & \\
\hline $\mathrm{x}$-rays & 586 & 1255 & 327 & 2.9 & 321.2 \\
\hline radiologists & 154 & 329.8 & 15 & 0.1 & 291.8 \\
\hline radiologic & 138 & 295.5 & 2 & 0 & 291.3 \\
\hline angiography & 184 & 394 & 42 & 0.4 & 287.5 \\
\hline tomography & 283 & 606.1 & 125 & 1.1 & 287.3 \\
\hline anesthesia & 131 & 280.5 & 5 & 0 & 269.5 \\
\hline radiotracer & 125 & 267.7 & 0 & 0 & 268.7 \\
\hline copyrighted & 131 & 280.5 & 6 & 0.1 & 267.3 \\
\hline sedation & 224 & 479.7 & 93 & 0.8 & 263.0 \\
\hline radiographer & 133 & 284.8 & 11 & 0.1 & 260.3 \\
\hline mammography & 133 & 284.8 & 11 & 0.1 & 260.3 \\
\hline radiofrequency & 124 & 265.6 & 3 & 0 & 259.6 \\
\hline embolization & 116 & 248.4 & 0 & 0 & 249.4 \\
\hline exam & 948 & 2030.2 & 868 & 7.7 & 232.8 \\
\hline noninvasive & 105 & 224.9 & 0 & 0 & 225.9 \\
\hline copyright & 1015 & 2173.7 & 999 & 8.9 & 219.8 \\
\hline download & 130 & 278.4 & 33 & 0.3 & 216.0 \\
\hline physicians & 404 & 865.2 & 363 & 3.2 & 204.7 \\
\hline
\end{tabular}




\begin{tabular}{|c|c|c|c|c|c|}
\hline Keyword & Freq & Freq/mill & Freq_ref & Ref/mill & Score \\
\hline & \multicolumn{2}{|c|}{ Patient Information } & $B N C$ & & \\
\hline prostate & 218 & 466.9 & 161 & 1.4 & 192.3 \\
\hline ionizing & 105 & 224.9 & 22 & 0.2 & 188.9 \\
\hline radiation & 1416 & 3032.5 & 1713 & 15.2 & 186.7 \\
\hline web & 528 & 1130.7 & 572 & 5.1 & 185.8 \\
\hline anesthetic & 86 & 184.2 & 0 & 0 & 185.2 \\
\hline brachytherapy & 85 & 182 & 0 & 0 & 183 \\
\hline scanner & 366 & 783.8 & 370 & 3.3 & 182.8 \\
\hline carotid & 100 & 214.2 & 28 & 0.2 & 172.2 \\
\hline ablation & 119 & 254.8 & 55 & 0.5 & 171.8 \\
\hline intravenous & 339 & 726 & 367 & 3.3 & 170.4 \\
\hline scans & 190 & 406.9 & 163 & 1.5 & 166.4 \\
\hline reviewed & 1077 & 2306.5 & 1466 & 13 & 164.2 \\
\hline jewelry & 83 & 177.7 & 11 & 0.1 & 162.8 \\
\hline clots & 99 & 212 & 39 & 0.3 & 158.1 \\
\hline barium & 171 & 366.2 & 153 & 1.4 & 155.5 \\
\hline
\end{tabular}


180 In Table 8 below, we see the top 50 keywords when using the general Radiography

181 corpus as a reference corpus.

182 Table 8 Top 50 keywords using the general radiography corpus

\begin{tabular}{|c|c|c|c|c|c|}
\hline Keyword & Freq & Freq/mill & Freq/ref & Ref/mill & Score \\
\hline & \multicolumn{3}{|c|}{ Patient Information } & \multicolumn{2}{|c|}{ Radiography } \\
\hline leaflet & 327 & 700.3 & 1 & 0.9 & 369.3 \\
\hline copied & 147 & 314.8 & 0 & 0 & 315.8 \\
\hline warranty & 130 & 278.4 & 0 & 0 & 279.4 \\
\hline warranties & 130 & 278.4 & 0 & 0 & 279.4 \\
\hline radiotracer & 125 & 267.7 & 0 & 0 & 268.7 \\
\hline copyright & 1015 & 2173.7 & 8 & 7.2 & 265.5 \\
\hline interprets & 94 & 201.3 & 0 & 0 & 202.3 \\
\hline breastfeeding & 87 & 186.3 & 0 & 0 & 187.3 \\
\hline illustrative & 131 & 280.5 & 1 & 0.9 & 148.3 \\
\hline copyrighted & 131 & 280.5 & 1 & 0.9 & 148.3 \\
\hline representations & 130 & 278.4 & 1 & 0.9 & 147.1 \\
\hline download & 130 & 278.4 & 1 & 0.9 & 147.1 \\
\hline fibroid & 59 & 126.4 & 0 & 0 & 127.4 \\
\hline cryotherapy & 57 & 122.1 & 0 & 0 & 123.1 \\
\hline dye & 156 & 334.1 & 2 & 1.8 & 119.8 \\
\hline thrombolysis & 52 & 111.4 & 0 & 0 & 112.4 \\
\hline
\end{tabular}




\begin{tabular}{|c|c|c|c|c|c|}
\hline Keyword & Freq & Freq/mill & Freq/ref & Ref/mill & Score \\
\hline & \multicolumn{3}{|c|}{ Patient Information } & \multicolumn{2}{|c|}{ Radiography } \\
\hline fibroids & 49 & 104.9 & 0 & 0 & 105.9 \\
\hline prick & 47 & 100.7 & 0 & 0 & 101.7 \\
\hline resume & 89 & 190.6 & 1 & 0.9 & 100.9 \\
\hline pictures & 256 & 548.2 & 5 & 4.5 & 100 \\
\hline prohibited & 130 & 278.4 & 2 & 1.8 & 99.9 \\
\hline disclaimer & 130 & 278.4 & 2 & 1.8 & 99.9 \\
\hline loose-fitting & 46 & 98.5 & 0 & 0 & 99.5 \\
\hline pals & 43 & 92.1 & 0 & 0 & 93.1 \\
\hline web & 528 & 1130.7 & 13 & 11.7 & 89.2 \\
\hline warned & 38 & 81.4 & 0 & 0 & 82.4 \\
\hline sonar & 38 & 81.4 & 0 & 0 & 82.4 \\
\hline inaudible & 36 & 77.1 & 0 & 0 & 78.1 \\
\hline enterography & 36 & 77.1 & 0 & 0 & 78.1 \\
\hline transvaginal & 32 & 68.5 & 0 & 0 & 69.5 \\
\hline television-like & 32 & 68.5 & 0 & 0 & 69.5 \\
\hline piercings & 31 & 66.4 & 0 & 0 & 67.4 \\
\hline chemoembolization & 31 & 66.4 & 0 & 0 & 67.4 \\
\hline vertebroplasty & 30 & 64.2 & 0 & 0 & 65.2 \\
\hline
\end{tabular}




\begin{tabular}{|c|c|c|c|c|c|}
\hline Keyword & Freq & Freq/mill & Freq/ref & Ref/mill & Score \\
\hline & \multicolumn{3}{|c|}{ Patient Information } & \multicolumn{2}{|c|}{ Radiography } \\
\hline kyphoplasty & 29 & 62.1 & 0 & 0 & 63.1 \\
\hline assure & 134 & 287 & 4 & 3.6 & 62.7 \\
\hline numb & 54 & 115.6 & 1 & 0.9 & 61.4 \\
\hline loaf & 28 & 60 & 0 & 0 & 61 \\
\hline up-to-date & 130 & 278.4 & 4 & 3.6 & 60.8 \\
\hline sonohysterography & 27 & 57.8 & 0 & 0 & 58.8 \\
\hline transrectal & 26 & 55.7 & 0 & 0 & 56.7 \\
\hline thinners & 26 & 55.7 & 0 & 0 & 56.7 \\
\hline magnets & 26 & 55.7 & 0 & 0 & 56.7 \\
\hline box-like & 26 & 55.7 & 0 & 0 & 56.7 \\
\hline outweighs & 48 & 102.8 & 1 & 0.9 & 54.7 \\
\hline urogenital & 25 & 53.5 & 0 & 0 & 54.5 \\
\hline breastfeed & 25 & 53.5 & 0 & 0 & 54.5 \\
\hline please & 758 & 1623.3 & 33 & 29.7 & 53 \\
\hline aneurysms & 68 & 145.6 & 2 & 1.8 & 52.4 \\
\hline sting & 24 & 51.4 & 0 & 0 & 52.4 \\
\hline
\end{tabular}


184 Organising the keywords into semantic categories was the next step. As I have

185 explained in the preceding chapter, semantic classification helps reveal the primary

186 concerns of the register, as themes can be more readily uncovered. I selected

187 categories for each item in the top 50 in both lists, basing my decision on the broader

188 meanings of each word and, as I have explained in detail in 3.5.1.9, using the Sketch

189 Engine facility Word Sketch, along with examination of the item in the context of the

190 corpus. Word Sketch gives the user collocational information, both lexical and

191 grammatical, about a word. This collocational information references the corpus, it

192 should be noted.

Both analyses resulted in 10 categories. Seven of these categories were shared

194 and three categories unique. There were no items that appeared in more than one

195 category. Examination of each word in its context revealed that two items, up-to-date

196 and reviewed, were being used in a way that was not immediately obvious. Both items

197 were moved from the category Information to the category Legal, after inspection in

198 the corpus. All examples of both reviewed and up-to-date, while referring to the

199 information contained in the leaflet, were used in formulaic phrases relating to a legal

200 disclaimer.

201 As expected, the analysis with the BNC produced three categories that were

202 more specific to radiography and radiographic procedures: Medical

203 instrument/equipment; Radiographic modality and Medical exam/procedure.

204 In contrast, when the Radiography corpus was used as a reference corpus,

205 there were no keywords in the top 50 from these three categories, but three new

206 categories were created: Other: Body; Other: NHS and General. Other: Body contains

207 items that could not be classed as body parts, but were related, nonetheless, to the

208 human body, while Other: NHS contains just one item, an acronym that references a

209 service offered within the NHS called PALS - the Patient and Liaison Advisory

210 Service. General contains any items that were general in meaning.

211 The categories in common were Medical professionals; Body part or organ;

212 Treatment or therapy; Disease or condition; Radiography or radiotherapy; Information 
213 and Legal, though as we see in Table 9, the majority of the words appearing in these

214 categories were unique to the analysis. Words in common are bolded

215 Table 9 Semantic classification of keywords

BNC as ref corpus $\quad$ Category $\quad$ Radiography ref corpus

radiologist, radiologists,

physician, radiographer, Medical

physicians,

professional

technologist,

urogenital; breastfeed;

clot, prostate, carotid, Body

breastfeeding, transvaginal,

transrectal

medications,

embolization,

noninvasive,

Treatment/therapy

brachytherapy, ablation,

interventional

cryotherapy, kyphoplasty, chemoembolization, vertebroplasty, thinners, thrombolysis

catheter, transducer,

Medical

scanner

instrument or

equipment

CT, x-ray, x-rays,

Radiographic

Inaudible, sonar, loaf, magnets,

ultrasound, MRI, modality box-like, television-like

tomography, 


\begin{tabular}{lll} 
BNC as ref corpus & Category & Radiography ref corpus \\
\hline $\begin{array}{l}\text { scan, angiography, } \\
\text { mammography, exam, }\end{array}$ & Medical exam & enterography, sonohysterography, \\
$\begin{array}{l}\text { scans, barium } \\
\text { tumor, tumors }\end{array}$ & Disease/condition & fibroid, fibroids, aneurysms, \\
radiology, imaging, & & \\
radiological, radiologic, \\
radiotracer, \\
radiofrequency, \\
$\begin{array}{l}\text { Radiography and } \\
\text { ionizing, radiation, }\end{array}$
\end{tabular}

copyrighted, copyright Legal

reviewed

anesthesia, sedation, anesthetic, intravenous

Medical: other

Other:

NHS/Healthcare

system

jewelry

General copied, warranty, warranties,

copyright, interprets, illustrative, copyrighted, representations, prohibited, disclaimer, up-to-date, warned, assure

numb, sting, prick

\section{PALS}

please, resume, outweighs, loose-

fitting, piercings 
216 The results of the semantic classification seen in table form gives an insight

217 into the most important themes in patient information. We see that the technology of

218 radiography is prominent, along with a variety of radiographic examinations. The

219 professionals who work in radiology feature, as are treatment and therapy. An

220 unexpected category is Legal. Without examining these words in the corpus, however,

221 we cannot say much about how they are being used in the patient information. The

222 results of these investigations will be presented in more detail in 4.5 .3 , though before

223 this, and in the next section, I will present results relating to the class of words, i.e.

224 parts of speech appearing in the keyword list.

\section{4.4.3 Word type or parts of speech}

226 We know that spoken registers are very often fundamentally different from written

227 registers in their use of grammatical and lexical features (Biber, Conrad, Reppen,

228 Byrd \& Helt, 2002). There is more repetition in spoken discourse, with a lower lexical

229 density than in written language (McCarthy, 1998). We have seen, too, in chapter 3 ,

230 that the structure of lexical bundles differs markedly between conversation and

231 academic discourse (Biber et al. 1999; Conrad \& Biber, 2004), with academic

232 discourse preferring noun phrases, prepositional phrases and passive structures, while

233 conversation tends to make use of clauses, which centre around a verb. A full list of

234 the common structure-types in academic discourse described by Biber et al. (1999)

235 can be found in table 3 in section 3.5.2.6.

236 As I explained in 3.5.1.2, keywords can be lexical words, grammatical words

237 or a combination of the two. The so-call smoothing parameter in Sketch Engine allows

238 a user to decide whether they want more or less common words (common words are

239 likely to include a high proportion of grammatical words) in their keyword list. Even

240 with a high proportion of content words - which is what I wanted in this doctoral study

241 - there will be different proportions of nouns, verbs, adjectives and adverbs. What

242 class of words appear frequently may give us an insight into the structure of patient

243 information, e.g. whether patient information likes naming things, and thus nouns will

244 predominate, whether the focus is on doing and action, in which case there might be a

245 large number of verbs or whether describing things is particularly prominent, in which

246 case we might see a higher frequency adjectives and adverbs. 
In my data, sometimes a word was used as a noun and a verb: in these few

248 cases (e.g. drink; visit) the most predominant use in the corpus was the one selected. I

249 could have marked these words as belonging to both categories, but in both cases, the

250 verb was overwhelmingly preferred, so I chose to list it solely in this category in the

251 patient information. In the section that follows, I will present the results of the word

252 classes of the keywords.

253 4.4.3.1 Nouns

254 The keywords extracted in the first analysis, with the BNC as a reference corpus, are 255 predominantly nouns. There are 41 nouns of various types (singular, plural,

256 uncountable). The categories with the most nouns are Medical professional with 6;

257 Treatment or therapy with 6. Radiographic modalities are also very present with 6

258 nouns that name the different radiographic technologies, while a further 5 nouns relate

259 to the fields of radiology, radiography and radiotherapy. Examinations that use

260 radiography account for another 6 nouns.

261 There is a greater variety of word type in the second analysis, with the corpus of

262 Radiography as the reference corpus. The results included 25 nouns of various types

263 (singular, plural and uncountable), around half of the number extracted using the BNC

264 as a reference corpus. There are only two nouns in common: web and copyright. As

265 copyright can be a noun, adjective and verb, I used Word Sketch to give me the parts

266 of speech information I needed: $90 \%$ of the uses of copyright in the corpus are as a 267 noun.

268 4.4.3.2 Verbs

269 There are 2 verbs included in the first 50 keywords with the BNC as reference corpus:

270 download and reviewed. This is likely to be explained by what has been reported in

271 the preceding section: the more noun-dense a text is, the correspondingly less verb-

272 dense (and less pronoun-dense) it will be (Biber, 1988).

273 The second analysis with the radiography corpus does include verbs, however:

274 there are 10 verbs (in all forms) in the list of keywords: copied, reviewed, interprets, 
275 assure, resume, warned, breastfeeding, breastfeed, outweigh and download. The first

276 four verbs are categorised as legal verbs.

278 There were 7 adjectives in the first 50 keywords with the BNC as a reference corpus.

279 In contrast, the analysis with the radiography corpus included 13 adjectives:

280 informational, illustrative, up-to-date, copyrighted, prohibited, loose-fitting, numb,

281 inaudible, box-like, television-like, transrectal, transvaginal and urogenital.

282 Four of these seemed to relate to the patient information itself: informational,

283 illustrative, up-to-date and reviewed though on closer inspection the first three were

284 used in a legal disclaimer. Two further adjectives related clearly to legal issues:

285 copyrighted, prohibited. This was by far the largest category in the second keyword

286 analysis using the radiography reference corpus. It will be discussed in more detail in

287 section 4.5.3. The distribution of the different classes of word in both keyword lists

288 (with BNC as reference corpus and with the radiography corpus, can be seen in Table

28910 below.

290 Table 10 Distribution by word class in keyword lists

\section{Word Class BNC Keyword list Radiography Keyword list}

\begin{tabular}{lcc}
\hline Noun (all) & 41 & 25 \\
Verb (all) & 2 & 10 \\
Adjective (all) & 7 & 13 \\
Other & 0 & 2 \\
Total & $\mathbf{5 0}$ & $\mathbf{5 0}$ \\
\hline
\end{tabular}

The different range of word class seen in Table 9 above suggests that using a

292 specialised corpus can indeed give valuable insights into the lexical characteristics of

293 patient information that using a general corpus cannot. When the BNC was used, the

294 technical and medical nouns that are specific to radiography predominated; they are

295 classed as keywords because they are very rare in general English (and in any other 
296 variety of English except radiography and radiology). The second extraction produced

297 keywords that, while sharing many of the same categories with the keywords

298 extracted in the first analysis, were more varied in meaning and word class.

\section{4.4.4 First 10 Keywords}

300 We can also present a snapshot of the results of the keyword extraction by focusing on

301 the first 10 keywords from each extraction. This snapshot gives us some important

302 information at a glance about the prevalence of certain ideas in the corpus of patient

303 information. I have listed these keywords again for convenience below in Table 11.

304 Table 11 1st 10 keywords with both BNC and radiography reference corpora

\begin{tabular}{ll}
\hline 1st 10 with BNC & 1st 10 with Radiography corpus \\
\hline & leaflet \\
x-ray & copied \\
catheter & warranty \\
transducer & warranties \\
physician & radiotracer \\
medications & copyright \\
radiological & interprets \\
scan & breastfeeding \\
x-rays & illustrative \\
radiologists & copyrighted \\
radiologic &
\end{tabular}

It is worth remembering at this point that the relevance or otherwise of a

306 statistically-significant keyword cannot be assumed by its position in a keyword list.

307 As Scott (2010) says, "the greater the number of keywords extracted, the greater the

308 possibility that the inclusion of some is based on statistical chance'. Nonetheless,

309 focusing on just 10 words may make it easier to see themes emerging. 
In Table 10, we can see that words relating to radiography and medicine

311 predominate in the extraction with the BNC: $x$-ray, $x$-rays and scan are present (scan

312 refers to a CT or MRI exam (the machines themselves are scanners; the exam a

313 scan)). There are two medical professionals: the radiologist, who is a specialist doctor

314 and not to be confused with radiographer, the person who performs the scan or x-ray,

315 and physician, the American term for doctor. Catheter, transducer and medications

316 also appear: that these terms are considered key is not overly surprising as one of the

317 primary purposes of patient information is to explain radiologic procedures such as $\mathrm{X}$ -

318 ray, ultrasound and CT. These diagnostic tests also happen to be the most commonly

319 performed radiographic tests in the UK, in that order. ${ }^{4} \mathrm{MRI}$ is performed just a little

320 less frequently than CT (in the UK) (0.26 million compared to 0.38 million in March

$3212016)$ and appears as the $11^{\text {th }}$ keyword in this analysis.

322 Turning now to the extraction with the Radiography corpus, there are no words

323 in common. The first word is leaflet. There is also a reference to breastfeeding, which

324 relates to the safety of radiation-examinations for breastfeeding mothers and

325 radiotracer, a reference to nuclear radiation. The remaining items in this short list all

326 relate to the Legal category, which it transpires contains more keywords than any

327 other category in the extraction with the Radiography corpus. The significance of this

328 will be discussed in 4.6.1 below.

329 As we know, however, a keyword in a list tells us little, either about its use in a

330 register or about its significance - its 'keyness' - in the register. It is this qualitative

331 investigation of the words in our semantic categories, which includes collocation

332 information, that gives us a picture of a keyword's significance in a register. It is these

333 investigations, and their findings, that are the focus of the Discussion section that

334 follows.

\footnotetext{
${ }^{4}$ https://www.england.nhs.uk/statistics/wp-content/uploads/sites/2/2015/08/Provisional-MonthlyDiagnostic-Imaging-Dataset-Statistics-2016-07-21.pdf
} 
336 The analysis with the BNC as the reference corpus results in many keywords, as we

337 saw in Table 8, that are classified as medical and technical words, with many items

338 that are strictly related to radiography, or to healthcare. Modalities, i.e. the type of

339 radiographic technology; machines, examinations, along with some medical

340 professionals and some treatment options.

341 When the analysis is conducted with the Radiography corpus as the reference

342 corpus, the resulting categories are similar - there are 10 categories in common - but

343 the keywords are usually different. In fact, there are only 3 keywords, out of 50, in

344 common: reviewed; commercial, copyright. The semantic categorisation of all 50

345 keywords from both analyses has been presented earlier in Table 10. The finding that

346 the categories are shared but the words they contain are different confirms, I feel, both

347 the utility of the keyword method in highlighting the themes and areas of interest in a

348 register, but also the value of conducting a second keyword extraction, in my case

349 with the Radiography corpus, as it appears to have revealed a semantically richer

350 variety of keywords.

351 The categories and some of the keywords that they contain will now be

352 discussed in detail below. I have chosen the categories and keywords that seemed to

353 me to represent particularly interesting implications for the register under

354 investigation, that of patient information.

355 4.5.1 Legal

356 In the analysis with the BNC, there were three items in this category: copyright,

357 copyrighted and reviewed, which always appear in a disclaimer. These words both

358 reference and delimit, what can be done with the information.

359 (1) Permission is granted to modify and/or reproduce this leaflet for purposes

360 relating to the improvement of healthcare, provided that the source is

$361 \quad$ acknowledged and that none of the material is used for commercial gain.

362 There was just one example (out of 130) of copyrighted being used in the negative,

363 and encouragement given to reproduce the information: 
(2) This publication is not copyrighted. The Clearinghouse encourages users of this

reference corpus, containing 12 words: copied, warranty, warranties, copyright, copyrighted, prohibited, disclaimer, representations, assure, illustrative, reviewed, and up-to-date. The difference in the variety of English was stark: $90 \%$ of occurrences of the words in the legal category were found in the US-sourced information. While some of these words are overtly legal, e.g. warranty, others, e.g. up-to-date or illustrative are general terms used with a legal meaning in this context: conclusions or make diagnoses by comparing these images to other medical images, particularly your own.

376 Many of these legal words appeared together with other legal keywords and thus were very formulaic in nature:

(4) However, it is not possible to assure that this Web site contains complete, upto-date information on any particular subject. Therefore, ACR and RSNA make no representations or warranties about the suitability of this information for use for any particular purpose.

sourced in the US. The appearance of legal disclaimers in healthcare information may

385 that does not necessitate paying for treatment via private insurance), though given that

386 half of all medical malpractice claims in the US relate to diagnosis ${ }^{5}$ and treatment, and

387 that malpractice pay-outs were valued at more than $\$ 3.7$ billion in 2013 (and growing

388 year on year) (ASC Communications, 2018), it is understandable that the producers of

389 patient information wish to protect themselves. The legal references in my corpus

\footnotetext{
${ }^{5}$ https://www.beckershospitalreview.com/legal-regulatory-issues/medical-malpractice-in-america-15latest-statistics.html
} 
390 related not only to the advice and information itself but also to its commercial

391 distribution.

392 In my data, encouraging communication with the patient's provider is a feature

393 of many of the disclaimers that appeared at the end of the information, even when the

394 word disclaimer is not used (as it is in much of the US information, suggesting that its

395 inclusion is necessary by law). Patients are urged not to use the information in the

396 leaflet to make treatment decisions and that the information is not intended to replace

397 a visit to a doctor.

398 (5) This leaflet tells you about the procedure known as nephrostogram. It explains

399 what is involved and what the possible risks are. It is not meant to be a substitute for

400 informed discussion between you and your doctor, but can act as a starting point for

401 such a discussion.

402 The encouragement to communicate with a healthcare provider may be falling 403 on deaf ears, however, as studies suggest (e.g. Diaz, Griffith, Ng, Reinert, Friedmann

404 \& Moulton, 2002; Silver, 2015; Yeo, 2016) that more than 50\% of patients who use

405 healthcare information found online do not discuss the information with their

406 provider.

407 Other studies find that accessing healthcare information online may even, in 408 some cases, even lead to fewer subsequent doctor's visits (Shim, Ailshire, Zelinski \& 409 Crimmins, 2018). While many studies concern themselves with online information, 410 known hereafter as e-health (Eysenbach, 2001), their findings may also be relevant to 411 the kind of information that hospitals and medical services produce, much of which

412 ends up online on the hospital website, in addition to being made available as a printed

413 leaflet. All of the documents contained in my corpus were available to be printed off

414 at home, and many documents seemed to replicate what would also be available in a

415 hospital or GP surgery.

416 In a relatively early study on e-health, Diaz et al., (2002) found that $59 \%$ of the 417 respondents did not discuss information found online with their doctors. Patients who 418 did discuss internet information with their medical provider rated online information 419 as more reliable than those who did not, and between $50-60 \%$ of all patients surveyed 
420 felt that information recommended by a doctor or on a hospital site was more

421 trustworthy than that from a non-profit organisation (30\%) or a site sponsored by a

422 pharmaceutical company (16\%) (p183). 60\% of the respondents in this early study

423 rated online information as good as, or even better than that from their doctor.

424 A more recent study (Waring, McManus, Amante, Darling \& Kiefe, 2018)

425 found an even lower percentage of patients, just over $33 \%$, discussed their online

426 health information with their providers, suggesting that as we become more familiar

427 with the internet, we may use information more independently and feel less inclined to

428 share it with healthcare providers. This, and other studies, find that a higher education

429 level, higher socioeconomic status and frequency of online-information seeking is

430 associated with provider discussions (Graffigna, 2017; Waring et al., 2018).

431 Expressed another way, the less educated you are, the lower your income and the less

432 familiar you are with e-health searching, the less likely you are to discuss any

433 information you may find online with your healthcare provider, in spite of the

434 encouragement in patient information to do so. Clearly, in this scenario, there is a

435 negative impact on shared-decision making which is defined as 'an approach where

436 clinicians and patients share the best available evidence when faced with the task of

437 making decisions, and where patients are supported to consider options, to achieve

438 informed preferences' (Elwyn et al., 2010).

439 What are the factors that explain what appears to be an increasing number of 440 patients choosing not to discuss information with their doctor? It seems the reasons

441 behind this decision are complex and varied but can include feeling embarrassed,

442 feeling that the doctor does not want to hear about it, believing there is no need to talk

443 about it and simply forgetting (Silver, 2015). As we have already seen, the

444 availability of printed or digital patient information is central to the policy of shared

445 decision making; it is ironic, then, that the very people who would benefit from

446 discussing information with their doctors are less likely to do so.

447 Let us return for a moment to the characteristics of 'legalese'. Could the 448 language of legal disclaimers be off-putting for readers? My personal response to the

449 US legal disclaimer was negative, though this may be because I am not used to seeing 450 overtly legal text in healthcare information. However, the differences between the 
insurance-based medical systems in the US and the NHS in UK, free-at-the-point-ofcare, may lead some readers to the conclusion that legal disclaimers are unlikely to be

453 a feature of UK written patient information. My research tells me that disclaimers are

454 included if the writer considers it necessary in the UK, but there is currently no

455 obligation to do so.

A 2018 post in the 'Patient Information Forum', a respected association for professional patient information writers in the UK, in response to a question regarding the necessity of writing a disclaimer on patient materials, was:

I do include disclaimers when we reference a third-party website as we have no control over the content they contain. In our own information however, we don't include formal disclaimers but do often state that the leaflet isn't meant to replace discussion with a healthcare professional and also include wording to encourage patients to contact us (or NHS24 or their GP if appropriate) if they have questions and concerns.

(Thomson, Patient Information Forum, 2018)

The writer worked for the NHS. Another respondent, also working for an NHS

487 The passage above (which I must make clear is not included in my corpus of patient 488 information) contains a number of the keywords that were also extracted in my 
489 analysis of my corpus: treatment, healthcare, web, up-to-date, leaflet and reviewed.

490 The legal disclaimer drives home the point regarding advice and involvement from a

491 professional, as we see in the following four sentences that express the idea:

492 (6) ...the information is designed to complement the advice of professional

$493 \quad$ healthcare staff.

494 (7) The leaflets should not be used on their own without appropriate medical 495 advice.

496 (8) Procedures described should only be undertaken after you have received 497 training by healthcare professionals...

498 (9) ...we must emphasise the need for you to seek advice from a healthcare 499 professional about your particular situation.

500 There are also two sentences that are more legal in tone and thus more formulaic:

501 (10) ...the Trust will not be liable for injury or loss incurred as a result of actions $502 \quad$ taken by individuals after reading the materials

503 (11) All information is copyright and must not be adapted or reproduced without 504 medical provider (spoken or written) is clearly of great relevance in the NHS: nearly

507 half of the sentences in the disclaimer above of 11 sentences reinforce this idea.

508 But is legal language appropriate in healthcare communication? From the point 509 of view of comprehensibility, it seems in conflict with the advice to write in plain 510 English, to avoid jargon and complex language. I am not aware of any studies that 511 have surveyed a patient's responses to the legal information in many documents, 512 though I have presented studies above that show that an increasing majority of 513 patients who have sourced information online choose not to speak with their provider 514 about their findings, which suggests that the encouragement to do so - often expressed 515 in these legal disclaimers - is not successful. We do not know if this is because 516 patients are actively ignoring the legal disclaimers and the encouragement to seek 
517 further information from their doctor or, in fact, because they have not read or 518 understood this part of the document.

On the other hand, legal action against hospitals and doctors is big business: we

520 have already seen that in the US, medical insurance claims reached $\$ 3.7$ billion in

521 2013, but even free-at-the-point-of care medical systems, like the NHS, face

522 increasing claims against it from patients. In just six years, the cost of negligence

523 claims paid out by the NHS Litigation Authority has risen from $£ 0.6$ billion in 2006/7

524 to $£ 18.9$ billion of outstanding liabilities in 2012. (NHS Litigation Authority

525 Factsheet, Factsheet 2, 2012). Many of these litigation cases concern clinical negligence, i.e. errors made during an operation or treatment, though the Citizen's

527 Advice Bureau, a consumer advice agency in the United Kingdom, estimates that 528 communication failure lies behind $20 \%$ of these claims. An example of a recent legal

529 case brought against an NHS hospital by a patient that relates specifically to the 530 provision of information is reported below: Mayday Hospital, accompanied by a friend. He was booked in by a receptionist at 20.26 and was told that it would be up to four or five hours before he would be seen. He was not informed that a triage nurse would examine him within 30 minutes and determine how soon he needed to see a doctor. As he was in pain he decided to go home after just 19 minutes and take paracetamol. Unfortunately, his condition rapidly worsened. He returned to hospital by ambulance and it was discovered that he had an extradural haematoma, but too late to prevent serious brain injury. The experts for the parties agreed that if the claimant had remained in A\&E he would have been treated sufficiently quickly to have avoided the brain damage. The essence of this claim was that the A\&E receptionist owed the claimant a duty of care to give him accurate information about waiting times. The trial judge had accepted Mr D's assertion that had he been told that he would be seen by a nurse within 30 minutes, he would have stayed in hospital and therefore avoided his permanent injury. Lord Justice Jackson gave the main judgment. He said that this case was significant because roughly 100,000 people visit A\&E departments across England every week. He was satisfied that there was no duty upon receptionists to keep patients informed about waiting times. It would not be fair, just or reasonable in his view to impose liability in such circumstances. Were this type of claim to be permitted, litigation about who said what and to whom in A\&E could become prevalent. Trusts might then instruct receptionists to say nothing to patients other than ask for their details, which would be unhelpful. The claimant had been told to wait but chose not to do so. People had to accept responsibility for their actions in his opinion. (NHS Resolution, 2017) 
557 While this extract relates to spoken information, it also has relevance for the role of

558 written information. NHS hospitals, authorities and trusts have to strike the right

559 balance between providing information to patients as a fundamental aspect of patient-

560 centred care, while, at the same time, covering themselves against misinterpretation,

561 misunderstanding or misuse of that information.

562 In the US, legal disclaimers are very visible and appeared in every US

563 information leaflet in the corpus. The disclaimer always appears at the end of the

564 document, as they do in the UK-sourced information, and this fact makes it worth

565 reflecting for a moment on the question of sampling, discussed in 3.1.4. If my patient

566 information documents had been subject to sampling, that is, only a section of each

567 document used - and it is generally the middle - then I would have remained unaware

568 of the legal information that is so evident in much healthcare material. The keywords

569 that led me to this hugely significant area would have been lost.

570 Let us now move from the category Legal to another category of interest, the

571 Medical professional.

573 4.5.2 Medical Professional

574 There are six words for medical professionals in our keyword list, and all 6 revealed

575 by the analysis with the BNC: radiologist, radiologists, physician, physicians,

576 technologist and radiographer. Technologist and radiographer are synonymous, the

577 former is the American term for the latter. Radiologist is the medical specialist who

578 diagnoses and interprets radiographic images. When we look closely at the frequency

579 of these keywords in the corpus, along with the frequency of two other key health

580 professionals working in a radiography department, a nurse and doctor, an interesting

581 picture emerges, as illustrated in Table 12 below. 


\begin{tabular}{lll}
\hline Professional & Raw freq. & $\begin{array}{l}\text { Adjusted freq. per } \\
\text { million }\end{array}$ \\
\hline radiologist & 893 & $1,912.4$ \\
physician & 1388 & $2,927.5$ \\
doctor & 914 & $1,957.3$ \\
radiographer & 181 & 387.7 \\
nurse & 234 & 501.1 \\
\hline
\end{tabular}

We can see that the two jobs that occur with the lowest frequency are nurse

587 and radiographer. Doctor and radiologist have similar adjusted frequencies while

588 physician appears nearly 3 thousand times per million words. This is a word used with

589 great frequency in patient information and is nearly six times as frequent as nurse.

590 Radiologist occurs with nearly 5 times the frequency of radiographer.

These results strongly suggest that there is an underlying focus on the

592 professional who diagnoses and treats: the doctor, the physician, the radiologist. This

593 imbalance is ironic when one considers that a patient attending hospital for

594 radiography may not have any contact at all with the radiologist. The person they will

595 likely have most contact with is the radiographer (aka technologist) and quite possibly

596 a nurse. This privileging of roles in patient information - if that is what it is - seems to

597 mirror the hierarchy seen in medicine, where doctors have more prestige and power

598 than nurses and other allied health professionals.

599 The over-emphasis on 'the doctor' is also seen in healthcare research: Candlin

600 and Candlin (2003), discussed in 2.4.1, refer to the fact that numerous studies of

601 communication by nurses have been carried out by nursing professionals and

602 published in nursing journals but these studies, unlike studies of doctors, are not

603 referenced in applied linguistics or discourse analytic studies (p144). While recently

604 there has been a little more visibility of nursing communication studies in mainstream

605 journals such as the English for Specific Purposes journal (e.g. Lu, 2018; Staples, 
606 2015; Bosher and Stocker, 2015), the number remains small. This finding, along with

607 the large number of keywords from both extractions that relate to treatment and

608 therapy - there are 13 of them - is particularly significant when we consider gender

609 differences in healthcare information-seeking behaviour and linguistic expression of

610 disease and health discussed earlier in this chapter and in chapter 2.

611 Seale and Charteris-Black (2008), as discussed in 2.5.4, found that men in

612 general, and older men in particular, were significantly more likely to refer to

613 specialists, general practitioners and consultants than were women. The professionals

614 named significantly more frequently in my corpus are specialists and general

615 practitioners. Doctor was used by men more or less equally in Seale and Charteris-

616 Black (2008), suggesting that men, but particularly older men, give particular

617 importance to the specialist knowledge of the medical professionals they interact with.

618 Nurse, in contrast, was referred to significantly more often by women; in my data, as

619 we have seen, both nurse and radiographer are referred to far less frequently than the

620 radiologist, physician and doctors.

621 This is not the only evidence that the patient information may contain

622 information that is more relevant to men. Seale et al.'s (2006) finding, discussed in

6232.5 .3 , that men tend to focus on information relating to treatment, to medical staff and

624 to medical procedures is very relevant to my findings in this doctoral study. The

625 semantic categories in my keyword analysis were predominantly those that Seale et al.

626 (2006) highlight as likely to be of more interest to men: Medical staff, Treatment \&

627 therapy, Medical equipment, Radiographic procedures and Examinations and

628 radiographic technology.

629 If women (and men) are (also) looking for information or confirmation in the

630 patient information leaflets of their emotional or mental responses to their looming

631 radiographic examination they will be disappointed: all modifiers of the verb feel in

632 my corpus relate to physical or physiological sensations. e.g. pain, pressure,

633 discomfort, prick, tired, warm, unwell. There were six uses of the adjective anxious,

634 and three of depressed (and two of these from the same document), but no uses of

635 typical expressions of fear or distress that we expect to find in a healthcare 
636 information leaflet relating to a procedure that has such a close relationship with cancer, e.g. worried, scared, frightened, concerned, sad or tearful.

In spite of these well-reported differences, gender is very rarely considered to

639 be a determinant of healthcare, and, as we have seen, rarely appears as such in health

640 policy documents (Gelb et al. 2011). The question is raised, then, of whether gender is

641 considered by healthcare information writers.

642 Healthcare communication writers employed by large charities and those

643 working for the NHS that were contacted during this research for their views on

644 gender and healthcare information either assumed I was referring to transgender

645 (sometimes referred to in their communication as 'third gender') and/or said they did

646 not consider gender at all in the production of their materials:

We (I) certainly don't consider gender when writing materials, in the sense of writing "for" one particular gender over another. I also had a look through our brand guidelines but there is nothing specific about gender when it comes to our [name of charity] tone of voice. We aim to be "inspiring, authentic, confident, frank and human" in all our comms and these values apply across gender boundaries. (S.Newton, personal communication, August 24, 2018)

654 health condition and one which research consistently shows disproportionately affects

655 women. An editorial in the Lancet Diabetes and Endocrinology (Editorial, 2017)

656 states that in Western countries, not only do fewer women than men receive the level

657 of treatment outlined in healthcare guidelines but woman suffering the Type 1 variety

658 have a $40 \%$ higher risk of premature death.

There are similar findings of disparity in healthcare and prognosis for a large

660 number of common conditions (Legato, Johnson \& Manson, 2016) including heart

661 disease (Westerman \& Wenger, 2016), certain cancers (Williams et al., 2017; Yuan et

662 al., 2016), kidney disease (Jindal, Ryan, Sajjid, Murthy, Baines, 2005). Regitz-

663 Zagrosek (2012, p. 596) writes that the scientific literature contains 'more than 10,000

664 articles [that] deal with sex and gender differences in clinical medicine, epidemiology,

665 pathophysiology, clinical manifestations, outcomes and management'. Table 13 below

666 illustrates the sheer volume of papers - which are likely to have to increased since

6672012 - focussing on sex and gender differences. 


\begin{tabular}{|c|c|c|c|c|c|c|c|c|}
\hline Cardiology & $\begin{array}{l}\text { Rheumatology/ } \\
\text { Immunology }\end{array}$ & Pneumology & Nephrology & $\begin{array}{l}\text { Gastro- } \\
\text { enterology/ } \\
\text { Hepatology }\end{array}$ & Neurology & $\begin{array}{l}\text { Endo- } \\
\text { crinology }\end{array}$ & Oncology & Haematology \\
\hline $\begin{array}{l}\text { Hypertension } \\
(414)\end{array}$ & $\begin{array}{l}\text { Lupus } \\
\text { erythematosus } \\
(68)\end{array}$ & $\begin{array}{l}\text { Asthma } \\
(140)\end{array}$ & Renal failure (27) & Hepatitis B (22) & $\begin{array}{l}\text { Multiple } \\
\text { sclerosis } \\
(65)\end{array}$ & $\begin{array}{l}\text { Diabetes } \\
\text { mellitus } \\
(447)\end{array}$ & $\begin{array}{l}\text { Skin } \\
\text { carcinoma } \\
(45)\end{array}$ & $\begin{array}{l}\text { Anaemia } \\
(44)\end{array}$ \\
\hline $\begin{array}{l}\text { Myocardial } \\
\text { infarction (275) }\end{array}$ & $\begin{array}{l}\text { Rheumatoid } \\
\text { arthritis (41) }\end{array}$ & $\begin{array}{l}\text { Lung cancer } \\
\text { (116) }\end{array}$ & $\begin{array}{l}\text { Diabetic } \\
\text { nephropathy (11) }\end{array}$ & Hepatitis C (26) & $\begin{array}{l}\text { Stroke } \\
(129)\end{array}$ & $\begin{array}{l}\text { Obesity } \\
(349)\end{array}$ & $\begin{array}{l}\text { Gastric } \\
\text { cancer (25) }\end{array}$ & $\begin{array}{l}\text { Leukaemia } \\
(49)\end{array}$ \\
\hline $\begin{array}{l}\text { Heart failure } \\
\text { (153) }\end{array}$ & $\begin{array}{l}\text { Systemic } \\
\text { sclerosis (3) }\end{array}$ & $\begin{array}{l}\text { Chronic } \\
\text { obstructive } \\
\text { pulmonary } \\
\text { disease (36) }\end{array}$ & $\begin{array}{l}\text { Glomerulone- } \\
\text {-nephritis (9) }\end{array}$ & $\begin{array}{l}\text { Hepato-cellular } \\
\text { carcinoma (37) }\end{array}$ & $\begin{array}{l}\text { Alzheimer's } \\
\text { disease } \\
(104)\end{array}$ & $\begin{array}{l}\text { Osteo- } \\
\text { porosis } \\
(123)\end{array}$ & $\begin{array}{l}\text { Renal cell } \\
\text { carcinoma } \\
(17)\end{array}$ & $\begin{array}{l}\text { Lymphoma } \\
\text { (34) }\end{array}$ \\
\hline $\begin{array}{l}\text { Atrial fibrillation } \\
\text { (38) }\end{array}$ & $\begin{array}{l}\text { Fibromyalgia } \\
\text { (15) }\end{array}$ & $\begin{array}{l}\text { Pulmonary } \\
\text { hypertension } \\
\text { (12) }\end{array}$ & $\begin{array}{l}\text { Polycystic kidney } \\
\text { disease (12) }\end{array}$ & $\begin{array}{l}\text { Inflammatory } \\
\text { bowel disease } \\
\text { (13) }\end{array}$ & $\begin{array}{l}\text { Epilepsy } \\
(56)\end{array}$ & $\begin{array}{l}\text { Hypo- } \\
\text { thyreoidsm } \\
(33)\end{array}$ & $\begin{array}{l}\text { Bladder } \\
\text { cancer (22) }\end{array}$ & $\begin{array}{l}\text { Thrombo- } \\
\text { cytopoenia } \\
\text { (6) }\end{array}$ \\
\hline $\begin{array}{l}\text { Coronary heart } \\
\text { disease (207) }\end{array}$ & $\begin{array}{l}\text { Sjögren's } \\
\text { syndrome }\end{array}$ & $\begin{array}{l}\text { Pulmonary } \\
\text { embolism } \\
(110)\end{array}$ & $\begin{array}{l}\text { Renal artery } \\
\text { stenosis }(0)\end{array}$ & $\begin{array}{l}\text { Colorectoral } \\
\text { cancer (24) }\end{array}$ & $\begin{array}{l}\text { Parkinson's } \\
\text { disease } \\
(69)\end{array}$ & $\begin{array}{l}\text { Hyper- } \\
\text { thyreoidsm } \\
\text { (16) }\end{array}$ & $\begin{array}{l}\text { Thyroid } \\
\text { carcinoma } \\
(16)\end{array}$ & Purpura (2) \\
\hline $\begin{array}{l}\text { Cardiomyopathy } \\
\text { (41) }\end{array}$ & $\begin{array}{l}\text { Ankylosing } \\
\text { sponylitis (11) }\end{array}$ & $\begin{array}{l}\text { Sarcoidosis } \\
\text { (6) }\end{array}$ & $\begin{array}{l}\text { IgA Nephropathy } \\
\text { (2) }\end{array}$ & $\begin{array}{l}\text { Autoimmune } \\
\text { Hepatitis (2) }\end{array}$ & $\begin{array}{l}\text { Muscular } \\
\text { dystrophy } \\
\text { (11) }\end{array}$ & $\begin{array}{l}\text { Morbus } \\
\text { Addison/ } \\
\text { Cushing } \\
\text { disease (5) }\end{array}$ & $\begin{array}{l}\text { Pancreatic } \\
\text { carcinoma } \\
(10)\end{array}$ & $\begin{array}{l}\text { Agranulo- } \\
\text { cytosis (0) }\end{array}$ \\
\hline
\end{tabular}

671 It is misguided, then, to believe that being human trumps sex and gender

672 differences in health when the evidence from the scientific literature is clear. This

673 evidence suggests strongly that biological sex and gender have a relationship with the

674 manifestations of, experiences of and outcomes of disease. This, in turn, suggests that

675 a one-size-all approach to healthcare information is not only inappropriate but may

676 also result in information that is unwittingly gender- and age-biased: my examination

677 of the keywords in patient information for radiography strongly suggests that the

678 content prioritises information that is likely to be of particular interest to older men.

679 Without acknowledgement of these differences, and without data that can inform

680 public health campaigns and patient materials, there is a real danger of health

681 messages not being transmitted.

682 The increasing numbers of people who use internet forums for healthcare

683 advice may, in part, be explained by the need to find information other than that which

684 is published or presented officially. In a study of internet forums relating to chronic

685 cough (Sinha, Porter \& Wilson, 2018), traditional medical advice was sought and

686 given (and judged to be of good quality by raters), along with emotional support for 
the psychological stress associated with the condition. Of note is the attitude towards medical consultations:

714 it is interesting to compare the comparative clarity of the description with that found

715 in an US leaflet, which is more technical and lexically complex:

Chronic cough is a condition with which patients often visit their doctor multiple times. Our data show forum users avoiding doctors' appointments after bad experiences or lack of effective treatments, citing them as a waste of time. These patients are lost to follow-up in the medical system, but may frequent online health forums, seeking advice from other sources. (Sinha et al., 2018, n.p)

We have already seen in Chapter 2 that the 'biomedical discourse' that Dixon (2002) refers to is a feature of much pharmaceutical patient information. My keyword extraction reported in this chapter strongly suggests that it is also the primary discourse in procedural patient information. 'Bio-technical' is also an appropriate term. The appropriacy of this discourse for all patients, and whether it provides the information all patients would like to have is an area worth further investigation.

Let us now turn to another category that Seale et al. (2006) found to be particularly interesting to male healthcare-information seekers, and one that falls under the heading of bio-medical discourse: Treatment and therapy.

\subsubsection{Treatment/therapy}

This was a large category of keywords with 6 words, predominantly nouns, in each list. There were no words in common, however. The generic term medications appears in the extraction with the BNC and thinners, a reference to blood thinners in the extraction using the Radiography corpus, while two descriptive adjectives for the type of medical procedure also appear: interventional and non-invasive. The rest of the words were specific therapies: chemoembolization, brachytherapy, embolization, cryotherapy, kyphoplasty, vertebroplasty, thrombolysis and ablation.

In many cases, these words appeared only in US materials and were described in fairly complex terms. There is one example of cryotherapy in the UK materials and 
(12) Cryotherapy is an alternative technique that freezes tissues instead of burning them. It involves insertion of small needles (cryoprobes) through the skin, which circulate very cold gas and freeze the tumour by producing ice. (UK)

(13) During cryotherapy, liquid nitrogen or argon gas flows into a needle-like applicator (a cryoprobe) creating intense cold that is placed in contact to diseased tissue. (US)

As I have said, the vast majority of the named therapies in the category were

\section{3 found in US materials. While it seems possible that UK patient information is}

724 avoiding obvious mention of something considered frightening and unpleasant, there

725 is another, more likely, explanation: the majority of the 94 documents sourced from

726 the NHS and the Royal College of Radiologists was information pertaining to

727 diagnostic radiography or procedures involving radiography, such as angiography.

728 Diagnostic radiography is that which is used to diagnose or rule out a disease. On the

729 other hand, the 136 documents sourced from RadiologyInfo.org, also contained some

730 information about therapeutic radiography, which is radiography used for the

731 treatment of cancer. This partly explains the quantity of precise therapies listed.

732 Closer inspection of the corpus also revealed that a very small number of long, 733 therapeutic documents were responsible for the appearance in the keyword list of a

734 number of the keywords: chemoembolization (3 documents; 138 per million words);

735 embolization (5 documents; 414 per million words); kyphoplasty (1 document; 62 per

736 million words). The name of the specific therapy was repeated frequently in each

737 document, though it is clear that these words are used quite idiosyncratically and only

738 one word, embolization, is present in 5 documents. Kyphoplasty appears 29 times but

739 only in one document. This finding underlines Scott's (2010) reminder that statistical

740 chance (and not necessarily linguistic value) is a factor in keyword extraction (p50),

741 and that keywords are useful for suggesting areas worth investigation, but they are not

742 always, in themselves, of much value.

743 4.5.4 Information

744 Leaflet, web (site) and download were categorised under Communication, a heading 745 that covers information from print and non-print sources, such as websites. Download 
746 and web(site) appeared together on RadiologyInfo.org's website and were used to tell

747 patients to return to the site to check for, and download, updated or further

748 information. Leaflet was also sometimes used to encourage patients to read more

749 information:

750 (14) If you would like more information about this, please ask a member of staff for

$751 \quad$ a leaflet called What to do if the contrast injection leaks out (extravasation)

752 With this as determiner, leaflet was also used to focus attention on the purpose of the 753 information being read:

754 (15) This leaflet will give you some general information about the clinic. If you

755 have any further questions, please speak to a doctor or nurse caring for you

756 (16) This leaflet contains information on gadolinium (also known by its brand 757 name Dotarem $\left.{ }^{\circledR}\right)$, which is a contrast (dye) used during MRI scans

758 There were also some examples of a hospital leaflet referencing their no-

759 smoking policy; in these cases, the patient is instructed to read the leaflet with a polite

760 imperative. In this directive, our is always used in place of the or this, perhaps to

761 emphasise that this is a policy 'owned' by the entire institution or hospital (and thus to 762 be taken seriously).

763 (17) Please read our leaflet 'Policy on Smoke Free NHS Premises' to find out more

764 As we have seen in 4.5.1, this leaflet is also used as part of a disclaimer where 765 the idea of its scope being limited is expressed:

766 (18) Legal notice. Please remember that this leaflet is intended as general 767 information only. It is not definitive, and the RCR and the BSIR cannot accept $768 \quad$ any legal liability arising from its use

769 (19) Some of your questions should have been answered by this leaflet, but $770 \quad$ remember that this is only a starting point for discussion about your treatment $771 \quad$ with the doctors looking after you. 
772 The message to patients seems to be: read our leaflets; they should answer most

773 of your questions. If they do not answer your questions, read some more leaflets or

774 look at another website. However, any information you read must be considered

775 general information, not always specific to you and perhaps not even relevant to you.

776 Communication with medical professionals, particularly the patient's doctor, is

777 frequently encouraged and it is likely that this encouragement is motivated by legal

778 concerns. Legal is the biggest category in the analysis, as we saw in 4.5.1, while

779 disclaimers and legal statements in general are very common in the patient

780 information collected for the corpus, even when presented in an indirect way. Subtle

781 disclaimers such as the above are far more common in the UK-patient information,

782 while more formulaic, legalese is used in the US-information.

$783 \quad 4.5 .5$ General

784 There were a number of the words classed as General: please, resume, outweighs,

785 loose-fitting, piercings and jewelry. The latter 3 are found in the instructions that

786 patients are routinely given in preparation for an exam, while resume refers to the

787 post-exam recovery period:

788 (20) You should wear comfortable, loose-fitting clothing for your ultrasound exam

789 (21) We will ask you to remove all jewellery and body piercings before the scan as

790 the scanner uses a very strong magnet

791 (22) Jewelry and other accessories should be left at home if possible

792 (22) You will be able to resume all other normal activities 8 to 12 hours after the 793 exam

$794 \quad$ Please also appears in this category and is used very frequently in the corpus:

795759 occurrences which corresponds to a $1,625.45$ per million words. What is

796 particularly interesting is that please is used exclusively with a verb in the imperative,

797 e.g. please ask your doctor for more information. Some of these imperative structures

798 functioned as invitations and offers, particularly of further information or advice, 
799 (23) If you have any questions or concerns, please do not hesitate to speak to a

800 doctor or nurse caring for you

801 though many of the structures are imperatives and are obliging the patient to do

802 something:

803 (24) Arriving at the clinic: Please report to the receptionist on arrival.,

804 (25) Please make sure that you understand the risks and benefits of the procedure

$805 \quad$ and that it has been explained to you in the detail you need.

806 (26) If you are not able to attend, please let the department know in good time

807 (27) You will be asked to undress in a cubicle and you will be given a cotton gown to $808 \quad$ wear; please bring your own dressing gown.

809 Many of the uses of please referenced further communication between the patient and

810 the hospital or care provider, in common with leaflet and web as we saw earlier in

811 section 4.5.4,

812 (28) If you have a query, please ring your breast surgeon's secretary or a breast $813 \quad$ care nurse

814 (29) If you have any questions about the procedure please ask the doctor who has 815 referred you for the test or the department which is going to perform it.

816 Other uses were as part of a legal disclaimer, a category discussed earlier in this

817 chapter in section 4.5.1,

818 (30) All information is provided "as is" without express or implied warranty. Please

819 visit the RadiologyInfo Web site at http://www.radiologyinfo.org to view or 820 download the latest information.

821 (31) Please remember that this leaflet is intended as general information only. It is 822 not definitive, and the RCR and the BSIR cannot accept any legal liability $823 \quad$ arising from its use. 
824 There were just seven uses (50 per million words) of please + not + verb; four of these

825 examples invited patients to seek more information, while three of these also

826 functioned as negative obligations,

827 (32). If you have any questions or concerns, please do not hesitate to speak to a 828 doctor or nurse caring for you.

829 (33) Please do not bring children with you to the department. This is to avoid

$830 \quad$ exposing them to unnecessary radiation.

831 In the corpus of patient information in this doctoral study, please was used with 832 imperatives to give instructions but also to invite and encourage an exchange of

833 communication. Table 14 below shows the 10 most frequent verbs collocated with

834 please in order of descending collocational strength.

835

836

837

838

839

840

841

842

843

844

845

846

847 
Verb Example of use in the corpus

1. visit for more information...please visit the KIC on the Ground floor

2. contact if you have any questions or concerns about ablation, please contact...

3. ask please ask your doctor for more information

4. bring please bring an overnight bag with you to hospital

5. remember please remember that this information is intended as general information only

6. tell please tell us before the injection if you think you might be pregnant

7. let please let us know if you are taking any antiplatelet medicines

8. telephone please telephone xxxx to cancel or make changes to your appointment

9. consult $\quad$ please consult with your physician as to whether or not you will be admitted

10. read please read out leaflet: Policy on smoke-free premises

849 Please collocates most strongly with visit, contact and ask. All three of these

850 verbs are inviting and encouraging communication between the patient and the

851 professional, which underlines how important an aspect this is in procedural patient

852 information. The remaining collocates - the majority - are all instructing the patient to

853 do something, suggesting that this is also an important function of patient procedural

854 materials. I will pick this topic up again in Chapter 6, where I report the results of my

855 analysis of modal verbs of obligation.

856 Before I conclude this chapter, I would like to refer not to a category, but a

857 significant defining feature of patient information that has been clearly illuminated by

858 the two keyword extractions, that of the use of patient-friendly vocabulary alongside

859 complex medical vocabulary. 
861 The complexity of vocabulary in radiography, and in medicine in general, has been

862 the focus of many studies, as we saw in Chapter 1. Studies repeatedly show that

863 patients struggle to understand medical consultations (Chapman et al., 2014;

864 O'Connell et al., 2014) and readability, as we have seen, is a constant concern. (e.g.

865 Morony et al., 2015).

866 In my two keyword extractions, a total of 100 keywords were examined. Three

867 categories are shown below in Table 15 which illustrate the kinds of words that are

868 being used as patient-friendly terms, and some of their medical equivalents.

869 Table 15 Patient friendly keywords with their medical equivalents

$\mathrm{BNC}$ as ref. corpus $\quad$ Category $\quad$ Radiography as ref. corpus

CT, x-ray, x-rays, ultrasound, MRI, tomography,

anesthesia, sedation, anesthetic, intravenous

\section{Radiographic \\ modality}

Inaudible, sonar, loaf, magnets, box-like, television-like numb, sting, prick

871 television-like and loaf which are all used to describe or explain aspects of a scanning

872 machine to the patient. While magnet may be comprehensible if you know that MRI

873 stands for magnetic resonance imaging, box-like and television-like are less so;

874 without seeing the data, loaf seems entirely out of place in the context of radiography.

875 Examining the words in context reveals how these words are used:

876 (34) Some of the magnets used for MRIs are like narrow tunnels and others are more open.

878 (35) The CT scanner is typically a large, box-like machine with a hole, or short 879 tunnel, in the center. 
907 (43). Infants and young children usually require sedation or anesthesia to complete 908

(36) The equipment typically used for this examination consists of a radiographic table, one or two x-ray tubes and a television-like monitor that is located in the examining room.

(37) CT imaging is sometimes compared to looking into a loaf of bread by cutting the loaf into thin slices

We also have inaudible and sonar which are both used to refer to the ultrasound examination.

(38) The transducer sends out inaudible high-frequency sound waves into the body and then listens for the returning echoes from the tissues in the body. The principles are similar to sonar used by boats and submarines

Examples (36) and (37) are good examples of simplified medical language being anything but (37) is simply obscure in its imagery. When does cutting a loaf involve looking into it? (38) assumes vocabulary knowledge with inaudible and cultural knowledge with sonar that just cannot be assumed.

I would like to conclude this section by considering three further words that showed up our keyword list, numb, sting, prick. They appear in the category Medical: other and were listed when using the Radiography corpus as a reference corpus. They also very neatly appear alongside the medical procedures they are referencing (as do the keywords 32-36) which were listed as keywords with the BNC as reference corpus: anesthesia, sedation, anesthetic, intravenous. How these items are used in the corpus is illustrated below in $37-41$.

(39) ... a local anaesthetic will be injected into your groin area. This will sting at first but will then numb the area so that you do not feel any pain.

(40) Your physician will numb the area with a local anesthetic

(41) It may sting a little when the local anaesthetic is injected.

(42) You will feel a slight pin prick when the needle is inserted into your vein for the intravenous line (IV) an MRI exam without moving 
910 and would, I feel, frighten many parents.

911 There is also a vocabulary comprehension issue in this sentence that Clerehan et

912 al. (2005) raise, discussed in 2.6.1, which is that of presenting two terms as synonyms

913 in the same sentences, separated by the word 'or', e.g. 'Methotrexate may cause a

914 reduction in the number of white cells or platelets in the blood' can be doubly-

915 confusing for patients if they are unfamiliar with either or both terms; it is also the

916 case that sometimes the second word is a synonym but sometimes a new, additional

917 word. How is the patient expected to know this? There are 39 examples of sedation

918 used with anesthesia in this way in my corpus. Is sedation the same as anesthesia?

919 Out of a random sample of 10 sentences of content word + or + content word

920 from the patient information corpus, I found 4 that could be taken to be synonymous.

921 E.g. sometimes a small plug or stitch is placed in the artery; other risks or

922 complications include... It is important to remember, too, that health literacy is not

923 literacy. Zarcadoolas (2011), discussed in 2.6.2, refers to the variety of knowledge that

924 we bring to the interpretation of a text: the social, cultural and environmental aspects

925 of health literacy. We have to know already something about sedation and anaesthesia

926 to be able to know whether these terms are synonyms; we need to know something

927 about medical vocabulary to feel confident that risks means more or less the same

928 thing as complications.

929 There are 6,685 (14,316.34 per million) word + or + word combinations in my

930 corpus. Not only is this structure very frequent in healthcare materials, but we have

931 seen that the two content words (often nouns) are only sometimes synonymous.

932 Generally, no information is given in the text to help readers with this interpretation.

933 The complexity of health literacy means that a lot of knowledge -and some of it is

934 specialist, medical knowledge - is required by readers to be confident that they fully

935 understand these sentences. The factors reported here strongly suggest that this is an

936 aspect of healthcare materials that warrants further research. 
938 My keyword analysis carried out with two different reference corpora uncovered a

939 number of areas of linguistic interest.

940 An overarching theme in the corpus is the role of patient information in

941 healthcare education, and, more particularly, the limits to that role. These limits are

942 expressed in clear, legal disclaimers in the US-sourced information, while the UK

943 materials prefer a linguistically softer approach that encourages the patient to not rely

944 on anything they have read (as it may not be accurate or appropriate) and to speak

945 about the information with their healthcare provider. As we have seen, however,

946 considerably more than half of people who engage in ehealth information-seeking do

947 not subsequently refer to their doctor to discuss it. We do not know the reasons for

948 this though they seem to be various and complex. It is also the case that some legal

949 disclaimers are long and linguistically complex, which may be very off-putting for

950 readers (who may not read them at all).

951 On the other hand, disclaimers that try to be non-threatening and non-

952 disclaimer-like may not be understood to even be disclaimers. Many people report

953 trusting hospital-produced information and to rate it as highly as the information their

954 doctor provides. That patients trust the printed information seems at odds with the

955 message that information often contains (in the form of non-threatening disclaimer):

956 that it is irrelevant or not up-to-date or appropriate, which is the message that much

957 UK materials seem to transmit. It would be interesting to find out what the impact is

958 of messages such as this in healthcare materials.

959 Another important theme that emerged was the reflection of the power

960 hierarchy in the naming of professionals. Medical doctors and specialists

961 (radiologists) were referred to far more often than the radiographers and technologists

962 - the very personnel who are responsible for performing the radiographic

963 examinations and therapeutic sessions - and considerably more often than nurses, who

964 are often present for radiographic procedures. One explanation is the focus on the

965 significance of diagnosis for the patient reading the material. As we have seen, most,

966 if not all, radiographic examinations can be (and very often are) used for finding or 
967 excluding cancer and a diagnosis is not given by a radiographer or a nurse. While this

968 may be the case, (and many appointments for radiographic exams do not relate to

969 cancer), not referring by name to the healthcare professional performing the

970 examination is a strange omission. Many patients will have no contact with a

971 radiologist: the results of a radiographic examination will be sent to the referring

972 doctor or physician.

973 The keyword analysis reported in this study also raised some very interesting

974 questions about the kind of information being presented in procedural patient

975 information. It is, in common with much pharmaceutical patient information, a

976 discourse that is overwhelmingly biomedical and biotechnical. This may be the result

977 of the technological nature of this branch of medicine, though it may be, also, that

978 healthcare information materials habitually present the kind of information that only a

979 small section of the population finds satisfying: older men. All age groups and both

980 sexes undergo radiographic examinations, and it is possible that other kinds of

981 information is wanted, information that is currently not present in the types of

982 published materials that make up my corpus.

983 Another area of interest revealed by the keyword extraction relates to how

984 information is presented in what we can refer to as 'or' structures. There is an

985 assumption on the part of many materials writers that readers have the capacity to

986 judge whether the content words in these structures is additional or synonymous.

987 Given that many of the words relate to medicine and radiography parsing the text

988 demands a high level of health literacy. Understanding a word when it is used alone,

989 in a clear context, may cause less problems than trying to decide whether two words,

990 both of which you think you know, are synonymous. We return to the notion of

991 understanding, raised by John Skelton in Chapter 1. The issue is not really whether we

992 know what words mean, but how we understand them. Presenting two items in a

993 sentence that are often very similar, but leaving it up to the reader to decide just how

994 similar, seems an unnecessary complication in healthcare materials, which, after all,

995 are striving for clarity. 
996 We stay with questions of structure in the next chapter, in which I present the

997 results of my analysis of 4-word lexical bundles in patient information for

998 radiography.

999

1000

1001

1002

1003

1004

1005

1006

1007

1008

1009

1010

1011

1012

1013

1014

1015 
1017 This chapter complements the keyword analysis described in the preceding chapter by

1018 reporting on an analysis of the frequency, distribution and discourse function of four-

1019 word lexical bundles in patient information for radiography. Both keywords and an

1020 analysis of lexical bundles can reveal aspects of the lexical characteristics and lexical

1021 patterns found in patient information.

1022 Lexical bundles are multi-word lexical sequences that frequently reoccur in a

1023 register, e.g. in the light of and at the end of. They have been described as

1024 'characteristic features of language use in particular settings' (Hyland, 2008, p8) and

1025 as 'text building blocks' (Biber et al., 2004, p443). Usually transparent in meaning,

1026 they tend to be structurally incomplete and often bridge two structural units, i.e. a

1027 clause or phrase, very often functioning as the pragmatic head of an utterance and

1028 acting as an interpretative frame for the discourse that follows (Biber and Barbieri,

1029 2007, p. 8). Lexical bundles are generally made up of grammatical words while the

1030 keywords discussed in the previous chapter tend to be content words, belonging to the

1031 noun, verb and adjective class predominantly

1032 Variously referred to in the literature as formulaic sequences (Wray 2002;

1033 Schmitt and Carter 2004), lexical bundles (Biber \& Conrad, 1999), n-grams (Stubbs

1034 and Barth 2003) or lexical phrases (Nattinger \& DeCarrico, 1988), lexical bundles

1035 have received a fair amount of attention in the literature, though not, as we shall see,

1036 in the healthcare discourse literature. While different terms are used for these

1037 multiword sequences, for McEnery and Hardie (2012, p110), lexical bundles are,

1038 'methodologically and technically', simply recurring sequences of $n$ words, i.e. n-

1039 grams. They add that the term 'lexical bundle' has become associated with the work

1040 of Biber and colleagues on register description, and on their focus on the structural

1041 and functional interpretation of lexical bundles. As it is the structural and functional

1042 interpretation that interests me, it is Biber and colleagues' terminology and approach

1043 that I have chosen to use. While earlier studies on multiword units relied on intuitive

1044 lists of prefabricated expressions (e.g Pawley and Syder, 1983; Nattinger and

1045 DeCarrico, 1992), corpus software has permitted an evidence-based approach to 
1046 studies, with Altenberg's study (1998) of the phraseology of spoken English, being 1047 one of the earliest.

1048 In this chapter, after an overview of the methodological steps taken to extract 1049 lexical bundles, which has been presented in full in chapter 3, I will present the results

1050 of my analysis of the bundles, describing their frequency and their distribution in 1051 patient information for radiography. As I have already reported in chapter 3, for this 1052 doctoral study the analysis was restricted to 4-word lexical bundles. These bundles are

1053 less common than 3-word bundles, which occur very frequently in both spoken and 1054 written discourse (Conrad and Biber, 2004) but are not as rare as 5-and 6-word 1055 bundles, meaning an analysis of 4-word bundles results in a sufficient, but not an 1056 overwhelming quantity, of data. This will be followed by an analysis of the discourse

1057 functions and the communicative purpose of the identified bundles in patient 1058 information.

1059 I begin, however, with an overview of the literature of the lexical bundle 1060 literature. As we have seen in chapter 2, unlike a keyword analysis, a lexical bundle 1061 analysis has rarely been used in healthcare discourse studies, though in studies of 1062 academic registers, however, lexical bundles have been the focus of many studies.

\section{$1063 \quad 5.1$ Lexical bundles in the literature}

1064 Lexical bundles are a powerful tool for the understanding of the unique characteristics 1065 of registers (Biber 1988) and have been described as the 'building blocks of discourse' 1066 (Biber, Conrad \& Cortes 2004, p. 401). Bundles are found in both spoken and written 1067 discourse though their frequency and distribution differ. Conrad and Biber (2005) 1068 showed that the 3-word bundle I don't know appears repeatedly in conversation at 1069 over 1,000 times per million words, and, while individual bundles also appear often in 1070 academic prose, the most-used items appear far less frequently, at between 200 and 1071400 times per million words (Conrad and Biber, 2005). Conversation, then, might be 1072 said to possess a repetitive quality that is generally not seen in written discourse.

1073 The range and frequency of lexical bundles, however, are not solely defined by 1074 the mode of discourse. A seminal study by Biber et al. (2004) found that university 1075 classroom talk uses a wider range of types and higher frequency of lexical bundles 
than informal conversation and academic prose, evidence, says Barbieri (2018) of the

1077 communicative purposes of classroom teaching, 'which combines the informational

1078 focus typical of academic prose with the expression of personal stance and

1079 interpersonal meanings typical of casual conversation.' (p. 253)

The university is the focus of many studies in the literature, and while some

1081 studies have focussed on or included spoken university registers in their studies (e.g.

1082 Biber et al., 2004; Biber \& Barbieri, 2007, Csomay, 2013), the literature on academic

1083 writing predominates, almost certainly because of the increasing importance of

1084 English in global academia and because of the rising number of foreign students

1085 studying in English. The research article has been a particular focus, with studies that

1086 identify bundles specific to different sections of the research article, and explore the

1087 functions of those bundles (Cortes, 2013; Jalali et al., 2015).

The frequency and type of bundle have been found to vary considerably across

1089 different disciplines (Cortes, 2002; 2004; Durrant, 2017; Hyland 2012; 2008a; 2008b).

1090 Durrant (2017), used Hyland's (2008a) taxonomy, findingbb evidence for a clear

1091 distinction between the hard and soft sciences, with two further groupings of life

1092 sciences and commerce sitting between the two. In addition, evidence is found of

1093 disciplines which are essentially heterogeneous in nature such as engineering and

1094 cross-disciplines which draw on a variety of influences, such as the health sciences.

Difference in frequency and type of bundle are found between and within 1096 spoken and written academic modes as we have already seen (Biber et al., 2004; Biber 1097 and Barbieri, 2007), while variation has also been seen between expert writers and 1098 novice writers (Cortes, 2004), both in the range, type and function of the lexical 1099 bundles that they use. Cortes (2004) found that university students of history and 1100 biology rarely used lexical bundles in their writing, and when they did, their use did 1101 not correspond to the uses of bundles employed by professional authors.

More recently, studies have increasingly focussed on language background,

1103 finding that L1 and L2 speakers of English use different kinds and quantities of lexical 1104 bundles (Ädel and Erman, 2012; Bychkovska and Lee, 2017; Chen and Baker, 2010;

1105 Pan et al, 2016). It is perhaps less surprising that there are differences between L1 and 1106 L2 users, but it turns out that the differences are not related to frequency alone, but 
1107 also to the function, the structure, and type of the bundles. Pan et al. (2016) compared

1108 the use of bundles in telecommunications journal articles written by English L1

1109 academics with those of Chinese peers writing in English. The study found that while

1110 both groups used lexical bundles, the L2 professionals preferred bundles made up of

1111 verbs plus clause fragments, in particular, passive structures, while L1 speakers used

1112 more bundles made up of noun plus prepositional phrases. Similar results were found

1113 by Efandiari and Barbary (2017) in their comparative study of English and Persian

1114 writers of psychology research articles.

1115 Staples et al. (2013) looked in more detail at the development of bundles in L2

1116 writers, focusing on their frequency, function, and degree of fixedness. For a corpus,

1117 the study used essays written by candidates in the TOEFL iBT exam. With important

1118 implications for language teaching, there were few differences in the fixed versus

1119 variable slot bundles used by different proficiency levels of learners, and while lower

1120 levels actually used more bundles, closer inspection revealed that many of these

1121 bundles were copied from the essay prompts. This last finding echoes that of Wray

1122 and Perkins (2000) who found that L2 learners are much more likely to rely on the

1123 imitation and repetition of formulaic sequences (p10). Referential bundles, e.g that

1124 kind of thing; the end of the; as shown in fig, were very rarely used by any candidate,

1125 irrespective of proficiency level. Pan et al.'s study also found that noun plus

1126 prepositional phrase bundles (which many referential bundles tend to be) were not

1127 used by their learners, irrespective of level.

1128 Lexical bundles, then, need to be learned. The evidence presented above (e.g.

1129 Cortes, 2004) that expert and novice L1 writers use different quantities and types of

1130 bundles and the L2 users, in addition to using different types of bundles, also use

1131 different structural types of bundle (e.g. Pan et al. 2006) suggests that bundles are not

1132 something that are easily acquired and may need to be overtly presented and taught by

1133 ESP teachers. Nesselhauf (2005, p. 69) describes L2 learners as using bundles like

1134 'lexical teddy bears', a reference to students' tendency to overuse a small range of

1135 (favourite) bundles. Additionally, referential bundles, which are a feature of expert

1136 and L1 writing and very prominent in informational text (I use text to refer to both

1137 written and spoken language), are generally avoided by L2 learners of English

1138 (Staples et al., 2013). It is certainly the importance of bundles, combined with their 
1139 proliferation and the need to learn them in order to become a member of the particular

1140 discourse community - a need that is the same irrespective of language background -

1141 that has resulted in the majority of lexical bundles studies focussing on the academic.

1142 What of studies of non-academic registers, or studies that have compared

1143 bundles across registers from the same domain? A small number of domain-specific

1144 studies have looked at lexical bundle frequency and function in a range of registers

1145 within one domain, e.g. law (Breeze, 2013; Gozdz-Roszkowski, 2011) and pharmacy

1146 (Grabowski 2013; 2015) - Grabowski (2015) has been presented in chapter 2 - while

1147 studies of non-academic registers include Barbieri (2018), who looks at lexical

1148 bundles in blogs. Barbieri (2018) finds that blogs are characterised by a combination

1149 of stance expressions and make heavy use of verb-phrase structures. These types of

1150 bundles, as we have seen earlier in this chapter, are more commonly found in

1151 conversation (Conrad \& Biber, 2005). What is particularly interesting, however, is

1152 that blogs also rely on referential bundles and narrative expressions, a bundle

1153 combination reflects both the communicative purpose of a blog and the mode.

1154 Lexical bundle studies in medical registers are generally limited to medical

1155 research papers (Jalali \& Moini, 2004; Jalali et al., 2015; Mazzi, 2016), with

1156 occasional exceptions: Kopaczyk (2013) looked at 3-word lexical bundles in Early

1157 Modern English medical writing, and there have been no studies of lexical bundles, to

1158 my knowledge, in other medical registers. One reason for the absence of non-

1159 academic focussed studies may well be the emphasis in the literature on the academic,

1160 as I have previously discussed. This emphasis might explain why the language of

1161 medicine, in these studies, is treated as homogenous, instead of a genre that is made

1162 up of a wide variety of clinical specialities. Medical students, after all, do not

1163 specialise while they are still students. Studies of research papers or abstracts tend to

1164 use a corpus made up of articles from a range of specialities. Mazzi (2016) uses

1165 articles from journals from 14 different specialities, selected after asking for advice

1166 from his university medical and scientific colleagues. The journals are the British

1167 Journal of Dermatology, Journal of the American Academy of Dermatology, Blood

1168 Cells, Molecules and Diseases, Proceedings of the National Academy of Sciences of

1169 the United States, Cancer Research, British Journal of Haematology, Artificial

1170 Organs, Proteome Science, Clinical Chemistry, Journal of Pharmaceutical and 
1171 Biomedical Analysis, Science and Current Opinions in Genetics and Development.

1172 My understanding of these specialities is that they might be expected to demonstrate

1173 not only different vocabulary but, as Durrant (2017) shows, a different use and range

1174 of lexical bundles. Mazzi (2016) cites Hunston (2008) in suggesting that the well-

1175 established meanings and functions of 'phraseologies' (p. 14) override any subject

1176 differences, though if the objective of such lexical bundle studies is to help train

1177 students to write 'as a medical researcher [which] implies being capable of talking to

1178 the expert members of the relevant discourse community in ways they find most

1179 effective', I believe subject-specific studies would be more useful.

1180 Aside from medical research and historical investigations, other medical

1181 registers have yet to be studied. Given the success of a lexical bundles analysis in

1182 revealing the true communicative purpose of a text, as reported by the studies I have

1183 referred to in this section, and the importance and ubiquity of written patient

1184 information, a bundle analysis of these healthcare materials seems overdue.

\section{$1185 \quad 5.2$ The discourse function of lexical bundles}

1186 Lexical bundles serve important discourse functions and can be broadly categorised as

1187 referential (e.g. at the same time; the rest of the), discourse organising (let's have a

1188 look; if you have any) and stance conveying (it's not possible to; if you want to) (Biber

1189 et al., 2004a; Conrad \& Biber, 2005; Cortes, 2004; 2006; 2013). These categories

1190 contain further, more defined sub-categories, e.g. the category Discourse-organising

1191 bundles contains two sub-categories of bundle: Topic introduction and Topic

1192 elaboration and clarification. The taxonomy is presented in full 3.5.2.7 and is

1193 summarised below in Figure 8 


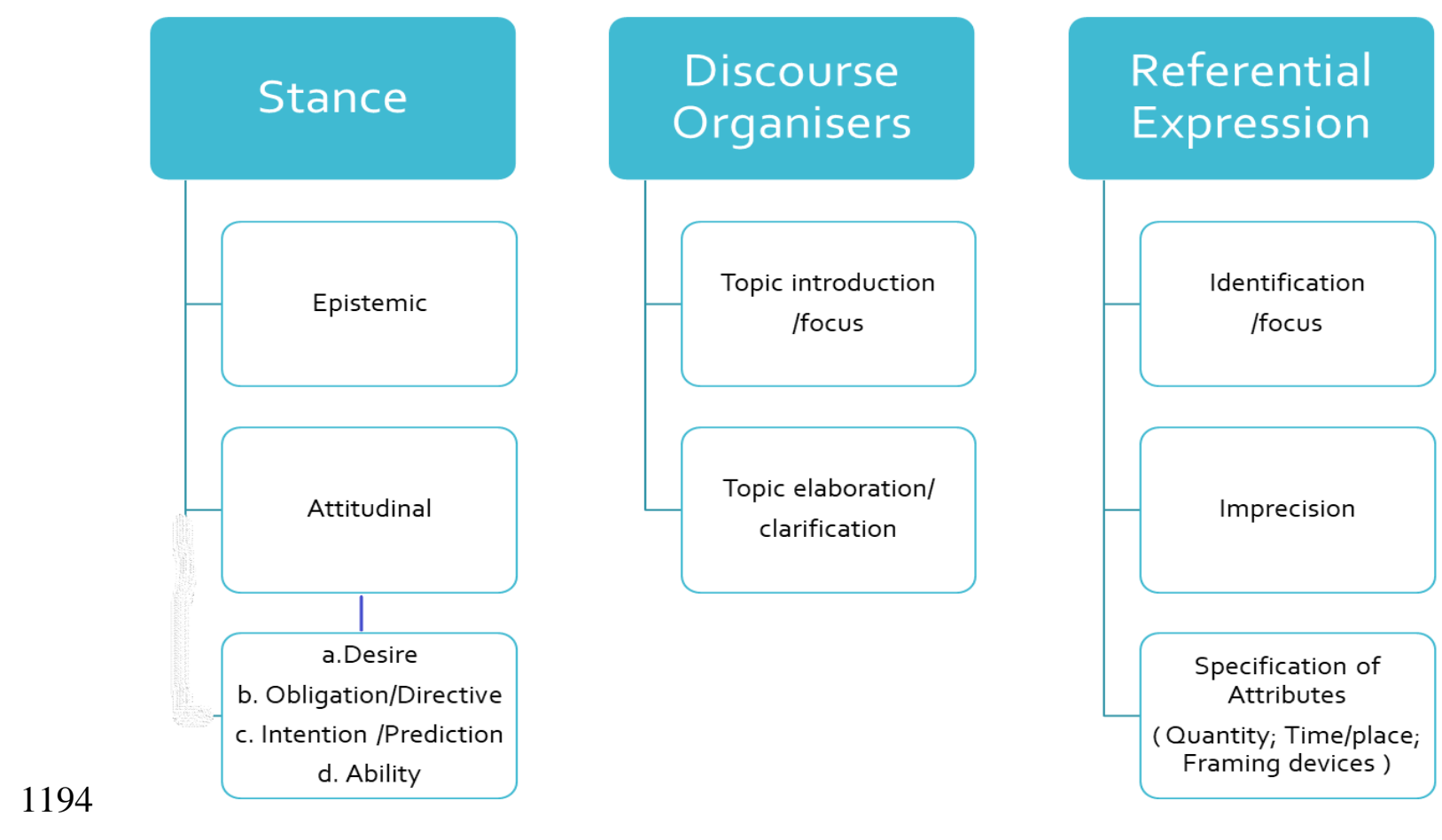

1195 Figure 7 Taxonomy of discourse categories, after Biber et al., 2004a; Conrad and Biber, 2005.

1196 Assigning a discourse function to the bundle is a significant step in the methodology,

1197 which is re-visited below and presented in detail in chapter 3.

$1198 \quad 5.3$ Methodology

1199 As this is one study in a larger investigation of patient information, the focus of the

1200 analysis was solely on 4-word bundles in order to avoid unmanageable quantities of

1201 data. Sketch Engine refers to 'n-grams' and thus a search was carried out limiting the 1202 span of the n-gram to 4 words.

1203 The cut-off point was set at 20 per million words. This means that all 4-word 1204 bundles appearing at least 20 times in the corpus were included. While 40 is also a 1205 common cut-off in similar studies (e.g Biber and Barbieri, 2007; Goźdź-Roszkowski, 1206 2011), the cut-off point it is always fairly arbitrary and much depends on the size of 1207 the corpus, the researcher's preferences and the length of the bundle. A lower cut-off 1208 point, for example, is generally selected for the rarer, 5-and 6-word bundles (e.g 1209 Cortes, 2013). The small size of the corpus used in this thesis, just over 400,000 1210 words, and my impressions of the quality of the data between 20 and 40 per million 1211 words (pmw), were factors in the decision to use the lower cut-off. There were a 
1212 number of bundles that I felt were interesting to investigate further between the 20 and

121340 per million cut-off point that would have been excluded by choosing the upper cut1214 off point.

1215 Dispersion, as I have explained in chapter 3, is also an important aspect to 1216 control for, in order to minimise idiosyncratic uses of a bundle (one or two writers 1217 favouring a bundle that is not used by anyone else, for example) and thus each bundle 1218 needed to appear in at least five documents in the corpus to be included in the final 1219 list.

1220 Once the settings had been decided upon, a search was carried out and a list of 1221 4-word bundles produced. The second important stage, once the list was extracted, 1222 was the identification of suitable bundles for further analysis. Sketch Engine 1223 automates the search but cannot distinguish easily between 4-word sequences that are 1224 random, or part bundles. To help me identify suitable bundles a list of exclusion

1225 criteria had been drawn up and appears below in Table 16. The exclusion criteria were 1226 arrived at based on my readings of the literature.

1227 Table 16 Exclusion criteria applied to extracted list of lexical bundles

\section{Exclusion Criteria}

Fragments of other bundles i.e. eat or drink any; tip of the part

Topic/Name specific e.g in X Plain-T; University College Central Clinic

Bundles with random or meaningless numbers e.g. know page 40 if

Web noise e.g. at www.radio.com

Clear Legal disclaimers e.g. do not copy this

1228 Once the exclusion criteria had been applied, the bundles were classified 1229 according to their grammatical type using the taxonomy first described by Biber et al. 1230 (1999). 
1232 functions, using the categories described by Biber et al (1999) and expanded in Biber

1233 et al. (2004) and Conrad and Biber (2005).

1234 Assigning discourse function is not obvious simply from looking at an isolated

1235 bundle. Bundles, in fact, do not necessarily possess a function irrespective of context,

1236 and some bundles can appear in different categories as I explained in chapter 3: they

1237 possess multiple functions that are context dependent, making it imperative to

1238 investigate how the bundles are used in the data before assigning them to a category.

1239 By way of example, at the end of can be used to refer to both time and to place, e.g. at

1240 the end of the corridor or at the end of the day. It can also be used as an expression of

1241 identification or focus in a sentence such as at the end of the process. Checking the

1242 use of the bundles in the corpus, then, though a long process, is an essential one.

\section{$1243 \quad 5.4$ Results}

$1244 \quad 5.4 .1$ Overall distribution of lexical bundles in patient information

1245 In this section, I will first report on the overall frequency of lexical bundles and the

1246 distribution and frequency of their structural type. Then I will present the results of the 1247 categorisation of discourse function.

$1248 \quad 109$ unique types bundles were extracted from the corpus of 408,997 running 1249 words. There is a total of 3725 bundles, representing $3.6 \%$ of the total number of 1250 running words in the corpus. To put this figure into some comparative perspective,

1251 Goźdź-Roszkowski (2011) found that 4-word bundles represented 4.2\% of the running

1252 words in a corpus of legal textbooks, $2.4 \%$ of the running words in a corpus of

1253 professional articles and 9.4\% in a corpus of legislation. Conrad and Biber (2005)

1254 found that 4 -word bundles made up $3 \%$ of a corpus of conversation (compared to $25 \%$

1255 for 3 -word bundles), and $2 \%$ of a corpus of academic prose. $3.2 \%$, then, is a finding 1256 that seems appropriate for a discourse type that seems, at first glance, to lie midway

1257 between conversation and more formal prose. The final list of bundles, ordered by 1258 frequency, can be seen in Appendix B. 
1260 A closer look at my data reveals that as many as two-thirds of the bundles are 1261 of a structural type that is more commonly found in academic prose (Biber et al., 1262 1999). The structural types found in my corpus of patient information can be seen 1263 below, in Table 17

1264 Table 17 Distribution of structural types of lexical bundle in patient information after Biber et al. (1999)

Distribution (\%) of bundles by grammatical type in patient information corpus*

More common in academic prose

\begin{tabular}{|c|c|c|}
\hline $\begin{array}{l}\text { pronoun + lexical verb } \\
\text { phrase }\end{array}$ & 8.0 & $\begin{array}{l}\text { NP + post-modifying } \\
\text { fragment }\end{array}$ \\
\hline pronoun/NP + $(\mathrm{AUX})+$ be & 5.0 & Prep + NP fragment \\
\hline $\begin{array}{l}\text { (pronoun) }(\mathrm{AUX})+\text { active } \\
\text { verb }\end{array}$ & 16.0 & $\begin{array}{l}\text { 'it' + VP/adjP ( + } \\
\text { complement clause })\end{array}$ \\
\hline yes-no + QU-word fragment & 5.0 & Passive verb + PP fragment \\
\hline $\begin{array}{l}\text { (verb) }+ \text { WH-clause } \\
\text { fragment }\end{array}$ & 0.0 & $\begin{array}{l}\text { Verb (+ that) clause } \\
\text { fragment }\end{array}$ \\
\hline
\end{tabular}

Other expressions $\quad 6.0$

Total 34.0

Total 66.0

$1265 *$ rounded to the nearest $0.5 \%$

1266 Two structures more common to academic prose are particularly frequent in

1267 the corpus: Passive verb + PP fragment, e.g. that may be used; can be treated with;

1268 may be needed to; and Prep + NP fragment, e.g. during the course of; at high risk for. 
1269 This last type, Prep + NP fragment, are the most frequent type in the discourse and

1270 well over a quarter of all the bundles fall into this category. These two categories

1271 alone account for $45 \%$ of all of the bundles in the final list of 109 .

1272 A third of the bundles were of a kind that predominate in conversation, the

1273 most common being Pronoun + AUX + Active verb, e.g. you may feel a, representing

$127416 \%$ of the number of bundles. The high frequency of modal verbs used in patient

1275 information may well explain the predominance of this kind of bundle. We shall

1276 report on the use of modal verbs in patient information in the following chapter.

1277 These results show that the frequency of structural types varies quite markedly,

1278 with some bundle types being used repeatedly, while others appear very infrequently.

1279 Five bundle types, two more common in conversation and three more usual in

1280 academic prose, represent around $78 \%$ of the final list of 109.

$1281 \quad$ 5.4.3 Discourse function of bundles

1282 With regards to the discourse function of the bundles, the results appear below in

1283 Table 18. As we can see, the most frequent bundle types are split more or less evenly

1284 between referential and stance: 52 bundles are categorised as stance bundles while 54

1285 are categorised as referential bundles. Discourse organising bundles, on the other

1286 hand, are used far less frequently and make up just $12 \%$ of the total number of

1287 bundles.

1288

1289

1290

1291

1292

1293

1294 
Attitudinal

1299

1300

Desire

Obligation/Directive

Intention/Prediction

Ability

\section{DISCOURSE ORGANISING}

13

Topic introduction/focus

6

Topic elaboration/clarification

\section{REFERENTIAL BUNDLES}

41

Identification/focus

6

Imprecision

Specification of Attributes

Quantity specification

Tangible framing attributes

2

Intangible framing attributes

7

Time reference

Place reference

Text reference 
1304 We have seen that some bundles are used with great frequency in certain types of 1305 discourse. Conrad and Biber (2005) reported that one bundle, I don't know was used

1306 over 1000 times per million words in conversation. Such high rates of use are less 1307 common in academic prose, however.

1308 In patient information, there are very few bundles that are used with great

1309 frequency, and none at all used with the frequency of certain bundles in spoken

1310 discourse. There are five bundles used more than 200 times per million words: you

1311 may be asked, if you have any, you will be asked, if there is any and how do I get. The 1312 most frequent bundle in my data, you may be asked, appeared 221 times (471 pmw).

1313 This was followed by if you have any, which occurred 207 times (443 pmw) and you

1314 will be asked, at 186 occurrences (396 pmw). If there is any occurred 140 times (299 1315 pmw) and how do I get appeared 97 times (207 pmw).

1316 In their study, Conrad and Biber (2005) found that the most frequent bundles 1317 in academic prose appeared between 200-400 times per million words, a similar 1318 finding to that reported here for patient information. Compared to conversation, then, 1319 written patient information shows evidence of being formulaic, but it does not have 1320 the repetitive characteristics of spoken discourse. A wide variety of bundles are used, 1321 but only five bundles appear with any notable frequency - i.e. more frequently than 1322200 per million words.

1323 While patient information makes use of a number of bundle types that are 1324 more commonly found in conversation, bundle types that are more common in 1325 academic prose and informational discourse predominate. I now turn to a detailed 1326 discussion of these findings.

\section{$1327 \quad 5.5$ Discussion}

1328 In this section, I will discuss some of the more significant findings reported above. I 1329 will begin by focussing on the structural types of bundle found in the data, proposing 1330 some explanations for the reliance on these bundle types, and, with my research 1331 questions in mind, considering what the occurrence of these bundle types tells us 
1332 about the characteristics of patient information. I will then move on to investigate the

1333 discourse functions of the bundles extracted in more detail, an investigation that will

1334 further my understanding of the lexical characteristics of patient information but also,

1335 perhaps, reveal some more of its underlying discourses, some of which have been

1336 reported on in chapter 4.

1337 5.5.1 Structural types

1338 5.5.1.1 Passive verb + PP fragment

1339 We have seen that two-thirds of the bundle types in patient information are of the kind 1340 more often found in academic writing. This is surprising when one considers the need 1341 to produce healthcare information materials that are easy to read and accessible to the 1342 greatest number of people. Academic prose and easy-to-read text do not seem 1343 compatible.

We saw in chapter 1 that simplifying text to meet a certain reading age is the 1345 usual approach taken to making patient information readable. This generally involves 1346 a focus on shorter sentences, simpler vocabulary, with definitions provided for any 1347 medical words that need to be used and active sentences. An NHS guide for their 1348 information writers expresses it thus:

Various studies have shown that the average reading age of a British ADULT is between 9 and 12 years. So if you are writing a leaflet it might be an idea to get an average nine or ten-year-old to try to read and understand it! Readability is simply a measure of how easy a piece of text is to read. Readability can be calculated in lots of different ways, but basically the following applies: Short words + short sentences $=$ information that is easy to read. (NHS Scotland, 2007)

The guide also suggests that writers use the active voice and avoid passive structures, which is advice common to all communication guides. It is interesting, then, to note that the $2^{\text {nd }}$ most common bundle type in my data is Passive verb + PP fragment. Examples of passive structures in the data include:

1360 (3) It will be performed in the interventional radiology suite. You will be asked to lie on your back on an x-ray table. Monitoring equipment will be attached to you to measure your blood pressure and heart rate.

1363 (4) Baby soap may be used to wash the treatment area. 
1364 It is difficult, at first, to understand why the passive has been used in some

1365 sentences. (4) appeared in a section where the active voice was predominantly

1366 used:

1367

1368

1369

1370

1371

1372

1374

1375

1376

1377

1378

1379

1380

1381

1382

1383

1384

1385

1386

1387

1388

1389

1390

1391

1392

1393

If possible, shower instead of bathing, use lukewarm water, not hot and do not stay in the shower for long periods of time. Do not use shower gel, bath oils, and bubble bath as this may cause a skin reaction. Baby soap may be used to wash the treatment area. Pat the skin dry with a soft towel, do not rub as this may make the skin sore.

In other cases, an entire section was written using passive structures, including both 3- and 4-word bundles:

(6) To stop your bowel moving on the $x$-rays you may be given a small injection in your arm. The tube will be removed and you will be taken to the toilet.You may be asked to go into a different room for a further $x$-ray after you have been to the toilet.

The data in (6) came from information regarding a barium enema, a rather unpleasant procedure which the writers had previously referred to as a little undignified. Is the passive used here precisely because the procedure is considered unpleasant or embarrassing? I did not gather this information in the course of my thesis as it lay outside the scope of my inquiry, but it would certainly be an area worth further investigation.

In our first example, (3), the passive is used to describe what will happen during the examination. It also seems unnecessary to use the passive here, rather than an active verb plus 'we', e.g. we will ask you to lie down. The effect of the distance created by the passive is not at all reassuring but, on the contrary, cold and unfeeling. Who will do the actions referred to in the example? Almost certainly the radiographer or radiography nurse, both of whom, as we saw in the keyword analysis, are rarely named in the patient information. Would it not be more appropriate to use an active sentence with either one of these two professionals as subject, or 'we'? e.g. The radiographer will attach monitoring equipment to you...

The passive structure is often portrayed as a structure that is less clear and direct than its active counterpart and too complex for readers to process, irrespective of how and where it occurs in a text (Minton, 2013). This is an idea particularly 
common to communication guides. It is overly simplified, however. Zarcadoolas (2011) reminds us that context is key and references Coleman (1964) who showed that children comprehend passive structures in context, even when they could not comprehend the same structures in isolation. And while passive structures do require different processing skills on the part of the reader (Mack, Meltzer-Asscher, Barbieri $\&$ Thompson, 2013), the passive has an important function in spotlighting the focus of the sentence. As Minton (2013, p. 4) says,

When active-voice and passive-voice sentences are properly composed and appropriate to the context in which they are used, there are no grounds whatsoever for claiming that one voice is clearer or more direct than the other.

Minton (201, p. 5) illustrates his position by pointing out that each of the following sentences is appropriate depending on who, or what is the focus of interest. 'Columbus discovered America in 1482' (Active) and 'America was discovered by Columbus in 1482' (Passive). Leaving aside the fact that America was already populated and thus did not need discovering, the importance of clarifying the key message may well explain the use of passive bundles in patient information.

The examples we have seen in (3) and (6) foreground the experience for the patient. To the extent that patient information is written for patients to better understand what will happen to them in the radiography suite, the passive seems a more appropriate structure than the active (e.g. we will ask you to... or we will remove the tube) where the focus is on the medical professional. The sudden appearance of the passive bundle in (5) can, I feel, also be explained by the need to focus the attention on the most important piece of information in the sentence, which is the noun, the baby soap, as opposed to the baby oil, bubble bath and shower gel, none of which should be used by the patient, and as opposed to the verb use, which is the key verb in the paragraph and is presented early on. It is not new information.

With this in mind, avoiding the passive entirely may be impractical advice for patient information writers. It may also be the case that presenting information in the active voice only, when it would be more logical sometimes to present it in the passive, may have an impact on how the information is read and comprehended. In the 
1429 following two sentences (not taken from my data), the active sentence in (8) seems

1430 less clear than the passive in (7):

1431 (7) A CT scanner is used to take multiple x-ray images of your body

1432 (8) We use a CT scanner to take multiple x-ray images of your body

The active sentence also leaves open the question of whether other machines

1434 could have been used, or whether CT scanners have other functions, as well as take

1435 multiple x-ray images. They do not. Based on this one example, it would seem that

1436 active sentences are not always clearer than passive sentences.

1437 To conclude this section, further studies of how and why passive bundles are

1438 being used in patient information, and how patients feel about them in terms of ease of

1439 comprehension and clarity, would be very welcome.

1440 5.5.1.2 Prep + NP fragment

1441 What of the other bundle-type very commonly used in patient information: the Prep +

1442 NP fragment? Almost a third of the total number of bundles were accounted for by

1443 this bundle-type. Examples of this structure include at the end of, in the area of and

1444 during the course of. Investigating the bundle structure in the corpus I discovered

1445 that, while some of these structures had a framing function (e.g. as a result of), the

1446 majority of the structures referenced time and place:

1447 (9) This procedure combines special $x$-ray equipment with sophisticated

1448 computers to produce multiple images or pictures of the inside of the body.

1449 (10) The technologist will attach electrodes to your chest, wrists, and ankles. These $1450 \quad$ will be used to record an EKG at the same time the echo is taken.

1451 The reliance on these types of structural bundles underlines one of the primary

1452 functions of patient information, that of providing information. Referential bundles

1453 are, in fact, a strong feature of informational discourse (Biber et al., 2004; Biber \&

1454 Barbieri, 2007; Barbieri, 2018) In fact, information-giving appears to be the primary

1455 function of the majority of bundles in the corpus, as I will demonstrate in this

1456 discussion section. The information included the benefits of the medical procedure,

1457 patient preparation for the procedure, the steps of the procedure itself, the time 
1458 required for the procedure, any equipment used, the meaning of certain significant

1459 terms and post-procedural recovery.

1460 Referential bundles will be discussed in more detail in 5.5.2.

$1461 \quad 5.5 .1 .3$ (pronoun) $($ AUX) + active verb

1462 While structure-types were predominantly those more commonly found in academic

1463 prose, a third of the bundles are those that are more commonly found in conversation.

1464 These structures are clausal, often involving a verb or auxiliary verb. Of these, the

1465 most commonly used, representing 16\% of the 109 bundles, was (pronoun) (AUX) +

1466 active verb.

1467 Examples of this type include you will have a; you will need to and may need

1468 to be. As will see in the following chapter, auxiliary verbs may and will are very

1469 frequent in patient information, and, as might be expected, the future is often

1470 referenced. Need to is also more frequently used than expected when its use in general

1471 English is compared (e.g. Johansson, 2010). Modal verbs for giving instructions will

1472 be discussed in detail in the following chapter.

1473 Let us now turn to a more detailed look at the discourse function of the

1474 bundles. I will begin by looking at Referential bundles, which represented more than a

1475 third of the bundles extracted.

$1476 \quad$ 5.5.2 Referential bundles in patient information

1477 5.5.2.1 Specification of attributes: Time

1478 Referential bundles that identify some specific attribute of the following head noun

1479 often relate to time, place or text. In the patient information corpus, these make up

1480 more than $50 \%$ of all referential bundles. On closer inspection, these bundles are very

1481 evenly distributed between those referencing time and those that reference place.

1482 There was just one occurrence of a bundle referencing the text.

1483 The time referential bundles are nearly always imprecise. When they reference

1484 a 'window' of time they generally refer to the medical procedure itself and preparation 1485 for it: 
1486 (11) You should not eat or drink after midnight on the day of the procedure

1487 (12) The skin becomes darker during the course of radiotherapy, similar to tanning 1488 from the sun

1489 (13) ... a doctor will examine you before you leave the department.

1490 (14) ...patients should avoid blood-thinning medication for the recommended $1491 \quad$ period of time before the treatment.

1492 (15) Detailed instructions will be given at the time of booking your appointment.

1493 (16) The therapy is usually given over a period of several weeks

1494 When the bundles refer to recovery time or possible side-effects, they name 1495 the unit of time (weeks, hours or seconds) but, generally, they too are imprecise and 1496 approximate:

1497 (17) You may feel a warm sensation for a few seconds when the dye is injected

1498 (18) You may feel sore at the end of the biopsy for a few days

1499 (19) Skin reactions usually heal completely within a few weeks of completing 1500 radiotherapy

1501 The difficulty of predicting with any great certainty the duration of anything 1502 medical is a likely explanation of the imprecision. It is also the case, as we have seen 1503 in 4.5.1, that the issue of time is a legally sensitive one. A patient may feel that they 1504 have grounds for complaint or legal action if their experience does not match official 1505 information. Being vague is legally advantageous.

1506 5.5.2.2 Specification of Attributes: Place

1507 Unlike imprecise time bundles, those that reference place are relatively precise, and 1508 nearly always reference the body area being examined, or a part of the scanning 1509 machine:

1510 (20) Tissue samples are removed from the area of concern using a hollow needle 
1511 (21) It also is possible that the catheter tip will separate material from the inner lining of the artery, causing a block downstream in the blood vessel.

1513 (22) [...] the CT table moves you very slowly towards the hole in the centre of the 1514 "polo" shaped scanner.

1515 There is one bundle, at the end of, that is used to reference both place,

1516 (23) A balloon at the end of the catheter is inflated with contrast

1517 (24) Small balloon-like sacs called alveoli are at the end of the bronchial tubes

1518 and time,

1519 (25) At the end of the procedure, the applicators are removed

1520 (26) [...] ] at the end of the operation, the anaesthetist will stop giving anaesthetic

1521 drugs and you will start to wake up.

1522 The same bundle was also used once to reference the text:

1523 (27) [...] details can be found at the end of this leaflet.

1524 The focus in patient information on explaining what instrument will be acting 1525 on what body part explains the reliance on these bundles. There is more precision with 1526 these bundles as place information comes without the legal pressures that accompany 1527 time referential bundles.

1528 5.5.2.3 Specification of Attributes: Tangible and Intangible framing

1529 Framing bundles also identify attributes of the noun that follows. These attributes can 1530 be more concrete in nature (tangible) or abstract (intangible). Framing bundles 1531 represent around $10 \%$ of all bundles. Some of these bundles are used to identify and 1532 name something:

1533 (28) This radioactive material accumulates in the organ or area of your body being 1534 examined, where it gives off a small amount of energy in the form of gamma 1535 rays 
1536 (29) Follow-up imaging may be necessary to ensure that no foreign bodies remain in the body and to check for the presence of any side effects such as infection.

1538 The bundle as a result of seems to have a specialised use in the corpus, and is 1539 overwhelmingly used to refer to the side effects of a treatment or the (negative) result 1540 of a disease:

1541 (30) In P.A.D., the arteries that carry oxygenated blood throughout the body 1542 become narrowed or even blocked, usually as a result of atherosclerosis, or $1543 \quad$ plaque

1544 (31) Side effects of radiation treatment include problems that occur as a result $1545 \quad$ of the treatment itself

1546 (32) [...] there are rare reports of people having died as a result of infection 1547 thought to be due to the biopsy.

1548 Likewise, the best way to, which also seems to have a special use in the 1549 corpus, and is predominantly used to justify the proposed medical procedure or to give 1550 post-surgery/treatment advice:

1551 (33) An angiogram is the best way to find out if arteries are blocked or restricted

1553 (34) The best way to fight fatigue is to get on a daily exercise regimen that is 1554

(35) Follow-up examinations are sometimes the best way to see if treatment is working or if an abnormality is stable over time.

1558 5.5.2.4 Specification of Attributes: Quantity

1559 There were a handful of bundles that expressed a quantity, none of which were 1560 precise. This lack of precision we have seen previously with referential bundles 1561 related to time. 
1562 (36) Sometimes, one or more of these warning signs may happen and then

1563 disappear.

1564 (37) In a conventional $x$-ray exam, a small amount of radiation is aimed at and 1565 passes through the part of the body being examined

1566 (38) Some patients may cough up a small amount of blood after the procedure.

1567 The warning signs, radiation, and blood appear almost inconsequential by the 1568 use of these imprecise quantifiers, which may indeed be the objective.

1569 5.5.2.5 Specification of Attributes: Identification / Focus

1570 Finally, identification/focus bundles, are often used to name or define something

1571 medical, with the intention of clarifying things for the reader:

1572 (39) The procedure is also sometimes referred to as Uterine Artery Embolization $1573 \quad(U A E)$

1574 (40) X-rays are a form of radiation like light or radio waves

1575 The bundle any of the following is used to present a list of options, sometimes 1576 relating to existing complaints or current medication, but also to potential side effects 1577 of treatment:

1578 (41) Please inform a member of staff if you answer 'yes' to any of the 1579 following questions...

1580 (42) Please indicate if you have any of the following...

1581 (43) You should report to your physician immediately if you experience any of the 1582 following after your procedure... 
1587 Stance bundles are the most frequent bundle in the corpus, even more so than

1588 Referential bundles, accounting for around half of all the bundles used. Stance relates

1589 to the expressions of attitudes or expressions of certainty that frame some other

1590 proposition (Biber et al., 2004, p. 384); there are two main categories of stance in

1591 Biber et al.'s (2004) taxonomy, Epistemic, which relates to expressions of certainty,

1592 and Attitudinal, which includes a number of sub-categories: Intention/prediction;

1593 Obligation/Directives; Ability and Desire.

1594 Intention/Prediction are by far the most frequent, accounting for around 50\%

1595 of the total number of Stance bundles and more than $20 \%$ of the total number of

1596 bundles. Intention/Prediction bundles are followed by Ability with 13 bundles and

$159715 \%$ of the total number of bundle types, and Obligation/Directives with 9 bundles,

1598 around $10 \%$ of types. The Stance bundles seen in patient information are

1599 overwhelmingly impersonal, that is, they are not overtly attributed to the writer but to

1600 the organisation (the hospital in many cases) or the medical system itself.

\section{$1601 \quad$ 5.5.3.1 Intention/Prediction Bundles}

1602 These bundles have a clear use in patient information and that is to say what is certain

1603 or likely to happen during and after the patient's visit to the hospital, and what the

1604 patient is certain or likely to (be expected to) do. The ratio of bundles expressing a

1605 possibility (very often with the modal verb may) to those expressing a certainty (with

1606 will) is around 2:1. As we have said earlier in this chapter, predicting with certainty in

1607 the field of medicine is not straightforward and it is not surprising that may is used

1608 twice as often as will in these bundles.

1609 There are two Intention/Prediction bundles in the five most-used bundles in the

1610 corpus: you may be asked (appearing 220 times (471 pmw) and you will be asked,

1611 occurring 186 times (398pmw). Both of these bundles are in the passive form,

1612 possibly to focus on the 'you' of the patient. I have discussed this in more detail

1613 earlier in this chapter in 5.5.1.1. 
1614 The fact that these bundles appear in the category Stance is not the end of the story,

1615 however. Examining these bundles in the corpus, I understood that both you will be

1616 asked and you may be asked generally function as instructions in patient information.

1617 A variation on these two is the active form we will ask you, which appears far less

1618 frequently in the corpus at just 13 raw occurrences. Though it seems to merely state

1619 what will happen, closer inspection reveals that it too is used to reference an

1620 instruction:

1621 (44) The scan is taken very quickly and you will be asked to hold your breath whilst 1622 it is taken.

1623 (45) On arrival you will be asked_to undress in a cubicle.

1624 (46) You may be asked to change into a gown before your scan

1625 (47) You may be asked to remove any piercings, if possible.

1626 (48) We will ask you to remove all jewellery and body piercings before the scan as 1627 the scanner uses a very strong magnet.

1628 Irrespective of whether will or may is used, the information content is

1629 generally the same, suggesting that the choice of may or will in these bundles is down

1630 to the writer's preference.

1631 In other cases, the choice of will over may seems to relate more closely to the

1632 idea of something that can, with certainty, be predicted. These include events that are

1633 invariable, such as the steps a patient is expected to follow when they arrive at the

1634 hospital, or the stages of a procedure that are the same for any patient.

1635 (49) You will have a blood test at the start of treatment

1636 (50) You will be asked to lie down on an x-ray table

1637 (51) The Radiologist performing the Nephrostogram will be able to let you know 1638 the results of the test before you leave the Department. 
1639 How long something will take, however, i.e. the procedure, waiting time and

1640 recovery time, is variable, and not something that can be predicted with any great

1641 accuracy. As we have seen earlier in this chapter, nor is it something that medical

1642 bodies want to state with too much precision in case it lays them open to complaints

1643 and legal action when the reality fails to match what has been stated in writing. Will is

1644 not used here, but may or, less frequently, should. Likewise, aspects of a procedure

1645 that are only sometimes necessary or not appropriate for every patient appear with

1646 may or should:

1647 (52) For ultrasound of the aorta, you may need to avoid eating for eight to 12 hours 1648 before the test.

1649 (53) You should be able to resume your normal activities within a week.

1650 (54) This IV infusion may take up to two hours.

1651 How a person experiences a health condition, pain or the procedure itself,

1652 varies from individual to individual of course and, as a result, most references to

1653 sensation or pain or possible side effects are modified with may:

1654 (55) Occasionally, there may be some bleeding inside the breast and a bruise or 1655 swelling (haematoma) will form.

1656 (56) You may also be aware of pressure from the biopsy needle as it takes the 1657 sample.

1658 (57) When the radioactive material is injected into your arm, you may feel a cold 1659 sensation moving up your arm.

1660 In (55), the use of may along with the adverb occasionally and the vague quantifier 1661 some, contribute to reducing the likelihood - and threat - of the event described

1662 Interestingly, when the topic is the opposite, i.e. not feeling pain or discomfort, 1663 patient information writers are more certain, and will is overwhelmingly used. In fact, 1664 you may not feel appeared just once in the corpus and with reference to a symptom of 1665 diabetes. It was never used to reference a treatment or examination. 
1666 (58) You will not feel the catheter in your artery, but when the contrast material is

1667 injected, you may have a feeling of warmth or a slight burning sensation.

1668 (59) You will not feel the treatment and the machine is very quiet.

1669 Pain is subjective, as we know (e.g. Coghill, 2010), so it is interesting that patient

1670 information writers should be so certain in these contexts.

$1671 \quad$ 5.5.3.2 Ability

1672 There are 13 individual Ability bundles in the corpus, representing around $25 \%$ of the

1673 Stance bundles. Nine of these bundles involve the verb use, in all cases as a passive

1674 form, and in most cases modified with auxiliary verbs may and can:

1675 (60) A biopsy needle may be used to obtain a sample of lung tissue.

1676 (61) Several imaging tests can be used to diagnose P.A.D.

1677 (62) Occasionally, a device or plug will be used to seal over or close the hole in the 1678 artery.

1679 (63) A nephrostogram is an x-ray procedure that is used to check your nephrostomy 1680 catheter and flow of urine through your ureter (water pipe between the kidney and 1681 bladder).

1682 In patient information, the bundles that include used to always refer to physical 1683 objects - medical equipment - and to medical procedures or tests. This is in line with 1684 Durrant's (2017) finding that most bundles for the description of procedures and 1685 processes in science and technology are centred around the bigram used to.

\subsubsection{Obligation/Directive Bundles}

1687 There are nine individual Obligation/Directive bundles in the corpus, though very few

1688 are overt directives. The most frequently used 4-word bundle in this category is you

1689 will need to, the eighth most-frequent bundle overall. It appeared 49 times in the

1690 corpus (104 pmw). This bundle is used to issue instructions to the patient regarding

1691 the examination/procedure itself, 
1692 (64) You will need to stand for the treatment, holding a bar within the treatment 1693 frame.

1694 (65) You will need to have an empty bowel and a full bladder for your treatment;

1695 (66) While the camera is taking pictures, you will need to remain still for brief periods of time.

1697 or to give the patient instructions regarding the recovery period:

1698 (67) If you go home the same day, you will need to arrange for someone to take you 1699 home by car or taxi and to stay with you overnight

1700 (68) You will need to come back to the hospital for regular CT scans to check that the treatment has worked and that there is no recurrence

1702 (69) You will need to stay in hospital after the biopsy for about four hours.

1703 As I will discuss in my examination of modal verbs for instructions in the

1704 following chapter, need to structures in patient information are invariably used to refer

1705 specifically to medical procedures. While $2^{\text {nd }}$ person need to structures in other

1706 contexts are often perceived to be strong directives (e.g. 'you need to be home by

1707 midnight', uttered by a parent to a child), in patient information these structures are

1708 invariably presented as either a necessary part of the procedure ( and thus closer to

1709 dynamic necessity), or as requirements that are for the good of the patient, such as

1710 getting someone to stay over with you after returning home, or returning for regular

1711 check-ups.

1712 The most common obligation/directive 4-word bundle is it is important that 1713 which appears 90 times in the corpus, followed by the pronoun you in over $90 \%$ of the 1714 cases.

1715 (70) It is important that you follow the instruction below

1716 (71) You have been given a laxative to take before your barium enema; it is 1717 important that this is taken following the enclosed instructions 
1718 (72) These devices will be used for the treatment to achieve the same position daily,

1719 so it is important that the patient can maintain that position

1720 (73) In order to see the bowel it is important that it is empty. This is why we ask you

1721 to take the laxative prior to your scan.

1722 In each of (70)-(73), the patient is being told to do, or not to do, something, though the

1723 instruction is presented in terms of the importance of the action, leaving the patient to

1724 fully understand that this, in fact, is an instruction. In (72) and (73), why it is

1725 important is also stated, though not so in (70) and (71).

1726 The most overt directive in this category is an imperative structure, an appeal

1727 to the patient to inform the medical staff if certain conditions apply. It was unusual

1728 however, and appeared just 14 times in the corpus:

1729 (74) If you are known to have an allergy, please let us know on the day

1730 (75) If you are pregnant or think that you may be pregnant, please let us know before 1731 you have your scan.

\section{5.5.3.4 Epistemic and Desire bundles}

1733 While stance was the category of bundle most frequently used, neither epistemic or 1734 desire bundles, a subset of atttitude bundles, are included in this. In fact, there are only 1735 two desire bundle types in the entire corpus and just four epistemic types.

1736 With regards to the two desire bundles extracted in the analysis, they are both

1737 used to offer further help or information to the patient:

1738 (76) If you would like information about any medication you may be given during 1739 the scan please contact us or speak to the radiographer when you attend for 1740 your appointment.

1741 (77) If you need any assistance with transport to the hospital please contact your $1742 G P$. 
1743 Two of the epistemic bundles contain overt references to risk and chance and

1744 all came from one (American) website (www.radiologyinfo.org). It is possible that

1745 one individual was responsible for writing all or much of the patient information on

1746 this site and thus the two examples below might well be examples of idiosyncratic

1747 use:

1748 (78) Individuals at high risk for developing colorectal cancer should be screened

1749 more often and begin screening before age 50.

1750 (79) There is always a slight chance of cancer from excessive exposure to 1751 radiation.

1752 That there were so few examples of bundles relating to likelihood or risk does 1753 not mean that information related to these aspects are not presented in patient

1754 information. Risk is frequently used, appearing nearly 1,000 times in the corpus $(2,118$

1755 times per million words). Its most common collocates are possible and potential,

1756 followed by high, slight and small. Benefit, on the other hand, appears with a third of

1757 the frequency at 342 times (732 pmw) and with only one collocate that appears more

1758 than twice: potential.

1759 This finding suggests that risk is presented in radiography patient information 1760 as something that is gradeable and quantifiable, unlike benefit. A lexical analysis of 1761 the use and connotations of risk and benefit in medical information for radiography, 1762 and how patients understand these messages, would be very useful, particularly in the

1763 light of the studies I presented in chapter 2, showing that radiography patients

1764 consistently under-estimate the concomitant risk associated with certain radiography

1765 procedures and seem equally uninformed about which procedures involve radiation,

1766 and thus risk. (Singh et al., 2017; Ukkola et al., 2017)

1767 The final category of bundle to be discussed in the chapter are Discourse

1768 organising bundles, which, as we shall see in the next section, were suprsisingly

1769 infrequent in the corpus. 


\subsubsection{Topic Introduction/Focus Bundles}

1772 Discourse organising bundles are the least used bundle-type in patient information,

1773 representing just $12 \%$ of the total proportion of bundles. They are evenly split between

1774 Topic Introduction/Focus and Topic elaboration/clarification. In spite of their

1775 infrequency, however, three discourse organising bundles are among the five most

1776 frequent bundles in the corpus: if you have any; if there is any and how do I get,

1777 though as we shall see, these bundles are often used to frame instructions.

1778 If you have any is the second most frequent bundle in the entire corpus,

1779 occurring 207 times (443 pmw). This bundle is a topic introduction or focus bundle

1780 which is overwhelmingly used with the object allergies or questions/queries. In both

1781 cases, the information that follows is generally presented as an instruction. The

1782 surrounding text usually contains an imperative or a modal verb of obligation:

1783 (80) If you have any of these warning signs, call 911 right away.

1784 (81) You should tell the radiographers if you have had an allergic reaction to 1785 iodine or contrast dye in the past or if you have any other allergies

1786 (82) If you have any queries please telephone 02073518220

The next most-frequent Topic Introduction/Focus was if there is any,

1788 appearing 34 times in the corpus. On closer inspection, it transpired that all but one of

1789 these uses appeared in the American materials. While the bundle appeared in 25

1790 different documents, the fact that one author may have been responsible for writing

1791 much of what appears on the site, or that a 'house-style' may have been in use, cannot

1792 be ruled out.

\section{5.5.4.2 Topic Elaboration/Clarification}

1794 These bundles precede more detailed information about an already-introduced topic.

1795 In patient information, this can refer to the steps involved in a medical procedure,

1796 (83) How do I get the results of my scan? 
the reasons for something procedural,

1798 (84) Many imaging tests are not performed during pregnancy so as not to expose the fetus to radiation.

1800 (85) A chest $x$-ray will be taken to make sure that the lung has not collapsed from an air pocket created during the procedure

1802

(86) In a biopsy, a small amount of tissue is removed under local anesthesia so that $1803 \quad$ it can be examined in a laboratory

1804 or an explanation of an imaging modality:

1805 (87) Positron emission tomography (PET) is a type of nuclear medicine scan that 1806 uses a small amount of radioactive material to image body functions

How do I get is a particularly frequent bundle in patient information and is

1808 almost always used in the context of scan results. This is one question that

1809 radiographers field on a daily basis, underlining the importance to patients of knowing

1810 what the scan or x-ray has seen. The job of a radiographer, however, is usually

1811 restricted to carrying out radiography. Aside from specialist radiography roles which

1812 permit some diagnosing, it is the radiologist who diagnosis. Radiographers report

1813 being asked continually, sometimes pressurised, to give results by worried patients,

1814 but they are not permitted to do so. Nor, in many cases, do they possess the skills. The

1815 How do I get my results? section in patient information serves to inform patients prior

1816 to coming to the department that their radiographer will not be diagnosing them.

1817 Whether patients read this or fully understand this, we do not know.

1818 The discourse organising bundle if you do not is often used to present what 1819 will or could happen if the patient acts in a manner that is contrary to that advised or 1820 desired by the hospital. Sometimes this presented in a manner that is quite alarming, 1821 as in (88).

1822 (88) If you do not follow your diet, exercise, and perform sugar level tests, serious $1823 \quad$ complications can arise.

1824 (89) If you do not get treatment, chest pain may happen more often. 
1825 Discourse organising bundles are used far less frequently than might be

1826 expected, given the nature of the text. On the other hand, we have seen that three of

1827 these bundles are, in fact, used very often in the text, with three in the first-5 most

1828 frequent. This demonstrates that while there is little variety in the bundles, a small

1829 number of them are relied upon and have an important function in patient information.

1830 Discourse organising bundles 'reflect relationships between prior and coming

1831 discourse' (Conrad et al; 2005, p67) and serve to introduce a change in topic or to add

1832 more detail to the topic being discussed. It may be that the style and format of much

1833 patient information render discourse organising bundles less necessary. Patient

1834 information is often arranged as a series of questions and answers - and some of the 4-

1835 word, discourse organising bundles in patient information are part-questions - e.g.

1836 when will I get; why do you need.

1837 All advice to patient information writers to dispense with long sentences and 1838 complex structure should mean a minimal number of complex paragraphs that require

1839 a range of connecting, cohesive devices. The following paragraph is typical of the 1840 patient information in the corpus. There are ten sentences but no relative pronouns.

1841 The average sentence length is 15 , though the shortest sentence is just six words and

1842 the longest is 24. The paragraph contains no 4-word, discourse organising bundles

1843 (other 4- and 5- words bundles underlined):

1844 Bronchoscopy The doctor uses a bronchoscope during bronchoscopy. A

1845 bronchoscope is a long, thin, and flexible fiber optic tube that transmits pictures from

1846 the tip to an eyepiece or to a video set. During a bronchoscopy, the bronchoscope is

1847 used to look at the larynx, trachea, and bronchial airways of the lungs. This

1848 procedure shows more details from the inside of the airways than pictures taken with

1849 X-rays. The bronchoscope has an open channel. This allows instruments to go through

1850 the scope and be used to take tissue samples, cauterize bleeding, or remove thick

1851 mucus blocking the airways. The doctor that performs the bronchoscopy procedure is

1852 a pulmonologist, $\underline{\text { a specialist in the respiratory system. A bronchoscopy can be used to }}$

1853 examine many different respiratory tract symptoms. These include pain in the trachea,

1854 difficulty breathing, bleeding, tumors, and chest pain. Clear and detailed images and

1855 video projected on a monitor helps the doctor diagnose problems. 

paragraph would look with the addition of a discourse organising bundle or two. It

1858 may be that the simplification of the text, the shorter sentences and the repetition of

1859 the nouns, in place of pronouns, perform the functions ascribed to discourse

1860 organising bundles, that of topic focus, elaboration and clarification. Some targeted

1861 experiments would help answer that question. The text would look differently with

1862 some relative pronouns too (i.e. that, which, who), of which there are none. The

1863 sentence the doctor that performs the bronchoscopy procedure is a pulmonologist, a

1864 specialist in the respiratory system could also be written as the doctor that performs

1865 the bronchoscopy procedure is a pulmonologist, who is a specialist in the respiratory

1866 system. Which would patients find easier to read?

1868 should, in my view, also be looking at cohesion and coherence, which discourse

1869 organising bundles contribute to, and not only at vocabulary clarity and complexity.

1870 Zarcadoolas (2011), discussed in chapter 2, cites Redish and Seizer (1985) and Ancker 1871 (2004) in saying:

Often the mandate to write or revise text to meet formal readability criteria leaves writers and materials developers in a Catch-22, and can result in actually trying to game the system by artificially dividing sentences and using sentence fragments. Adding the very words or sentence types that would make the text more com-prehensible unhappily increases the readability score of the material, and thus is judged inappropriate. (p343)

\subsection{Conclusions}

My analysis has shown that four-word lexical bundles appear frequently in patient information, though none are used with anything like the frequency seen in conversation, where the most frequent individual bundles appear over 1,000 times per million words (Biber and Conrad,2005). In patient information, the most frequent bundles appear between 200 and 400 times per million words, a similar rate to that found in academic prose (Biber and Conrad, 2005). Similarities with the bundles found in academic discourse are also seen in the structural types of bundles in patient information. Two-thirds of these bundles are of the kind found more often in academic writing, and almost a third of the total number of bundles were accounted for by just 
1888 one structure: Prep + NP fragment (e.g at the end of). With regards to the discourse

1889 function of the lexical bundles, they are split between referential and impersonal

1890 stance bundles, the latter predominating.

1891 Referential bundles are frequent in academic discourse but also in

1892 informational discourse. It is the informational content in patient information that

1893 explains the preponderance of bundles that are common to academic prose. Indeed,

1894 the primary function of the majority of bundles in the corpus appears to be that of

1895 information-giving. In patient information, there is a premium put on transmitting

1896 practical information relating to the procedure, and on the patient's experience at the

1897 hospital. And where there is a precision seen in many of the referential bundles,

1898 particularly those that relate to place, many of these bundles are often uncertain:

1899 modified by may, or with imprecise temporal terms which may well be explained by

1900 the fact that a patient's experience of a medical intervention is highly individual, and

1901 the daily workflow of a hospital environment unpredictable.

1902 It is also true that avoiding certainty in patient information may confer some

1903 legal protection for the hospital or healthcare system. This imprecision is seen in the

1904 Stance bundles too, many of which relate to Intention and Prediction. Interestingly,

1905 some of these Stance bundles are, in fact, functioning as instructions. You will be

1906 asked and you may be asked are two examples, and as we have seen, they are also the

1907 second and third most-used bundles in the entire corpus, highlighting the importance

1908 of instruction, alongside information, in patient materials.

1909 And while instruction is one of the two primary functions of patient

1910 information, many of the bundles that function as obligations are not direct and do not

1911 come from the Obligation/Directive category. We have seen, too, that while

1912 Obligation/Directive bundles are relatively common in patient information, the vast

1913 majority of these bundles are not direct either. In fact, directness in patient

1914 information seems to be something to be avoided, particularly when telling the patient

1915 what to do. In my corpus, the majority of bundles in the Obligation/Directive category

1916 involved the use of need to and, to a lesser extent, should. These two modal verbs,

1917 along with others used to instruct, are the subject of the third analysis, reported on in

1918 the next chapter. 
Indirectness is also achieved in patient information for radiography by using

1920 an impersonal structure such as it is necessary to or it is very important. The question

1921 is raised, however, of how a phrase like it is important to are perceived and

1922 understood by patients, for whom the reading 'important for others but not me' is

1923 always a possibility.

1924 In terms of lexical characteristics, the lexical bundles analysis has provided

1925 clear evidence of the primary communicative concerns of patient information:

1926 information and instruction. We have seen, too, that the bundle types that are more

1927 often found in conversation, (pronoun) (AUX) + active verb, often contain modal

1928 auxiliary verbs such as may, and many references to time or experiences in the patient

1929 information are vague or imprecise. The pronoun used in the majority of the cases of

1930 this bundle type is 'you'.

1931 As discussed in Chapter 1, The NHS in the UK produces a guide to writing

1932 patient information and the use of you and we are encouraged. Bundles in patient

1933 information, though, very rarely make direct reference to the hospital or the medical

1934 system, and very rarely do they include we. The pronoun you appears 6,544 times

1935 (14,014.38 pmw), while we appears just 326 times (698 pmw). Some documents never

1936 use we at all. There are just two 4-word bundles in my analysis, we may have to and

1937 we will ask you, that use we, against 31, 28\% of the total number of 4-word bundles,

1938 that use you. The focus, then, seems very much to be on the patient. This focus on the

1939 patient was also seen in the number of bundles that use a passive structure, putting the

1940 patient in the initial position, e.g. you will be asked instead of we will ask you. You

1941 may be asked and you will be asked are the first, and the third, most frequent 4-word

1942 bundle in my corpus. This finding seems as odd with the advice to patient information

1943 writers to avoid the passive, and I wonder whether the desire to focus on the patient at

1944 all costs may, unwittingly, be forcing the use of passive structures when an active

1945 structure, with we, would be a more natural choice.

1946 This concludes my chapter on lexical bundles in patient information. I now

1947 focus my attention on one of the two primary functions of patient information, that of

1948 obliging and instructing the patient. How this is achieved through the use of modal

1949 verbs is the subject of the third analysis, reported in the following chapter. 
1951 The purpose of patient information produced for radiography is generally twofold: to

1952 instruct and to inform. Patients, ideally, are given useful information about the

1953 medical procedure, told what might or will take place during the examination and,

1954 additionally, are told what is required or desired by the hospital before, during and

1955 after the procedure. (Patient Information Forum, 2013; Tutty \& O'Connor, 1999).

1956 In the analysis of lexical bundles in the preceding chapter, a number of bundles

1957 that function as instructions were revealed, e.g. you will be asked to and it important

1958 that. We saw, too, in chapter 4, that please is a keyword in patient information, and is

1959 used to preface an imperative, e.g. please go to the main hospital reception desk.

1960 Inviting contact is one of the uses of this structure, while its other principal use is to

1961 instruct the patient. Another finding from the lexical bundle analysis of relevance here

1962 is the frequent use, in patient information, of various structures with need to and

1963 should. Need to is classed as a semi-modal, and should a modal verb.

1964 How these words are used in patient information, with what frequency and with what

1965 effect is the focus of this chapter, as is the use of the other modal verbs and semi-

1966 modals that are used in English to give instructions.

1967 I will begin by explaining the role and importance of instructions in patient 1968 information in general, and my reasons for selecting modal verbs as the subject of my 1969 analysis. This will be followed by a section that presents an overview of modal verb 1970 meaning and some findings from the literature regarding frequency and use in different 1971 varieties of English. The methodology, which has been presented in full in chapter 3, 1972 will be summarised before I present my results and a discussion of those results. Let us 1973 begin by considering the role of instructions, and the importance of following 1974 instructions, in patient information.

\section{$1975 \quad 6.1$ The importance of instructions in patient information}

1976 When instructions are not followed, the patient is often said by medical professionals 1977 to be exhibiting 'non-compliance' or 'non-adherence'. The terms refer to two different 
1978 kinds of behaviour, the latter suggestive of unintended consequences, the former more 1979 complex and intentional behaviour (Jones, 2013).

This is an important and much-researched healthcare topic which, while it is

1981 outside the scope of my research, is of great relevance when we consider the role 1982 language may have in explaining why patients intentionally, or unintentionally, fail to 1983 follow instructions.

1985 patient compliance (I will use the term compliance to include adherence in this

1986 chapter), demonstrated just how complex and varied the reasons are. Jin et al. (2008)

1987 identified as many as 25 factors that could affect compliance, though conflicting

1988 results in different studies they looked at suggest that the factors governing an

1989 individual's ability or willingness to comply are very complex.

Where study results were unequivocal, however, was in the area of the patient1991 provider relationship and communication. Studies consistently show that patients are 1992 far more likely to exhibit compliance when they feel that they are being treated as an 1993 equal partner, when there is empathy from a provider, and where patients exhibit 1994 higher levels of trust towards their provider (Jin et al., 2008, p277). Feeling informed 1995 contributes to this feeling of trust, and how medical professionals communicate with 1996 their patients also contributes to this trust and to the experience of being treated as an 1997 equal partner. In ideal patient-centred care, a patient needs to feel confident that they 1998 have a voice and that decision-making power is being held by both parties. (Patient 1999 Information Forum, 2013). Language is pivotal to the development and maintenance 2000 of this relationship, and this relates to both spoken language, as in a consultation or 2001 written language, as in a patient information leaflet.

2002 People are told what to do by other people very frequently in certain settings:

2003 in the workplace, school and in healthcare interactions particularly, and this is a well2004 researched area in sociolinguistics (e.g. Holmes and Stubbe, 2003; Vine, 2004). Most

2005 of this research has focused on spoken interaction, while the language used for 2006 instructions and obligations in written registers has received scant attention in the 2007 literature. This absence in the literature is a motivating factor for me to focus on the 2008 instructions and obligations in patient information. 
2009 In the next section I explain why I elected to focus particularly on modal verbs for

2010 instructions, rather than any other means of instructing, such as imperatives.

\section{6.2 Why modal verbs?}

2012 Overt instructions seem to be largely absent in patient information for radiography, 2013 based on my two analyses thus far: keywords and lexical bundles. My analysis of the 2014 latter, presented in the previous chapter, suggests that aside from an imperative with

2015 please, direct obligations and bald directives are not at a feature of the register.

2016 Appeals to the importance or necessity of something, e.g. it is important to, and it is 2017 necessary to are preferred, and I also found a number of bundles making use of need 2018 to. This semi-modal verb, along with the modal verb should, appeared in a number of 2019 very frequently used bundles, although their stronger counterparts, i.e. must and have 2020 to, did not. While the findings from my lexical bundle analysis has suggested this is 2021 an area worth further investigation, a targeted analysis will give us more detailed 2022 information.

2023 Another reason for investigating modal verbs in patient information is that 2024 modal verbs are very common in medical writing in general. This is particularly true 2025 of epistemic modals, which are often used in hedges or boosters in research papers 2026 (e.g. Salager-Meyer, 1994), and of modal verbs of obligation, particularly must and 2027 should which have been found to be frequent in a number of medical registers, 2028 included case notes and editorials (Vihla, 1999). Vihla (1999) did not include patient 2029 information in her study of a range of modals in medical registers, however, and as I 2030 have stated already, I am not aware of any studies that have looked at instructions in 2031 patient information, or at modal verb use generally in patient information. The 2032 investigation described in this chapter is a response to some of this gap in the 2033 literature

In the next section I will present a summary of modal and semi-modal verbs in 2035 English, describing their range of meaning, their use in different modes and varieties 2036 of English, and the changes in use that have been documented by applied linguists. 
2038 Modality is the expression of possibility or necessity, and in English can be expressed 2039 by many means including modal verbs, semi-modal verbs, adjectives, nouns, adverbs, 2040 and particles. For the purposes of this study, the categories of auxiliary modal verb 2041 (also called central or core modals and one of the most common means to express 2042 modality) and semi-modal verbs (Palmer, 1983, p208) are considered.

2043 The core modals are generally held to be can, could, may, might, shall, should, 2044 will, would, ought (to) and need (Downing and Locke, 1992; Quirk et al., 1985) while 2045 the category of semi-modals can include a range of items including dare to, need to, 2046 have (got) to, be able to and be going to. Semi-modals express meanings that can 2047 usually also be paraphrased with a core modal, e.g.I have to lose weight, and I must 2048 lose weight. (Biber et al., 1999). Some semi-modals, unlike modal verbs, can be 2049 marked for tense and person, e.g. have (got) to.

2050 The main functions of modal and semi-modal verbs (henceforth modals) is to 2051 express stance. (Biber, Conrad \& Leech, 2002). Modal meaning is usually categorised 2052 as epistemic, deontic and dynamic, though Biber et al. (1992) propose three other 2053 names for the categories: permission/ability; obligation/necessity and 2054 volition/prediction. Epistemic, deontic and dynamic categories of meaning I will 2055 present in 6.4.1.

2056 The literature on modal verbs, their meaning and use, is huge and beyond the 2057 scope of this study, however. I am interested in how a small selection of modal verbs 2058 from one category of meaning (deontic) are used to give instructions in patient 2059 information, though some background information regarding meaning and modals is 2060 necessary for the sake of clarity. To this end, I will present an overview of modal 2061 meanings in the following section, with particular emphasis on the category of 2062 meaning under investigation. 
2064 As we have seen in the preceding section, modal meanings fall into three categories:

2065 epistemic, deontic and dynamic. I will summarise epistemic and dynamic modal 2066 meaning first, before moving on to a more detailed consideration of deontic modality.

\section{6.3.1.1 Epistemic modality}

2068 Epistemic modality is concerned with the speaker's attitude towards the proposition or 2069 the situation described in the proposition. This can range from an expression of doubt 2070 through to certainty. It is concerned with '...the speaker's assumptions or assessment 2071 of possibilities and, in most cases, it indicates the speaker's confidence (or lack of 2072 confidence) in the truth of the proposition expressed' (Coates, 1983, p18). Modals 2073 commonly used to express epistemic modality include may and might. E.g. He may be 2074 the right man; it might be the right decision. Studies of hedges and boosters in 2075 medical academic writing (e.g. Salager-Meyer, 1994; Skelton, 1997) are concerned 2076 with epistemic modality. Lexical bundles can also have an epistemic discourse 2077 function, as we saw in chapter 5.

\section{6.3.1.2 Dynamic modality}

2079 Dynamic modality is less straightforward to characterise. Will, would, can, shall and 2080 be going to appear in this category and, broadly speaking, dynamic modality refers to 2081 ability or volition - though it, unlike deontic and epistemic modality, is not subjective 2082 (Palmer, 1990, p36) which suggests to some that it is not inherently modal. Gisborne 2083 (2007) says that can, when used dynamically, 'is not a modal meaning, but rather is 2084 simply the retention of an earlier sense which persists after CAN has joined the modal 2085 verb system of English (with similar arguments applying to WILL)' (2007, p45).

\subsubsection{Deontic modality}

2087 Deontic modality is concerned with obligation, requirement and necessity. It is this 2088 category of modal that is the focus of this chapter. As we have seen, no studies have 2089 looked at the linguistic mechanisms of instruction and obligation in patient 2090 information, in spite of instruction being one of the two primary functions of patient 2091 information. English has a particularly wide range of deontic modal and semi-modals 
2092 from which to choose, all of them evidencing different collocational behaviour and

2093 different connotations, including must, should, need, need to, have to, have got to,

2094 ought to, have to and allowed to. We have already seen a suggestion, in the finding of

2095 the lexical bundle analysis, that patient information may prefer need to and should

2096 over other deontic modal verbs. A targeted modal verb analysis will explore this

2097 finding more fully.

2098 Deontic modality receives far less attention in the literature than epistemic

2099 modality, and when it does get any attention is, say Nuyts et al., (2005) it is "nearly

2100 exclusively as a 'byproduct' in the context of analyses of the formal category of the

2101 modal auxiliaries' (p. 7). This imbalance of attention is another reason for

2102 investigating deontic modal verbs in my corpus.

2103 Vihla (1999) uses the term 'performative' to refers to the function of deontic 2104 expressions (including modals) saying that 'when using them, the speaker permits,

2105 demands, or forbids something, and they can be used prescriptively to create norms of

2106 action' (p18). In the context of patient information, these norms of action might relate

2107 to behaviour around diet, lifestyle, drug or alcohol use, and equally to the behaviour

2108 expected in the context of a radiographic examination, i.e. to wear or not wear certain

2109 types of clothing, to eat or drink appropriately prior to an exam, and to inform the

2110 medical staff if pregnancy is suspected or allergies known about. The relationship of

2111 deontic expressions to norms of action had previously been stated by von Wright

2112 (1983), who says that deontic expressions 'imply the existence of an authority having

2113 the power to say what is right or wrong, i.e. 'norm authority' (p68). The authority of

2114 the speaker over the addressee is a 'felicity condition' for deontic expressions, says

2115 Vihla (1999) if the authority does not exist the utterance is not regarded as a valid

2116 command, request or permission (Vihla 1999, p18).

2117 The notion of authority is pertinent to this study. Patient information produced

2118 by hospitals and healthcare trusts exists to inform and instruct. Giving or denying

2119 permission to the patient to act in a certain way, telling the patient what to do and

2120 what is acceptable or otherwise are its primary functions. The authority in patient

2121 information can be the hospital named in the patient information leaflet, or a more 
2122 generic authority, that of the medical system, of which the named hospital and its staff 2123 are a part.

2124 Patient-centred medicine, however, has, as an objective, a rebalance of the 2125 power relations between provider and patient. Shared-decision making means, in 2126 theory, both patient and provider possess the authority to command, request or grant 2127 permission. According to Lindstrom and Weatherall (2015), both professionals and 2128 patients have what they refer to as 'deontic authority': medical professionals have the 2129 right to propose courses of treatment and behaviour while patients have the right to 2130 refuse to comply. Both epistemic and deontic authority plays a fundamental role in 2131 medical interactions and are 'complex and powerful structural forces scaffolding 2132 doctor-patient interactions and the ways treatments are recommended and responded 2133 to.' (Lindstrom \& Weatherall, 2015, p51). While Lindstrom and Weatherall's work 2134 focuses on face-to-face consultations, written patient information is also concerned 2135 with recommending, proposing and outlining medical treatments and procedures and 2136 thus is very likely to demonstrate deontic authority. Quite how much deontic 2137 authority it demonstrates, and how this authority is realised linguistically is the 2138 objective of my analysis.

2139 The results of the lexical bundle analysis presented in the previous chapter 2140 suggests that the authority of the hospital, the professional and medical system is not 2141 visible: only three lexical bundles that reference the authority using the pronoun 'we' 2142 were found, out of a total of 109 bundles. Bundles containing 'you' were 10 times as

2143 frequent. In the corpus itself, the pronoun 'you' predominates; it is used around 20 2144 times more often than 'we' (6,544 occurrences of 'you' against 326 for 'we'). The 2145 question of how deontic authority can be expressed when the identity of the authority 2146 is unclear is a pertinent one.

2147 We will return to the discussion of authority later in this chapter but let us now 2148 return to the subject of modal meaning. We have seen that modals can possess 2149 epistemic, dynamic or deontic meaning. Additionally, modals can also be used with 2150 two different types of meaning, which can mean modals can appear in different 2151 categories depending on the type of meaning being expressed. These types of 2152 meanings are usually referred to as personal (intrinsic) and logical (extrinsic). 
2154 Personal (intrinsic) and logical (extrinsic) are two types of meaning that most modals

2155 possess. Personal (intrinsic) refers to the control of events and acts by human agents,

2156 with intention, volition, obligation and permission meanings. Logical (extrinsic) refers

2157 to the logical status of states or events. Logical modal meanings are necessity,

2158 certainty or likelihood (Biber et al., 2002, p. 176). The structure of the clause can

2159 usually indicate what meaning is being expressed by the modal. Personal or intrinsic

2160 meanings have two characteristics: the subject of the verb phrase is usually human,

2161 while the main verb is dynamic and references an event or activity that can be

2162 controlled. (Biber et al., 2002). You can't sit there and John should ask for a raise are

2163 examples of personal/intrinsic meanings. Logical meaning, on the other hand, usually

2164 has a non-human subject and/or a main verb that express states: The photocopier can

2165 be found on the ground floor and That chicken should be done now are examples of

2166 modal verbs used with dynamic meaning.

2167 The deontic modals that I will be discussing in this chapter may be used with a 2168 personal meaning (obligation) or a logical meaning (necessity). As we have seen,

2169 English has a number of modals that can be used to tell people what to do: must,

2170 should, have to, need to, etc. Sometimes, of course, these modals are presented in the

2171 negative, when people are told what they cannot do.

2172 When we talk about instructions and getting people to do things, the term

2173 'directive' is sometimes used, particularly in studies from the fields of discourse

2174 analysis and pragmatics. I have chosen not to use the term, and in the following

2175 section I present an explanation of why.

\section{$2176 \quad 6.4$ Instruction, obligation or directive?}

2177 In speech act theory, a directive refers to an utterance that is used to get the addressee

2178 to do something. A directive can take many different forms, including that of a

2179 request, an invitation, a challenge, a threat and a direct obligation. Sometimes, a

2180 combination is possible. You must eat with us can be an invitation to dinner or be a

2181 direct obligation, most likely from a parent to child, perhaps: No! You can't eat in

2182 front of the TV; you must eat with us at the table. 
We have already seen lexical bundles used in patient information that are

2184 classed as intention/prediction stance bundles but, on closer examination, are revealed

2185 to be functioning as instructions. You may be asked and you will be asked, the most

2186 frequent and the third-most frequent bundle in the corpus, are not referring to

2187 questions, or requests for information, e.g. you will be asked about your hobbies/what

2188 you want for dinner, but are used to refer to instructions that will be given once the

2189 patient is in the radiography department: you will be asked to remove your clothing or 2190 you may be asked to drink a liquid.

2191 Notwithstanding the fact that many of the instructions I refer to in my data are

2192 also directives, to avoid confusion - for I am not directly referring to speech act theory

2193 or pragmatics in my study - I will refer to instruction or obligation when referring to

2194 an utterance that functions as an obligation, requirement or instruction, while deontic

2195 will be the term used for the modal or semi-modal that is used to express the

2196 obligation. I accept that instruction and obligation are not always the same thing, but

2197 both function to tell someone what to do. It is the telling-someone-what-to-do that

2198 interests me, though, for the sake of brevity, I refer to obligation or instruction in the 2199 text.

\section{$2200 \quad 6.5$ Frequency of modals in English}

2201 Dispersion and the frequency of a lexical or grammatical feature has long been held to 2202 be an important predictor of register variation (Biber 2012). Comparing modal verb

2203 frequency information from the literature with their frequency in patient information 2204 is a first step to describing the characteristics of the register.

2205 Studies show that will, would, can and could are the most frequent modals in 2206 written English (Biber et al., 2002; Kennedy, 2002; Leech et al., 2009). Kennedy 2207 (2002) suggests these four modals account for as much as $72.6 \%$ of the modals in 2208 written English. Leech et al. (2009) found that in spite of an overall reduction in the 2209 number of modals being used in written British and American English between 1961

2210 and 1991, would, will, can and could (in that order) were still the most commonly 2211 used. 
2213 in spoken language than in written academic prose. That semi-modals feature so much 2214 in spoken language is possibly less surprising than the fact that central modals also do 2215 as researchers had long thought the latter were more common in writing (Biber, 2002, 2216 p177). Not all modals are more common in spoken language, however. May is 2217 considerably more frequent in academic prose than in conversation (Biber, 2002, p.

2218 177) while must and should are found slightly more frequently in academic prose.

2219 It is worth remembering, however, that deontic modals are less frequent overall 2220 in general language than common epistemic and dynamic modals (e.g. may, can, will). 2221 Collins (2009) points out that only in the deontic category are semi-modals 2222 increasingly more frequent than core modals, evidence he says that semi-modals 2223 (referred to as quasi-modals) are 'regularly replacing their auxiliary counterparts'. (p. $222433)$.

2225 I will now present frequency information from the literature for deontic modals. 2226 I do not present detailed frequency information for epistemic or dynamic modals as 2227 they are not the focus of my study.

\section{6.5.1. Frequency of deontic modals}

2229 The reported frequency of deontic modals varies across different corpora, depending 2230 on the mode of discourse (e.g. written or spoken) and the language variety (e.g. British 2231 English, Australian English, etc.). Generally speaking, must and have to appear with 2232 far greater frequency than need to and have got to, with the use of have to equalling or 2233 surpassing that of must in spoken corpora. The status of must as a direct obligation and 2234 the need to avoid a face-threatening act renders it unsuitable for most situations in 2235 spoken language. Collins (2009) investigated modal verbs from all categories of meaning in 2237 three varieties of English: British, Australian and American. The corpora of 1 million 2238 words for British and Australian English were made up of spoken and written 2239 material, with a range of registers. The material was collected in the first half of the 2240 1990s. The US corpus was smaller in size though contained a close match of 2241 document types. The figures in brackets relating to the US corpus are adjusted 
2242 frequencies (to per million). His results of a frequency analysis of deontic modals can 2243 be seen below in Table 19.

2244 Table 19 Deontic modal frequency in GB, Aus and US English ( Collins, 2009)

\begin{tabular}{llllll}
\hline & & ICE-AUS & ICE-GB & C-US & TOTAL \\
\hline Modals & must & 613 & 675 & $402(79)$ & $1,690(1,367)$ \\
& should & 1,141 & 1,124 & $850(167)$ & $3,115(2,432)$ \\
& ought to & 36 & 80 & $51(10)$ & $167(126)$ \\
& need & 19 & 34 & $15(3)$ & $68(56)$ \\
\cline { 2 - 6 } & Total & 1,809 & 1,913 & $1,318(259)$ & $5,040(3,981)$ \\
\hline Quasi- & have to & 1,311 & 1,244 & $1,385(272)$ & $3,940(2,827)$ \\
modals & have got to & 332 & 339 & $173(34)$ & $844(705)$ \\
& need to & 343 & 280 & $473(93)$ & $1,096(716)$ \\
& had better & 48 & 33 & $41(8)$ & $122(89)$ \\
& be supposed to & 47 & 99 & $127(25)$ & $273(171)$ \\
& be to & 135 & 221 & $76(15)$ & $432(371)$ \\
& be bound to & 9 & 17 & $5(1)$ & $31(27)$ \\
\cline { 2 - 6 } & Total & 2,225 & 2,233 & $2,280(448)$ & $6,738(4,906)$ \\
\hline Total & & 4,034 & 4,146 & $3,598(707)$ & $11,778(8,887)$ \\
\hline
\end{tabular}

I will discuss some of the changes in modal use over time in the next section, 2247 and we must bear in mind that the materials contained in the corpora investigated by 2248 Collins (2009) date to the early 90s, but, nonetheless, Collins's study produced some 2249 very interesting and useful findings. The table shows a strong preference for certain 2250 modals over others, in all varieties of English. The most frequent in all varieties is a 2251 semi-modal: have to. This is closely followed by should, which appears to be more 2252 frequent in both British and Australian English (though the corpus of US English was 2253 considerably smaller in size and frequencies are adjusted.) The frequency data 2254 relating to must and need to are particularly interesting, and as we shall see, of 2255 relevance to my analysis of patient information. Must is used with more or less equal frequency in British and Australian 2257 English. In US English, however, must is used marginally less often than need to. In 2258 the GB and AUS data, need to is used, but at a similar rate of frequency to have got to, 2259 and half as frequently as must. Language change is often seen in US English before 2260 appearing - if it appears at all - in other varieties of English. Collins's (2009) findings 2261 seen in Table 18 suggests that must has been eclipsed by have to in all three varieties 2262 of English, and in the early 1990s was in the process of being replaced by need to in 2263 US English. More about the changes in modal use in the US are reported in the next 2264 section. 
2266 Our use of modal verbs has changed over time and continues to change. Johansson

2267 (2010) used the COCA (Corpus of Contemporary American) corpus (Davies, 2009) to

2268 investigate changes in must, have to, need to and have got to from 1990 through to

22692008 , providing, perhaps, some of the answers to the questions raised by Collins's

2270 (2009) study. Johansson (2010) confirmed that must is gradually falling in frequency,

2271 while have to, the most frequent deontic modal by far also seems to be dropping off.

2272 Need to, on the other hand, is rising steadily in use.

2273 I used the COCA corpus to look at the use of must, need to and have to in 2017

2274 and found that the frequency rates remain very similar: have to is still the most

2275 frequent deontic modal, and must and need to are used at a similar rate. I found that

2276 should, which was not considered by Johansson (2010), is used marginally less than

2277 have to in the COCA but more frequently than must and need to. Without knowing

2278 how Johansson's (2010) search was conducted, or how the frequency figures were

2279 treated (rounded up or down) a true comparison cannot be made, however.

2280 Some of the reasons for the increase in use of need to and the fall off of must

2281 may well relate to the use of the need to as a democratic, non-threatening term

2282 (Nokkonen, 2006, p46). Smith (2003) found that need to was used around 130\% more

2283 in written American English over the course of 3 decades (between approximately

2284 1960-1990) and 249\% more in British writing, while in spoken British English its use

2285 increased by more than $600 \%$ in the same period. 'Need to grows in use in all

2286 syntactic environments, and in some of these it is likely to be a competitor with must

2287 and have to' said Smith (2003, p255) who adds that need to 'can acquire the force of

2288 an imposed obligation -something that does not happen with other markers - the writer

2289 or speaker can claim that the recommended action is merely being recommended for

2290 the doer's own sake (2003, p260). Medical advice may well be the kind of

2291 recommended action that Smith has in mind.

2292 Should seems to be consistently frequent in studies of modal verbs (e.g. Collins, 2293 2009). Leech (2004) suggests that should is less categorical than must in both

2294 obligation and logical necessity and this obligation can be reduced to 'something like

2295 desirability'. (p158). Nokkonen (2006) says that should 'gives the impression that the 
2296 speaker is appealing to the assumed needs of the addressee' (p64) which suggests that 2297 any advice or perceived obligation is principally for the good of the recipient. This has 2298 also been said about need to as we have seen and suggests that we may see a high rate 2299 of occurrence of should and need to in patient information. Health advice is generally 2300 offered, after all, for the good of the patient recipient. I expect, too, to see have to used 2301 frequently, based on the findings of studies of modal use reported in this section 2302 (Collins, 2009; Johansson, 2010; Smith, 2003).

2303 At the outset of my doctoral investigations, I was unsure what to expect with 2304 regards to the frequency of must in patient information. Collins (2009) found must to 2305 be used slightly more frequently than need to in GB and Australian English, though 2306 need to was preferred in the American data. The change in frequency of must, 2307 however, is reported as gradual by Johnsson (2010) and the drop off in use is greater 2308 in spoken language, where it retains a strength that many users would find 2309 inappropriate. Patient information is written material produced by a medical authority, 2310 however, and as a result, might be considered fairly formal in style. In spite of the 2311 simplified language and question-answer format that is intended to simulate a 2312 conversation, my analysis of lexical bundles revealed that two-thirds of the 4-word 2313 bundles extracted were of a structural type more often seen in academic prose, with 2314 one-third conversational bundle types. Perhaps must would be retained as the deontic 2315 modal of choice in patient information.

2316 This concludes my presentation and discussion of the frequency of deontic 2317 modals in British, Australian and American English and the changes in use over time, 2318 as reported in the literature. I now turn to the methodology employed in my analysis, 2319 presenting a summary of the key steps. The methodology has been presented in full in 2320 chapter 3 . This is followed by a presentation of the results of the different analyses 2321 undertaken and a discussion of the key findings.

\section{6.6 Methodology}

2323 The methodology of this corpus-based analysis has been presented in detail in chapter

23243 , though in this section I re-present the key steps. I will first present the corpora used 2325 in the analysis. 
2327 The 408, 997-word corpus of patient information was made up 221 downloadable 2328 patient information leaflets, sourced from three principal organisations: the NHS, the 2329 Royal College of Radiographers, and RadiologyInfo.com, a website associated with 2330 the Radiologic Society of North America (RSNA). The corpus was first compiled in 2331 2011, with later additions in 2014 and 2016. Both diagnostic and therapeutic 2332 procedures were included, though the majority of documents relate to diagnostic 2333 exams, and medical procedures involving the use of radiographic technologies.

2334 Two further corpora were also used to provide a comparison of the frequencies 2335 of deontic modals. These are described below.

\section{6.6.2 Comparative corpus 1: consumer advice}

2337 This was a small, 104,670-word corpus of consumer information, with material from 2338 both the UK and the US. The inclusion criteria for the consumer information corpus 2339 was very close to that for the patient information corpus. All texts were available as 2340 Word or pdf documents on the Citizens Advice website, a recognised authority in the 2341 UK for consumer information, and its US equivalent, the Federal Trade Commission 2342 (https://www.ftc.gov/). This was a much smaller corpus than the patient information, 2343 however, with a word count of just 104,670 and the majority of texts came from the

2344 UK Citizens Advice site (https://www.citizensadvice.org.uk). The topics covered 2345 included housing, health, children, consumer topics and the law. The length of the 2346 documents included in this corpus also varied, from the longest at over 7,000 words to 2347 the shortest at under 300 words. The longest documents in this corpus were from the 2348 UK, unlike those in the Patient Information corpus, where we saw that the longest 2349 documents were US-sourced.

2350 By comparing the frequencies of deontic modal verbs in consumer advice with 2351 those found in patient information, I wanted to see if patient information resembled 2352 consumer information, particularly in light of the fact that patients are increasingly 2353 referred to as consumer or clients. The latter is very much focused on consumer rights 2354 in the law. Patient information is, in itself, a right; the right to be informed of 2355 healthcare-related events. The information confers on the patient the ability, in theory, 
2356 to also be involved in decisions relating to healthcare and treatment by virtue of being

2357 informed. And while we are led to believe the customer is always right, a position that

2358 the law can uphold, we never hear the same said about patients. On the basis of this,

2359 my hypothesis was that deontic modal verb use would not be the same, as there are

2360 fundamental differences in the functions of healthcare information and consumer

2361 information. This comparison I hoped would add to my growing understanding of the

2362 lexical characteristics of patient information.

\section{6.6.3 Comparative corpus 2: General radiography}

2364 The 719,209-word corpus of General radiography is made up of a radiographer

2365 handbook, Clark's Positioning in Radiography, a training textbook, Patient Care for

2366 Radiography and research from Radiography, a peer-reviewed journal of the Society

2367 and College of Radiographers and the European Federation of Radiographer Societies.

2368 The research was included because it is written for and by radiographers, and not

2369 radiologists, and thus deals with the issues that are relevant for radiographers: patient

2370 safety, radiation dose, patient position and workflow, for example. Radiologists, on

2371 the other hand, are doctors who specialise in radiology. Their job is to diagnose and

2372 propose treatment. Radiographers, on the other hand, are the healthcare professionals

2373 who carry out radiographic examinations.

2374 By comparing the frequency rates of deontic modals in patient information for

2375 radiography with those found in other radiographic registers - textbooks, manuals, and

2376 research papers - I thought I would be better able to characterise some of the uses of

2377 deontic modal verbs specific to patient information, rather than specific to the field

2378 radiography.

2379 6.6.4 Search criteria

2380 Sketch Engine was the software used in this analysis. The steps taken, when compared

2381 to those of the keyword extraction and the lexical bundle analysis, were

2382 straightforward. In Sketch Engine, I used the so-called Simple search, which is

2383 cleverer than the name implies. The software works out what it is you are looking for

2384 based on the kind of search term you have entered. If you enter a lemma, the search is

2385 a lemma search, meaning go will also find goes, going. If you enter a term which is 
2386 not a lemma, the software will search only for that word. It was the Simple search that

2387 I used for the modal and semi-modals under investigation, and the software treated my

2388 search terms as lemmas. This meant that the Simple search captured the changes for

2389 person and tense that took place with three of the semi-modals (need to, have to and

2390 have got to), as well as including negative forms for all modals and semi-modals. It

2391 did so quickly and effectively.

2392 There were three distinct steps in my analysis which I summarise in the follow 2393 sections.

2394 6.6.5 Methodological steps 1-3

2395 6.6.5.1 Step 1: General frequency rates of modals in patient information

2396 In order to find out how the frequency of modal verbs in general compared with what

2397 we know of their frequency in general English, a summary of which I presented in 6.4,

2398 I carried out a Simple search in the patient information corpus of the following modal

2399 and semi-modal verbs: can, could, will, would, may, might, must, have to, should,

2400 have got to, need to, need, ought to, be allowed and be supposed to. Raw frequency

2401 counts and their adjusted frequencies in per million words were noted.

2402 6.6.5.2 Step 2: Deontic modals frequency rates in all three corpora: patient

2403 information compared with consumer advice and general radiography

2404 The deontic verbs from the list in 6.6.5.1 were searched, using the same Simple search

2405 described above, in all three corpora. Results were compared using raw frequency

2406 counts and adjusted frequency.

2407 6.6.5.3 Step 3: Deontic modals in patient information investigation

2408 Four deontic modals, found to be the most significant in patient information, were 2409 investigated qualitatively in the corpus: have to, must, need to and should. This was

2410 done by investigating a sample of 100 examples of the modal verb in context. This

2411 facility is available in Sketch Engine, with a default setting of 250. I chose 100 to

2412 reduce the amount of data. 
2414 The first analysis was to investigate the frequency and the use of a range of common

2415 modal and semi-modal verbs in patient information, not only deontic modals but

2416 epistemic and dynamic modals. The results are seen in Figure 9 below. The figures are

2417 adjusted to per million words. Adjustment was necessary as the three corpora were of

2418 entirely different sizes.

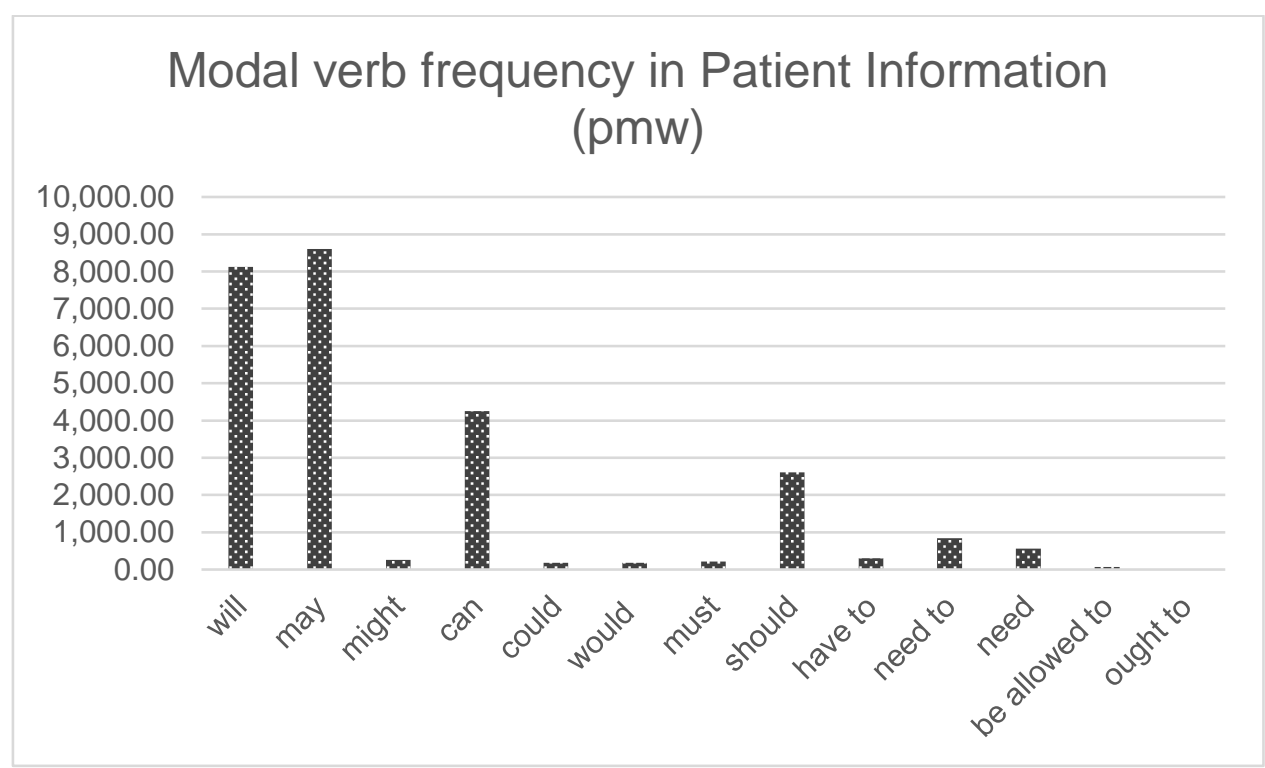

Figure 8 Frequency of common modal and semi-modal verbs in patient information

2421 May, will, can and should are the most frequent modal verbs in patient

2422 information, in descending order of frequency. This is followed by need to and need.

2423 Would and could are very infrequent, as is might and must.

2424 With regard to the second analysis, the frequency of deontic modals in the

2425 three corpora, the corpus of patient information, the corpus of consumer information

2426 and the corpus of general radiography, the results are shown in Figure 10 below. Had

2427 better, ought to and have got to resulted in zero or a single count (ought to in the

2428 corpus of patient information) and thus were not included in the graph. 


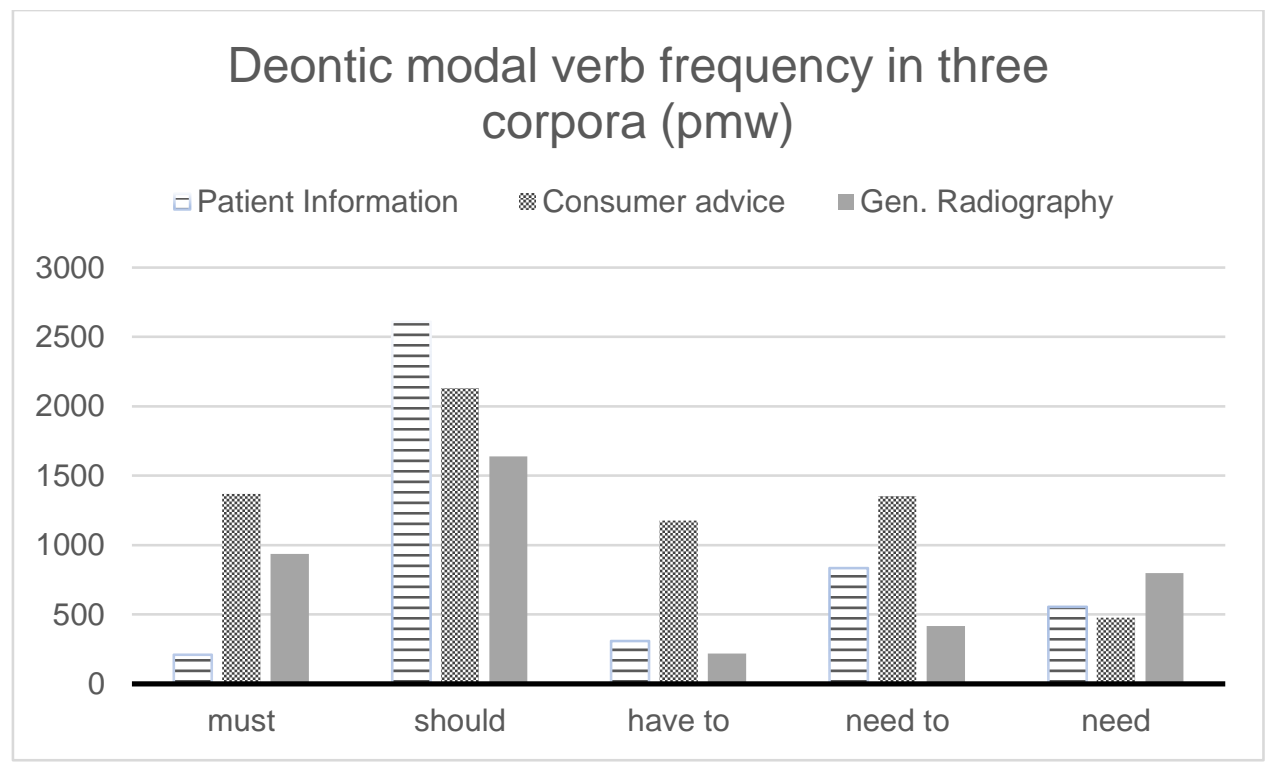

2430 Figure 9 Frequency of deontic modal verbs in the three corpora

2431 The same data is also shown Table 20, to allow for easier comparison, giving

2432 the raw occurrences in brackets following the adjusted frequencies to per million

2433 words. The corpus with the highest frequency of the modal is shaded.

2434 Table 20 Deontic modal verb frequency in the three corpora

\begin{tabular}{llll}
\hline Modal verb & Patient Information & Consumer advice & Gen. Radiography \\
\cline { 2 - 4 } must & $209.87(98)$ & $1368.78(172)$ & $936.99(674)$ \\
should & $2610.56(1219)$ & $2132.76(268)$ & $1639.04(1179)$ \\
have to & $308.38(144)$ & $1177.79(148)$ & $218.87(156)$ \\
need to & $835.21(390)$ & $1352.87(170)$ & $417.06(300)$ \\
\hline need & $556.40(260)$ & $477.22(223)$ & $797.57(574)$ \\
\hline
\end{tabular}




\section{6.8.1 General frequency of modal verbs in patient information}

2438 I will begin this section by discussing the findings of the first analysis, the use and

2439 frequency of common modal verbs, in patient information. This analysis was carried

2440 out to give me an idea of how patient information reflected modal verb use in general

2441 English.

2442 It is immediately obvious from the initial frequency analysis, shown in Figure

24439 , that modal use in patient information differs from the frequencies reported in

2444 studies of general and academic English, where will, would, can and could are the

2445 most frequent modals, in both spoken and written discourse. (Biber et al., 2002;

2446 Kennedy 2002; Leech et al., 2009). In my study of patient information, may is the

2447 most frequent modal, with an adjusted frequency of 8,609 per million. In the Longman

2448 Spoken and Written English (LSWE) corpus, as reported by Biber et al. (2002), may is

2449 used around 1000 times per million, putting the very high frequency of this modal

2450 verb in patient information into perspective. What might be the explanation?

2451 We have seen in our discussion of lexical bundles in chapter 5 that there are a

2452 lot of vague and imprecise references in patient information, particularly related to

2453 time. Medicine itself is sometimes very vague, as so little can be predicted with any

2454 great certainty and may also appears to be very common in some medical writing:

2455 Vihla (1999) found may to be the most common modal in a number of the registers

2456 she examined. We have also seen that the threat of legal action is never too far away

2457 in modern medicine: may confers a legal advantage over the surety offered by will.

2458 Without examining the use of may in detail in patient information, however - and may

2459 is not the focus of my study - we cannot be sure how the modal is being used. It would

2460 undoubtedly make an interesting future study.

2461 The next most frequent modal in patient information is will, close behind at

24628,122 per million. This is also used at a very high rate of frequency in patient

2463 information. Will is the most frequent in general academic English and conversation,

2464 though Biber et al. (2002) report a frequency rate in the LSWE of just over 3,500 per

2465 million, less than half the rate in patient information. May and will together occur 
more frequently than all of the nine modal verbs in the LSWE corpus combined, as

2467 reported by Biber et al. (2002). Looking at my data again, it seems that a few modal

2468 verbs are being used with great frequency (may, will, can, should) and an equal

2469 number used barely at all (might, could, would, must and have to). Between the two

2470 extremes we find need to and need, which are used with reasonable frequency, and to

2471 which I will return later in this discussion section.

2472 Could and might are often used in academic prose and conversation to mark

2473 logical possibility, along with may, but are barely used in patient information. Could

2474 appears at a rate of 182 per million, while might occurs at an adjusted rate of 263 per

2475 million words. Could and might usually express doubt, as does may, though could and

2476 might seem more tentative. (Biber et al., 2002). Perhaps patient information writers,

2477 though happy to use epistemic may with great repetitive frequency, do not wish to

2478 sound overly tentative and thus avoid could and might. An alternative explanation

2479 might be that could and might are victims of the message simplification that patient

2480 information is subject to: after all, why use could and might when you can repeat may?

2481 Message simplification does not seem a likely explanation for the absence of

2482 must and have to in patient information, however. The very low rate of frequency,

2483 particularly of have to, is especially interesting when we remember the reported rates

2484 of have to and must in Collins (2009) and Johansson (2010) reported in 6.5.1 and

2485 6.5.2: have to was by far the most frequent deontic modal in both studies. Why are

2486 these modal verbs not being used in patient information when they are so common in

2487 many other registers, including consumer advice, radiography textbooks and

2488 radiography research? I will return to this question later in this section, as the use - or

2489 not - of deontic verbs in patient information is the central focus of this chapter and

2490 will be explored in more depth.

2491 Returning to the general frequency of modal verbs in patient information, can

2492 is the next most frequent in patient information, but at 4,253 per million, it occurs at

2493 around half the rate of may and will. The fourth most frequent is neither would nor

2494 could, which barely feature in patient information, but should, the first of our deontic

2495 modal verbs. Should is used in patient information at a rate of 2,610 per million. Biber

2496 (2002) finds should as common as must in academic writing, and more common than 
2497 must in conversation: in patient information, however, should occurs more than ten 2498 times as frequently as must, which appears at a rate of 209.8 per million. In Collins's

2499 (2009), the results of which were shown in Figure 8, should appears with about twice

2500 the frequency of must in all three varieties of English studied.

2501 In patient information, modal verb use and frequency patterns do not resemble 2502 those seen in general English. A small number of modal verbs are used with very high 2503 rates of frequency in the register, while could and might along with hypothetical 2504 would, all very common in general academic English and conversation, are used with 2505 very low frequency rates in patient information.

2506 Let us turn now to a discussion of the findings of the second analysis, a 2507 comparison of the frequency of deontic modals in the three corpora built for this 2508 doctoral thesis: patient information, consumer advice and general radiography.

2509 6.8.2 Deontic modals in consumer advice and general radiography

2510 Consumer information differs from patient information in that its primary purpose 2511 seems to be informing consumers of their legal rights. Obligations are most frequently 2512 referred to in consumer advice when they are legal obligations, though even here, the 2513 emphasis is very much on the legal rights - the possibilities within the law - that the 2514 consumer has. The emphasis given over to rights can be seen in the repetition of the 2515 word rights and the categories that greet a visitor to the Citizens Advice UK website ${ }^{6}$, 2516 probably the best-known consumer advice association in the United Kingdom. The 2517 Health section homepage refers to a consumer's rights to healthcare on the NHS; their 2518 rights to dental care when abroad; the rights to healthcare for people resident abroad; 2519 how to report discrimination and how to complain.

2520 We saw that patient information does not use could, might, have to or must at 2521 all frequently. In consumer advice, however, they are all used with more or less with 2522 equal frequency, at around 1300 times per million. Should is used most frequently at 25232132 per million. Need to and must are used with very similar frequencies in

\footnotetext{
${ }^{6}$ https://www.citizensadvice.org.uk/
} 
2524 consumer information, at around 1350 per million, while have to is just a little less

2525 frequent at around 1117 per million. In short, a wide variety of deontic modal verbs is

2526 used in consumer information and used with high rates of frequency.

2527 In consumer advice corpus, more than $90 \%$ of the uses of have to involve the

$25282^{\text {nd }}$ person pronoun. The modal is generally used to refer to obligations, legal or

2529 procedural, or to give what amounts to instructions.

2530 (1) You'll probably have to pay a fee to cancel a contract if you've decided you

2531 don't want it anymore.

2532 (2) You can't get your service provider to chase someone who's not named on the 2533 bill-you'll have to get the money from them yourself.

2534 The frequency of have to in consumer information is more in line with

2535 reported studies of modal verb use in UK and US English, and there is variety shown

2536 that patient information seems to be lacking. I have already referred to the emphasis

2537 on legal rights and obligations in consumer information; must is generally used in the

2538 context of laws, requirements and other enforceable aspects.

2539 (3) To qualify they must meet all of the following criteria:

2540 (4) Landlords must tell you if they will not rent to you because of information in your

2541 credit report or background report.

2542 (5) The law which says you mustn't be discriminated against is called the Equality Act 25432010.

2544 Unlike patient information, one of the primary functions of consumer advice is 2545 to tell consumers about their legal rights. In nearly every case in my corpus, when the 2546 topic is the law, must is the modal verb used. While patient information does concern

2547 itself with the law, it does so in the form of a disclaimer, as a form of protection for 2548 the hospital or medical system against the patient; consumer advice, on the other hand, 2549 transmits information about the legal rights of its readers. This is a significant 2550 difference. 
Before I move to an in-depth discussion of the deontic modals in patient

2552 information, let us see how the frequency of deontic modal verbs in the general

2553 radiography corpus compares. The data is reported above in Table 19.

2554 The first thing we see is that there is no one modal that is used with greater

2555 frequency in this corpus. Should is the most frequent deontic modal, but it occurs less

2556 frequently in this corpus than in the other two. Have to, in complete contrast to its

2557 frequency in consumer advice, is used even less often here than it is in patient

2558 information, at little more than 218 times per million words. This is perhaps less

2559 surprising when we remember that a large part of the general radiography corpus is

2560 made of research papers from the Radiography journal, where the informality of have

2561 to would be inappropriate. Must is four times as frequent here than it is in patient

2562 information, though less frequent than in consumer advice. Inspecting the uses in the

2563 corpus, I found that must in the general radiography corpus is not concerned with legal

2564 rights, as it is consumer advice, and is rarely used to express an obligation; rather, it is

2565 overwhelmingly related to logical necessity, as the following examples show:

2566 (6) The central ray must pass through the joint space at 90 degrees to the

$2567 \quad$ humerus, i.e. the epicondyles should be superimposed

2568 (7) The examination must not proceed unless the radiographer is sure of the

2569 identity of the patient

2570 (8) The patient must be monitored with a pulse oximeter during the procedure,

2571 because it is not possible to monitor the patient directly.

Aside from radiography research, the general radiography corpus is made up

2573 of a radiographer's manual related to patient positioning, and a training textbook, with

2574 a large number of instructions, many of which relate to successful and safe imaging.

2575 They are, as a result, instructions presented as necessities.

Let us now move to a more detailed look at the deontic modal verbs as they appear in patient information for radiography. I will take each modal verb, being with

2578 need to, which is followed by need. I will then discuss the use of must and have to 2579 before turning to a discussion of should, the most frequent deontic modal in my 
2580 corpus of patient information. Before the chapter concludes, I present the findings

2581 from a survey of modal verb use that I carried out in the course of this doctoral

2582 research.

2583 6.9 Deontic modal verbs in patient information

$2584 \quad 6.9 .1$ Need to

2585 Need to is the second-most frequent modal with a deontic meaning in the patient 2586 information corpus, and is used three times as frequently as must and two and a half 2587 times more frequently than have to.

2588 The appearance of need to in my corpus may be explained by the fact that, as 2589 was suggested by Johansson (2010) earlier in this chapter, need to has seen an 2590 enormous rise in use in spoken English over the last few decades. Smith (2003) found 2591 that need to was used around 130\% more in written American English over the course 2592 of 3 decades (between approximately 1960-1990) and 249\% more in British writing, 2593 while in spoken British English its use increased by more than $600 \%$ in the same 2594 period. 'Need to grows in use in all syntactic environments, and in some of these it is 2595 likely to be a competitor with must and have to"' said Smith (2003, p255) who adds 2596 that need to "can acquire the force of an imposed obligation -something that does not 2597 happen with other markers - the writer or speaker can claim that the recommended 2598 action is merely being recommended for the doer's own sake' (2003, p260). Medical 2599 action may well be the kind of recommended action that Smith has in mind.

2600 The most common forms of need to in the literature do not correspond to that 2601 used in patient information, however. Smith (2003, p. 261) reports that $1^{\text {st }}$ person 2602 plural (we) and passivized $3^{\text {rd }}$ person are far and away the most common grammatical 2603 subject. Examining my data, it transpires that in patient information, the $2^{\text {nd }}$ person is

2604 far and away the most common grammatical subject: $75 \%$ of structures involving 2605 subject pronouns used you, which was used exclusively in the singular:

2606 (9) Lose weight if you need to.

2607 (10) You will need to stay in bed for two to four hours. 
2609 The 1st person plural, we, was barely used with this structure and appeared just

2610 five times in the entire corpus. These structures, then, are generally addressing the

2611 patient and, as Smith (2003) suggests, are often imposing an obligation on the patient,

2612 though that obligation is within the context of medicine, and thus for the patient's own 2613 good.

2614 The use of need to structures in the passive were also a feature - around one 2615 quarter were of this kind. either using the semi-modal in the passive or where the

2616 following verb was passivised. Most of these structures involve medical procedures or 2617 treatment,

2618 (12) These injections may need to be given several times a day.

2619 (13) Certain foods and medications may need to be avoided prior to taking the test.

2620 We have seen already that a number of lexical bundles were in the passive 2621 form and I have suggested that the focus on the patient in printed information 2622 sometimes forces a passive when an active structure would be more appropriate. In 2623 (12) and (13) above, there seems no explanation for choosing a passive over an active 2624 sentence so the surprisingly high use of passive structures may be explained by patient 2625 information writers failing to follow guidelines that encourage not to use the passive.

In (12) and (13), and most of the examples of passivised need to, the modal is 2627 not being used as an obligation but to express what Collins refers to as dynamic 2628 necessity (2009) or what Biber et al. (2002) call logical necessity. There is a sense that 2629 the situations referred to are, by being a requirement of an objective medical 2630 procedure, outside of the control of either the patient or the health professional. This 2631 fits with the notion expressed by Collins $(2009, \mathrm{p} 74)$ that $3^{\text {rd }}$ person deontic uses of 2632 need to are very often expressions of institutional requirement.

The idea of institutional requirement is also expressed by the core modal need, 2634 which appears at a surprising rate in patient information, almost three times as often as 2635 must, and twice as often as have to, which is, as we have seen, the most frequent 2636 modal in general spoken English after should. Need was also very frequent in the 
corpus of general Radiography, which is made up of manuals, textbooks and research

2638 paper. The very high rate of use of need in patient information compares to its very

2639 infrequent use in Collins' (2010) analysis, seen in Figure 8 in this chapter. Leech

2640 (2003) and Smith (2003) refer to the huge decline in need, which seems to be matched

2641 by an equally large increase in the use of need to.

2642 Although not strictly a deontic modal as the others in this chapter, need is

2643 relevant to my study as it is used in patient information to present requirements. These

2644 requirements are either personal to the patient, as in (14), a general medical

2645 requirement (15), or a requirement specific to the radiography appointment.

2646 (14) If you need an interpreter or information about your care in a different

$2647 \quad$ language or format, please get in touch

2648 (15) In extremely rare cases, surgery may be needed

2649 (16) The images from the scan will need careful analysis by our staff

2650 Returning to need to for a moment, in terms of its behaviour, need to is not

2651 immune to subjectivity, and it is with the 2nd person singular that we see need to at its

2652 strongest. Collins (2009) says that 'in the contexts where there is an obvious authority

2653 structure, the utterance will have the force of a directive' (p73). The uses of need to in

2654 the patient information corpus are often ambiguous, however, and the vast majority

2655 are related very closely to aspects of the medical procedure to be undertaken, even

2656 when used with a $2^{\text {nd }}$ person pronoun. There are very few that might be interpreted to

2657 be a strong directive.

2658 And while there can be very strong exhortations made with the ' $\mathrm{I} /$ we need you

2659 to...' structure, there were only two examples in the entire corpus, and both found in

2660 the same document (and thus almost certainly written by the same person).

2661 Johansson (2010) found that the 'I/we need you to...' structure, though small in

2662 absolute frequencies, was consistently growing in use and in the COCA corpus was

2663 most common in the Spoken and Fiction genres. There is a strength and non-

2664 compromising authority to this structure (that Yagoda (2006) refers to as the

2665 'kindergarten imperative') that seems very out-of-place in patient information, which, 
as we have seen avoid overt references to obligation and authority. However, as

2667 linguistic innovations are seen first in spoken discourse, perhaps it is only a question

2668 of time before this structure becomes more acceptable (and less face-threatening) in

2669 written discourse.

2670 A number of studies have found that need to tends to be used by those in 2671 positions of authority (Glass, 2015; Nokkenen 2006; 2012), such as teachers talking to 2672 students and bosses to workers. It is, at the same time, very frequent among teenagers 2673 (Nokkonen, 2006, p46) suggesting that it is also considered to be a democratic, non2674 threatening term. Need to is also used to appeal to the needs of the addressee and to 2675 issue an instruction or obligation in an objective, polite way (Smith, 2003).

While its multiple uses might explain its rise in popularity, the question is raised 2677 of how need to can be at the same time authoritative, democratic, directing, and 2678 polite? Glass (2015) believes the relationship between the speaker and addressee is 2679 fundamental to understanding the use and meaning of need to and this relationship (or 2680 perceived relationship) explains why the use of need to is neither monolithic or 2681 consistent across contexts. In some contexts, need to can come across as bossy; in 2682 others, it can appeal to external, objective needs. In (17), these needs relate to 2683 information regarding risks. This sentence has a very bossy, we-know-best-tone about 2684 it, which may be because the subject is risk information. In my data, reference to risk 2685 and complications were often presented in this way, as if the patient was being told to 2686 take their fingers out of their ears. The right to know is at the centre of patient centred 2687 medicine, but the right not to know also exists, and is often an ethical dilemma.

2688 Perhaps it is precisely because of the ethical considerations that I react negatively to 2689 the use of need to in (17).

2690 (17) There are, however, several possible risks and complications. These are very imperative in (19) and reference to future instruction, in (18). There is no doubt that

2694 need to is used to tell the patient what to do in these sentences, but the tone is quite 2695 different from that in (17). These are obligations presented as being for the good of the 2696 patient (Smith, 2003) 
(18) You will be taken to the recovery area where you will need to stay in bed for two to three hours, or as instructed by your nurse. overnight

2702 overtly direct structures (e.g. we need you to), patient information avoids a subjective 2703 or an overly authoritative tone when instructing.

2704 Let us now turn to must and have to, which, as we have seen, were very 2705 infrequent in patient information.

\section{$2706 \quad$ 6.9.2 Must and have to}

2707 Have to, a semi-modal and one that has been reported to have increased in use in both 2708 written and spoken English as must has declined (Collins, 2009, p67), represented less 2709 than $10 \%$ of the deontic modal tokens used. Surprisingly, must was used even less 2710 frequently. These results could well be evidence that in some registers Smith's (2003)

2711 prediction of the increasing status of need to viz have to/must is correct. Need to

2712 appears three times as often as both must and have to in my corpus of patient 2713 information.

2714 Many of the uses of must in the patient information corpora refer to the medical 2715 procedure (as seen with need to), but in addition to patient preparation and

2716 communication, before and after the procedure:

2717 (20) If you have any allergies you must let your doctor know.

2718 (21) If you have any allergies or have previously had a reaction to the dye (contrast 2719 agent), you must tell the radiology staff before you have the test.

2720 In contrast to must, however, have to is not used on any occasion in the patient 2721 information corpus to directly tell the patient what to do. It is not used to issue a 2722 directive or obligation but seems to refer more to procedural necessities - and often to 2723 the possibility of procedural necessities. It seems to be used to mitigate necessity - at 2724 times almost apologetically as we can see in the examples below: 
2725 (22) If you have to undress for the procedure, you will be shown to a private cubicle where you will be asked to put on the gown provided.

(23) If you are given fluid to drink on arrival, you might have to wait an hour before entering the scanning room.

2729 (24) As treatment progresses, you may find you have to pass urine more frequently

2730 (25) It may be possible to perform the scan without you having to change your $2731 \quad$ clothes

2732 (26) We recommend that you do not wear jewellery as we may have to ask you to remove this during your examination

The grammatical flexibility of have to can be seen in the use of other modal

2735 modifiers, usually may and will which are used in around one-third of the occurrences

2736 of have to, though there were very few examples of negatives with must or have to.

2737 Must not was used as an obligation just 6 times in the entire corpus, e.g. you must not

2738 drive, and all examples came from the American material. Do not have to was used

2739 just twice in the British material and 13 times, to refer to the same thing, in the US

2740 materials. In the UK, guidelines (NHS Toolkit, 2003) for the writers of patient

2741 information frequently state that negatives are to be avoided; the data in this thesis

2742 strongly suggests that these guidelines are being followed.

2743 Given the very infrequent use of you + must, (at around $115 \mathrm{pmw}$ compared to

2744 you + should at $798 \mathrm{pmw}$ ), it is evident that this is not a preferred modal for giving

2745 instructions in patient information. Must is used with great frequency in radiography

2746 textbooks and handbooks, however, evidence that it still has a place in certain types of

2747 discourse - the discourse where the relationships between the participants (addresser

2748 and addressee) are not considered equal or where politeness is not something to be

2749 concerned about. In the case of textbooks and handbooks, the relationships are

2750 teacher/learner or expert/trainee and here must is seven times as frequent than it is in

2751 patient information.

2752 If must is rarely used to issues obligations in patient information, have to never 2753 used for obligations, and need to refers primarily to procedural necessities, it appears 
2754 that should is the modal verb relied upon to issues instructions and to refer to

2755 behavioural obligations in patient information.

\section{$2756 \quad 6.9 .3$ Should}

2757 In patient information should appeared 1,219 times, (2,610.56 per million). This is a

2758 frequency rate that is more than six times that of as have to, more than twice that of

2759 need to and a whopping nine times that of must. Its proportional frequency in this

2760 study is similar to the studies that have considered general and academic English

2761 (which find should used as often as must in academic writing and more often than

2762 must in conversations) (Biber 2002).

2763 Leech (2004) suggests that should is less categorical than must in both

2764 obligation and logical necessity and this obligation can be reduced to '... something

2765 like desirability' (p. 158). Nokkonen says that should 'gives the impression that the

2766 speaker is appealing to the assumed needs of the addressee' (2006, p64) which

2767 suggests that any advice or perceived obligation is principally for the good of the

2768 recipient. This has also been said about need to as we have seen earlier in this chapter.

2769 Myhill (1995) considers should to be an individually-oriented modal in contrast

2770 to ought to, which he calls group-oriented, along with deontic must and intentional

2771 will (1996, p339). Have to, in contrast, he refers to as an objective modal and one

2772 which denies any personal involvement; the increasing tendency to avoid overt claims

2773 to authority by the speaker or writer favours should (weak obligation) over must

2774 (strong obligation) and explains the rise in use of should and have to (p 339) - though

2775 in patient information, as we have seen, it is need to, not have to that predominates.

2776 There seem to be few differences in the use of should in British and Australian

2777 varieties of English and it is the deontic use that predominates, though should is less

2778 used in US English (Collins, 2009) in both spoken and written discourse. It is also the

2779 case that the deontic use of should is more frequent in written discourse ( Collins,

2780 2009). I will discuss the meanings of should as they are used in patient information

2781 later in this section. 
In the case of written patient information, the authority is not personal but is the

2783 authority of the medical system, the hospital and the team of professionals. The use of

2784 'we' as a subject pronoun always references this authority in the corpus and is not a

2785 'we' that includes the patient, with a handful of exceptions when 'we' means 'we

2786 humans,' e.g. the air we breathe. However, as discussed in chapter 5, 'we' is

2787 noticeable by its absence in patient information. There are just 326 examples

2788 (698.15 per million) of 'we' in the entire corpus. This compares to 6,544 (14,014.38

2789 per million) for 'you'. Nor are references to the hospital evident: There are 100

2790 (214.16 per million).

2791

My keyword analysis did suggest, however, that the authority being referenced

2792 in patient information are certain medical professionals: the radiologist, the doctor and

2793 the physician. As I discussed in chapter 4, these professionals predominate in patient

2794 information, while radiographer and nurse - the professionals with less status - are far

2795 less frequently referred to. The authority obliging the patient with should in patient

2796 information, then, seems to be the age-old, elevated authority of the doctor, in contrast

2797 to the image of a democratic, modern and equitable health system suggested by the

2798 term patient-centred care.

2799 We have seen that modal verbs possess different meanings and should is no

2800 exception. In fact, should possesses a range of meanings, some of which are very

2801 evident in patient information.

2802 6.9.3.1 Meanings of should in patient information

2803 Should generally possesses two main meanings: one is epistemic in nature and refers

2804 to the likelihood of something happening. The other meaning, the most common, is

2805 deontic, and refers to the 'desirability' of something, though the strength of this

2806 desirability ranges from an obligation, 'do (not do) this please' through to 'it would be

2807 a good idea if'.

2808 To understand how the 1219 examples of should were being used in my data, I 2809 sampled 150. Though not a fine-grained analysis, sampling in this way gives an idea 2810 of proportions, which can be very helpful. The distribution of the meanings of should 2811 in patient information can be seen below in Table 21. 
Uses of should in patient information (1,219/2,610.56 per million)

Epistemic (Likelihood) Deontic (Obligation) Deontic (Desirable)

$31(66.55) \quad 108(231.87) \quad 11(23.54)$

2813 Examples from the data of the different uses can be seen below. Epistemic

2814 uses can be seen in (27) and (28).

2815 (27) This should not last more than a few hours.

2816 (28) It should not be painful and will heal

2817 Negative obligations and obligations can be seen in (29) - (31).

2818 (29) Patients with epidural electrodes should NOT have an MRI.

2819 (30) You should not have a bone scan if you are pregnant or think you might be 2820 pregnant.

2821 (31) You should tell the radiographers if you have ever had an allergic reaction to iodine.

2823 In (32) and (33), should is used with the sense of desirability.

2824 (32) A prevention plan should be discussed with your doctor

2825 (33) Ideally all diabetic patients should be given an early morning appointment

2826 The desirability of the utterance in (33) is made clearer by the adverb ideally, 2827 though (32) contains no such adverb and requires reader interpretation. Reader 2828 interpretation, however, is not always reliable as it requires a certain level of health 2829 literacy, as we have previously seen. What is a prevention plan? Will my doctor 2830 contact me or is it my job? What happens if I don't call my doctor? Will there be a 2831 problem? 
There is an assumption underlying some of these sentences that all languages

2833 use an equivalent modal verb with the same meaning. It is assumed, perhaps, that

2834 speakers of English as a foreign or second language can easily decide which meaning

2835 of should is being expressed. This is a very significant, but under-appreciated problem

2836 with should, which I shall discuss further in 6.9.3.2 below.

2837 In my corpus of patient information, should is used in a variety of grammatical

2838 structures to refer to a wide range of subjects, procedural, pre- and post-procedural,

2839 but also to general medical concerns. This is in contrast to need to, which, as we have

2840 seen, restricts itself to procedural concerns, and is primarily used with a $2^{\text {nd }}$ person

2841 pronoun. Have to is not used to issue any instructions or directives in the corpus, but

2842 seems particularly related to possible occurrences and experiences of the medical

2843 procedure. Must, while used in a variety of contexts, is the least frequent of the four

2844 deontic modals. The utility of should seems to lie in the fact that it has a range of

2845 meanings, and when used to issue a directive it is generally non-threatening and can

2846 reduce the obligation to 'desirability' in some cases. These very characteristics may

2847 also make its interpretation problematic, as I will now discuss.

2848 6.9.4.1 Interpreting should

2849 We have seen in the preceding section that should is used with both epistemic and 2850 deontic uses patient information, and that its deontic meanings lie on a continuum 2851 from suggestion to advice through to an obligation. While my sampling of 150

2852 examples (out of the 1219 that are found in the corpus) of should is not fine-grained, it

2853 suggests that around $70 \%$ of the uses of should are deontic and at the obligations end

2854 of the meaning-continuum. About $20 \%$ are epistemic with the remaining $10 \%$ used to

2855 mean that the action is desirable, but not obligatory. Interpreting which is which

2856 requires, at the very least, familiarity with the subtle pragmatics at play.

A fundamental understanding is that which relates to the medical experience

2858 itself. If a patient has never been to hospital, never had radiography or any other

2859 medical intervention, it becomes less easy to navigate the text: health literacy, as we

2860 have seen, multi-faceted and complex, and a powerful factor in the comprehensibility

2861 of patient information (Zarcadoolas et al., 2005). 
2863 interpreting should more challenging than health literate L1 speakers. Studies show

2864 considerable variation in cross-cultural use and expressions of modality, with non-

2865 native speakers being prone to pragma-linguistic transfer (Hinkel, 1995; Hussin,

2866 2013). As Hinkel (1995, p. 329) says:

Pragmatic and sociocultural assumptions represent fundamental points of reference that may not be frequently questioned by members of a language community in which values and common background beliefs appear to be mutually shared.

2874 a mismatch between meaning and intention may also be relevant to written patient-

2875 provider communication. As the use of deontic modals appears to be culturally

2876 dependent (Hinkel, 1995), it is a reasonable assumption that their interpretation may

2877 also be, and while this seems particularly true for should, it may also be evident with

2878 need to. As I have shown, should and need to are used as important expressions of

2879 obligation and necessity in patient information. This tendency may be potentially

2880 problematic for patients, however. Low health literacy and language and cultural

2881 differences may result in the non-threatening, indirect modals should and need to

2882 being interpreted as suggestions, rather than instructions.

My experience working as an English language teacher to speakers of

2884 European languages suggests that should is usually interpreted to be advice and it is

2885 the weak deontic meaning of desirability that is attached to should, not the strong

2886 deontic meaning of obligation. In Italian, for example, desirability is usually expressed

2887 with the conditional form of the verb dovere (must/have to): dovresti arrivare per le

2888 19:00 (You should arrive for 7pm) This desirability can sometimes be strong,

2889 depending on the context, but in Italian this is not the usual meaning. When students

2890 learn modal verbs in English, the tendency in many classrooms and coursebooks is to

2891 present should only or primarily with its desirability meaning. For students who have

2892 not had the opportunity to study in an English-speaking country (or to have a teacher

2893 who uses corpus linguistics to inform her materials), obligations are expressed with 
2894 must or have to, and advice is given with should. In patient information materials in 2895 English, however, should is used for obligations, and neither must nor have to are used 2896 frequently.

2897 Investigating reader interpretation of modals in healthcare discourse is, I feel, 2898 important research that needs to be carried out. While investigations of that size were 2899 outside the scope of my doctoral study, I decided, nonetheless, to carry out a 2900 preliminary investigation to find out how L1 patient information writers use deontic 2901 modals compared to L2 speakers of English. To that end, I developed a survey which I 2902 describe in the following section.

\section{6.9.4 L1 and L2 uses of deontic modals in healthcare contexts}

2904 In order to investigate whether there are any differences in the way non-native 2905 speakers and native speakers use deontic modal verbs in the context of medical 2906 information, I conducted an online survey with 20 questions, the results of which can 2907 be seen in Appendix C.

2908 Table 22 Survey of deontic modal use in radiography materials: participant info

Survey: use of deontic modal verbs in radiography materials (Dec 2017 - July 2018)

\begin{tabular}{llll}
\hline Speaker-type & N (100) & Healthcare professional? & Medical comm writer? \\
L1 & 64 & 0 & 60 \\
L2 & 36 & 25 & 0 \\
\hline
\end{tabular}

2909106 respondents took part, with 100 finished questionnaires received. 64\% indicated

2910 they were L1 speakers of English. As the respondents were contacted via a British-

2911 based healthcare communication forum, it is likely that the majority were L1 speakers

2912 of British English. Of these, 60 were healthcare communication writers. All but four

2913 of the L2 speakers worked or were training to work, in either radiography or

2914 biomedical science. I cannot know for sure which L2 respondent was working or

2915 training in healthcare as the question did not distinguish. It was also a question I added

2916 to the survey when I realised that this information was important. The first six 
2917 respondents did not answer this question. All L2 respondents had Italian, German,

2918 Hungarian or Romanian as their L1. Four of the respondents were trainers in medical

2919 English with Hungarian and Romanian as their L1s.

2920 Having respondents who were knowledgeable about medicine in general, or 2921 who had knowledge specific to radiography was essential, as we have seen that this

2922 kind of knowledge is needed in order to distinguish a suggestion from an obligation.

2923 To my knowledge, all respondents were either very knowledgeable about radiography

2924 or, as medical communication writers, might be expected to have sufficient

2925 knowledge to appropriately interpret the sentence.

2926 Instructions were provided asking the respondents to select the modal verb 2927 they preferred or considered most appropriate to complete the gap in 20 sentences. 2928 The sentences came from the patient information data in my corpus, so authenticity 2929 was controlled for, and four possible answers were provided. The sentences included 2930 distractors (using non-deontic modal verbs such as can, could, will and might) while 2931 the order of the answers was varied

\section{Results}

\section{$2933 \quad$ L1 and L2 difference}

2934 The results were very surprising. I did not expect to see must used with such

2935 frequency by either group. L1 speakers (over 90\% medical communication writers)

2936 selected must over the other modal verbs. Remember that in my patient information 2937 corpus, must was the least used modal. Should and need to are used by L1 speakers 2938 with very similar frequency in my survey, though in patient information, should was 2939 far and away the most frequent modal, used three times as frequently as need to, the 2940 second most frequent modal.

2941 In the L2 group of respondents, however, there seems to be no preference 2942 whatsoever, with all four modals being used with equal frequency. This was 2943 unexpected, and without further investigation I cannot be sure of the reasons. I have 2944 not yet examined the order in which modals were selected, though it may be that the 2945 modals are being used one after the other, as if they are synonymous, to avoid 
2946 repetition. At the very least, I can say that some differences in use was revealed by 2947 my simple survey, a finding that warrants further investigation.

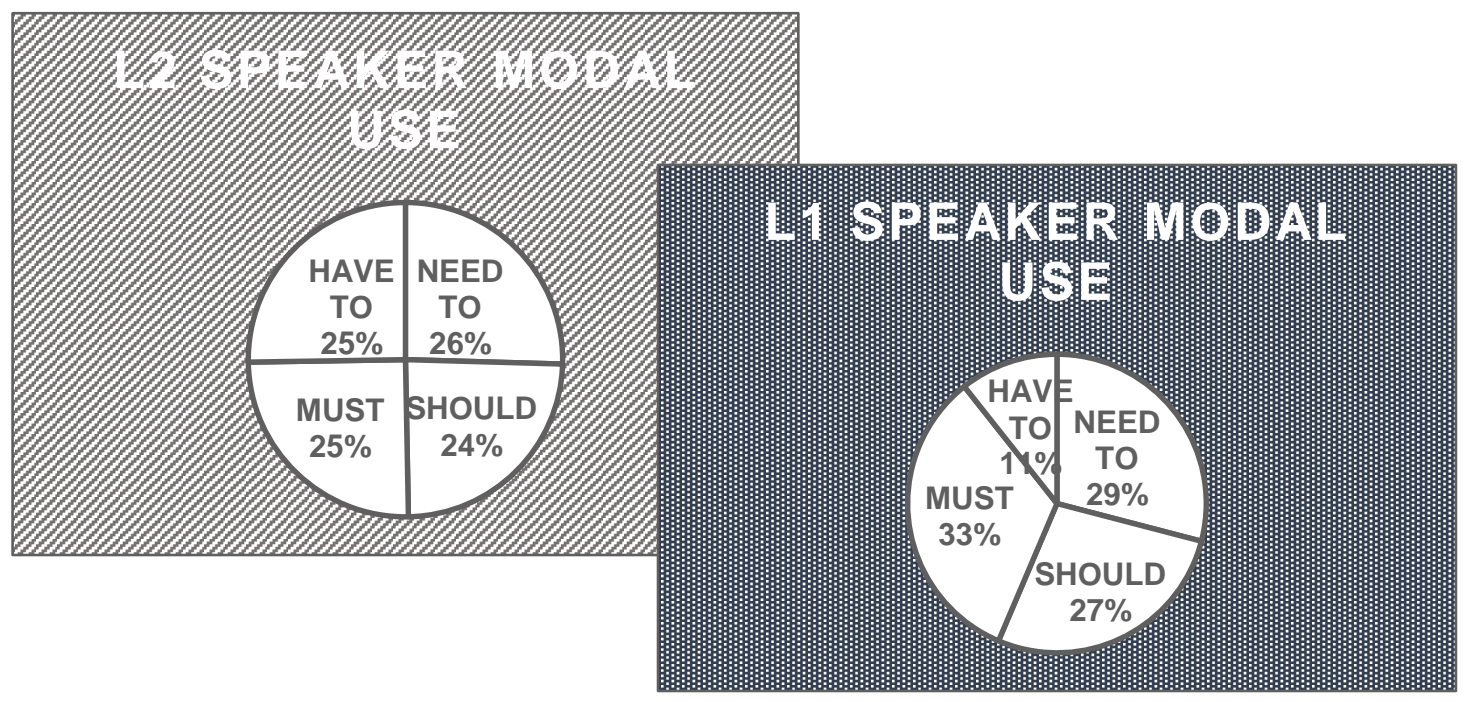

Figure $10 \mathrm{L1}$ and L2 Speaker difference in use of modals

\section{Individual variation}

2953 When I examined the individual responses in the groups, I discovered great variation

2954 though it was particularly marked in the L1 group. Some L1 speakers never used 2955 must; some used it only once, preferring to use should or need to more frequently, 2956 while some respondents chose must most of the time.

2957 This was an exploratory questionnaire that raised more questions than answers, 2958 and while I tried to control for medical knowledge by selecting only qualified and 2959 trainee medical professionals along with practising medical communication writers, it

2960 is possible that some healthcare communication writers and medical English trainers

2961 are not sufficiently knowledgeable about radiography to know if a statement

2962 referenced an action that was desirable or required. This highlights the difficult

2963 position patients may find themselves in when confronted by the same information.

2964 The individual variation that I found was very interesting and could be 2965 explored further by asking respondents to provide their own response, rather than 2966 selecting it from a set of four. My survey asked people to complete a gapped sentence 
2967 for ease and speed. Asking people to read statements using a range of deontic modal 2968 verbs and to rate the degree of obligation would also be a useful study. If knowledge 2969 of the topic could be controlled for, perhaps by using an entirely made-up scenario 2970 that follows recognisably 'sensible' rules, so that only the researchers knew what was 2971 permitted, we might see better some of the mechanisms that lie behind our use of 2972 these modal verbs. I suspect that one reason we do not see many studies of deontic 2973 modals is that capturing users' perceptions of their range of meanings is not at all 2974 straightforward, which, my brief survey suggests is also subject to great individual 2975 variation.

The perception of negative and affirmative statements is also something that

2977 could be explored further. In my questionnaire, two sentences concerned the necessity 2978 to fast (i.e. not eat) before the exam. One sentence used a negative form and the verbs 2979 eat and drink; the second was an affirmative and used the more medical term fast. The 2980 huge individual variation was evident even here: must not was selected by just over $298177 \%$ of the L1 speakers, most of whom were healthcare communication writers. The 2982 second sentence saw just under 30\% selecting must, with the majority (47\%) choosing 2983 need to. Perhaps need to fast sounds better than must fast? A survey that included 2984 interviews to find out what motivates people in their choice of modal verb would be 2985 interesting.

2986 Though a small-scale questionnaire, the appearance of must as the most 2987 preferred modals for L1, and not should, is surprising, and contrasts with the 2988 frequency found in the patient information corpus. In patient information there is a 2989 very clear difference in frequency, with should being used eight times more frequently 2990 than must, and twice as frequently as need to. Further studies are needed to investigate 2991 the reasons healthcare information writers choose one deontic modal over another, and 2992 what meanings are perceived by readers of the materials: the patients. Studies that 2993 investigate the role of language and culture in the perception of obligations and 2994 requirements in healthcare materials are also needed, particularly as it is the 2995 multicultural, multilingual anglophone nations that produce the most patient 2996 information. 
2998 Patient information relies on a high frequency of modal verbs with should and need to 2999 used to express obligations and to instruct the patient. My findings, however, suggest 3000 that while patient information does express obligations, it is keen not to be seen to do 3001 so.

$3002 \quad$ Obligations are phrased politely in patient information, very often using 3003 should, along with $3^{\text {rd }}$ person requirements or obligations (e.g. your health 3004 professional should discuss...diabetics should be given a morning appointment, etc.)

3005 Need to appears with surprising frequency, though in contrast to studies that report $1^{\text {st }}$ 3006 person and $3^{\text {rd }}$ person passivised structures to be the most common in writing, $75 \%$ of 3007 all occurrences of need to in patient information are with the $2^{\text {nd }}$ person. While this 3008 may suggest that it, too, is used to issue obligations and functions similarly to should, 3009 a closer look reveals that the majority of occurrences of need to refer to procedural 3010 necessities. There were no examples of strong obligations being expressed with this 3011 modal. There is a distinction in use, then, that sees should used with a variety of 3012 meanings and referencing different subjects while need to is more restricted.

3013 Interestingly, need to is never used to oblige the patient, unlike should (and far less 3014 frequently, must and have to).

3015 Both should and need to can be used to minimise the voice of authority and to 3016 issue obligations and instructions that are presented as being for the good of the 3017 patient (Smith, 2003, p260). Glass (2015) claims need to does not possess a 3018 monolithic meaning at all, but is dependent on context and relationship between 3019 participants. In patient information, where the patient is increasingly referred to as a 3020 client or service user, and the hospital or professional a 'service provider', the voice of 3021 authority is minimised, and the tone is not overly authoritative. The strong 'we need 3022 you to' structure is never used and need to seems closer to external necessity in this 3023 register.

There is a pattern visible in the use of modal verbs for directives in patient 3025 information: have to, need to and should can all refer to procedural requirements, 3026 though the latter two can also refer to personal or non-procedural requirements. Have 
3027 to is never used in this way. Must is the only modal used for strong obligation but is 3028 also the least used deontic modal in my data.

3029 The use of should and need to as important expressions of obligation and 3030 necessity in patient information may be potentially problematic for patients, however.

3031 Low health literacy and language and cultural differences may result in the non-

3032 threatening, indirect modals should and need to being interpreted as suggestions,

3033 rather than instructions. Culture also influences the roles, and expectations of roles, of 3034 participants in medical interactions and thus how obligations - or suggestions - may be 3035 perceived.

3036 The relationship between the patient and their healthcare professional is far 3037 more ambiguous today than it was a generation ago when it was very likely

3038 considered to be a straightforward non-expert/expert relationship. Patients in the $21^{\text {st }}$

3039 century are increasingly referred to as 'clients' or 'customers', and while the

3040 relationship may not be that of peers, it is increasingly a relationship where the patient

3041 expects to be treated as an equal partner in the healthcare decision-making process.

3042 Written patient information encourages this behaviour, avoiding bald obligation and 3043 encouraging patients to take responsibility for their own healthcare.

3044 Some patients, however, are more familiar with a paternalistic style of 3045 medicine, which is still practised and where the role of doctor still has elevated status. 3046 e.g. in Asia (Clarmita et al., 2011) and former communist countries (Murgic et al., 3047 2015). In cultures that are far less patient-centred, written information is less widely 3048 available and may well be considered irrelevant. Decisions are likely to be made by 3049 the health professional alone. It is reasonable to assume that patients from such 3050 countries will have different expectations of their role, the role of information and 3051 their expectations of the role of the doctor. As a result, the processing of obligations 3052 may be quite different depending on the perception of the role of patient and that of 3053 the doctor. For these patients, bald obligations may not be so face-threatening, while 3054 should may appear as little more than a suggestion. The fact remains, however, that 3055 we know very little about deontic modals in medicine, as they have yet to receive the 3056 same amount of interest as epistemic modals. More studies that look at the use and 3057 interpretation of deontic modals in medical information, both written and spoken, by 
3058 patients with different linguistic backgrounds, could prove very fruitful in the quest to 3059 improve patient-provider communication.

3060

3061

3062

3063

3064

3065

3066

3067

3068

3069

3070

3071

3072

3073

3074

3075

3076

3077 
3079 At the start of this thesis, I set out to describe some of the lexical characteristics of

3080 patient information for radiography by applying three methodological approaches

3081 from the field of corpus linguistics. The motivation for my study lay in the fact that, in

3082 spite of the ubiquity of patient information and its growing importance in many

3083 healthcare systems, the lexical characteristics of the register had not been described.

3084 This absence in the literature also seems particularly hard to explain given the

3085 concerns relating to readability that surround discussions of patient health information

3086 and the growing concerns regarding patients' ignorance of radiography and radiation.

3087 Notwithstanding a handful of studies calling for a new linguistic approach to the

3088 writing and assessment of healthcare messages, lexical descriptions of the register are 3089 absent.

3090 My decision to focus on keywords and lexical bundles was made primarily 3091 because the first two approaches had not yet been used with the register of patient 3092 information. Lexical bundle studies have provided plenty of evidence of the 3093 relationship that exists between the characteristics and communicative purpose of a 3094 register and the structure and distribution of its lexical bundles. The keyword 3095 approach, in turn, has shown itself to be very useful in healthcare communication 3096 studies as it can reveal hidden discourses, underlying beliefs and interesting 3097 communicative patterns that may not be otherwise apparent otherwise. As for deontic 3098 modal verbs, Vihla's (1999) study investigated modal verbs in a wide range of 3099 medical registers but she did not include patient information. My analysis was carried 3100 out on deontic modal verbs to partly fill the gap in the literature, but also because 3101 instructing patients is one of the primary functions of the information produced by 3102 hospital departments. If we understand how obligation and instruction are expressed in 3103 healthcare materials, it may lead to a better understanding of their effectiveness and 3104 why people sometimes do not comply with those requirements. My analyses resulted in a wealth of information regarding the lexical 3106 characteristics of the register with clear implications for the both the development and 3107 evaluation of healthcare materials. My findings also support the notion that standard 3108 readability measures are inappropriate tools to use with healthcare materials. Some of 
3109 the most important findings, and thus the reasons these measures are inappropriate, I

3110 will highlight in this concluding chapter. These findings will be followed by other

3111 aspects of interest revealed by my analyses, aspects which certainly merit further

3112 investigation, though which are outside the scope of the current study. I will also

3113 present some of the limitations to my research.

3114 One of the most significant findings of my research is that, in terms of its

3115 lexico-grammatical characteristics, patient information has more in common with

3116 formal prose than conversation. This may seem a peculiar observation, given that the

3117 patient information that I analysed is written and thus as written discourse it may be

3118 expected to possess lexical qualities appropriate to written prose. However, we should

3119 remember the guides produced for patient information writers that exhort them to

3120 produce information that resembles a conversation (Plain English Campaign, 2001).

3121 At first glance much patient information does seem to be conversational in style, and

3122 many examples of patient information are laid out on the page as if it were a

3123 conversation, with questions as headings. However, closer analysis reveals that the

3124 structure of the text itself is not at all conversational, with two-thirds of the 4-word

3125 lexical bundles used in patient information those that are more commonly found in

3126 formal prose.

3127 The same style guides tell writers to avoid the passive and to use we and you

3128 (NHS Toolkit, 2003), though in spite of the recommendations, the passive does appear

3129 with some regularity in the patient information that I studied. I found the passive used

3130 in $18 \%$ of the 4 -word bundles in patient information, while a quarter of the

3131 occurrences of the modal need to are passive. One reason for the appearance of the

3132 passive, may be that avoiding it entirely is simply impractical advice for patient

3133 information writers. Some information is more naturally presented in the passive

3134 voice and advising writers to use the active voice continually may have an impact on

3135 how the information is read and comprehended. The passive seems more appropriate

3136 given that much of the information in patient information for radiography concerns

3137 what will be done to the patient, why it will be done, how it will be done and how

3138 long it will take. Using the active voice to present this kind of information is not

3139 natural. The use of the passive may explain some of the frequency of the subject

3140 pronoun you which is highly frequent in patient information, appearing at a rate of 
3141 more than 14,000 per million words. We barely features, however, and is used at a rate

3142 of less than 700 times per million words. The focus in patient information seems to be

3143 predominantly on the patient and we may rightly ask whether this is always or entirely

3144 appropriate. Patients need to know who is instructing them, though the voice of

3145 authority (we) is almost entirely absent in the materials I examined. I will return to this

3146 finding later in this concluding chapter.

3147 The characteristics of formal prose are also seen in the use of modals in my

3148 data. May is rare in spoken language and much more common in academic prose

3149 (Biber et al., 2002), though my analyses reveal it to be the most frequent modal in the

3150 corpus of patient information. Have to, on the other hand, the most frequent modal in

3151 conversation (Biber et al., 2002), is barely used in the patient information that I

3152 analysed. An incidental finding that relates to style but also to how conversational

3153 patient information really is, was that there were no contractions used anywhere in the

3154 corpus. Can't appeared as cannot; won't appeared as will not, mustn't appeared as

3155 must not. Contractions, of course, are a feature of spoken discourse. Consumer advice

3156 used contractions frequently, and also came across as the friendlier and less formal of

3157 the two registers. The generally accepted explanation for not using contractions in

3158 writing is that they appear informal, less scientific and/or authoritative, which,

3159 ironically, is precisely the type of writing patient information hopes to be. There does

3160 seem to be some evidence that negative contractions are problematic for readers with

3161 learning disabilities, but I am unaware of evidence that shows that the average reader

3162 has issues with contractions.

3163 The findings relating to the lexico-grammatical characteristics of patient

3164 information can help us answer the research question that relates to the

3165 comprehensibility of patient information for radiography and, by extension, the

3166 appropriacy of readability assessments. As we have seen throughout this thesis,

3167 readability measures generally do not consider the structure of the sentence but

3168 consider the length of the sentence and the length of individual words. If two-thirds of

3169 the 4-word lexical bundles are of a structural type more common to academic prose, it

3170 suggests that in spite of shorter sentences and shorter words, the text may be less

3171 accessible for some patients. Many of these 4-word bundles were referential bundles,

3172 which are also more common in academic prose than conversation. They include 
3173 passive structures such as be used to take and prepositional phrase structures, which

3174 predominate, such as at the end of. These types of bundle are far more frequent in

3175 more formal, information-dense text.

At the very least, patient information texts may have an academic, official

3177 voice, in spite of the simplified text, that may affect how these healthcare materials are

3178 perceived by readers. We should keep in mind that wanting to read information can be

3179 as important as being able to read it: to be effective, a patient information leaflet and

3180 healthcare materials in general must be 'noticed, read, understood, believed and

3181 remembered' (Protheroe, Estacio, Saidy-Kahn, 2015, p. 192, citing Ley, 1982).

3182 Perhaps the inclusion of these structures is not surprising, however, when we

3183 consider the focus in procedural information on time and place, resulting in many

3184 references to time and place using phrasal structures. E.g. after a couple of hours;

3185 within a few days; at the top of. These types of structures are a principle characteristic

3186 of informational discourse, and one of the primary functions of patient information is

3187 to inform. The high-density of information in these healthcare materials suggests that

3188 certain structures - particularly the time and place structures - are inevitable. If that is

3189 the case, more research is needed on how these structures can remain without

3190 rendering the text too difficult to process or too formal in tone. One approach could be

3191 to time patients reading text with a low and high density of referential bundles to see if

3192 processing time is affected; message comprehension and retention can then be tested.

3193 Another significant finding relating to the readability of patient information is

3194 the lack of discourse organising bundles that it appears to use. Discourse organising

3195 bundles function to organise the text by providing signposting and to support the

3196 reader (or listener). They play a significant role in the cohesion of a text, and

3197 cohesion, as we have seen, has a fundamental role in making text comprehensible.

3198 Discourse bundles also appears to have some relationship to how persuasive a text

3199 appears, as we saw in chapter 2, and how well the message of the text is remembered

3200 which is of great relevance to the topic of patient information. In spite of their

3201 function, which one would assume would be welcome in information-dense text, this

3202 category of bundle was the least used overall in the patient information, representing

3203 just $12 \%$ of the 4 -word bundles. While rare overall, however, three discourse bundles 
3204 were seen to be repeatedly used, and were among the first 5 most-frequent bundles in

3205 the corpus. Closer inspection revealed that these bundles were, in fact, framing

3206 instructions, a use which seems to highlight the fact that instruction is the principal

3207 function of patient information.

3208 The relationship of discourse organising bundles specifically and lexical 3209 bundles more generally to the cohesion of a text warrants further investigation and

3210 may certainly have a bearing on its readability. Standard readability assessments,

3211 however, do not measure cohesion nor do they consider the type of lexical structure

3212 used (or the ratio of noun phrases to verb clauses, which in patient information are

3213 2:1). Perhaps the lack of discourse organising bundles and the high number of

3214 referential/academic bundles confound the average patient. Perhaps they have no

3215 effect on comprehensibility, but until studies are carried out, we cannot say for sure. A

3216 similar experiment to that undertaken by Martinez (2002), on the effect of rhetorical

3217 structure on the comprehension and recall of unfamiliar text by ESP students, could be

3218 carried out on a group of patients, controlling for L1 and L2 speakers of English and

3219 type of patient information. An important finding from Martinez (2002) is that the use

3220 of rhetorical structure had a positive effect on the reader's ability to comprehend and

3221 reproduce the information only when that structure was also recognised by the

3222 readers. When they failed to recognise the rhetorical structure in the text, the students

3223 in her study were still able to reproduce the information without having fully

3224 comprehended it What patients are able to recognise as rhetorical structure in

3225 healthcare materials we know nothing about, and while the headings-as-questions

3226 device many healthcare materials make use of is likely to be very helpful for patients

3227 navigating a leaflet, there seems little attention paid to cohesion within the text itself.

3228 It should be remembered, too, that my corpus was made up of patient

3229 information for radiography. Different kinds of patient information exist, of course,

3230 some of it procedural such as the kind investigated here; some of it related to

3231 accessing services in the health system (a particularly ubiquitous kind of information

3232 in the NHS); some of it about specific health conditions; some of it is about medical

3233 devices and equipment that patients need to use. There have been no studies that I am

3234 aware of that investigate how the lexico-grammatical structure of these kinds of

3235 healthcare materials differ from each other, though there is evidence that the 
complexity of the texts differ, when standard readability measures are applied: Protheroe et al. (2005) found that readability levels of various kinds of materials (all found in medical centres and clinics in one region of the UK) differed, with some texts being judged more complex to read than others.

Another reason that standard readability measures are inappropriate (though certainly not the only reason) we first met in chapter 1: readability measures are not a reliable measure of lexical complexity. While my investigations did not concern the characteristics of the content words used in patient information, it is worth revisiting the topic briefly here. The length of a word is not always an indicator of its complexity and this seems particularly the case when we are considering shorter words. If we consider vocabulary used to talk about and to do medicine, we find medical vocabulary on one end of the spectrum, along with nomenclature and highly technical medical terms with Latin and Greek roots. General terms will be found at the other end of the spectrum while influenza might be found towards the middle. Influenza, a medical term, is often abbreviated to flu by lay people and understandable by the majority, if not all, patients. It is also probably close to the middle of the spectrum where we find the words that both patients and providers use, though used with different meanings or connotations. Chronic is a much-used example, meaning 'long-standing and persistent' by the medical professional and 'severe' in some varieties of English. All too often the misunderstanding goes undiscovered as both meanings relate to something unpleasant in need of a solution. Readability measures are likely to class influenza as a difficult word; chronic as an easier word, but neither judgement gives us the whole picture of how patients and providers are using and understanding these words.

As I show in this thesis, however, the complexity of medical vocabulary is just part of the story. When we consider the readability and the effectiveness of patient healthcare materials, the kinds of structure used in the text is also relevant. Readability measures do not distinguish between structural types, such as a verb clause in a longer sentence and a short noun phrase in a short, passive structure. Verb clauses are more common in spoken language, noun phrases more common in more formal written language, though standard readability measures will quite possibly judge the former to be more complex on the basis of length, when in fact it is the latter that is likely to be 
more complex to process. However conversational and accessible a leaflet may

3269 appear, with its cheery colours, large font and chatty headings, if the text itself has a

3270 structure that is more redolent of academic prose, it surely has a bearing on how the

3271 text will be received by the reader and understood.

3272 Assessing the impact of certain structures in the context of healthcare materials

3273 is, I believe, a necessary next step and one which necessitates the involvement of the

3274 patient from the very beginning of the process. As we saw in chapter 2, there have

3275 been repeated calls for patients to be more involved in the production of healthcare

3276 materials, and not simply brought in when assessing the readability of the finished

3277 product. How patients respond to different types of structure, complex and simple, can

3278 be tested, as can the effect of certain types of structure on the cohesion of a text.

3279 Cohesion needs to take centre stage in the production and evaluation of healthcare

3280 materials and care taken that over-simplification does not result in a text that is

3281 stripped of the aspects that make it easier for a reader to process.

This brings us on to the role of health literacy in rendering standard readability

3283 measures inappropriate. Health literacy is, as we have seen (Zarcadoolas, 2011) a

3284 complex, multi-faced skill that goes far beyond the ability to read. The finding from

3285 the modal verb analysis that patient information relies heavily on should (and to a

3286 lesser extent need to) to instruct and oblige patients is an important one that becomes

3287 even more significant when viewed in the context of health literacy. Both of these

3288 modal verbs have been described as appealing to the needs of the patient, thereby

3289 avoiding any overt instructions, so perhaps they are seen as fulfilling the need to be

3290 patient-centred, to be friendly and non-alarming. Should is particularly problematic,

3291 however, as we have seen, as its meanings lie on a continuum with obligation at one

3292 end and mere suggestion at the other. There seems to be a vast amount of information

3293 to be dealt with a priori if the reader is to be fully confident of their interpretation of

3294 should when reading the patient information in my corpus.

As an example, taken from the context of radiography, a patient may well wonder what relevance summer fruits have, or a plate of clams, to a radiographic

3297 exam. When a patient who is booked in for an MRI scan reads that they must inform

3298 staff if they have ever experienced reactions to strawberries or shellfish, does it matter 
3299 if you should tell us or you must tell us is used? The response to this question may be

3300 that it depends very much on whether the patient understand the reasons for asking.

3301 Knowing why the question is important will help the patient to decide whether should

3302 is to be taken as advice, as a suggestion or an instruction. As it happens, people who

3303 experience allergic reactions to shellfish and strawberries will likely experience the

3304 same reaction to the gadolinium contrast fluid that is used during an MRI. It will not

3305 kill the patient, but the reaction is unpleasant and if it can be avoided, so much better

3306 for the patient. When medical professionals use should in this context, they want their

3307 statement to be interpreted as an instruction, not as a suggestion.

3308 We have seen that not only do patients frequently not know which exams use

3309 radiation, they also have a very poor understanding of the concomitant risks of

3310 radiation, suggesting that much background knowledge necessary to interpret certain

3311 types of instruction is lacking. Poor health literacy may mean that if the reason for the

3312 instruction is not understood by all patients it may not be responded to truthfully or

3313 accurately, if it is responded to at all. Our understanding of non-compliance (or non-

3314 adherence, depending on your choice of term) in healthcare will also benefit from

3315 better understanding the meanings that people attach to different modal verbs. 'I

3316 didn't know I had to' and not 'I didn't want to' may turn out to be the real reason why

3317 a patient did not follow what a healthcare professional considered were clear

3318 instructions.

It has also been suggested that patients who are more familiar with a

3320 paternalistic style of medicine may expect clearer instructions, and perhaps not only

3321 these people: older patients living in healthcare systems that pursue patient-centred

3322 medicine may also expect to receive instructions from their healthcare provider in the

3323 clearest, unambiguous way possible. Subtle, indirect structures for some people may

3324 be interpreted to be mere suggestions. There has been no research, to my knowledge,

3325 on the use of these words in healthcare materials, but my findings make it clear that

3326 further investigations are needed.

Language background may well be a factor in the interpretation of modal

3328 verbs, as my simple survey suggested. This is also an area that warrants further

3329 research, particularly as so much medicine is practised in a multicultural environment, 
3330 not only in the UK but in many other countries. This aspect is also relevant in non-

3331 Anglophone societies of course, as English is the global lingua franca of medicine.

3332 The different meanings L2 speakers of English attach to have to, must, should and

3333 need to in the context of medicine also need to be examined. My survey results

3334 suggested there was little distinction, and all were used more or less with equal

3335 frequency. Does that also suggest that many L2 speakers of English understand these

3336 words in a similar way? Or, as many of the respondents were medical students, did

3337 they react as if they were being tested and so hedged their bets by using all the modal

3338 verbs? We need to find out. If the former, the implications are that some non-native

3339 speakers of English may not appreciate that modal verbs of obligation are not always

3340 - or even ever - fully interchangeable. There seem to be very few studies that have

3341 looked at the interpretation of deontic modal verbs and the perception of obligation in

3342 different registers, none at all in the context of healthcare materials. The interpretation

3343 of obligation becomes even more complex when we consider the absence of named

3344 authority in patient information that my research also revealed. The 'blurring of

3345 shouldness' suggests that contextual cues - the knowledge the reader brings to the text

3346 and to surrounding lexico-grammatical structure - take on a vital role in the

3347 interpretation of the degree of obligation. As we have seen, however, health literacy

3348 problems and certain structural characteristics of patient information may mean this

3349 support is absent.

3350 And the story does not end there: my survey also revealed great individual

3351 variety in the use of deontic modal verbs by L1 speakers which was an entirely

3352 unexpected finding and one that most certainly warrants further investigation. This

3353 may suggest that we can never be too sure what a deontic modal is intended to mean

3354 without sufficient supporting context, which may cause few comprehension problems

3355 in familiar contexts but when the context is unfamiliar, being confident of the

3356 interpretation of a deontic modal will be more of a challenge. Health literacy issues

3357 can result in some people misinterpreting the significance of a deontic modal verb

3358 precisely because they lack the contextual cues needed to interpret the utterance.

3359 Contextual cues may also be absent because of the amount of text simplification that

3360 is common in standard healthcare materials. 
My investigations also revealed other interesting findings to complement those

3362 described above. These findings do not relate directly to readability and my data did

3363 not allow any more than a cursory investigation, but I believe they are important

3364 enough to warrant further investigation.

3365 One such finding related to the naming and referencing of healthcare

3366 professionals in the materials. There were many technical and medical terms in my

3367 keyword lists, underlining the complex nature of radiography, though the keywords

3368 that related to professionals in the lists suggested that certain internal inequalities and

3369 bias in medicine are mirrored in patient communication via the naming habits of

3370 medical professionals. The minimal use of the name of the profession that is most

3371 involved with the radiographic examinations, the radiographer and technologist,

3372 alongside the minimal use of nurse is hard to explain, particularly as the role of the

3373 doctor is so marked. Radiologist, doctor and physician are used considerably more

3374 frequently than radiographer or nurse. Why this may be so relates to the second

3375 significant finding I believe, which concerns the impact of gender on information

3376 seeking and information preference.

3377 If it is the case, as reported by Seale et al (2006) and Seale and Charteris-Black

3378 (2008), that a patient's biological sex and/or gender may affect what kind of medical

3379 information they want to read, and if it is the case that older men in particular like

3380 naming experts and specialists and want to read about treatment and therapy and the

3381 details relating to procedures, then it seems that patient information for radiography,

3382 as it currently stands, is more appropriate to the needs of older men. Though tailored

3383 healthcare information is increasingly seen as an effective response to the very

3384 personal nature of the experience of (ill) health, it is only in the last few years that

3385 some healthcare materials have been tailored for sex and gender, and only in the last

3386 few years that public health policies have included sex and gender as a determinant of

3387 health. To my knowledge, the appropriacy of the information presented in procedural

3388 information has been assumed rather than investigated. We have assumed that

3389 radiography information needs to be the same for everybody, without stopping to

3390 think that different people may want quite different types of information. The few

3391 studies where patients were asked their views on leaflets for depression and

3392 rheumatoid arthritis, referred to in chapter 2, found a number of shortcomings in the 
content of the leaflets. Patients reported not finding enough information on certain

3394 topics, which were dealt with in a fairly superficial manner, while some aspects of

3395 living with depression or a chronic condition were never referred to at all. Though

3396 there are only a handful of studies like this, there is a growing awareness among

3397 professional patient information writers and communication agencies of the need to

3398 involve patients and advocacy groups in the developmental process.

3399 Being more aware of the need to involve patients in the development of

3400 healthcare materials does not mean, however, that writers fully understand the impact

3401 of diversity on the content of that information. Where sex and gender are concerned, I

3402 discovered that some writers of patient communication are reluctant to consider sex

3403 and gender as factors that may affect what people want to know. The healthcare

3404 materials writers I contacted seemed entirely unaware of the growing literature that

3405 exists on the influence of sex on many serious health conditions, affecting signs and

3406 symptoms, diagnosis, treatment and outcomes. This lack of knowledge clearly

3407 restricts the approach to the development of patient information. This could prove to

3408 be a very exciting area for further research. Writing information should be a process

3409 that begins with the patient, rather than including them at the end of the process for

3410 their feedback, which often happens. Studies that look at the impact on the quality and

3411 content of patient information materials when patients have been involved at different

3412 stages would also be interesting. Controlling for age, sex and gender, as well as

3413 cultural background, could prove very fruitful.

Methodologically, there are some limitations to my study. With hindsight,

3415 while I wanted to capture some of the accessibility the internet brings in the

3416 development of my corpus, the lexical variation between the US and UK material that

3417 became evident in the results of the key word analysis, suggests that separating these

3418 corpora would have been methodologically more sensible. Not doing so meant that

3419 time was spent double-checking the source of the data while two of the categories of

3420 keywords - legal and treatment - related primarily to US material. There is also the

3421 question of the length of the texts that were selected on the basis of their content. Text

3422 length varied considerably, and the US-sourced material contained many more long

3423 examples than the UK material. The different sizes of the corpora may have resulted 
3424 in different frequency counts for certain items, though I was attentive to any unusual

3425 clustering of the data and make mention in the text where relevant.

3426 Using material from the UK and the US caused some problems, but at the 3427 same time, only using material from these countries is a limitation. I had intended to 3428 look at patient information from a number of different English-speaking countries. I

3429 had assumed that patient information leaflets for radiography would be readily

3430 available on websites, as they were in the UK and the US. This was not the case, and I

3431 found it difficult to find the equivalent in Australian, New Zealand, Canada and even

3432 Ireland. Five years have passed since I first started to gather material, and it is very

3433 likely that patient information is more readily available from these countries. The

3434 question of whether I would have been able to assess the quality of the information, if

3435 it had not come from a medical authority such as the NHS, is also pertinent. I needed

3436 to know that the information I analysed came from a reputable source and was an

3437 example of the kind of information patients would be expected to read. I can

3438 guarantee that applies to the material that makes up my corpus.

3439 Another limitation relates to the adjusted frequency. My decision to use

3440 adjusted frequencies of per million was taken at the beginning of my research and was

3441 not remarked upon until a few weeks before submission. It was suggested to me, at

3442 this point, that an adjusted frequency of 1000 may have been more appropriate, given

3443 the size of my texts and my corpus. I sought further advice and it appears that while

3444 there is some debate among corpus linguists on this topic, many are of the opinion that

3445 small corpora necessitate small adjusted frequencies. However, I feel that an adjusted

3446 frequency of per million has little bearing on my findings, given the types of analyses

3447 that were performed, though on the few occasions where frequency results are

3448 reported, my data will be slightly inflated as a result.

3449 To conclude, I have demonstrated in this thesis that approaching healthcare

3450 information from a linguistic perspective can yield many important insights that both

3451 support findings from the literature and introduce new avenues worthy of exploration.

3452 My research shows that there are characteristics that relate to the lexico-grammatical

3453 make-up of healthcare materials that are potentially every bit as important in the

3454 making-of-meaning for a reader as vocabulary is. The structure of a text can be subtle, 
3455 and while simplifying is the aim of medical communication writers, too many

3456 untested assumptions have been made about what makes a healthcare text difficult to

3457 read: indeed, what many writers understand by 'text simplification' looks overly-

3458 simplified in the light of my findings, many of which relate to the importance and role

3459 of cohesion in reading. Along with tools specifically designed to measure the semantic

3460 complexity of healthcare materials, we need to be able to better evaluate cohesion, and

3461 also to better understand the impact of linguistic features that can contribute to

3462 cohesion, such as lexical bundles. When developing materials, writers need to keep

3463 health literacy uppermost in their minds, at least as far it may affect reader

3464 understanding of the specific health information leaflet in their hand. We also need to

3465 better understand the impact of 'the blurring of shouldness' in healthcare materials, as

3466 we may well find that an absence of authority is not only inappropriate but also

3467 ineffective in the context of healthcare information.

3468 As there is every indication that radiographic examinations such as CT will

3469 become ever more frequent, it is vital that we understand how to develop information

3470 that patients will read, understand and act upon. As the availability and importance of

3471 healthcare materials in general, both digital and print, continues to increase, the

3472 quality of the message and the reader's experience are of paramount importance. 


\section{Appendices}

\section{Appendix A}

\section{LEXICAL BUNDLES EXTRACTED AND CLEANED}

\section{BUNDLE}

1. you may be asked

2. if you have any

3. you will be asked

4. if there is any

5. how do I get

6. It is important that

7. a small amount of

8. may be used to

9. a starting point for

10. will be able to

11. of the inside of

12. if you have a

13. you may be given

14. the best way to

15. you will need to

16. you will feel a

\section{STRUCTURAL}

TYPE
STANCE INTENTION /PREDICTION

DISCOURSE ORGANISERS /TOPIC INTRODUCTION?

STANCE INTENTION / PREDICTION

DISCOURSE ORGANISING TOPIC INTRODUCTION

DISCOURSE ORGANISER/TOPIC ELABORATION

STANCE OBLIGATION/DIRECTIVE

REFERENTIAL TIME /QUANTITY

STANCE ABILITY

REFERENTIAL / INT FRAMING

STANCE ABILITY

REFERENTIAL / TIME/PLACE/TEXT REFERENCE

DISCOURSE ORGANISER / TOPIC FOCUS INTRODUCTION

STANCE INTENTION /PREDICTION

REFERENTIAL /SPECIFICATION OF ATTRIBUTES / INTANGIBLE FRAMING

STANCE/OBLIGATION/DIRECTIVE

STANCE INTENTION /PREDICTION 
17. may also be necessary

18. may need to be

19. You may also be

20. at the same time

21. Are you required to

22. at the end of

23. Who will you see

24. If you have not

25. which is going to

26. a slight chance of

27. to the area of

28. over a period of

29. with the use of

30. so as not to

31. If you would like

32. may be necessary to

33. for a few hours

34. it is safe to

35. the area of your

36. to the site of

37. you may need to

38. you will not feel
STANCE INTENTION / PREDICTION

STANCE/INTENTION /PREDICTION

STANCE INTENTION /PREDICTION

REFERENTIAL / TIME/PLACE/TEXT

REFERENCE

STANCE OBLIGATION/DIRECTIVE

REFERENTIAL TIME/PLACE

STANCE INTENTION /PREDICTION

DISCOURSE ORGANISER? TOPIC

ELABORATION

STANCE INTENTION /PREDICTION

STANCE EPISTEMIC

REFERENTIAL / TIME/PLACE/TEXT REFERENCE

REFERENTIAL /TIME /PLACE/ TEXT REFERENCE

STANCE ABILITY

DISCOURSE ORGANISERS /TOPIC ELABORATION

STANCE DESIRE

STANCE INTENTION /PREDICTION

REFERENTIAL /TIME /PLACE/ TEXT REFERENCE

STANCE /EPISTEMIC?

REFERENTIAL /TIME /PLACE/ TEXT REFERENCE

REFERENTIAL / TIME/PLACE/TEXT REFERENCE

STANCE INTENTION /PREDICTION

STANCE INTENTION /PREDICTION 
39. there is a risk

40. over the area of

41. it is possible to

42. can be used to

43. any of the following

44. that you are taking

45. will make you feel

46. it may be necessary

47. at the site of

48. in the centre/center of

49. are a form of

50. there may be some

51. that is used to

52. for a few seconds

53. to be less than

54. at the time of

55. may advise you to

56. With the ability to

57. on the day of

58. may or may not

59. for four hours before
REFERENTIAL IDENTIFICATION/FOCUS

REFERENTIAL /TIME /PLACE/ TEXT

REFERENCE

STANCE EPISTEMIC

STANCE/ ABILITY

REFERENTIAL /

IDENTIFICATION/FOCUS

REFERENTIAL /IDENTIFICATION/FOCUS

STANCE INTENTION /PREDICTION

STANCE OBLIGATION/DIRECTIVE

REFERENTIAL /TIME /PLACE/ TEXT

REFERENCE

REFERENTIAL /TIME /PLACE/ TEXT

REFERENCE

REFERENTIAL/IDENTIFICATION/FOCUS

STANCE INTENTION /PREDICTION

STANCE ABILITY

REFERENTIAL /TIME /PLACE/ TEXT REFERENCE

REFERENTIAL SPECIFICATION OF ATTRIBUTES / QUANTITY

REFERENTIAL /TIME /PLACE/ TEXT REFERENCE

STANCE INTENTION /PREDICTION

STANCE ABILITY

REFERENTIAL /TIME /PLACE/ TEXT REFERENCE

STANCE INTENTION /PREDICTION

REFERENTIAL /TIME /PLACE/ TEXT REFERENCE 
60. from the area of

61. period of time before

62. sometimes referred to as

63. that may be used

64. that is located in

65. as soon as you

66. you will also have

67. Why do you need

68. can also be used

69. at the part of

70. a specified period of

71. may be used during

72. have previously had a

73. is also used to

74. for a few days

75. to make sure that

76. how deeply you are

77. will be moved into

78. may take up to

79. may be connected to

80. will be placed into

81. as a result of
REFERENTIAL /TIME /PLACE/ TEXT

REFERENCE

REFERENTIAL /TIME /PLACE/ TEXT

REFERENCE

REFERENTIAL/IDENTIFICATION/FOCUS

STANCE ABILITY

REFERENTIAL /TANGIBLE FRAMING

REFERENTIAL /TIME /PLACE/ TEXT

REFERENCE

STANCE/INTENTION PREDICTION

DISCOURSE ORGANISER TOPIC

INTRODUCTION /FOCUS

STANCE ABILITY

REFERENTIAL/TIME/PLACE/TEXT

REFERENTIAL /TIME /PLACE/ TEXT

REFERENCE

STANCE ABILITY

DISCOURSE ORGANISER -TOPIC INTRODUCTION FOCUS

STANCE ABILITY

REFERENTIAL /TIME /PLACE/ TEXT REFERENCE

DISCOURSE ORGANISER - TOPIC

ELABORATION/CLARIFICATION

REFERENTIAL INTRANGIBLE FRAMING

STANCE INTENTION/PREDICTION

STANCE INTENTION /PREDICTION

STANCE INTENTION /PREDICTION

STANCE INTENTION /PREDICTION

REFERENTIAL SPECIFICATION OF ATTRIBUTES / INTANGIBLE FRAMING 
82. in charge of your

83. may be able to

84. we may have to

85. or for people with

86. you may feel a

87. in the form of

88. may also be used

89. If you do not

90. one or more of

91. does not have to

92. If you need any

93. in the treatment area

94. can be treated with

95. at high risk for

96. When will you get

97. please let us know

98. will be shown where

99. will also check the

100. plenty of time to

101. we will ask you

102. the inner lining of

103. that need to be
REFERENTIAL /SPECIFICATION OF

ATTRIBUTES / INTANGIBLE FRAMING

STANCE ABILITY

STANCE OBLIGATION/DIRECTIVE

REFERENTIAL/IDENTITY/FOCUS

STANCE INTENTION /PREDICTION

REFERENTIAL /SPECIFICATION OF ATTRIBUTES /TANGIBLE FRAMING

STANCE ABILITY

DISCOURSE ORGANISER TOPIC ELABORATION /CLARIFICATION

REFERENTIAL /SPECIFICATION OF ATTRIBUTES / QUANTITY

STANCE OBLIGATION/DIRECTIVE

STANCE DESIRE?

REFERENTIAL / TIME/PLACE/TEXT REFERENCE

STANCE ABILITY

STANCE EPISTEMIC

DISCOURSE TOPIC INTRODUCTION/FOCUS,

STANCE/OBLIGATION

STANCE INTENTION /PREDICTION

STANCE INTENTION /PREDICTION

REFERENTIAL /SPECIFICATION / QUANTITY

STANCE INTENTION/PREDICTION

REFERENTIAL /TIME /PLACE/ TEXT REFERENCE

STANCE OBLIGATION/DIRECTIVE 
104. Will try to keep

105. if you need to

106. so that it can

107. will be taken into

108. is a type of

109. In the case of
STANCE INTENTION /PREDICTION

STANCE OBLIGATION/DIRECTIVE

DISCOURSE ORGANISER TOPIC

ELABORATION

STANCE INTENTION /PREDICTION

DISCOURSE ORGANISER - TOPIC

ELABORATION/CLARIFICATION

REFERENTIAL/SPECIFICATION/

INTANGIBLE

\section{Appendix B}

\section{Survey of modal verb preference}

3487 The following sentences come from patient information leaflets for radiography.

3488 Each sentence has a word missing. Select the word you prefer to fill the gap.

3489 There is no right or wrong answer. Simply choose the word you prefer or that you

3490 think is the most appropriate choice.

3491

3492 Is English your first or primary language? *

Yes or no

3493 Are you a language teacher?

Yes or no

3494 Do you work in healthcare or medicine? (including healthcare communications but

3495 NOT as a language teacher)

Yes or no

3497 Now complete the following sentence. Remember, there is no right or wrong answer! 
$3500 \quad$ Your bowel be empty before the examination

3501 must should

will has to

3502 When you arrive you go to the reception desk in the department

3503 need to must should have to

3504 If you stay in the department then you use the special toilet for 3505 nuclear medicine patients.

3506 need to have to must should

3507 Some diseases such as colds trigger an asthma attack.

3508 may can will

3509 You __ smoke after midnight the day before the procedure.

3510 should not must not can not do not

3511 You __ tell the radiographer if you have breast implants.

3512 must should have to need to

3513 There is a very small risk that inflating the colon with air injure or 3514 perforate the bowel

3515 could might will can

3516 During the exam, you __ lie still, but breathe normally as you move 3517 through the scanner.

3518 have to must should need to 
3519 Women always inform their physician and $x$-ray technologist if there

3520 is any possibility that they are pregnant

3521 must should have to need to

3522 If you have any allergies you tell the radiology staff before you have

3523 the examination.

3524 need to have to must should

3525 You _ eat or drink anything 6 to 12 hours before the procedure

3526 must not can not should not ought not to

3527 You ___ fast before the procedure.

3528 need to should must have to

3529 Some patients ___ take antibiotics before the procedure

3530 have to need to must should

3531 If you go home after the exam, you ___ arrange for someone to take you

3532 home by car or taxi and to stay with you overnight.

3533 must need to should have to

3534 Many things_____ead to the inflammation of the lungs and abnormal

3535 muscle tightening, these are known as triggers.

3536 might can could will

3537 In diabetic neuropathy, your feet or legs ___ feel numb or unusually cold.

3538 could might should will

3539 Risks and complications are very unlikely, but possible. You ___ know

3540 about them just in case they happen.

3541 should must need to have to 
3542 Your visit to the clinic mean spending two to three hours in the

3543 department

3544 will could may can

3545 If you_________encel your appointment or change the date or time, please

3546 call us on the number below.

3547 have to must ---- need to

3548 You ___ stay in hospital for up to four hours after the procedure for us

3549 to observe you.

3550 have to should need to must

3551

3552

3553

3554

3555

3556

3557

3558

3559

3560

3561

3562

3563 


\section{References}

3568

3569

3570

3571

3572

3573

3574

3575

3576

3577

3578

3579

3580

3581

3582

3583

3584

3585

3586

Ädel, A., \& Erman, B. (2012). Recurrent word combinations in academic writing by native and non-native speakers of English: A lexical bundles approach. http://doi.org/10.1016/j.esp.2011.08.004

Adepu, R., \& Swamy, M. K. (2012). Development and Evaluation of Patient Information Leaflets (PIL) Usefulness. Indian Journal of Pharmaceutical Sciences, 74(2), 174-8. http://doi.org/10.4103/0250-474X.103857

Adolphs, S., Brown, B., Carter, R., Crawford, P., \& Sahota, O. (2004). Applying corpus linguistics in a health care context. Journal of Applied Linguistics, 1(1), 9-28. http://doi.org/10.1558/japl.1.1.9.55871

Al-Harthy, N., Sudersanadas, K. M., Al-Mutairi, M., Vasudevan, S., Bin Saleh, G., Al-Mutairi, M., \& Hussain, L. W. (2016). Efficacy of patient discharge instructions: A pointer toward caregiver friendly communication methods from pediatric emergency personnel. Journal of Family \& Community Medicine, 23(3), 155-60. http://doi.org/10.4103/2230-8229.189128

Allan, R., \& Se, R. A. (2017). From Do You Know to I Don't Know: An Analysis of the Frequency and Usefulness of Lexical Bundles in Five English Language SelfStudy Books. Corpus Pragmatics, 1, 351-372. http://doi.org/10.1007/s41701017-0016-9

Angouri, J., \& Sanderson, T. (2016). "You'll find lots of help here" unpacking the function of an online Rheumatoid Arthritis (RA) forum. Language and Communication, 46, 1-13. http://doi.org/10.1016/j.langcom.2015.10.001

Antaki, C., \& Kent, A. (2012). Telling people what to do (and, sometimes, why): Contingency, entitlement and explanation in staff requests to adults with intellectual impairments. Journal of Pragmatics, 44(6-7), 876-889. http://doi.org/10.1016/j.pragma.2012.03.014

Askehave, I. (1999). Askehave-Communicative Purpose as Genre Determinant. Journal of Linguistics, 23(23), 13-24. Retrieved from https://pure.au.dk/ws/files/9940/H23_02.pdf

Askehave, I., \& Zethsen, K. K. (2014). A comparative analysis of the lay-friendliness of Danish EU patient information leaflets from 2000 to 2012. Communication and Medicine. http://doi.org/10.1558/cam.v11i3.20700 
3602

3603

3604

3605

3606

3607

3608

3609

3610

3611

3612

3613

3614

3615

3616

3617

3618

3619

3620

3621

3622

3623

3624

3625

3626

3627

3628

3629

3630

3631

3632

3633

3634

3635

3636

Atkinson, D. (1992). The Evolution of Medical Research Writing from 1735 to 1985: The Case of the Edinburgh Medical Journal. Retrieved from http://applij.oxfordjournals.org/

Azam, N., \& Harrison, M. (2011). Patients' perspectives on injuries. Emergency Medicine Journal : EMJ, 28(7), 601-3. http://doi.org/10.1136/emj.2009.082032

Badarudeen, S., \& Sabharwal, S. (2010). Assessing readability of patient education materials: Current role in orthopaedics. Clinical Orthopaedics and Related Research, 468(10), 2572-2580. http://doi.org/10.1007/s11999-010-1380-y

Bagley, C., Hunter, R., \& Bacarese-Hamilton, I. (2011, July). Patients' misunderstanding of common orthopaedic terminology: the need for clarity. Annals of the Royal College of Surgeons of England. http://doi.org/10.1308/003588411X580179

Baker, D., \& Robson, J. (2012). Communication training for international graduates. The Clinical Teacher, 9(5), 325-9. http://doi.org/10.1111/j.1743498X.2012.00555.x

Barbieri, F. (2018). I don't want to and don't get me wrong. In J. Kopaczyk \& Tyrkko Jukka (Eds.), Applications of pattern driven methods in corpus linguistics (pp. 251-276). Amsterdam: John Benjamins Publishing Company. http://doi.org/10.1075/scl.82.10bar

Barnes, R. K., Jepson, M., Thomas, C., Jackson, S., Metcalfe, C., Kessler, D., \& Cramer, H. (2018). Using conversation analytic methods to assess fidelity to a talk-based healthcare intervention for frequently attending patients. Social Science \& Medicine , 206, 38-50. http://doi.org/10.1016/j.socscimed.2018.04.008

Bartels, R. D., Kelly, K. M., \& Rothman, A. J. (2010). Moving beyond the function of the health behaviour: The effect of message frame on behavioural decisionmaking. Psychology and Health, 25(7), 821-838. http://doi.org/10.1080/08870440902893708

Baumann, B. M., Chen, E. H., Mills, A. M., Glaspey, L., Thompson, N. M., Jones, M. K., \& Farner, M. C. (2010). Patient Perceptions of Computed Tomographic Imaging and Their Understanding of Radiation Risk and Exposure. YMEM, 58, 1-7.e2. http://doi.org/10.1016/j.annemergmed.2010.10.018

Bellés-Fortuño, B. (2018). Multimodality in medicine: How university medical students approach informative leaflets. System, 77, 28-38. http://doi.org/10.1016/J.SYSTEM.2018.02.012

Berkhof, M., van Rijssen, H. J., Schellart, A. J. M., Anema, J. R., \& van der Beek, A. J. (2011). Effective training strategies for teaching communication skills to physicians: An overview of systematic reviews. Patient Education and Counseling, 84(2), 152-162. http://doi.org/10.1016/j.pec.2010.06.010 
Berry, D., Knapp, P., \& Raynor, D. (2002). Provision of information about drug sideeffects to patients. The Lancet, 359(9309), 853-854. http://doi.org/10.1016/S0140-6736(02)07923-0

Berry, D. C., Knapp, P., \& Raynor, T. (2006). Expressing medicine side effects: Assessing the effectiveness of absolute risk, relative risk, and number needed to harm, and the provision of baseline risk information. Patient Education and Counseling, 63(1-2), 89-96. http://doi.org/10.1016/J.PEC.2005.09.003

Bianco, A. V. (2016). VAGHEZZA, INDETERMINATEZZA E INCERTEZZA NEI FOGLIETTI ILLUSTRATIVI DEI MEDICINALI IN INGLESE E IN ITALIANO. http://doi.org/10.1285/i22390359v19p41

Biber, D. D., Conrad, S., Reppen, R., Byrd, P., \& Helt, M. (2002). Speaking and Writing in the University: A Multidimensional Comparison. TESOL Quarterly, 36(1), 8-48. http://doi.org/10.2307/3588359

Biber, D. (2012). Register as a Predictor of Linguistic Variation. Corpus Linguistics and Linguistic Theory, 8(1), 9-37. http://doi.org/10.1515/cllt-2012-0002

Biber, D. (1988). Variation across speech and writing. Cambridge University Press. http://doi.org/10.1017/CBO9780511621024

Biber, D., \& Conrad, S. (2009). Register, Genre, and Style. Cambridge University Press. http://doi.org/10.1017/CBO9780511814358

Biber, D., Conrad, S., \& Reppen, R. (1998). Corpus linguistics : investigating language structure and use. Cambridge University Press. Retrieved from https://books.google.es/books?hl=en\&lr=\&id=2h5F7TXa6psC\&oi=fnd\&pg=PR9 $\& \mathrm{dq}=$ Corpus+linguistics\&ots $=8 \mathrm{QmbvuynjA \& sig=KG6umOH2ZARVgNkiulDa}$ $\mathrm{kHqvMBw} \&$ redir_esc $=\mathrm{y} \# \mathrm{v}=$ onepage $\& \mathrm{q}=$ Corpus linguistics $\& \mathrm{f}=\mathrm{false}$

Biber, D., Johansson, S., Leech, G., Conrad, S., \& Finegan, E. (1999). Longman Grammar of Spoken and Written English. Longman. http://doi.org/10.2307/3587792

Biber, D., \& Barbieri, F. (2007). Lexical bundles in university spoken and written registers. English for Specific Purposes, 26(3), 263-286. http://doi.org/10.1016/j.esp.2006.08.003

Biber, D., Conrad, S., \& Cortes, V. (2004). If you look at ...: Lexical bundles in university teaching and textbooks. Applied Linguistics. http://doi.org/10.1093/applin/25.3.371

Biber, D., Conrad, S., \& Reppen, R. (1996). Corpus-Based Investigations of Language Use. Annual Review of Applied Linguistics, 16, 115. http://doi.org/10.1017/S0267190500001471 
Biber, D., \& Finegan, E. (2001). Intra-textual variation within medical research articles. In ed. by N. O. and P. de Haan (Ed.), Variation in English: Multidimensional Studies (pp. 108-123). Rodopi.

Bidmon, S., \& Terlutter, R. (2015). Gender differences in searching for health information on the internet and the virtual patient-physician relationship in Germany: Exploratory results on how men and women differ and why. Journal of Medical Internet Research, 17(6), e156. http://doi.org/10.2196/jmir.4127

Blackman, J., \& Sahebjalal, M. (2014). Patient understanding of frequently used cardiology terminology. British Journal of Cardiology, 21(1), 39. http://doi.org/10.5837/bjc.2014.007

Blalock, S. J., Sage, A., Bitonti, M., Patel, P., Dickinson, R., \& Knapp, P. (2016). Communicating information concerning potential medication harms and benefits: What gist do numbers convey? Patient Education and Counseling, 99(12), 19641970. http://doi.org/10.1016/j.pec.2016.07.022

Blomberg, F., Brulin, C., Andertun, R., \& Rydh, A. (2010). Patients' Perception of Quality of Care in a Radiology Department: A Medical-Physical Approach. Journal of Radiology Nursing, 29(1), 10-17. http://doi.org/10.1016/j.jradnu.2009.09.001

Bolderston, A., Palmer, C., Flanagan, W., \& McParland, N. (2008). The experiences of English as second language radiation therapy students in the undergraduate clinical program: Perceptions of staff and students. Radiography, 14(3), 216225. http://doi.org/10.1016/j.radi.2007.03.006

Booth, L. A., \& Manning, D. J. (2006). Observations of radiographer communication: An exploratory study using Transactional Analysis. Radiography, 12(4), 276282. http://doi.org/10.1016/j.radi.2005.09.005

Bostock, S., \& Steptoe, A. (2012). Association between low functional health literacy and mortality in older adults: longitudinal cohort study. BMJ (Clinical Research $E d$.), 344, e1602. Retrieved from http://www.ncbi.nlm.nih.gov/pubmed/22422872

Boundouki, G., Humphris, G., \& Field, A. (2004). Knowledge of oral cancer, distress and screening intentions: longer term effects of a patient information leaflet. Patient Education and Counseling, 53(1), 71-7. http://doi.org/10.1016/S07383991(03)00118-6

Bourhis, R. Y., Roth, S., \& MacQueen, G. (1989). Communication in the hospital setting: a survey of medical and everyday language use amongst patients, nurses and doctors. Social Science \& Medicine (1982), 28(4), 339-46. Retrieved from http://www.ncbi.nlm.nih.gov/pubmed/2705006 
Boyle, C. M. (1970). Difference between patients' and doctors' interpretation of some common medical terms. British Medical Journal, 2(5704), 286-289. http://doi.org/10.1136/bmj.2.5704.286

Buckton, C. H., Lean, M. E. J., \& Combet, E. (2015). 'Language is the source of misunderstandings'-impact of terminology on public perceptions of health promotion messages. BMC Public Health, 15(1), 579. http://doi.org/10.1186/s12889-015-1884-1

Budgell, B. (n.d.). Titles of Biomedical Articles: a corpus-based analysis. Retrieved from www.icmje.org

Burton, A. K., Waddell, G., Tillotson, K. M., \& Summerton, N. (1999). Information and advice to patients with back pain can have a positive effect. A randomized controlled trial of a novel educational booklet in primary care. Spine, 24(23), 2484-91. Retrieved from http://www.ncbi.nlm.nih.gov/pubmed/10626311

Campbell, D. A., \& Johnson, S. B. (2001). Comparing syntactic complexity in medical and non-medical corpora. Proceedings / AMIA ... Annual Symposium. AMIA Symposium, 90-4. Retrieved from http://www.pubmedcentral.nih.gov/articlerender.fcgi?artid=2243419\&tool=pmce ntrez\&rendertype $=$ abstract

Candlin, C. N., \& Candlin, S. (2003). HEALTH CARE COMMUNICATION: A PROBLEMATIC SITE FOR APPLIED LINGUISTICS RESEARCH. Annual Review of Applied Linguistics, 23(134). Retrieved from https://www.researchonline.mq.edu.au/vital/access/services/Download/mq:5617/ DS01? view=true

Candlin, S. (2003). Issues Arising when the Professional Workplace is the Site of Applied Linguistic Research. Applied Linguistics, 24(3), 386-394. http://doi.org/10.1093/applin/24.3.386

Carnet, D., \& Magnet, A. (2006). Medical Journals, Letters to the Editor. In K. Brown (Ed.), Encyclopedia of Language and Lingiustics, (2nd ed., pp. 725-732). Elsevier Science.

Carter, R. A., \& McCarthy, M. J. (1995). Grammar and the Spoken Language.pdf. Applied Linguistics, 16, 141-158.

Chang, M. Y., Kim, J.-W., \& Rhee, C.-S. (2015). The quality of health information on allergic rhinitis, rhinitis, and sinusitis available on the internet. Allergy, Asthma \& Immunology Research, 7(2), 141-7. http://doi.org/10.4168/aair.2015.7.2.141

Chapman, K., Abraham, C., Jenkins, V., \& Fallowfield, L. (2003). Lay understanding of terms used in cancer consultations. Psycho-Oncology, 12(6), 557-566. http://doi.org/10.1002/pon.673 
Charnock, D., Shepperd, S., Needham, G., \& Gann, R. (1999). DISCERN: An instrument for judging the quality of written consumer health information on treatment choices. Journal of Epidemiology and Community Health, 53(2), 105111. http://doi.org/10.1136/jech.53.2.105

Chen, Q., \& Ge, G. (2007). A corpus-based lexical study on frequency and distribution of Coxhead's AWL word families in medical research articles (RAs). English for Specific Purposes, 26(4), 502-514. http://doi.org/10.1016/j.esp.2007.04.003

Cherla, D. V., Sanghvi, S., Choudhry, O. J., Liu, J. K., \& Eloy, J. A. (2012). Readability assessment of Internet-based patient education materials related to endoscopic sinus surgery. The Laryngoscope, 122(8), 1649-54. http://doi.org/10.1002/lary.23309

Chin-Mo, C., \& Yu-Chin, C. (2011). EU opt in and out under the framework of Lisbon treaty. Tamkang Journal of International Affairs, 14(3), 95-128. Retrieved from http://dialnet.unirioja.es/servlet/dcart?info=link\&codigo=2475568\&orden=14089 1

Chu, J. T., Wang, M. P., Shen, C., Viswanath, K., Lam, T. H., \& Chan, S. S. C. (2017). How, When and Why People Seek Health Information Online: Qualitative Study in Hong Kong. Interactive Journal of Medical Research, 6(2), e24. http://doi.org/10.2196/ijmr.7000

Chur-Hansen, A., \& Barrett, R. J. (1996). Teaching colloquial Australian English to medical students from non-English speaking backgrounds. Medical Education, 30(6), 412-417. http://doi.org/10.1111/j.1365-2923.1996.tb00860.x

Chur-Hansen, A., Vernon-Roberts, J., \& Clark, S. (1997). Language background, English language proficiency and medical communication skills of medical students. Medical Education, 31(4), 259-263. http://doi.org/10.1111/j.13652923.1997.tb02922.x

Cilveti, L. D., Pérez, I. K. L., La, U. De, \& Tenerife, L. (n.d.). Textual and language flaws : problems for Spanish doctors in producing abstracts in English, 61-79.

Claramita, M., Utarini, A., Soebono, H., Van Dalen, J., \& Van der Vleuten, C. (2011). Doctor-patient communication in a Southeast Asian setting: the conflict between ideal and reality. Advances in Health Sciences Education, 16(1), 69-80. http://doi.org/10.1007/s10459-010-9242-7

Clerehan, R., \& Buchbinder, R. (2006). Toward a more valid account of functional text quality: The case of the patient information leaflet. Text and Talk. http://doi.org/10.1515/TEXT.2006.003

Clerehan, R., Buchbinder, R., \& Moodie, J. (2005). A linguistic framework for assessing the quality of written patient information: Its use in assessing 
methotrexate information for rheumatoid arthritis. Health Education Research, 20(3), 334-344. http://doi.org/10.1093/her/cyg123

Close, J., \& Aarts, B. (2008). Changes in the use of the modals HAVE TO, HAVE GOT TO and MUST. Retrieved from https://www.ucl.ac.uk/englishusage/projects/verb-phrase/Close and Aarts ICEHL 15.pdf

Collins, P. (2009). Modals and quasi-modals in $\{\mathrm{W}\}$ orld $\{\mathrm{E}\}$ nglishes. World Englishes, 28(3), 281-292. Retrieved from https://books.google.ch/books?id=GIDgo3L1MEsC\&source=gbs_slider_cls_met adata_1_mylibrary

Collins, S. (2005). Explanations in consultations: the combined effectiveness of doctors' and nurses' communication with patients. Medical Education, 39(8), 785-796. http://doi.org/10.1111/j.1365-2929.2005.02222.x

Connor, U., Antón, M., Goering, E., Lauten, K., Roach, P., Balunda, S., \& Hayat, A. (2013). Listening to patients' voices: Linguistic indicators related to diabetes self-management. Communication \& Medicine, $9(1)$. http://doi.org/10.1558/cam.v9i1.1

Conrad, S. (2015). Register variation. In The Cambridge Handbook of English Corpus Linguistics (pp. 309-329). http://doi.org/10.1007/9781139764377.018

Conrad, S., \& Biber, D. (2005). The Frequency and Use of Lexical Bundles in Conversation and Academic Prose. Lexicographica, 20/2004, 56-71. http://doi.org/10.1515/9783484604674.56

Cortes, V. (2004). Lexical bundles in published and student disciplinary writing: Examples from history and biology. English for Specific Purposes, 23(4), 397423. http://doi.org/10.1016/J.ESP.2003.12.001

Cortes, V. (2006). Teaching lexical bundles in the disciplines: An example from a writing intensive history class. Linguistics and Education, 17(4), 391-406. http://doi.org/10.1016/J.LINGED.2007.02.001

Cortes, V. (2013). The purpose of this study is to: Connecting lexical bundles and moves in research article introductions. Journal of English for Academic Purposes, 12(1), 33-43. http://doi.org/10.1016/J.JEAP.2012.11.002

Coulter, A., \& Ellins, J. (2007). Effectiveness of strategies for informing, educating, and involving patients. BMJ (Clinical Research Ed.), 335(7609), 24-7. http://doi.org/10.1136/bmj.39246.581169.80

Crawford, P., \& Brown, B. (2011). Fast healthcare: Brief communication, traps and opportunities. Patient Education and Counseling, 82(1), 3-10. http://doi.org/10.1016/j.pec.2010.02.016 
Cross, C., \& Oppenheim, C. (2006). A genre analysis of scientific abstracts. Journal of Documentation, 62(4), 428-446. http://doi.org/10.1108/00220410610700953

3824 Crosthwaite, P., Cheung, L., \& Jiang, F. (Kevin). (2017). Writing with attitude: Stance expression in learner and professional dentistry research reports. English for Specific Purposes, 46. http://doi.org/10.1016/j.esp.2017.02.001

Csomay, E. (2013). Lexical Bundles in Discourse Structure: A Corpus-Based Study of Classroom Discourse. Applied Linguistics, 34(3), 369-388. http://doi.org/10.1093/applin/ams045

Curtis, J. R., Back, A. L., Ford, D. W., Downey, L., Shannon, S. E., Doorenbos, A. Z., ... Engelberg, R. A. (2013). Effect of communication skills training for residents and nurse practitioners on quality of communication with patients with serious illness: a randomized trial. JAMA, 310(21), 2271-81. http://doi.org/10.1001/jama.2013.282081 Explored. http://doi.org/10.1057/9780230627420

Dahm, M. R. (2011). Exploring perception and use of everyday language and medical terminology among international medical graduates in a medical ESP course in Australia. English for Specific Purposes, 30(3), 186-197. http://doi.org/10.1016/j.esp.2011.02.004

Dahm, M. R. (2018). A socio-cognitive investigation of English medical terminology: dynamic varieties of meaning. Lexicography, 4(1), 81-103. http://doi.org/10.1007/s40607-018-0039-9

Dahm, M. R., \& Yates, L. (2013). English for the Workplace: Doing Patient-Centred Care in Medical Communication. TESL Canada Journal, 30(7), 21. Retrieved from http://teslcanadajournal.ca/index.php/tesl/article/view/1150 international medical graduates' communication: The contribution of applied linguistics. Medical Education, 49(8), 828-837. http://doi.org/10.1111/medu.12776

Davey, H. M., Butow, P. N., \& Armstrong, B. K. (2003). Cancer patients' preferences for written prognostic information provided outside the clinical context. British Journal of Cancer, 89(8), 1450-6. http://doi.org/10.1038/sj.bjc.6601287

De Bont, E. G. P. M., Alink, M., Falkenberg, F. C. J., Dinant, G. J., \& Cals, J. W. L. (2015). Patient information leaflets to reduce antibiotic use and reconsultation rates in general practice: A systematic review. BMJ Open, 5(6), e007612e007612. http://doi.org/10.1136/bmjopen-2015-007612 
DeCarrico, J., \& Nattinger, J. R. (1988). Lexical phrases for the comprehension of academic lectures. English for Specific Purposes, 7(2), 91-102. http://doi.org/10.1016/0889-4906(88)90027-0

Demark-Wahnefried, W., Peterson, B., McBride, C., Lipkus, I., \& Clipp, E. (2000). Current health behaviors and readiness to pursue life-style changes among men and women diagnosed with early stage prostate and breast carcinomas. Cancer, 88(3), 674-684. http://doi.org/10.1002/(SICI)10970142(20000201)88:3<674::AID-CNCR26>3.0.CO;2-R

Diaz, J. A., Griffith, R. A., Ng, J. J., Reinert, S. E., Friedmann, P. D., \& Moulton, A. W. (2002). Patients' use of the Internet for medical information. Journal of General Internal Medicine, 17(3), 180-5. http://doi.org/10.1046/J.15251497.2002.10603.X

Dickinson, D., Raynor, D. K., \& Duman, M. (2001). Patient information leaflets for medicines: using consumer testing to determine the most effective design. Patient Education and Counseling, 43(2), 147-59. Retrieved from http://www.ncbi.nlm.nih.gov/pubmed/11369148

Dieltjens, S. M., Heynderickx, P. C., Dees, M. K., \& Vissers, K. C. (2014). Linguistic Analysis of Face-to-Face Interviews with Patients with An Explicit Request for Euthanasia, their Closest Relatives, and their Attending Physicians: the Use of Modal Verbs in Dutch. Pain Practice, 14(4), 324-331. http://doi.org/10.1111/papr.12076

Dixon-Woods, M. (2001). Writing wrongs? An analysis of published discourses about the use of patient information leaflets. Social Science \& Medicine, 52(9), 14171432. http://doi.org/10.1016/S0277-9536(00)00247-1

Dong, S., Butow, P. N., Costa, D. S. J., Dhillon, H. M., \& Shields, C. G. (2014). The influence of patient-centered communication during radiotherapy education sessions on post-consultation patient outcomes. Patient Education and Counseling, 95(3), 305-312. http://doi.org/10.1016/j.pec.2014.02.008

Douglas, B. (2006). Stance in spoken and written university registers. Journal of English for Academic Purposes, 5(2), 97-116. http://doi.org/10.1016/j.jeap.2006.05.001

Drew, P., Chatwin, J., \& Collins, S. (2001). Conversation analysis: a method for research into interactions between patients and health-care professionals. Health Expectations, 4(1), 58-70. http://doi.org/10.1046/j.1369-6513.2001.00125.x

Durrant, P. (2017). Lexical Bundles and Disciplinary Variation in University Students' Writing: Mapping the Territories. Applied Linguistics, 38(2), 165-193. http://doi.org/10.1093/applin/amv011 
Dutta-Bergman, M. (2003). Trusted online sources of health information: differences in demographics, health beliefs, and health-information orientation. Journal of Medical Internet Research, 5(3), e21. http://doi.org/10.2196/jmir.5.3.e21

Eheman, C. R., Berkowitz, Z., Lee, J., Mohile, S., Purnell, J., Rodriguez, E. M., ... Morrow, G. (2009). Information-seeking styles among cancer patients before and after treatment by demographics and use of information sources. Journal of Health Communication, 14(5), 487-502. http://doi.org/10.1080/10810730903032945

Ehrlich, R. A., \& Coakes, D. M. (2017). Patient Care in Radiography: With an Introduction to Medical Imaging. Retrieved from https://books.google.ch/books/about/Patient_Care_in_Radiography.html?id=A55 QPgAACAAJ\&pgis $=1$

Ek, S. (2013). Gender differences in health information behaviour: a Finnish population-based survey. Health Promotion Internation. http://doi.org/10.1093/heapro/dat063

Esfandiari, R., \& Barbary, F. (2017). A contrastive corpus-driven study of lexical bundles between English writers and Persian writers in psychology research articles. Journal of English for Academic Purposes, 29, 21-42. http://doi.org/10.1016/j.jeap.2017.09.002

Eysenbach, G., \& Köhler, C. (2002). How do consumers search for and appraise health information on the world wide web? Qualitative study using focus groups, usability tests, and in-depth interviews. Bmj, 324(7337), 573-577. http://doi.org/10.1136/bmj.324.7337.573

Fage-Butler, A. (2011). The discursive construction of risk and trust in patient information leaflets. Hermes, 46, 61-74. Retrieved from www.medicines.org.uk

Fage-Butler, A. (2013). Improving patient information leaflets: Developing and applying an evaluative model of patient-centredness for text. Communication and Medicine, 10(2), 105-115. http://doi.org/10.1558/cam.v10i2.105

Fage-Butler, A. M., \& Nisbeth Jensen, M. (2016). Medical terminology in online patient-patient communication: evidence of high health literacy? Health Expectations : An International Journal of Public Participation in Health Care and Health Policy, 19(3), 643-53. http://doi.org/10.1111/hex.12395

Fakult, P., Seggewi, F., \& Ss, H. (2012). Current Changes in the English Modals - a Corpus-Based Analysis of Present-Day Spoken English.

Fehringer C, C. K. (2015). " You' ve got to sort of eh hoy the Geordie out ": Modals of obligation and necessity in 50 years of Tyneside English . English Language and Linguistics 2015 , 19 ( Special Issue 2 ), 355-381 . Copyright : Date deposited :, 19(2), 355-381. 
3933

3934

3935

3936

3937

3938

3939

3940

3941

3942

3943

3944

3945

3946

3947

3948

3949

3950

3951

3952

3953

3954

3955

3956

3957

3958

3959

3960

3961

3962

3963

3964

3965

3966

3967

3968

Ferguson, G. (2001). If you pop over there: a corpus-based study of conditionals in medical discourse. English for Specific Purposes, 20(1), 61-82. http://doi.org/10.1016/S0889-4906(99)00027-7

Fields, A. M., Freiberg, C. S., Fickenscher, A., \& Shelley, K. H. (2008). Patients and jargon: are we speaking the same language? Journal of Clinical Anesthesia, 20(5), 343-346. http://doi.org/10.1016/j.jclinane.2008.02.006

Fleischman, S. (2008). Language and Medicine. In The Handbook of Discourse Analysis (Vol. 19, pp. 470-502). Cambridge University Press. http://doi.org/10.1002/9780470753460.ch25

Flight, I. H., Wilson, C. J., Zajac, I. T., Hart, E., \& McGillivray, J. A. (2012). Decision Support and the Effectiveness of Web-based Delivery and Information Tailoring for Bowel Cancer Screening: An Exploratory Study. JMIR Research Protocols, 1(2), e12. http://doi.org/10.2196/resprot.2135

Flowerdew, J. (2015). John Swales's approach to pedagogy in Genre Analysis: A perspective from 25 years on. Journal of English for Academic Purposes, 19, 102-112. http://doi.org/10.1016/j.jeap.2015.02.003

Flowerdew, L. (2005). An integration of corpus-based and genre-based approaches to text analysis in EAP/ESP: Countering criticisms against corpus-based methodologies. English for Specific Purposes, 24(3), 321-332. http://doi.org/10.1016/j.esp.2004.09.002

Fox, N. J., Ward, K. J., \& O'rourke, A. J. (2005). The "expert patient": empowerment or medical dominance? The case of weight loss, pharmaceutical drugs and the Internet. Social Science \& Medicine, 60, 1299-1309. http://doi.org/10.1016/j.socscimed.2004.07.005

Fox, N., \& Ward, K. (2006). Health identities: from expert patient to resisting consumer. Health: An Interdisciplinary Journal for the Social Study of Health, Illness and Medicine, 10(4), 461-479. http://doi.org/10.1177/1363459306067314

Frank, R. A. (2000). Medical communication: non-native English speaking patients and native English speaking professionals. English for Specific Purposes, 19(1), 31-62. http://doi.org/10.1016/S0889-4906(98)00012-X

Friedman, D. B., \& Hoffman-Goetz, L. (2006). A Systematic Review of Readability and Comprehension Instruments Used for Print and Web-Based Cancer Information. Health Education \& Behavior, 33(3), 352-373. http://doi.org/10.1177/1090198105277329

Fuertes Olivera, P. a. (2008). Advances in Medical Discourse Analysis: Oral and Written Contexts. Iberica, 15(2), 186-191. 
3969

3970

3971

3972

3973

3974

3975

3976

3977

3978

3979

3980

3981

3982

3983

3984

3985

3986

3987

3988

3989

3990

3991

3992

3993

3994

3995

3996

3997

3998

3999

4000

4001

4002

4003

4004

4005

4006

Gaissmaier, W., \& Gigerenzer, G. (2008). Statistical illiteracy undermines informed shared decision making. Zeitschrift Fur Evidenz, Fortbildung Und Qualitat Im Gesundheitswesen, 102(7), 411-413. http://doi.org/10.1016/j.zefq.2008.08.013

Gajšt, N. (2014). English Modal Verbs in Modal Verb Phrase Structures and Lexical Associations of Modality in 'Standard Terms and Conditions of Sale.' Journal for Foreign Languages, 4(1-2), 243. http://doi.org/10.4312/vestnik.4.243-260

Gamble, M. (2014). Medical Malpractice in America: 15 Latest Statistics. Retrieved November 17, 2018, from https://www.beckershospitalreview.com/legalregulatory-issues/medical-malpractice-in-america-15-latest-statistics.html

Garner, M., Ning, Z., \& Francis, J. (2012). A framework for the evaluation of patient information leaflets. Health Expectations : An International Journal of Public Participation in Health Care and Health Policy, 15(3), 283-294. http://doi.org/10.1111/j.1369-7625.2011.00665.x

Gasiorek, J., Van de Poel, K., \& Blockmans, I. (2015). What do you do when you can't accommodate? Managing and evaluating problematic interactions in a multilingual medical environment. Language \& Communication, 41, 84-88. http://doi.org/10.1016/j.langcom.2014.10.005

Gaspari, F. (2013). A phraseological comparison of international news agency reports published online: Lexical bundles in the English-language output of ANSA, Adnkronos, Reuters and UPI. VARIENG, 13.

Gelb, K., Pederson, A., \& Greaves, L. (2012). How have health promotion frameworks considered gender? Health Promotion International, 27(4), 445-452. http://doi.org/10.1093/heapro/dar087

Gemoets, D., Rosemblat, G., Tse, T., \& Logan, R. (2004). Assessing readability of consumer health information: An exploratory study. Studies in Health Technology and Informatics, 107, 869-873. http://doi.org/10.3233/978-1-60750949-3-869

Ghafar Samar, R., Shokrpour, N., \& Nasiri, E. (2018). The Applicability of Teaching Lexical Bundles on Medical Students' Writing Proficiency in an EFL Context. Teaching English Language, 12(2), 27-44. http://doi.org/10.22132/TEL.2018.70728

Gillies, K., Huang, W., Skea, Z., Brehaut, J., \& Cotton, S. (2014). Patient information leaflets (PILs) for UK randomised controlled trials: A feasibility study exploring whether they contain information to support decision making about trial participation. Trials, 15(1). http://doi.org/10.1186/1745-6215-15-62

Gilmore, A., \& Millar, N. (2018). The language of civil engineering research articles: A corpus-based approach. English for Specific Purposes, 51, 1-17. http://doi.org/10.1016/J.ESP.2018.02.002 
4007

4008

4009

4010

4011

4012

4013

4014

4015

4016

4017

4018

4019

4020

4021

4022

4023

4024

4025

4026

4027

4028

4029

4030

4031

4032

4033

4034

4035

4036

4037

4038

4039

4040

4041

4042

4043

Giménez-Moreno, R., \& Skorczynska, H. (2013). Corpus Analysis and Register Variation: A Field in Need of an Update. Procedia - Social and Behavioral Sciences, 95, 402-408. http://doi.org/10.1016/J.SBSPRO.2013.10.662

Gisborne, N. (2007). Dynamic Modality. Journal of Theoretical Linguistics, 4(2), 4461.

Glass, L. (2015). Grounding the social meaning of a strong necessity modal in its semantics (pp. 1-25).

Gledhill, C. (1995). Scientific innovation and the phraseology of rhetoric: Posture, reformulation and collocation in cancer research articles (Vol. Dissertati). Retrieved from http://www.isfla.org/Systemics/Print/Theses/Gledhill1995.pdf

Godolphin, W., Towle, A., \& McKendry, R. (2001). Evaluation of the quality of patient information to support informed shared decision-making. Health Expectations : An International Journal of Public Participation in Health Care and Health Policy, 4(4), 235-42. Retrieved from http://www.ncbi.nlm.nih.gov/pubmed/11703497

Gooberman-Hill, R., French, M., Dieppe, P., \& Hawker, G. (2009). Expressing pain and fatigue: A new method of analysis to explore differences in osteoarthritis experience. Arthritis Care and Research, 61(3), 353-360. http://doi.org/10.1002/art.24273

Goodwin, J. S. (2002). Language and Medicine: Thinking and Talking About Alzheimer's Disease. The Gerontologist, 42(3), 293-296. http://doi.org/10.1093/geront/42.3.293

Gotti, M. (2015). Insights into medical discourse: diachronic and synchronic perspectives. Esp Today-Journal of English for Specific Purposes at Tertiary Level, 3(1), 5-24. Retrieved from https://aisberg.unibg.it/retrieve/handle/10446/42776/44283/Gotti - Insights into medical discourse.pdf

Gozdz-Roszkowski, S. (2011). Patterns of linguistic variation in American legal English : a corpus-based study. (Vol. 22). Frankfurt am Main: Peter Lang.

Grabowski, Ł. (2013). Register Variation Across English Pharmaceutical Texts: A Corpus-driven Study of Keywords, Lexical Bundles and Phrase Frames in Patient Information Leaflets and Summaries of Product Characteristics. Procedia Social and Behavioral Sciences, 95, 391-401. http://doi.org/10.1016/j.sbspro.2013.10.661

Grabowski, Ł. (2014). On Lexical Bundles in Polish Patient Information Leaflets: A Corpus-Driven Study. Http://Www.Ejournals.Eu/SPL/, Volume 9(Issue 1), 21-43. http://doi.org/10.4467/23005920SPL.14.002.2186 
4044

4045

4046

4047

4048

4049

4050

4051

4052

4053

4054

4055

4056

4057

4058

4059

4060

4061

4062

4063

4064

4065

4066

4067

4068

4069

4070

4071

4072

4073

4074

4075

4076

4077

4078

4079

4080

4081

4082

Grabowski, Ł. (2015). Keywords and lexical bundles within English pharmaceutical discourse: A corpus-driven description. English for Specific Purposes, 38, 23-33. http://doi.org/10.1016/j.esp.2014.10.004

Granhagen Jungner, J., Tiselius, E., Wenemark, M., Blomgren, K., Lützén, K., \& Pergert, P. (2018). Development and evaluation of the Communication over Language Barriers questionnaire (CoLB-q) in paediatric healthcare. Patient Education and Counseling, 101(9), 1661-1668. http://doi.org/10.1016/j.pec.2018.04.007

Greaves, F., Ramirez-cano, D., Millett, C., Darzi, A., \& Donaldson, L. (2010). Machine learning and sentiment analysis of unstructured free-text information about patient experience online, 2010.

Grime, J. C., \& Ong, B. N. (2007). Constructing osteoarthritis through discourse - A qualitative analysis of six patient information leaflets on osteoarthritis. $B M C$ Musculoskeletal Disorders, 8(1), 34. http://doi.org/10.1186/1471-2474-8-34

Grime, J., \& Pollock, K. (2004). Information versus experience: A comparison of an information leaflet on antidepressants with lay experience of treatment. Patient Education and Counseling, 54(3), 361-368. http://doi.org/10.1016/j.pec.2004.02.003

Hadjipavlou, M., Khan, S., \& Rane, a. (2013). Readability of patient information leaflets for urological conditions and treatments. Journal of Clinical Urology, 6(5), 302-305. http://doi.org/10.1177/2051415813489554

Hadlow, J., \& Pitts, M. (1991). The understanding of common health terms by doctors, nurses and patients. Social Science \& Medicine (1982), 32(2), 193-6. Retrieved from http://www.ncbi.nlm.nih.gov/pubmed/2014414

Halkett, G. K. B., \& Kristjanson, L. J. (2007). Patients' perspectives on the role of radiation therapists. Patient Education and Counseling, 69(1-3), 76-83. http://doi.org/10.1016/j.pec.2007.07.004

Halkett, G. K. B., Schofield, P., O’Connor, M., York, D., Jefford, M., Jiwa, M., ... Aranda, S. (2012). Development and pilot testing of a radiation therapist-led educational intervention for breast cancer patients prior to commencing radiotherapy. Asia-Pacific Journal of Clinical Oncology, 8(3), e1-8. http://doi.org/10.1111/j.1743-7563.2012.01520.x

Halkett, G. K. B., Short, M., \& Kristjanson, L. J. (2009). How do radiation oncology health professionals inform breast cancer patients about the medical and technical aspects of their treatment? Radiotherapy and Oncology: Journal of the European Society for Therapeutic Radiology and Oncology, 90(1), 153-9. http://doi.org/10.1016/j.radonc.2008.05.017

Hamarneh, A. (2015). Lack of language skills and knowledge of local culture in international medical graduates: Implications for the NHS. Hospital Practice 
4094 4095 4096 4097

4098 4099 4100 4101

4102 4103 4104 4105

4106 4107 4108 4109

(1995), 43(4), 208-11. Retrieved from http://www.ncbi.nlm.nih.gov/pubmed/26234814

Hamilton, C., Adolphs, S., \& Nerlich, B. (2007). The meanings of "risk": A view from corpus linguistics. Discourse and Society, 18(2), 163-181. http://doi.org/10.1177/0957926507073374

Hamilton, H. E., \& Chou, W. S. (2010). The Routledge Handbook of Language and Health Communication. Routledge. http://doi.org/10.4324/9781315856971

Hamilton, J., \& Woodward-Kron, R. (2010). Developing cultural awareness and intercultural communication through multimedia: A case study from medicine and the health sciences. System, 38(4), 560-568.

http://doi.org/10.1016/j.system.2010.09.015

Hamrosi, K. K., Aslani, P., \& Raynor, D. K. (2014). Beyond needs and expectations: Identifying the barriers and facilitators to written medicine information provision and use in Australia. Health Expectations, 17(2). http://doi.org/10.1111/j.13697625.2011.00753.x

Hamrosi, K. K., Raynor, D. K., \& Aslani, P. (2013). Pharmacist and general practitioner ambivalence about providing written medicine information to patients-A qualitative study. Research in Social and Administrative Pharmacy, 9(5). http://doi.org/10.1016/j.sapharm.2013.02.006

Hamrosi, K. K., Raynor, D. K., \& Aslani, P. (2014). Pharmacist, general practitioner and consumer use of written medicine information in Australia: Are they on the same page? Research in Social and Administrative Pharmacy, 10(4). http://doi.org/10.1016/j.sapharm.2013.10.002

Hamrosi, K. K., Raynor, D. K., \& Aslani, P. (2014). Enhancing provision of written medicine information in Australia: pharmacist, general practitioner and consumer perceptions of the barriers and facilitators. BMC Health Services Research, 14. http://doi.org/10.1186/1472-6963-14-183

Hancock, J. T., Woodworth, M. T., \& Porter, S. (2013). Hungry like the wolf: A word-pattern analysis of the language of psychopaths. Legal and Criminological Psychology, 18(1), 102-114. http://doi.org/10.1111/j.2044-8333.2011.02025.x

Hansberry, D. R., John, A., John, E., Agarwal, N., Gonzales, S. F., \& Baker, S. R. (2014). A Critical Review of the Readability of Online Patient Education Resources From RadiologyInfo.Org. American Journal of Roentgenology, 202(3), 566-575. http://doi.org/10.2214/AJR.13.11223

Hansberry, D. R., Ramchand, T., Patel, S., Kraus, C., Jung, J., Agarwal, N., ... Baker, S. R. (2014). Are we failing to communicate? Internet-based patient education materials and radiation safety. European Journal of Radiology, 83(9), 1698 1702. http://doi.org/10.1016/j.ejrad.2014.04.013 
Harvey, K., \& Brown, B. (2012). Health Communication and Psychological Distress: Exploring the Language of Self-harm. Canadian Modern Language Review. http://doi.org/10.3138/cmlr.1103

Harvey, K., Churchill, D., Crawford, P., Brown, B., Mullany, L., Macfarlane, A., ... Health, M. A. (2008). Health communication and adolescents: what do their emails tell us? Family Practice, 25(4), 304-311. http://doi.org/10.1093/fampra/cmn029

Harvey, K., Locher, M. A., \& Mullany, L. (2013). “Can I Be at Risk of Getting AIDS?" A Linguistic Analysis of Two Internet Columns on Sexual Health *. Linguistik Online, 59(2/13), 111-132. Retrieved from https://edoc.unibas.ch/28087/1/20131011103809_5257b8f1255f6.pdf

Haw, C., \& Stubbs, J. (2011). Patient information leaflets for antidepressants: Are patients getting the information they need? Journal of Affective Disorders, 128(1-2), 165-170. Retrieved from https://www.sciencedirect.com/science/article/pii/S0165032710004623

Hayes, E., Dua, R., Yeung, E., \& Fan, K. (2018). Patient understanding of commonly used oral medicine terminology. British Dental Journal, 223(11), 842. http://doi.org/10.1038/sj.bdj.2017.991

HAYES, S. C., \& FARNILL, D. (1993). Medical training and English language proficiency. Medical Education, 27(1), 6-14. http://doi.org/10.1111/j.13652923.1993.tb00222.x

Henderson, D., McGrath, P. D., \& Patton, M. A. (2017). Experience of clinical supervisors of international medical graduates in an Australian district hospital. Australian Health Review : A Publication of the Australian Hospital Association, 41(4), 365-371. http://doi.org/10.1071/AH15094

Herber, O. R., Gies, V., Schwappach, D., Thürmann, P., \& Wilm, S. (2014). Patient information leaflets: informing or frightening? A focus group study exploring patients' emotional reactions and subsequent behavior towards package leaflets of commonly prescribed medications in family practices. BMC Family Practice, 15(1). http://doi.org/10.1186/1471-2296-15-163

Herfs, P. G. P. (2014). Aspects of medical migration with particular reference to the United Kingdom and the Netherlands. Human Resources for Health, 12(1), 59. http://doi.org/10.1186/1478-4491-12-59

Heritage, J., \& Sefi, S. (1992). Dilemmas of advice: Aspects of the delivery and reception of advice in interactions between healh visitors and first-time mothers. Talk at Work: Interaction in Institutional Settings. Retrieved from http://www.sscnet.ucla.edu/soc/faculty/heritage/Site/Publications_files/DILEMM AS_OF_ADVICE.pdf 
Hewett, D. G., Watson, B. M., \& Gallois, C. (2015). Communication between hospital doctors: Underaccommodation and interpretability. Language \& Communication, 41, 71-83. http://doi.org/10.1016/j.langcom.2014.10.007

Hibbard, J. H., Peters, E., Dixon, A., \& Tusler, M. (2007). Consumer competencies and the use of comparative quality information: It isn't just about literacy. Medical Care Research and Review, 64(4), 379-394. http://doi.org/10.1177/1077558707301630

Hibbard, J. H., Peters, E., Dixon, A., \& Tusler, M. (2007). Consumer Competencies and the Use of Comparative Quality Information. Medical Care Research and Review, 64(4), 379-394. http://doi.org/10.1177/1077558707301630

Hinds, C., Streater, A., \& Mood, D. (1995). Functions and preferred methods of receiving information related to radiotherapy. Perceptions of patients with cancer. Cancer Nursing, 18(5), 374-84. Retrieved from http://www.ncbi.nlm.nih.gov/pubmed/7585492

Hinkel, E. (1995). The Use of Modal Verbs as a Reflection of Cultural Values. TESOL Quarterly, 29(2), 325-343. http://doi.org/10.2307/3587627

Hirsh, D., Clerehan, R., Staples, M., Osborne, R. H., \& Buchbinder, R. (2009). Patient assessment of medication information leaflets and validation of the Evaluative Linguistic Framework (ELF). Patient Education and Counseling, 77(2), 248254. http://doi.org/10.1016/j.pec.2009.03.011

Hoekje, B. J. (2007). Medical discourse and ESP courses for international medical graduates (IMGs). English for Specific Purposes, 26(3), 327-343. http://doi.org/10.1016/j.esp.2006.09.002

Hoffmann, K., Ristl, R., Heschl, L., Stelzer, D., \& Maier, M. (2014). Antibiotics and their effects: what do patients know and what is their source of information? The European Journal of Public Health, 24(3), 502-507. http://doi.org/10.1093/eurpub/ckt112

Hoffmann, T. C., \& Del Mar, C. (2017). Clinicians' Expectations of the Benefits and Harms of Treatments, Screening, and Tests. JAMA Internal Medicine, 177(3), 407. http://doi.org/10.1001/jamainternmed.2016.8254

Hoffmann, T. C., Légaré, F., Simmons, M. B., McNamara, K., McCaffery, K., Trevena, L. J., ... Del Mar, C. B. (2014). Shared decision making: what do clinicians need to know and why should they bother? The Medical Journal of Australia, 201(1), 35-39. http://doi.org/10.5694/mja14.00002

Hoffmann, T., McKenna, K., Worrall, L., \& Read, S. J. (2007). Randomised trial of a computer-generated tailored written education package for patients following stroke. Age and Ageing, 36(3), 280-6. http://doi.org/10.1093/ageing/afm003 
Holmberg, O., Czarwinski, R., \& Mettler, F. (2010). The importance and unique aspects of radiation protection in medicine. European Journal of Radiology, 76(1), 6-10. http://doi.org/10.1016/j.ejrad.2010.06.031

Hsu, W. (2013). Bridging the vocabulary gap for EFL medical undergraduates: The establishment of a medical word list. Language Teaching Research, 17(4), 454484. http://doi.org/10.1177/1362168813494121

Hudson, R. (1994). About 37\% of Word-Tokens are Nouns. Language, 70(2), 331339. http://doi.org/10.2307/415831

Hunston, S. (2006). Corpus Linguistics. In Encyclopedia of Language \& Linguistics. http://doi.org/10.1016/B0-08-044854-2/00944-5

Hyden, L.-C., Mishler, E. G., Hydén, L., \& Mishler, E. G. (2003). LANGUAGE AND MEDICINE. Annual Review of Applied Linguistics, 19, 174-192. http://doi.org/10.1017/S0267190599190093

Hyland, K. (2015). Corpora and written academic English. In The Cambridge Handbook of English Corpus Linguistics (pp. 292-308). http://doi.org/10.1007/9781139764377.017

Hyland, K. (2002). Directives: Argument and engagement in academic writing. Applied Linguistics, 23(2), 215-239. http://doi.org/10.1093/applin/23.2.215

Hyland, K. (2008). As can be seen: Lexical bundles and disciplinary variation. English for Specific Purposes, 27(1), 4-21. http://doi.org/10.1016/j.esp.2007.06.001

Hyland, K. (2012). Bundles in Academic Discourse. Annual Review of Applied Linguistics, 3212, 150-169. http://doi.org/10.1017/S0267190512000037

Ilić, N., Auchlin, A., Hadengue, A., Wenger, A., \& Hurst, S. A. (2013). Informed Consent Forms in Oncology Research: Linguistic Tools Identify Recurrent Pitfalls. AJOB Primary Research, 4(4), 39-54. http://doi.org/10.1080/21507716.2013.788101

Isaacman, D. J., Purvis, K., Gyuro, J., Anderson, Y., \& Smith, D. (1992). Standardized instructions: do they improve communication of discharge information from the emergency department? Pediatrics, 89(6 Pt 2), 1204-8. Retrieved from http://www.ncbi.nlm.nih.gov/pubmed/1594378

Jalali, Z. S., \& Moini, M. R. (2014). Structure of Lexical Bundles in Introduction Section of Medical Research Articles. Procedia - Social and Behavioral Sciences, 98, 719-726. http://doi.org/10.1016/j.sbspro.2014.03.473

Jalali, Z. S., Moini, M. R., \& Arani, M. A. (2015). Structural and Functional Analysis of Lexical Bundles in Medical Research Articles: A Corpus-Based Study. International Journal of Information Science and Management, 13(1), 51-69. Retrieved from http://ijism.ricest.ac.ir/index.php/ijism/article/viewFile/503/237 
Jensen, J. D., King, A. J., Carcioppolo, N., \& Davis, L. (2012). Why are Tailored Messages More Effective? A Multiple Mediation Analysis of a Breast Cancer Screening Intervention. The Journal of Communication, 62(5), 851-868. http://doi.org/10.1111/j.1460-2466.2012.01668.x

Jin, J., Sklar, G. E., Min Sen Oh, V., \& Chuen Li, S. (2008). Factors affecting therapeutic compliance: A review from the patient's perspective. Therapeutics and Clinical Risk Management, 4(1), 269-86. Retrieved from http://www.ncbi.nlm.nih.gov/pubmed/18728716

Johansson, S. (2010). Modals and semi-modals of obligation in American English: Some aspects of developments from 1990 until the present day. The Verb Phrase in English: Investigating Recent Language Change with Corpora, 372-380. http://doi.org/10.1017/CBO9781139060998.016

Johnson, A., \& Sandford, J. (2005). Written and verbal information versus verbal information only for patients being discharged from acute hospital settings to home: systematic review. Health Education Research, 20(4), 423-9. http://doi.org/10.1093/her/cyg141

Jones S. (2016). The Noncompliant vs The Non-adherent Patient. Retrieved November 24, 2018, from https://www.capphysicians.com/articles/noncompliantvs-non-adherent-patient

Juarez, G. (2011). Common english/spanish terminology use in radiology. Journal of Radiology Nursing, 30(1), 9-14. http://doi.org/10.1016/j.jradnu.2010.12.001

Jung, M., Ramanadhan, S., \& Viswanath, K. (2013). Effect of information seeking and avoidance behavior on self-rated health status among cancer survivors. Patient Education and Counseling, 92(1), 100-106. http://doi.org/10.1016/j.pec.2013.02.008

Kalra, G., Bhugra, D. K., \& Shah, N. (2012). Identifying and addressing stresses in international medical graduates. Academic Psychiatry: The Journal of the American Association of Directors of Psychiatric Residency Training and the Association for Academic Psychiatry, 36(4), 323-9. http://doi.org/10.1176/appi.ap.11040085

Kampa, R. J., Pang, J., \& Gleeson, R. (2006). Broken bones and fractures - an audit of patients' perceptions. Annals of the Royal College of Surgeons of England, 88(7), 663-6. http://doi.org/10.1308/003588406X149192

Kandula, S., \& Zeng-Treitler, Q. (2008). Creating a gold standard for the readability measurement of health texts. AMIA ... Annual Symposium Proceedings. AMIA Symposium, 2008, 353-7. Retrieved from http://www.ncbi.nlm.nih.gov/pubmed/18999150

Kazemi, M., Kohandani, M., \& Farzaneh, N. (2014). The Impact of Lexical Bundles on How Applied Linguistics Articles are Evaluated. Procedia - Social and 
Behavioral Sciences, 98, 870-875. http://doi.org/10.1016/J.SBSPRO.2014.03.494

Kecskes, I., \& Kirner-Ludwig, M. (2017). "It Would Never Happen in My Country I Must Say": A Corpus-Pragmatic Study on Asian English Learners' Preferred Uses of Must and Should. Corpus Pragmatics, 1(2), 91-134. http://doi.org/10.1007/s41701-017-0007-x

Kempe, C. B., Sullivan, K. A., \& Edmed, S. L. (2013). CE the effect of varying diagnostic terminology within patient discharge information on expected mild traumatic brain injury outcome. Clinical Neuropsychologist, 27(5), 762-778. http://doi.org/10.1080/13854046.2013.795245

Kenny, T. (1998). A PIL for every ill? Patient information leaflets (PILs): a review of past, present and future use. Family Practice, 15(5), 471-479. http://doi.org/10.1093/fampra/15.5.471

Kershaw, A. (2003). Patient use of the Internet to obtain health information. Nurs Times, 99(36), 30-32. Retrieved from http://www.ncbi.nlm.nih.gov/pubmed/14533432

Khan, J., \& Laxmi, S. (2013). Does the cancer patient want to know? Results from a study in an Indian tertiary cancer center. South Asian Journal of Cancer, 2(2), 57. http://doi.org/10.4103/2278-330X.110487

Kilgarriff, A. (2005). Language is never, ever, ever, random. Corpus Linguistics and Linguistic Theory, 1(2), 263-275. http://doi.org/10.1515/cllt.2005.1.2.263

King Edward's Hospital Fund for London. (1962). Information booklets for patients : report of an enquiry by the Division of Hospital Facilities. Retrieved from https://archive.kingsfund.org.uk/concern/published_works/

Knapp, P., Gardner, P. H., \& Woolf, E. (2016). Combined verbal and numerical expressions increase perceived risk of medicine side-effects: A randomized controlled trial of EMA recommendations. Health Expectations, 19(2), 264-274. http://doi.org/10.1111/hex.12344

Knight, D., Walsh, S., \& Papagiannidis, S. (2017). I'm having a spring clear out: A corpus-based analysis of e-transactional discourse. Applied Linguistics, 38(2), 235-257. http://doi.org/10.1093/applin/amv019

Kobayashi, L. C., Wardle, J., \& von Wagner, C. (2014). Limited health literacy is a barrier to colorectal cancer screening in England: Evidence from the English Longitudinal Study of Ageing. Preventive Medicine, 61, 100-105. http://doi.org/10.1016/j.ypmed.2013.11.012

Koch-Weser, S., DeJong, W., Rudd, R. E., Koch-Weser, S., DeJong, W., \& Rudd, R. E. (2009). Medical word use in clinical encounters. Health Expectations : An 
Koch-Weser, S., Rudd, R. E., \& Dejong, W. (2010). Quantifying Word Use to Study Health Literacy in Doctor-Patient Communication. J Health Commun., 15(6), 590-602.

Kopaczyk, J. (2013). Formulaic discourse across Early Modern English medical genres. Meaning in the History of English: Words and Texts in Context, 148, 257-300. http://doi.org/10.1075/slcs.148.12kop

Koteyko, N., Nerlich, B., Crawford, P., \& Wright, N. (2008). "Not rocket science" or "no silver bullet"? Media and government discourses about MRSA and cleanliness. Applied Linguistics, 29(2), 223-243. http://doi.org/10.1093/applin/amn006

Koteyko, N., Nerlich, B., Crawford, P., \& Wright, N. (2008). "Not rocket science" or "No silver bullet"? Media and Government Discourses about MRSA and Cleanliness. Applied Linguistics, 292, 223-243. http://doi.org/10.1093/applin/amn006

Krankl, J. T., Shaykevich, S., Lipsitz, S., \& Lehmann, L. S. (2011). Patient predictors of colposcopy comprehension of consent among English- and Spanish-speaking women. Women's Health Issues : Official Publication of the Jacobs Institute of Women's Health, 21(1), 80-5. http://doi.org/10.1016/j.whi.2010.07.009

Lampert, A., Wien, K., Haefeli, W. E., \& Seidling, H. M. (2016). Guidance on how to achieve comprehensible patient information leaflets in four steps. International Journal for Quality in Health Care, 28(5), 634-638. http://doi.org/10.1093/intqhc/mzw077

Landmark, A. M. D., Gulbrandsen, P., \& Svennevig, J. (2015). Whose decision? Negotiating epistemic and deontic rights in medical treatment decisions. Journal of Pragmatics, 78, 54-69. http://doi.org/10.1016/j.pragma.2014.11.007

Landmark, A. M. D., Svennevig, J., Gerwing, J., \& Gulbrandsen, P. (2017). Patient involvement and language barriers: Problems of agreement or understanding? Patient Education and Counseling, 100(6), 1092-1102. http://doi.org/10.1016/j.pec.2016.12.006

Lawrence, H., Poggenpoel, M., \& Myburgh, C. (2011). Experiences of being a therapy radiographer. Health SA Gesondheid, 16(1), 7 pages. http://doi.org/10.4102/hsag.v16i1.596

Lee, D. Y. (2001). Genres, Registers, Text Types, Domains, and Styles: Clarifying the Concepts and Navigating a Path Through the Bnc Jungle. http://doi.org/10.1016/S1364-6613(00)01594-1 
Leech, G. (1971). \{M\}eaning and the \{English\} \{V\}erb. Retrieved from https://books.google.ch/books?id=ISisAgAAQBAJ\&source=gbs_slider_cls_meta data_1_mylibrary

Leech, G. (2004). A new Gray's Anatomy of English grammar. English Language and Linguistics, 8(1), 121-147. http://doi.org/10.1017/S1360674304001273

Leech, G. (2004). Developing Linguistic Corpora: a Guide to Good Practice: Metadata for corpus work. Retrieved from https://ota.ox.ac.uk/documents/creating/dlc/chapter1.htm

Legato, M. J., Johnson, P. A., \& Manson, J. E. (2016). Consideration of Sex Differences in Medicine to Improve Health Care and Patient Outcomes. JAMA, 316(18), 1865. http://doi.org/10.1001/jama.2016.13995

Lei, L., \& Liu, D. (2016). A new medical academic word list: A corpus-based study with enhanced methodology. Journal of English for Academic Purposes, 22, 4253. http://doi.org/10.1016/J.JEAP.2016.01.008

León-Araúz, P., \& Reimerink, A. (2015). ScienceDirect Signs and symptoms in the psychiatric domain: a corpus analysis. Procedia - Social and Behavioral Sciences, 173, 285-292. http://doi.org/10.1016/j.sbspro.2015.02.067

Lerner, E. B., Jehle, D. V, Janicke, D. M., \& Moscati, R. M. (2000). Medical communication: do our patients understand? The American Journal of Emergency Medicine, 18(7), 764-6. http://doi.org/10.1053/ajem.2000.18040

Levin, M. E. (2006). Different use of medical terminology and culture-specific models of disease affecting communication between Xhosa-speaking patients and English-speaking doctors at a South African paediatric teaching hospital. South African Medical Journal, 96(10), 1080-1084.

Lewis, M. A., \& Newton, J. T. (2006). An evaluation of the quality of commercially produced patient information leaflets. British Dental Journal, 201(2), 114-7; discussion 100. http://doi.org/10.1038/sj.bdj.4813846

Leydon, G. M., Boulton, M., Moynihan, C., Jones, A., Mossman, J., Boudioni, M., \& McPherson, K. (2000). Cancer patients' information needs and information seeking behaviour: in depth interview study. BMJ (Clinical Research Ed.), 320(7239), 909-13. Retrieved from http://www.ncbi.nlm.nih.gov/pubmed/10742000

Lindström, A., \& Weatherall, A. (2015). Orientations to epistemics and deontics in treatment discussions. Journal of Pragmatics, 78, 39-53. http://doi.org/10.1016/j.pragma.2015.01.005

Little, P., Dorward, M., Warner, G., Moore, M., Stephens, K., Senior, J., \& Kendrick, T. (2004). Randomised controlled trial of effect of leaflets to empower patients in 
consultations in primary care. BMJ (Clinical Research Ed.), 328(7437), 441. http://doi.org/10.1136/bmj.37999.716157.44

Lövestam, E., Fjellström, C., Koochek, A., \& Andersson, A. (2014). The power of language on patient-centredness: linguistic devices in the dietetic notes of patient records. International Journal of Applied Linguistics, 25(2), n/a-n/a. http://doi.org/10.1111/ijal.12064

Lucas, P., Lenstrup, M., Prinz, J., Williamson, D., Yip, H., \& Tipoe, G. (1997). Language as a barrier to the acquisition of anatomical knowledge. Medical Education, 31(2), 81-86. http://doi.org/10.1111/j.1365-2923.1997.tb02463.x

Lucassen, E. B. (2015). Treatment of Multiple Sclerosis in Switzerland and the United States: What can be Learned from our Differences? Journal of Multiple Sclerosis, 02(04), 1-1. http://doi.org/10.4172/2376-0389.1000e107

Lustria, M. L. A., Cortese, J., Brown, L. L., Davis, R., Mahabi, V., Bae, B. J., \& Plotnikova, K. A. (2008). All computer-tailored online health interventions are not created equal. Proceedings of the American Society for Information Science and Technology, 44(1), 1-6. http://doi.org/10.1002/meet.1450440361

Lustria, M. L. A., Noar, S. M., Cortese, J., Van Stee, S. K., Glueckauf, R. L., \& Lee, J. (2013). A meta-analysis of web-delivered tailored health behavior change interventions. Journal of Health Communication, 18(9), 1039-1069. http://doi.org/10.1080/10810730.2013.768727

Lysanets, Y., Morokhovets, H., \& Bieliaieva, O. (2017). Stylistic features of case reports as a genre of medical discourse. Journal of Medical Case Reports, 11(1), 83. http://doi.org/10.1186/s13256-017-1247-x

Łyszczarz, B. (2017). Gender bias and sex-based differences in health care efficiency in Polish regions. International Journal for Equity in Health, 16(1), 8. http://doi.org/10.1186/s12939-016-0501-y

Maat, H. P., \& Klaassen, R. (1994). Side Effects of Side Effect Information in Drug Information Leaflets. Journal of Technical Writing and Communication, 24(4), 389-404. http://doi.org/10.2190/LEK9-VUJP-L3B9-V2LD

Maat, H. P., \& Lentz, L. (2011). Using sorting data to evaluate text structure: An evidence-based proposal for restructuring patient information leaflets. Technical Communication, 58(3), 197-216.

Macdonald, M. N. (2002). Pedagogy, pathology and ideology: The production, transmission and reproduction of medical discourse. Discourse and Society, 13(4), 447-467. http://doi.org/10.1177/0957926502013004453

Mack, J. E., Meltzer-Asscher, A., Barbieri, E., \& Thompson, C. K. (2013). Neural correlates of processing passive sentences. Brain Sciences, 3(3), 1198-214. http://doi.org/10.3390/brainsci3031198 
Maggs, F. M., Jubb, R. W., \& Kemm, J. R. (1996). Single-blind randomized controlled trial of an educational booklet for patients with chronic arthritis. British Journal of Rheumatology, 35(8), 775-7. Retrieved from http://www.ncbi.nlm.nih.gov/pubmed/8761192

Marco, M. J. L. (2000). Collocational frameworks in medical research papers: a genre-based study. English for Specific Purposes, 19(1), 63-86. http://doi.org/10.1016/S0889-4906(98)00013-1

Marshall, A. L., Leslie, E. R., Bauman, A. E., Marcus, B. H., \& Owen, N. (2003). Print versus website physical activity programs: a randomized trial. American Journal of Preventive Medicine, 25(2), 88-94. Retrieved from http://www.ncbi.nlm.nih.gov/pubmed/12880874

Martin, J. R. (2014). Evolving systemic functional linguistics: beyond the clause. Functional Linguistics, 1(1), 3. http://doi.org/10.1186/2196-419X-1-3

Martínez, A. C. L. (2002). Empirical examination of EFL readers' use of rhetorical information. English for Specific Purposes, 21(1), 81-98. https://doi.org/10.1016/s0889-4906(00)00029-6

Mathers, S. A., McKenzie, G. A., \& Robertson, E. M. (2011). A necessary evil: The experiences of men with prostate cancer undergoing imaging procedures. Radiography, 17(4), 284-291. http://doi.org/10.1016/J.RADI.2011.06.005

Mathers, S. a, Chesson, R. a, \& Mckenzie, G. A. (2009). The information needs of people attending for computed tomography (CT): what are they and how can they be met? Patient Education and Counseling, 77(2), 272-8. http://doi.org/10.1016/j.pec.2009.03.030

Matthiessen, C. (201AD). Applying systemic functional linguistics in healthcare contexts. Text\&Talk, 33(4-5)(437-467). Retrieved from https://www-degruytercom.openathens-proxy.swan.ac.uk/downloadpdf/j/text.2013.33.issue-4-5/text2013-0021/text-2013-0021.pdf

Mayberry Margaret, \& Mayberry John. (2002). Consent with understanding: a movement towards informed decisions. Clinical Medicine Royal College of Physicians Journal, 2, 523-6. Retrieved from www.bailii.org

Maynard, D. W., \& Heritage, J. (2005). Conversation analysis, doctor-patient interaction and medical communication. Medical Education, 39, 428-435. http://doi.org/10.1111/j.1365-2929.2005.02111.x

Mazzi, D. (2016). "It is natural for you to be afraid...": On the discourse of web-based communication with patients. Language Learning in Higher Education, 6(1). http://doi.org/10.1515/cercles-2016-0011

McCabe, M., Morgan, F., Curley, H., Begay, R., \& Gohdes, D. M. (2005). The informed consent process in a cross-cultural setting: is the process achieving the 

http://www.ncbi.nlm.nih.gov/pubmed/15825977

McCartney, M. (2013). Patient information leaflets: "a stupid system”. BMJ (Clinical Research Ed.), 347, f4748. http://doi.org/10.1136/bmj.f4748

McEnery, A., Xiao, Z., \& Tono, Y. (2006). Corpus-based Language Studies: An Advanced Resource Book. Corpus-based Language Studies: An Advanced Resource Book ((Routledge, Vol. 86). London: London: Routledge. http://doi.org/10.1016/j.jvir.2007.12.450

McEnery, T., \& Hardie, A. (2011). Corpus linguistics: Method, theory and practice. Corpus Linguistics: Method, Theory and Practice. Cambridge: Cambridge University Press. http://doi.org/10.1017/CBO9780511981395

McEnteggart, G. E., Naeem, M., Skierkowski, D., Baird, G. L., Ahn, S. H., \& Soares, of Vascular and Interventional Radiology, 26(8), 1164-1168.

McGrath, P., Henderson, D., \& Holewa, H. (2013). Language issues: an important professional practice dimension for Australian International Medical Graduates. Communication \& Medicine, 10(3), 191-200. Retrieved from http://www.ncbi.nlm.nih.gov/pubmed/25233557

McMullan, M. (2006). Patients using the Internet to obtain health information: How this affects the patient-health professional relationship. Patient Education and Counseling, 63(1-2), 24-28. http://doi.org/10.1016/j.pec.2005.10.006

Meade, C. D., \& Smith, C. F. (1991). Readability formulas: Cautions and criteria. Patient Education and Counseling, 17(2), 153-158. http://doi.org/10.1016/07383991(91)90017-Y

Meadows, L. M., Mrkonjic, L. A., O’Brien, M. D., \& Tink, W. (2007). The importance of communication in secondary fragility fracture treatment and prevention. Osteoporosis International : A Journal Established as Result of Cooperation between the European Foundation for Osteoporosis and the National Osteoporosis Foundation of the USA, 18(2), 159-66. http://doi.org/10.1007/s00198-006-0213-z

4495 Michalski, K., Farhan, N., Motschall, E., Vach, W., \& Boeker, M. (2017). Dealing 4496 with foreign cultural paradigms: A systematic review on intercultural challenges 
of international medical graduates. PloS One, 12(7), e0181330. http://doi.org/10.1371/journal.pone.0181330

Millar, N., \& Budgell, B. S. (2008). The language of public health - A corpus-based analysis. Journal of Public Health, 16(5), 369-374. http://doi.org/10.1007/s10389-008-0178-9

Minton, T. D. (2015). In Defense of the Passive Voice in Medical Writing. Keio J Med, 64(1), 1-10. http://doi.org/10.2302/kjm.2014-0009-RE

Mira, J. J., Orozco-Beltrán, D., Pérez-Jover, V., Martínez-Jimeno, L., Gil-Guillén, V. F., Carratala-Munuera, C., ... Asencio-Aznar, A. (2013). Physician patient communication failure facilitates medication errors in older polymedicated patients with multiple comorbidities. Family Practice, 30(1), 56-63. http://doi.org/10.1093/fampra/cms046

Moore, R. J., \& Hallenbeck, J. (2010). Narrative Empathy and How Dealing with Stories Helps: Creating a Space for Empathy in Culturally Diverse Care Settings. Journal of Pain and Symptom Management, 40(3), 471-476. http://doi.org/10.1016/j.jpainsymman.2010.03.013

Morony, S., Webster, A. C., Buchbinder, R., Kirkendall, S., McCaffery, K. J., \& Clerehan, R. (2018). A Linguistic Analysis of Health Literacy Demands of Chronic Kidney Disease Patient Education Materials. HLRP: Health Literacy Research and Practice, 2(1), e1-e14. http://doi.org/10.3928/2474830720171227-01

Moss, B., \& Roberts, C. (2005). Explanations, explanations, explanations: how do patients with limited English construct narrative accounts in multi-lingual, multiethnic settings, and how can GPs interpret them? Family Practice, 22(4), 412-8. http://doi.org/10.1093/fampra/cmi037

Mottram, D. R., \& Reed, C. (1997). Comparative evaluation of patient information leaflets by pharmacists, doctors and the general public. Journal of Clinical Pharmacy and Therapeutics, 22(2), 127-134.

Mullany, L., Smith, C., Harvey, K., \& Adolphs, S. (2015). Am i anorexic? Weight, eating and discourses of the body in online adolescent health communication. Communication and Medicine, 12(2-3), 211-223. http://doi.org/10.1558/cam.16692

Munsour, E. E., Awaisu, A., Hassali, M. A. A., Darwish, S., \& Abdoun, E. (2017). Readability and Comprehensibility of Patient Information Leaflets for Antidiabetic Medications in Qatar. Journal of Pharmacy Technology, 33(4), 128136. http://doi.org/10.1177/8755122517706978

Murgic, L., Hébert, P. C., Sovic, S., \& Pavlekovic, G. (2015). Paternalism and autonomy: views of patients and providers in a transitional (post-communist) 

country. BMC Medical Ethics, 16(1), 65. http://doi.org/10.1186/s12910-015$0059-\mathrm{z}$

Murphy, F. (2001). Understanding the humanistic interaction with medical imaging technology. Radiography, 7(3), 193-201. http://doi.org/10.1053/radi.2001.0328

Murray, D. E. (1988). The context of oral and written language: A framework for mode and medium switching. Language in Society, 17(3), 351-373. http://doi.org/10.1017/S004740450001294X

Myhill, J. (1997). Should and ought: the rise of individually oriented modality in American English. English Language and Linguistics, 1(01), 3-23. http://doi.org/10.1017/S1360674300000332

Myhill, J. (1995). Change and continuity in the functions of the American English modals. Linguistics, 33(2), 157-212. http://doi.org/10.1515/ling.1995.33.2.157

Nagel, K., Wizowski, L., Duckworth, J. A., Cassano, J., Hahn, S. A., \& Neal, M. (2008). Using plain language skills to create an educational brochure about sperm banking for adolescent and young adult males with cancer. Journal of Pediatric Oncology Nursing, 25(4), 220-226. http://doi.org/10.1177/1043454208319973

Nair, K., Dolovich, L., Cassels, A., McCormack, J., Levine, M., Gray, J., ... Burns, S. (2002). What patients want to know about their medications. Focus group study of patient and clinician perspectives. Canadian Family Physician Medecin de Famille Canadien, 48, 104-10. Retrieved from http://www.ncbi.nlm.nih.gov/pubmed/11852597

Narumol, J., Arunrot, P., \& Krska, J. (2015). Survey of patients' experiences and their certainty of suspected adverse drug reactions. International Journal of Clinical Pharmacy, 37(1), 168-174. http://doi.org/10.1007/s11096-014-0060-5

Nerlich, B., \& Halliday, C. (2007). Avian flu: The creation of expectations in the interplay between science and the media. Sociology of Health and Illness, 29(1), 46-65. http://doi.org/10.1111/j.1467-9566.2007.00517.x

Nesselhauf, N. (2004). Collocations in a Learner Corpus. John Benjamins Publishing (Vol. 14). Amsterdam: John Benjamins Publishing Company. http://doi.org/10.1017/S0272263107270068

NHS (2003) Toolkit for producing patient information. Retrieved from https://www.uea.ac.uk/documents/246046/0/Toolkit+for+producing+patient+info rmation.pdf

Nissen, T., \& Wynn, R. (2014). The history of the case report: a selective review. JRSM Open, 5(4), 205427041452341. http://doi.org/10.1177/2054270414523410 
4602

4603

4604

4605

4606

Nokkonen, S. (2014). The register variation of need to in Spoken British English. Neuphilologische Mitteilungen, 115(1), 63-94. http://doi.org/10.3366/cor.2010.0003

Nutbeam, D. (2000). Health literacy as a public health goal: a challenge for contemporary health education and communication strategies into the $21 \mathrm{st}$ century. Health Promotion International, 15(3), 259-267. http://doi.org/10.1093/heapro/15.3.259

Nuyts, J., Byloo, P., \& Diepeveen, J. (2010). On deontic modality, directivity, and mood: The case of Dutch mogen and moeten. Journal of Pragmatics, 42(1), 1634. http://doi.org/10.1016/j.pragma.2009.05.012

Nwogu, K. N. (1997). The medical research paper: Structure and functions. English for Specific Purposes, 16(2), 119-138. http://doi.org/10.1016/S08894906(97)85388-4

O’Connell, R. L., Hartridge-Lambert, S. K., Din, N., St John, E. R., Hitchins, C., \& Johnson, T. (2013). Patients' understanding of medical terminology used in the breast clinic. The Breast, 22(5), 836-838. http://doi.org/10.1016/J.BREAST.2013.02.019

Ogden, J., Boden, J., Caird, R., Chor, C., Flynn, M., Hunt, M., Khan, K., M., \& K., Swade, S., \& Thapar, V. (1999). (1999). You're Depressed, No I'm not: GPs' and patients' different models of depression. British Journal of General Practice, $49,123-124$.

O'Grady, C., Dahm, M. R., Roger, P., \& Yates, L. (2014). Trust, talk and the dictaphone: Tracing the discursive accomplishment of trust in a surgical consultation. Discourse and Society, 25(1), 65-83. http://doi.org/10.1177/0957926513496354

Ong, L. M., de Haes, J. C., Hoos, a M., \& Lammes, F. B. (1995). Doctor-patient communication: a review of the literature. Social Science \& Medicine (1982), 40(7), 903-18. Retrieved from http://www.ncbi.nlm.nih.gov/pubmed/18666041

Ownby, R. L. (2005). Influence of vocabulary and sentence complexity and passive voice on the readability of consumer-oriented mental health information on the Internet. AMIA ... Annual Symposium Proceedings. AMIA Symposium, 2005, 585-9. Retrieved from http://www.ncbi.nlm.nih.gov/pubmed/16779107

Pan, F., Reppen, R., \& Biber, D. (2016). Comparing patterns of L1 versus L2 English academic professionals: Lexical bundles in Telecommunications research journals. Journal of English for Academic Purposes, 21, 60-71. http://doi.org/10.1016/j.jeap.2015.11.003

Pandiya, A. (2010). Readability and comprehensibility of informed consent forms for clinical trials. Perspectives in Clinical Research, 1(3), 98-100. Retrieved from http://www.ncbi.nlm.nih.gov/pubmed/21814628 
Papautsky, E. L., \& Shalin, V. L. (2017). Strategies of Using Textual Information: Implications for Delivery of Health Information to Patients and Families. In Proceedings of the 2017 International Symposium on Human Factors and Ergonomics in Health Care (pp. 79-83). http://doi.org/10.1177/2327857917061018

Patha, P. (2006). This Is Very Important - University of Helsinki Research Portal University of Helsinki. In F. S.-M. Maurizio Gotti (Ed.), Advances in Medical Discourse Analysis: Oral and Written Contexts (pp. 357-381). Peter Lang. Retrieved from https://tuhat.helsinki.fi/portal/en/publications/this-is-veryimport(af8a1b4a-4ec7-4609-961e-f4f315a4ff35).html

Paul, F., Jones, M. C., Hendry, C., \& Adair, P. M. (2007). The quality of written information for parents regarding the management of a febrile convulsion: a randomized controlled trial. Journal of Clinical Nursing, 16(12), 2308-22. http://doi.org/10.1111/j.1365-2702.2007.02019.x

Peacock, M. (2014). Modals in the construction of research articles: A crossdisciplinary perspective. Iberica, (27), 143-164.

Peckham, T. J. (1994). Doctor, have I got a fracture or a break? Injury, 25(4), 221222. http://doi.org/10.1016/0020-1383(94)90065-5

Pennebaker, J. W., \& King, L. A. (1999). Linguistic styles: Language use as an individual difference. Journal of Personality and Social Psychology, 77(6), 1296-1312. http://doi.org/10.1037/0022-3514.77.6.1296

Pérez-sabater, C., Pena-Martinez, G., Turney, E., \& Monero-Fleta, B. (2008). A Spoken Genre Gets Written: Online Football Commentaries in English, French, and Spanish. Written Communication, 25(2), 235-261. http://doi.org/10.1177/0741088307313174

Perkins, M. R. (1982). The core meanings of the English modals. Journal of Linguistics, 18(02), 245. http://doi.org/10.1017/S0022226700013608

Phillips, S. P. (2005). International Journal for Equity in Health Defining and measuring gender: A social determinant of health whose time has come. International Journal for Equity in Health, 4(11). http://doi.org/10.1186/14759276-4

Pieterse, A. H., Jager, N. A., Smets, E. M. A., \& Henselmans, I. (2013). Lay understanding of common medical terminology in oncology. Psycho-Oncology, 22(5), 1186-1191. http://doi.org/10.1002/pon.3096

Pilnick, A., Hindmarsh, J., \& Gill, V. T. (2010). Communication in Healthcare Settings: Policy, Participation and New Technologies. Communication in Healthcare Settings: Policy, Participation and New Technologies. WileyBlackwell. 
Pires, C. M., \& Cavaco, A. M. (2014). Exploring the perspectives of potential consumers and healthcare professionals on the readability of a package insert: A case study of an over-the-counter medicine. European Journal of Clinical Pharmacology, 70(5). http://doi.org/10.1007/s00228-014-1645-6

Pires, C., Vigário, M., \& Cavaco, A. (2015). Readability of medicinal package leaflets: A systematic review. Revista de Saude Publica. http://doi.org/10.1590/S0034-8910.2015049005559

Prabhu, A. V., Hansberry, D. R., Agarwal, N., Clump, D. A., \& Heron, D. E. (2016). Radiation Oncology and Online Patient Education Materials: Deviating From $\mathrm{NIH}$ and AMA Recommendations. International Journal of Radiation Oncology Biology Physics, 96(3), 521-528. http://doi.org/10.1016/j.ijrobp.2016.06.2449

Protheroe, J., Estacio, E. V., \& Saidy-Khan, S. (2015). Patient information materials in general practices and promotion of health literacy: An observational study of their effectiveness. British Journal of General Practice, 65(632), e192-e197. https://doi.org/10.3399/bjgp15X684013

Raynor, D. K., Savage, I., Knapp, P., \& Henley, J. (2004). We are the experts: People with asthma talk about their medicine information needs. Patient Education and Counseling. http://doi.org/10.1016/S0738-3991(03)00126-5

Rayson, P. (2015). Computational tools and methods for corpus compilation and analysis. In The Cambridge Handbook of English Corpus Linguistics (pp. 3249). http://doi.org/10.1007/9781139764377.003

Regitz-Zagrosek, V. (2012). Sex and gender differences in health. Science \&amp; Society Series on Sex and Science. EMBO Reports, 13(7), 596-603. http://doi.org/10.1038/embor.2012.87

Research, G. V. (2018). What are the factors influencing ehealth market growth? Retrieved October 19, 2018, from www.Healthworkscollective.com

Rice, R. E. (2006, January). Influences, usage, and outcomes of Internet health information searching: Multivariate results from the Pew surveys. International Journal of Medical Informatics. http://doi.org/10.1016/j.ijmedinf.2005.07.032

Richard Watson Todd. (2017). An Opaque Engineering Word List. English for Specific Purposes, 45. Retrieved from https://ac.elscdn.com/S0889490616300862/1-s2.0-S0889490616300862main.pdf?_tid=dcd9ef3d-7547-4960-a39af970e3dcd976\&acdnat=1533633466_28c34cf5fe4608f02acca6ad9bd00933

Roberts, C., Moss, B., Wass, V., Sarangi, S., \& Jones, R. (2005). Misunderstandings: a qualitative study of primary care consultations in multilingual settings, and educational implications. Medical Education, 39(5), 465-75. http://doi.org/10.1111/j.1365-2929.2005.02121.x 
Roberts, C., \& Sarangi, S. (2003). Uptake of Discourse Research in Interprofessional Settings: Reporting from Medical Consultancy. Applied Linguistics, 24(3), 338359+421. http://doi.org/10.1093/applin/24.3.338

Roeland, E., Cain, J., Onderdonk, C., Kerr, K., Mitchell, W., \& Thornberry, K. (2014). When Open-Ended Questions Don't Work: The Role of Palliative Paternalism in Difficult Medical Decisions. Journal of Palliative Medicine, 17(4), 415-420. http://doi.org/10.1089/jpm.2013.0408

Rooks, R. N., Wiltshire, J. C., Elder, K., BeLue, R., \& Gary, L. C. (2012). Health information seeking and use outside of the medical encounter: Is it associated with race and ethnicity? Social Science and Medicine, 74(2), 176-184. http://doi.org/10.1016/j.socscimed.2011.09.040

Rosenkrantz, A. B., \& Flagg, E. R. (2015). Survey-Based Assessment of Patients' Understanding of Their Own Imaging Examinations. Journal of the American College of Radiology, 12(6), 549-555. http://doi.org/10.1016/J.JACR.2015.02.006

Røst, T. B., Huseth, O., \& Grimsmo, A. (2008). Lessons from Developing an Annotated Corpus of Patient Histories. Journal of Computer Science and Engineering, 2(2), 162-179.

Royal College of Emergency Medicine. (2016). The Royal College of Emergency Medicine Best Practice Guideline Management of Radiology Results in the Emergency Department. Retrieved from https://www.rcem.ac.uk/docs/College Guidelines/5q. Management of Radiology Results in the Emergency Department (February 2016).pdf

Rozmovits, L., \& Ziebland, S. (2004). What do patients with prostate or breast cancer want from an Internet site? A qualitative study of information needs. Patient Education and Counseling, 53(1), 57-64. http://doi.org/10.1016/S07383991(03)00116-2

Rutten, L. J. F., Squiers, L., \& Hesse, B. (2006). Cancer-related information seeking: Hints from the 2003 Health Information National Trends Survey (HINTS). Journal of Health Communication, 11(SUPPL. 1), 147-156. http://doi.org/10.1080/10810730600637574

Ryhänen, A. M., Johansson, K., Virtanen, H., Salo, S., Salanterä, S., \& Leino-Kilpi, H. (2009). Evaluation of written patient educational materials in the field of diagnostic imaging. Radiography, 15(2), e1-e5. http://doi.org/10.1016/j.radi.2008.04.006

Sakai, H., Katsumata, N., \& Takahashi, M. (2017). Providing written information increases patient satisfaction: a web-based questionnaire survey of Japanese cancer survivors. Japanese Journal of Clinical Oncology, 47(7), 611-617. http://doi.org/10.1093/jjco/hyx043 
Salager-meyer, F. (1983). The Lexis of Fundamental English:Classificatory Framework and Rhetorical Function ( A Statistical Approach). Reading in a Foreign Language.

Salager-Meyer, F. (1990). Discoursal fiaws in Medical English abstracts: A genre analysis per research- and text-type*. Text, 10(4), 365-384.

Salager-Meyer, F. (1994). Hedges and textual communicative function in medical English written discourse. English for Specific Purposes, 13(2), 149-170. http://doi.org/10.1016/0889-4906(94)90013-2

Salager-Meyer, F. (1992). A text-type and move analysis study of verb tense and modality distribution in medical English abstracts. English for Specific Purposes, 11(2), 93-113. http://doi.org/10.1016/S0889-4906(05)80002-X

Scantlebury, A., Booth, A., \& Hanley, B. (2017). Experiences, practices and barriers to accessing health information: A qualitative study. International Journal of Medical Informatics, 103, 103-108. http://doi.org/10.1016/j.ijmedinf.2017.04.018

Schiffrin, D., Tannen, D., \& Hamilton, H. E. (2008). The \{Handbook\} of \{Discourse\} \{Analysis\}.

Schulz, P. J., \& Nakamoto, K. (2013). Health literacy and patient empowerment in health communication: The importance of separating conjoined twins. Patient Education and Counseling. http://doi.org/10.1016/j.pec.2012.09.006

Scott, M. (1997). PC ANALYSIS OF KEY WORDS -AND KEY KEY WORDS. System, 25(2), 233-245. http://doi.org/10.1016/S0346-251X(97)00011-0

Scott, M., \& Tribble, C. (2006). Key words of individual texts Aboutness and style. John Benjamin.

Seale, C., Charteris-Black\#, J., \& Charteris-Black, J. (2008). The interaction of age and gender in illness narratives. Ageing and Society, 28(7), 1025-1045. http://doi.org/10.1017/S0144686X0800737X

Seale, C., Ziebland, S., \& Charteris-Black, J. (2006). Gender, cancer experience and internet use: A comparative keyword analysis of interviews and online cancer support groups. Social Science \& Medicine, 62(10), 2577-2590. http://doi.org/10.1016/j.socscimed.2005.11.016

Segal, J. Z. (2009). Internet Health and the 21st-Century Patient: A Rhetorical View. Written Communication, 26(4), 351-369. http://doi.org/10.1177/0741088309342362

Semino, E., Demjén, Z., Hardie, A., Payne, S., \& Rayson, P. (2017). Metaphor, Cancer and the End of Life: A Corpus-Based Study. Metaphor, Cancer and the 
End of Life: A Corpus-Based Study. Routledge. http://doi.org/10.4324/9781315629834

Semino, E., \& Demjén, Z. (2017). The Cancer Card: Metaphor, Intimacy, and Humor in Online Interactions about the Experience of Cancer. In B. Hampe (Ed.), Metaphor (pp. 181-199). Cambridge: Cambridge University Press. http://doi.org/10.1017/9781108182324.011

Shaw, J., \& Baker, M. (2004). "Expert patient"-dream or nightmare? Bmj, 328(7442), 723-724. http://doi.org/10.1136/bmj.328.7442.723

Shea-Budgell, M. A., Kostaras, X., Myhill, K. P., \& Hagen, N. A. (2014). Information needs and sources of information for patients during cancer follow-up. Current Oncology, 21(4), 165. http://doi.org/10.3747/co.21.1932

Sheard, C., \& Garrud, P. (2006). Evaluation of generic patient information: effects on health outcomes, knowledge and satisfaction. Patient Education and Counseling, 61(1), 43-7. http://doi.org/10.1016/j.pec.2005.02.004

Shergill, I., Bahl, K., Farjad, M., Phipps, C., \& Fowlis, G. (2010). Patient information leaflets for Transrectal Ultrasound guided prostate biopsy: Results of North Thames deanery survey. BMC Research Notes, 3(1), 27. http://doi.org/10.1186/1756-0500-3-27

Shim, H., Ailshire, J., Zelinski, E., \& Crimmins, E. (2018). The Health and Retirement Study: Analysis of Associations Between Use of the Internet for Health Information and Use of Health Services at Multiple Time Points. Journal of Medical Internet Research, 20(5), e200. http://doi.org/10.2196/jmir.8203

Short, C. E., James, E. L., Plotnikoff, R. C., \& Girgis, A. (2011). Efficacy of tailoredprint interventions to promote physical activity: a systematic review of randomised trials. International Journal of Behavioral Nutrition and Physical Activity, 8(1), 113. http://doi.org/10.1186/1479-5868-8-113

Silver, M. P. (2015). Patient Perspectives on Online Health Information and Communication With Doctors: A Qualitative Study of Patients 50 Years Old and Over. Journal of Medical Internet Research, 17(1), e19. http://doi.org/10.2196/jmir.3588

Sinclair, J., \& Carter, R. (2004). Trust the text: language, corpus and discourse. Routledge. Retrieved from https://www.routledge.com/Trust-the-Text-LanguageCorpus-and-Discourse/Sinclair-Carter/p/book/9780203594070

Singh, N., Mohacsy, A., Connell, D. A., \& Schneider, M. E. (2017). A snapshot of patients' awareness of radiation dose and risks associated with medical imaging examinations at an Australian radiology clinic. Radiography, 23(2), 94-102. http://doi.org/10.1016/J.RADI.2016.10.011 
Sinha, A., Porter, T., \& Wilson, A. (2018). The Use of Online Health Forums by Patients With Chronic Cough: Qualitative Study. Journal of Medical Internet Research, 20(1), e19. http://doi.org/10.2196/jmir.7975

Skelton, J. R., \& Hobbs, F. D. (1999). Descriptive study of cooperative language in primary care consultations by male and female doctors. BMJ (Clinical Research Ed.), 318(7183), 576-579. http://doi.org/10.1136/bmj.318.7183.576

Skelton, J. R., Kai, J., \& Loudon, R. F. (2008). Cross-cultural communication in medicine: questions for educators. Medical Education, 35(3), 257-261. http://doi.org/10.1111/j.1365-2923.2001.00873.x

Skelton, J. (1997). The representation of truth in academic medical writing. Applied Linguistics, 18(2), 121-140. http://doi.org/10.1093/applin/18.2.121

Skelton, J. R., Wearn, A. M., \& Hobbs, F. D. R. (2002). A concordance-based study of metaphoric expressions used by general practitioners and patients in consultation. The British Journal of General Practice : The Journal of the Royal College of General Practitioners, 52(475), 114-8. Retrieved from http://www.ncbi.nlm.nih.gov/pubmed/11887876

Skelton, J. R., Wearn, A. M., \& Hobbs, F. D. R. (2002). "I" and "we": a concordancing analysis of how doctors and patients use first person pronouns in primary care consultations. Family Practice, 19(5), 484-488. http://doi.org/10.1093/fampra/19.5.484

Skelton, J. R., \& Whetstone, J. (2012). English for Medical Purposes and Academic Medicine: looking for common ground. Ibérica, 24(24), 87-102. Retrieved from http://www.redalyc.org/articulo.oa?id=287024476008

Skelton, J. (2008). Language and clinical communication : this bright Babylon. Radcliffe Pub.

Skelton, J. R., \& Hobbs, F. D. R. (1999). Concordancing: Use of language-based research in medical communication. Lancet, 353(9147), 108-111. http://doi.org/10.1016/S0140-6736(98)02469-6

Skjeggestad, E., Gerwing, J., \& Gulbrandsen, P. (2017). Language barriers and professional identity: A qualitative interview study of newly employed international medical doctors and Norwegian colleagues. Patient Education and Counseling, 100(8), 1466-1472. http://doi.org/10.1016/j.pec.2017.03.007

Skjeggestad, E., Norvoll, R., Sandal, G. M., \& Gulbrandsen, P. (2017). How do international medical graduates and colleagues perceive and deal with difficulties in everyday collaboration? A qualitative study. Scandinavian Journal of Public Health, 45(4). http://doi.org/10.1177/1403494817698286

Slade, D., Scheeres, H., Manidis, M., Iedema, R., Dunston, R., Stein-Parbury, J., ... McGregor, J. (2008). Emergency communication: the discursive challenges 
facing emergency clinicians and patients in hospital emergency departments. Discourse \& Communication, 2(3), 271-298. http://doi.org/10.1177/1750481308091910

Sloane, C., Whitley, S. A., Anderson, C., \& Holmes, K. (2010). Clark's Pocket Handbook for Radiographers. CRC Press. Retrieved from https://books.google.com/books?id=6i7SBQAAQBAJ\&pgis=1

Smith, S., \& Duman, M. (2009, December). The state of consumer health information: An overview. Health Information and Libraries Journal. Wiley/Blackwell (10.1111). http://doi.org/10.1111/j.1471-1842.2009.00870.x

Squiers, L., Finney Rutten, L. J., Treiman, K., Bright, M. A., \& Hesse, B. (2005). Cancer patients' information needs across the cancer care continuum: Evidence from the Cancer Information Service. Journal of Health Communication. http://doi.org/10.1080/10810730500263620

Stevenson, F., McNulty, G., \& Leontowitsch, M. (2012). Negotiating roles in pharmacy practice: Interactions across linguistic and cultural barriers. Communication and Medicine, 9(1), 83-94. http://doi.org/10.1558/cam.v9i1.83

Sudore, R. L., \& Schillinger, D. (2009). Interventions to Improve Care for Patients with Limited Health Literacy. Journal of Clinical Outcomes Management : JCOM, 16(1), 20-29. Retrieved from http://www.pubmedcentral.nih.gov/articlerender.fcgi?artid=2799039\&tool=pmce ntrez\&rendertype $=$ abstract

Sustersic, M., Jeannet, E., Cozon-Rein, L., Maréchaux, F., Genty, C., Foote, A., ... Bosson, J. L. (2013). Impact of information leaflets on behavior of patients with gastroenteritis or tonsillitis: A cluster randomized trial in french primary care. Journal of General Internal Medicine, 28(1), 25-31. http://doi.org/10.1007/s11606-012-2164-8

Swee Heng, C., Kashiha, H., \& Tan, H. (2014). Lexical Bundles: Facilitating University “Talk” in Group Discussions. English Language Teaching, 7(4). http://doi.org/10.5539/elt.v7n4p1

Taavitsainen, I., \& Pahta, P. (2000). Conventions of Professional Writing The Medical Case Report in a Historical Perspective. JEngL (Vol. 28). Retrieved from http://journals.sagepub.com.openathensproxy.swan.ac.uk/doi/pdf/10.1177/00754240022004875

Tailor, A., \& Ogden, J. (2009). Avoiding the term 'obesity': An experimental study of the impact of doctors' language on patients' beliefs. Patient Education and Counseling, 76(2), 260-264. http://doi.org/10.1016/j.pec.2008.12.016

Tausczik, Y. R., \& Pennebaker, J. W. (2010). The psychological meaning of words: LIWC and computerized text analysis methods. Journal of Language and Social Psychology. http://doi.org/10.1177/0261927X09351676 
The Medicines and Healthcare products Regulatory Agency. (2012). BEST PRACTICE GUIDANCE ON PATIENT INFORMATION LEAFLETS EXPLANATORY MEMORANDUM. Retrieved from http://www.ema.europa.eu/docs/en_GB/document_library/Template_or_form/20 0

Tian, C., Champlin, S., Mackert, M., Lazard, A., \& Agrawal, D. (2014). Readability, suitability, and health content assessment of web-based patient education materials on colorectal cancer screening. Gastrointestinal Endoscopy, 80(2). http://doi.org/10.1016/j.gie.2014.01.034

Tognini-Bonelli, E. (2001). Corpus Linguistics at Work. Studies in corpus linguistics. Amsterdam: John Benjamins. http://doi.org/10.1017/CBO9781107415324.004

Tong, V., Raynor, D. K., \& Aslani, P. (2017). User testing as a method for identifying how consumers say they would act on information related to over-the-counter medicines. Research in Social and Administrative Pharmacy, 13(3), 476-484. http://doi.org/10.1016/j.sapharm.2016.06.001

Tong, V., Raynor, D. K., \& Aslani, P. (2018). Receipt and use of spoken and written over-the-counter medicine information: insights into Australian and UK consumers' experiences. International Journal of Pharmacy Practice, 26(2), 129-137. http://doi.org/10.1111/ijpp.12382

Tutty, L., \& O'Connor, G. (1999). Patient information leaflets: Some pertinent guidelines. Radiography, 5(1), 11-14. http://doi.org/10.1016/S10788174(99)90003-2

Ukkola, L., Oikarinen, H., Henner, A., Haapea, M., \& Tervonen, O. (2017). Patient information regarding medical radiation exposure is inadequate: Patients' experience in a university hospital. Radiography, 23(4), e114-e119. Retrieved from https://www.sciencedirect.com/science/article/pii/S1078817417300524

Van Berkel, J., \& Gerritsen, M. (2012). Patient Information Leaflets in Flanders and the Netherlands: Unnecessary Differences? In P. Heynderickx, S. Dieltjens, G. Jacobs, P.Gillaerts, \& E. De Groot (Eds.), The language factor in international business: New perspectives on research, teaching and practice (pp. 151-171). Bern: Peter Lang. Retrieved from https://marinelgerritsen.files.wordpress.com/2017/11/van-berkel-gerritsen2012.pdf

Van Rosse, F., De Bruijne, M., Suurmond, J., Essink-Bot, M.-L., \& Wagner, C. (2016). Language barriers and patient safety risks in hospital care. A mixed methods study. International Journal of Nursing Studies, 54, 45-53. http://doi.org/10.1016/j.ijnurstu.2015.03.012

Vassallo, T., Mizzi, A., Depasquale, R., Maher, M., \& Rainford, L. (2018, October). The development of patient information leaflets incorporating patient diversity considerations: Varicocele embolisation and fluoroscopy guided joint injection 
Vaughan, E., \& O'Keeffe, A. (2015). Corpus Analysis. In The International Encyclopedia of Language and Social Interaction (pp. 1-17). Hoboken, NJ, USA: John Wiley \& Sons, Inc. http://doi.org/10.1002/9781118611463.wbielsi168

Verma, A., Griffin, A., Dacre, J., \& Elder, A. (2016). Exploring cultural and linguistic influences on clinical communication skills: a qualitative study of International Medical Graduates. BMC Medical Education, 16(1), 162. http://doi.org/10.1186/s12909-016-0680-7

Vihla, M. (1999). Medical Writing: Modality in Focus Volume 28 of Language and Computers: Studies in Practical Linguistics (28th ed.). Rodopi.

Villafranca, A., Kereliuk, S., Hamlin, C., Johnson, A., \& Jacobsohn, E. (2017). The Appropriateness of Language Found in Research Consent Form Templates: A Computational Linguistic Analysis. PloS One, 12(2), e0169143. http://doi.org/10.1371/journal.pone.0169143

Visschers, V. H. M., Meertens, R. M., Passchier, W. W. F., \& de Vries, N. N. K. (2009). Probability Information in Risk Communication: A Review of the Research Literature. Risk Analysis, 29(2), 267-287. http://doi.org/10.1111/j.1539-6924.2008.01137.x

Vom, J., \& Williams, I. (2017). Justification of radiographic examinations: What are the key issues? Journal of Medical Radiation Sciences, 64(3), 212-219. http://doi.org/10.1002/jmrs.211

Vrana, S. R., Vrana, D. T., Penner, L. A., Eggly, S., Slatcher, R. B., \& Hagiwara, N. (2018). Latent Semantic Analysis: A new measure of patient-physician communication. Social Science \& Medicine, 198, 22-26. http://doi.org/10.1016/J.SOCSCIMED.2017.12.021

Waisman, Y., Siegal, N., Siegal, G., Amir, L., Cohen, H., \& Mimouni, M. (2005). Role of diagnosis-specific information sheets in parents' understanding of emergency department discharge instructions. European Journal of Emergency Medicine: Official Journal of the European Society for Emergency Medicine, 12(4), 159-62. Retrieved from http://www.ncbi.nlm.nih.gov/pubmed/16034260

Wang, L.-W. W., Miller, M. J., Schmitt, M. R., \& Wen, F. K. (2013). Assessing readability formula differences with written health information materials: Application, results, and recommendations. Research in Social and Administrative Pharmacy, 9(5), 503-516. http://doi.org/10.1016/j.sapharm.2012.05.009 
Ward, J. (2009). A basic engineering English word list for less proficient foundation engineering undergraduates. English for Specific Purposes, 28(3), 170-182. http://doi.org/10.1016/J.ESP.2009.04.001

Waring, M. E., McManus, D. D., Amante, D. J., Darling, C. E., \& Kiefe, C. I. (2018, June 30). Online health information seeking by adults hospitalized for acute coronary syndromes: Who looks for information, and who discusses it with healthcare providers? Patient Education and Counseling. Elsevier. http://doi.org/10.1016/j.pec.2018.06.016

Watson Todd, R. (2017). An opaque engineering word list: Which words should a teacher focus on? English for Specific Purposes, 45, 31-39. http://doi.org/10.1016/j.esp.2016.08.003

Webber, P. (2005). Interactive features in medical conference monologue. English for Specific Purposes, 24(2), 157-181. http://doi.org/10.1016/J.ESP.2004.02.003

Weidner, M. (2015). Telling somebody what to tell: "Prosze mi powiedzieć" in Polish doctor-patient interaction. Journal of Pragmatics, 78, 70-83. http://doi.org/10.1016/j.pragma.2015.01.006

Weingarten, S. R., Henning, J. M., Badamgarav, E., Knight, K., Hasselblad, V., Gano, A., \& Ofman, J. J. (2002). Interventions used in disease management programmes for patients with chronic illnesswhich ones work? Meta-analysis of published reports. BMJ, 325(7370), 925 . http://doi.org/10.1136/bmj.325.7370.925

Westerman, S., \& Wenger, N. K. (2016). Women and heart disease, the underrecognized burden: sex differences, biases, and unmet clinical and research challenges. Clinical Science, 130(8), 551-563. http://doi.org/10.1042/CS20150586

White, P., Smith, H., Webley, F., \& Frew, A. (2004). A survey of the quality of information leaflets on hayfever available from general practices and community pharmacies. Clinical and Experimental Allergy: Journal of the British Society for Allergy and Clinical Immunology, 34(9), 1438-43. http://doi.org/10.1111/j.1365-2222.2004.02055.x

Williams, I. A. (1999). Results Sections of Medical Research Articles: Analysis of Rhetorical Categories for Pedagogical Purposes. English for Specific Purposes (Vol. 18). Retrieved from https://ac.els-cdn.com/S0889490698000039/1-s2.0S0889490698000039-main.pdf?_tid=a4aedacb-b9b1-42ef-aa7900ce63eaa899\&acdnat=1540129496_a572425de1be180ae7ff22eeebbbab5f

Williams, N., \& Ogden, J. (2004). The impact of matching the patient's vocabulary: a randomized control trial. Family Practice, 21(6), 630-635. http://doi.org/10.1093/fampra/cmh610 
Williams, S. B., Huo, J., Dafashy, T. J., Ghaffary, C. K., Baillargeon, J. G., Morales, E. E., ... Kamat, A. M. (2017). Survival differences among patients with bladder cancer according to sex: Critical evaluation of radical cystectomy use and delay to treatment. Urologic Oncology: Seminars and Original Investigations, 35(10), 602.e1-602.e9. http://doi.org/10.1016/j.urolonc.2017.05.022

Winterbottom, A., Conner, M., Mooney, A., \& Bekker, H. L. (2007). Evaluating the quality of patient leaflets about renal replacement therapy across UK renal units. Nephrology, Dialysis, Transplantation: Official Publication of the European Dialysis and Transplant Association - European Renal Association, 22(8), 22916. http://doi.org/10.1093/ndt/gfm095

Wong, S. S. M., Bekker, H. L., Thornton, J. G., \& Gbolade, B. A. (2003). Choices about abortion method: assessing the quality of patient information leaflets in England and Wales. BJOG : An International Journal of Obstetrics and Gynaecology, 110(3), 263-6. Retrieved from http://www.ncbi.nlm.nih.gov/pubmed/12628264

Wright, P. (1999). Writing and information design of healthcare materials. In Christopher N Candlin and Ken Hyland (Ed.), Writing: Texts, Processes and Practices - Google Books (p. 330). Retrieved from https://books.google.ch/books?id=NWTJAwAAQBAJ\&pg=PA85\&lpg=PA85\&d $\mathrm{q}=$ patricia+wright,+writing+and+information+design+of+healthcare+materials \& source $=$ bl\&ots $=9$ p3SbVEfh\&sig=ZyeeNl10bGOZAuINCrg5mjUSovs\&hl=en\&sa=X\&ved=2ahUKEw i48biMgMXdAhWQgVwKHVPRCcAQ6AEwAnoECAgQ

Yagoda, B. (2006). You need to read this: How need to vanquished have to, must, and should. Slate Magazine. Retrieved from http://www.slate.com/articles/life/the_good_word/2006/07/you_need_to_read_thi s.html

Yang, A., Zheng, S., \& Ge, G. (2015). Epistemic modality in English-medium medical research articles: A systemic functional perspective. English for Specific Purposes, 38, 1-10. http://doi.org/10.1016/J.ESP.2014.10.005

Yang, M.-N. (2015). A nursing academic word list. English for Specific Purposes, 37, 27-38. http://doi.org/10.1016/j.esp.2014.05.003

Yates, L., Dahm, M. R., Roger, P., \& Cartmill, J. (2016). Developing rapport in interprofessional communication: Insights for international medical graduates. English for Specific Purposes, 42, 104-116. http://doi.org/10.1016/j.esp.2016.01.006

Zarcadoolas, C. (2011). The simplicity complex: Exploring simplified health messages in a complex world. Health Promotion International, 26(3), 338-350. http://doi.org/10.1093/heapro/daq075 
Zarcadoolas, C., Pleasant, A., \& Greer, D. S. (2005). Understanding health literacy: an expanded model. Health Promotion International, 20(2), 195-203. http://doi.org/10.1093/heapro/dah609

ZARCADOOLAS, C., PLEASANT, A., \& Greer, D. S. (2003). Elaborating a definition of health literacy: A commentary. Journal of Health Communication, 8(May), 119-120. http://doi.org/10.1080/713851982

Zarcadoolas, C., Sealy, Y., Levy, J., Dresser, M., Ponieman, D., Young, S. M., ... Silver, L. (2011). Health literacy at work to address overweight and obesity in adults: The development of the obesity action kit. Journal of Communication in Healthcare, 4(2), 88-101. http://doi.org/10.1179/175380611X13082296179316

ZHAO, J., \& WU, T. (2013). A genre analysis of medical abstracts by Chinese and English native speakers. Journal of Medical Colleges of PLA, 28(1), 60-64. http://doi.org/10.1016/S1000-1948(13)60018-0

Zheng, J., \& Yu, H. (2017). Readability Formulas and User Perceptions of Electronic Health Records Difficulty: A Corpus Study. Journal of Medical Internet Research, 19(3), e59. http://doi.org/10.2196/jmir.6962

Zummo Marianna Lye. (2016). Exploring web-mediated communication: A genrebased linguistic study for new patterns of doctor-patient interaction in online environment. Communication and Medicine, 12(2/3). http://doi.org/10.1558/cam.31897 